\title{
Students crossing cultural boundaries
}

within a transnational design course in China:

An activity theory analysis

By

Brenda Saris

\author{
A thesis \\ submitted to Victoria University of Wellington \\ in fulfilment of the requirements for the degree of \\ Doctor of Philosophy \\ Victoria University of Wellington
}

2020 


\section{Abstract}

\section{Students crossing cultural boundaries within a transnational design course in China: An activity theory analysis}

The focus of this thesis is on visual communication design (VCD) students' engagement with creative design process learning within a transnational context. The context is an international partnership between a higher education institution in New Zealand and Hunan City University (HNCU) in China. The Chinese government is currently positioned in a third wave of an internationalisation strategy which encourages cooperative agreements with foreign or overseas institutional partners situated within Chinese universities. For design institutions in particular, the Made in China government initiative has led universities to actively engage with design education approaches imported from the west. The aim for Chinese institutions is to encourage student creativity in order to build on government aspirations to move China from a manufacturing, to an innovation and design led economy.

Cultural historic activity theory (CHAT) was used to analyse data from a VCD studio classroom at HNCU in China. A three-level hierarchy of artefacts model was developed for the analysis, which by extention offers a CHAT approach for creative design scenarios. The study had two phases. The first comprised two project-based case studies exploring how creative design process learning occurred when the students were exposed to design thinking. Students were organised into dyads to foster collaborative work for the projects, a branding project, and a cultural project involving illustration design. Data gathered through video stimulated recall interviews with eight dyad participants (sixteen VCD students) and 200 written reflections were analysed. The second phase of the research focused on understanding the cultural and historic pedagogical context. Eight semi-structured interviews were conducted with local teachers at $\mathrm{HNCU}$, and observations were undertaken in Chinese language medium classrooms. 
Underpinning the findings, are the ways in which Chinese design education practices at $\mathrm{HNCU}$ are shaped by an interweaving of Confucian thought within contemporary social and political tenets (e.g., striving for perfection). The analysis revealed that familiar and unfamiliar learning practices, including previous models encountered by students in the classroom, together with an adjustment to new practices, directly impacted student actions. Imported educational practices resulted in tensions and contradictions between step-by-step and iterative design thinking processes, and collaboration within the division of labour. Non-creative and creative activity outcome conclusions were drawn, and it is argued that a fresh perspective emerged.

A creative craft practice situated within its historic and cultural context exists at HNCU. Key to the idea of creative craft practice is that historic and current sociocultural contexts participate in the creative process and contextual elements such as materiality, and teaching practices which use imitation, repetition and precedents, are assembled. The practice contained deeply intertwined student object-oriented motives of product over process, and productions of excellence or perfection. Over time, the efficacy of these motives, alongside drawing from examples for conceptual development, led to enhanced student agency and engagement. This overall finding challenges the creativity deficit belief about students from China, and the originality syndrome imposed on VCD students. The contribution is timely owing to a dearth of studies about graphic or VCD education in general and the potential influence of transnational teaching on creative design process learning in China. 


\section{Acknowledgements}

Many kindnesses were afforded to me through the duration of this research, some large and some small. Friends and colleagues at the higher education institution where I work were wonderful in the way they would quietly ask how it was going, offering support in a myriad of ways that they will never really know. In particular, I am thankful to Kaye Jujnovich who opened the door to this research opportunity; she believed that cross-cultural experiences were important for New Zealand teachers.

Three very special groups of people have played a huge part in this work, my supervisors, the staff and students at Hunan City University and my family. I was extremely fortunate and privileged to have had the wisdom and guidance of Dr Stephanie Doyle and Dr Judith Loveridge from the beginning to the end of the research. I cannot thank them enough for their constant support, encouragement and belief in me.

I am extremely grateful to Long, Xiangping, the Dean of Art and Design at Hunan City University who assisted in a multiple of ways. His enthusiasm for my research went above and beyond. Others at the university, including the teachers, students, participants, the interpreters, administration staff and newfound friends, thank you. Hunan City University and the city of Yiyang in China will forever hold a special place in my heart.

Finally, I must thank my family who accepted my mental absences and nearly four years of lost evenings, weekends and holidays. Paul, Milla and Neve were my anchors and my home. Jill Brandon, my high school art teacher and mother, set me off on this path many years ago, and my father, Dave, was always waving on the side-lines. The inspiration and support from Marcus and Alice Moore contributed much to this research. Alice was like a sister, she taught with me, travelled with me, read my work with me, and she laughed and cried with me. This was a rich chapter in our lives that may perhaps continue in ways yet unseen. 


\section{Table of contents}

\begin{tabular}{l|c}
\hline Abstract & III \\
\hline Acknowledgements & V \\
\hline Table of contents & VII \\
\hline List of figures & XII \\
\hline List of tables & XIII \\
\hline List appendices & XIV \\
\hline Acronym terms & XIV
\end{tabular}

$\begin{array}{ll}\text { Chapter 1. Introduction } & 1\end{array}$

1.1 Personal motivations for the research 1

The research site and transnational context 3

1.2 Internationalisation and transnational education 4

The significance of New Zealand international education policy 5

Opportunities and affordances of transnational higher education $\quad 5$

The significance of the 2025 'Made in China' political strategy 8

Pedagogical changes in China 99

$\begin{array}{ll}1.3 \text { A lens for examining VCD creative design process learning } & 10\end{array}$

$\begin{array}{ll}1.4 & \text { Artificial categories of east-west and north-south } \\ \end{array}$

$\begin{array}{llr}1.5 & \text { Research questions } & 11\end{array}$

1.6 Outline of the thesis and chapter introductions 12

$\begin{array}{ll}\text { Chapter 2. Literature Review } & 15\end{array}$

2.1 Graphic design or visual communication design? Three manifestos 16

$\begin{array}{ll}2.2 \text { Histories of design and design education } & 17\end{array}$

$\begin{array}{ll}\text { In the west } & 17\end{array}$

Origins of contemporary pedagogical practices $\quad 20$

Design thinking

Creativity $\quad 24$

$\begin{array}{ll}\text { In China } & 28\end{array}$ 
2.3 Culture and Confucianism

2.4 Empirical design and creativity research

Asian studies

Transnational higher education (TNHE) studies

2.5 Framing the research: The rationale for using cultural historic activity theory

3.1 The evolution of cultural historic activity theory (CHAT) 51

3.2 The CHAT generations $\quad 52$

3.3 Activity system (AS) components $\quad 59$

$\begin{array}{lll}3.4 & \text { Analytical framework } & 61\end{array}$

An AT methodological contribution for creative design process research 61

$\begin{array}{ll}\text { Analytical approach } & 65\end{array}$

$\begin{array}{ll}\text { Theory of creative activity } & 67\end{array}$

$\begin{array}{lll}3.5 & \text { Conclusion } & 68\end{array}$

4.1 Activity theory and case study

$\begin{array}{lll}4.2 & \text { Research design } & 73\end{array}$

Procedures for case studies: Phase one of the research $\quad 74$

The cultural and historic pedagogical context: Phase two of the research 76

$\begin{array}{lll}4.3 & \text { Data collection and purpose } & 77\end{array}$

$\begin{array}{ll}\text { Video stimulated recall interviews (VSRI) } & 78\end{array}$

Written reflections from the two-case studies 83

Teachers' semi-structured interviews

Observations in a Chinese medium (Mandarin) $\quad 84$ 
5.3 Overview and pedagogical underpinnings for two case studies

Theme one (1): Managing the effects of primary, secondary and tertiary artefact tensions

Theme two (2): Adjusting to collaborative learning approaches

Dyad 2: Lily and Connor

Theme three (3): Navigating imported design practices and processes

Dyad 4: Ricky and Simon 
$\begin{array}{llr}7.1 & \text { Analysis } & 164\end{array}$

Dyad 1: Will and Matt 164

Dyad 2: Aaron and Rebecca 169

Theme two (2A): Responding to teacher guidance 170

Theme four (4): Negotiating learning and family expectations 175

Dyad 3: Nina and Jasmine $\quad 180$

Dyad 4: Susan and Carl 184

$\begin{array}{llr}7.2 & \text { Discussion and conclusion } & 188\end{array}$

Chapter 8. Hunan City University (HNCU) teachers’ perspectives 195

$\begin{array}{llr}8.1 \text { Analysis } & 195\end{array}$

8.2 Theme one: Reconciling imitation with imagination, a tertiary artefact 197

8.3 Theme two: Encouraging cultural and social rules of perfection 207

$\begin{array}{ll}\text { Process, or product over process? } & 208\end{array}$

Quantity over quality $\quad 214$

$\begin{array}{ll}\text { Competitions } & 215\end{array}$

8.4 Theme 3: Balancing teaching roles with learning success in the division of labour 216

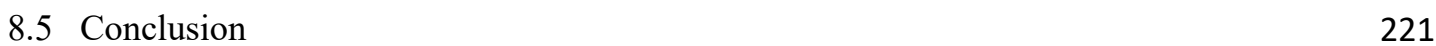

Chapter 9. Discussion

9.1 How do previous and current Chinese teaching practices shape

VCD creative design process learning?

Object-orientated motives in the production of product over process

Object-orientated motives in the production of excellence or perfection

and encouraging cultural and social rules of perfection

9.2 How do imported and unfamiliar educational practices influence

VCD learning activities?

Linear and iterative processes $\quad 235$

Collaborative practices in the division of labour 236

$\begin{array}{ll}\text { Copying and imitation } & 239\end{array}$ 
9.3 How do visual communication design (VCD) students at a university in China engage with creative design process learning?

9.4 Epilogue: Crossing cultural boundaries

Chapter 10. Conclusion

253

10.1 Research contributions

10.2 Limitations and possibilities for further research

259

References 


\section{List of figures}

Figure 3.1 (A). Vygotsky's model of a complex mediated act and $(B)$ the common reinterpretation of a mediated act

Figure $3.2(A)$. Second generation CHAT, from the basic structure of a human activity system, and $(B)$ production and consumption of an activity system

Figure 3.3. Third generation activity theory, two interacting systems as a minimal model 56

Figure 3.4. Analytical approach 66

$\begin{array}{lr}\text { Figure 4.1. Video captures at HNCU } & 81\end{array}$

Figure 4.2. Video capture of a student asleep at HNCU 82

$\begin{array}{lr}\text { Figure 5.1. Tea street in Yiyang } & 104\end{array}$

$\begin{array}{ll}\text { Figure 5.2. Freshmen military training at HNCU } & 109\end{array}$

$\begin{array}{ll}\text { Figure 5.3. Animation class at } \mathrm{HNCU} & 115\end{array}$

Figure 5.4. Exterior of a training school in the cluster of training schools, Changsha 117

$\begin{array}{ll}\text { Figure 5.5. Portrait drawing at the training school in Changsha } & 119\end{array}$

$\begin{array}{ll}\text { Figure 5.6. Still life, gestural reference material and student work } & 120\end{array}$

Figure 5.7. Student research instigated by Hua, and Shing's tea ceremony demonstration 122

Figure 6.1. Case study one: Pedagogical branding project. Four dyadic activity systems $\quad 127$

Figure 6.2. Amy's stages of design development referencing Chinese cultural elements 132

$\begin{array}{ll}\text { Figure 6.3. Dyad 1: Amy and John, themes and tensions } & 138\end{array}$

$\begin{array}{ll}\text { Figure 6.4. Evidence of copying } & 145\end{array}$

$\begin{array}{ll}\text { Figure 6.5. Dyad 2: Lily and Connor, themes and tensions } & 147\end{array}$

Figure 6.6. The dyadic process and Hana's subsequent computer development

$\begin{array}{ll}\text { of the concept } & 149\end{array}$

$\begin{array}{ll}\text { Figure 6.7. Dyad 3: Hana and Michael, themes and tensions } & 153\end{array}$

Figure 6.8. Dyad 4: Simon and Ricky, themes and tensions 157

Figure 6.9. Branding project: Key themes and findings from combined dyadic interactions, observations and reflections

$\begin{array}{ll}\text { Figure 7.1. Case study two: Miao playing card project. Four dyadic activity systems } & 163\end{array}$

$\begin{array}{ll}\text { Figure 7.2. Illustrator image trace technique } & 165\end{array}$

$\begin{array}{ll}\text { Figure 7.3. Dyad 1: Will and Matt, themes and tensions } & 169\end{array}$

Figure 7.4. Aarons new sketch, three examples of new references images, and his subsequent process in class 
Figure 7.6. Dyad 2: Aaron and Rebecca, themes and tensions

Figure 7.7. Dyad 3: Nina and Jasmine, themes and tensions

Figure 7.8. Dyad 4: Susan and Carl, theme and tensions

Figure 7.9. Miao cultural project: Key themes and findings from combined dyadic interactions, observations and reflections

Figure 8.1. Tension intersection

Figure 8.2: Student photoshop designs compiled from internet sourced images

Figure 8.3: Software skills learning, natural gas logo designs

Figure 8.4. Software skills learning, Bauhaus poster designs

Figure 8.5. Smog posters received from Lei

Figure 8.6. A Rubin vase

Figure 8.7. Completed student illustration

Figure 8.8. Analysis of teachers' interviews/data

Figure 9.1. Two interacting systems as a minimal model in third generation activity theory

Figure 9.2. A third-generation activity theory model to account for multiple voices

Figure 9.3. Student work in 2017, and the 2018 student mood-board

Figure 9.4. Iterative ideation sketches, and computer work using circles to develop the wing of a bird

Figure 9.5. Workbook explanations for the way colour and conceptual meanings for the solar terms were embedded into the illustration designs

\section{List of tables}

Table 3.1. A three-level hierarchy of artefacts

Table 4.1. Activity system analysis and a case study

Table 4.2. Research participants

Table 4.3. Case study participants

Table 4.4. Chinese teacher participants

Table 4.5. VSRI timeframes

Table 4.6. Video capture and interview time durations 
Table 4.7. Summary of data sources, participants and purpose

Table 4.8. Overall research design

Table 5.1. Timetabled morning hours (summer)

Table 5.2. Timetabled days and hours (summer)

Table 5.3. Data gathering phases and dates

Appendix table 1. Overview of twelve Branding project classes and video capture periods

Appendix table 2. Overview of twelve Miao illustration project classes and video capture periods

\section{List of appendices}

Appendix 4.1. Information sheet and consent form to video and interview students and teachers

Appendix 4.2. Translating confidentiality agreement

Appendix 4.3. Summary of participant ethical informed consents

Appendix 4.4. Examples of written reflections

\section{Acronym terms}

AS - Activity system/s

AT - Activity theory

$\mathrm{CHAT} \cdot \mathrm{Cultural}$ historic activity theory

DT $\cdot$ Design thinking

$\mathrm{HNCU} \cdot$ Hunan City University

TNHE - Transnational higher education

VCD $\cdot$ Visual communication design

VSRI • Video stimulated recall interview/s 


\section{Chapter 1. Introduction}

This research focused on visual communication design (VCD) students' engagement with creative design process learning at a single university site in China. Situated within a transnational three-plus-one agreement between a New Zealand higher education institution and Hunan City University (HNCU) in China, cultural historic activity theory (CHAT) was used to analyse contexts and creative design process learning in a HNCU classroom. By employing video stimulated recall, 16 student participants from two case study projects - a branding project and a cultural project involving illustration design - were interviewed and these were followed by eight semi-structured interviews with local teachers, and observations undertaken in Chinese medium classrooms. Uncovering answers to my research questions began with the way I became intrigued by students from China.

\subsection{Personal motivations for the research}

As a professional designer, turned visual communication design (VCD) educator, it was interesting to discover that it was actually quite difficult to teach a student how to enact a creative design process. For me, the transition from a novice to an expert was a gradual process which began twenty years before I entered a higher education classroom as a teacher (Cross, 2011). In this new classroom environment, I tried to remember what, and how, I had been taught at my school of design.

An early teaching experience that has remained with me is that of a student who was struggling to complete a poster design. There were a few intertwined problems which involved colour and hierarchy of scale between the design elements; the design elements were a headline, an illustration and a logo. I could not adequately explain how to improve the situation, so moved the design elements around on the computer screen to demonstrate the way in which colour and scale could be used to change the relationship between positive and negative space, thus improving the overall communication of the design. When I had finished talking, the look on the student's face, and the question he asked, stopped me in my tracks. "How did you do that?" 
I realised the answer lay in my twenty years of work experience, plus the five minutes I spent on that particular design. My job as a teacher was to work out how to help students tackle the difficult leap to synthesis (Ledewitz, 1985). Cross (2011) suggests that expert designers are different to other sorts of problem solvers (people who attempt to define the problem fully before making any attempts at a solution) because they have an ability to explore, frame and define a problem and a solution quickly and almost simultaneously. Over time, a characteristic of design expertise develops in which designers become solution-focused, not problem-focused. Therefore, the speed that I was used to working at, and the terminologies I used had to be adjusted (Logan, 2007). I now work in a more measured and reflective way (Schön, 1987), and have introduced aspects of design thinking into the classroom in the promotion of iterative ways of thinking and doing (Cross, 2011). Schön's (1987) ideas about relying on rough sketches to explain to students how various VCD elements (such as text, imagery or colour) can be composed by utilising the principles of design (for example, balance, rhythm or emphasis) to generate new meanings has been particularly useful.

At the end of 2015, I became involved in curriculum design and in teaching classes within a transnational partnership between a New Zealand higher education institution and Hunan City University (HNCU) in China. I had previously worked with students from China in New Zealand, and with my collegues had discussed the challenges these students seemed to have with creativity and critical thinking skills. It was not uncommon for those students to ask for direct guidence, and after I repeated what I expected them to do, I would sometimes find the students remained inactive, seemingly paralysed. It was also common to discover students copying work directly from the internet. When challenged, the students became visibly confused and upset. However, by the end of the academic year, most of the students would have settled into their studies, often producing surprising results. This was especially so when they focussed on illustration, or drew on familiar cultural aspects for their work, for example, Chinese zodiac animal signs or Chinese myths.

During my first teaching stint at HNCU, I encountered an enthusastic and vibrant VCD learning culture in which strong drawing skills appeared to play a role in the students' creative design process education at the university. One of the tasks assigned to the 
students involved scaling a small portrait photograph up onto a large sheet of paper by using a grid system. I found it difficult to understand why this technique, so familiar to me in a New Zealand classroom, seemed so difficult for the HNCU students. The interpreter I was working with in the classroom suggested that the students be allowed to draw their portraits onto the larger size paper without the grid. To my astonishment, they accomplished these drawings with great ease. This moment captivated me, ignited my curiosity about creative design learning in China, and prompted my research. I wanted to know more about how Chinese VCD students engage with creative design processes, and I wanted to address my unease with stereotypes about Chinese students and creativity.

\section{Designing learning curriculum for VCD students at Hunan City University}

When working with the VCD students at HNCU, it made sense that the reflective studio practice should draw on design thinking and creativity concepts (discussed in the literature review). However, in the classroom context, language and the unfamiliar cultural context became problematic. In a New Zealand classroom I could freely demonstrate design processes and talk about these, but I was not sure how I would foster similar creative design process learning in the HNCU students. I was entrusted to teach these students when I did not speak Mandarin. Although I had the benefit of an interpreter with me at all times, in the classroom I was an outsider when I was teaching and conducting the research at HNCU. At first I had little idea about creative design process learning at HNCU or how my VCD students might succeed within this transnational course. I also knew very little about the sociocultural influences on creative process learning. Undoubtedly, sources of confusion arose for the students, but I set out believing that I had as much to learn from the students as they did me.

\section{The research site and the transnational context}

The research took place at one university located in China. Hunan City University is based in the city of Yiyang, which is in the Hunan province. I spent a total of nine weeks at HNCU conducting the research, and during this time I had two roles, that of educator and that of a researcher. The study itself was situated within a transnational partnership between a New Zealand higher education institution and HNCU. 
The partnership aimed to prepare HNCU students for future study in New Zealand. A three-plus-one agreement means students spend the first three years studying in their home country institution before leaving to study for one year at an international partner institution. In this instance, two degrees were to be awarded on completion, one from each institution. The agreement between HNCU and the New Zealand institution is typical of these types of arrangements. Curriculum was designed and adapted for the Chinese context to scaffold learning at HNCU for VCD students before they leave China. In September of each year, over three years, a new intake of 100 students are taught an estimated $30 \%$ of their Chinese VCD degree content by New Zealand VCD staff at HNCU. Although course content was developed specifically for HNCU, it reflects some aspects of New Zealand diploma and degree programmes.

\subsection{Internationalisation and transnational education}

China, with one fifth of the world's population, is involved in many transnational initiatives such as the one this research was situated within. These initiatives tend to have their origins in high level government-led internationalisation strategies (NZ Government, 2018). In much the same way as companies use internationalisation as a way to expand quickly and gain access to global markets, higher education institutions internationalise to gain access to global education markets and establish relationships on a global level (Jain, Celo, \& Kumar, 2019). For a researcher working in this area, distinctions must be drawn between globalisation, which accounts for "world systems" and bears the marks of "dominant national cultures" in particular, American culture (Marginson, 2000, p. 24), and internationalisation which represents the specifics of a particular country, for example cultural and/or historic contexts (Yang, 2005). The concept of internationalisation within higher education has been defined as "the process of integrating an international, intercultural or global dimension into the purpose, functions or delivery of postsecondary education" (Knight, 2003, p. 2). The problem with this definition is that it represents imposed western experiences and perspectives (Yang, 2005). Yang argues for future research that does not adopt an implicit superiority of western approaches without first establishing validity of other strikingly different cultural contexts, values and heritage. He argues researchers should use indigenous knowledge which points to the importance of integrating one's own 
reflections on the local culture and/or society and/or history (Yang, 2005). This sentiment is echoed by others who argue for sensitivity towards culture in learning, to avoid tendencies to essentialise groups, and obscure the heterogeneity (diversity) of practice within both dominant and non-dominant groups (Nasir, Rosebery, Warren, \& Lee, 2005).

\section{The significance of New Zealand international education policy}

The New Zealand government's international education strategy 2018 - 2030 outlined three overarching goals: Excellent education and student experience, sustainable growth, and global citizens (NZ Government, 2018). The primary focus of the strategy was to grow the social and economic value of the country's fourth largest export sector by encouraging more international students to study in New Zealand. Projections of growth were ambitious based on global predictions that numbers of mobile international students would increase from five million in 2018 to seven or eight million by 2025, and New Zealand sought to increase its share of that market. However, numbers of students moving from China to New Zealand to study were projected to decline after 2025 owing to increased competition with other destination countries and increased capacity within the Chinese higher education system (NZ Government, 2018). Significantly for my research, within the $2018-2030$ strategy there are a number of medium to long-term key actions (from 2020 - 2030), one of which is to "explore opportunities to deliver education and education products offshore and online" (NZ Government, 2018, p. 17). This potentially signalled a policy interest in transnational initiatives to change the way New Zealand does business within offshore higher education sites.

\section{Opportunities and affordances of transnational higher education (TNHE)}

The projected drop in New Zealand-bound Chinese students has begun, and some agencies and providers seem locked into a one-way mobility model (Doyle, 2016); Doyle suggests a lack of policy recognition of "changing mobility flows" (p. 13). Similarly, Ryan (2016) highlighted the way international student flows are increasingly changing through research and academic collaborations, and transnational 
teaching partnerships and programmes. The numbers of students from China involved in transnational courses is significant (Montgomery, 2016). Although internal student mobility in China is on the rise, 544,500 students left China's shores to study through TNHE agreements in 2016 (Xu \& Montgomery, 2019). However, these numbers do not include the significant numbers of students who were taught in large classes in China at the beginning of a transnational programme and who decided to stay in China for their final year of study rather than travel overseas. My own experience attests to this. When teaching in China, through the rolling cohorts of 100 students entering the course each year, up to 300 students were taught twice yearly, yet only a handful made the decision to leave. Dai, Matthews, and Renshaw (2020) hint at the disproportionate number of TNHE students who choose to stay in China versus the number who choose to study abroad. These examples suggest that countries such as New Zealand may in the future be unable to rely on large numbers of international students coming to their shores. It is significant that locally driven change has been occurring within internationalisation in Asian countries for some time, and only a small number of New Zealand education providers are exploring opportunities for new initiatives and these are rarely more than "small scale cross-border joint initiatives" (Doyle, 2016, p. 15). The joint programme I was involved in and my research constitutes a small-scale initiative; however, the contribution is argued to be significant owing to the ways in which creative design process learning at a university in China has been contextualised and understood through empirical methods.

In TNHE, courses of study move across borders rather than students. Courses can move in different ways, including online distance learning, international branch campus location, and validated agreements, such as a three-plus-one partnership (Healey, 2015). Ryan (2016) identifies the four Ps of transnational higher education as "policies, programmes, pedagogy and partnerships" (p. 9). The benefit for all is that mutual understanding and learning are shared through the inherent cultural diversity that resides within movements of teachers and learners (Doyle, 2016). Interculturality, "encultured knowledge" and ultimately "cultural enrichment" are fostered when curiosity, interest and open-mindedness is experienced within TNHE exchange (Caruana \& Montgomery, 2015, p. 7). An emphasis on approaches that attend to local relevance, intercultural dimensions, customised practices and processes, delivery and 
goals is desirable (Knight, 2007; Yang, 2005). However, Madge and Noxolo (2015) caution against viewing student mobility in terms of student cultural capital and "social reproduction of class advantage" (p. 682). Such approaches require understanding and respect of local contexts whereby internationalisation complements and extends local dimensions in order to guard against homogenising tendencies.

\section{Risks of TNHE}

Since TNHE partnerships with China began at the beginning of the 1980s (Mok \& Han, 2016), there has been ample evidence of failed ventures, owing to issues associated with managing reputational risk, and imbalances between profit maximisation and maintaining academic quality (Healey, 2015). When Chinese institutions have difficulty in attracting students, they may profit from partnering institutions by offering foreign qualifications, which in turn may help to build brand reputation or attract more students for economic gain (Healey, 2015). Moreover, academics from the west are concerned by the way TNHE represents the commodification of higher education, and that the pressure of commercial and neoliberal interests is resulting in the subordination of shared cultural, intellectual and societal values (Caruana \& Montgomery, 2015).

Agreements between overseas institutions and Chinese institutions are based on a "nominal emphasis on socialist ideology" (Yang, 2014a, p. 156). Yang argues that the higher education system in China is tightly centralised and so any form of foreign activity is viewed as supplementary during certain stages of learning development. Contradicting this argument, Mok and Han (2016) suggest that the governance of internationalisation activities within regulatory frameworks in China is decentralised based on differing levels of autonomy (enrolment capacity, criteria of admission, and quality assurance). In 2016, there were a total of 577 institutions ( $21 \%$ of Chinese higher education institutions) hosting TNHE. Within this, there is a small group of 79 elite universities with links to research intensive western universities, and these universities have received significant amounts of Chinese government funding. The remainder of the partnerships tended to be set up through overseas validated courses (Montgomery, 2016), which have led to ambiguities around the legal status of foreign higher education activity (Yang, 2014a). Therefore, within TNHE there is a lack of 
consistency within these non-elite partnerships, and the quality of the teaching and learning is left up to the teaching staff and programme coordinators (Yang, 2014a).

My personal involvement and experience corroborates the notion that the affordances of intercultural exchange outweigh the risks of TNHE (Caruana \& Montgomery, 2015). Of particular significance for New Zealand design educators and institutions who are thinking about TNHE is the 2025 'made in China' central government strategy which is impacting design-based student careers (Liu, Liu, \& Zhang, 2018). This policy is changing thinking about education and in particular those involved in design education in China.

\section{The significance of the 2025 'Made in China' political strategy}

The 'Made in China 2025' action plan unveiled by the China State Council in May 2015 signalled the transformative "made in China" to "created in China" ten-year strategy. This ten-year plan positioned strategic design at the centre of business practice in order to move China from a manufacturing business industry with lowprofit margins and "lacking innovation capability, into a world leading manufacturing powerhouse with international competitiveness and the capability of indigenous innovation" (Liu et al., 2018, p. 38). The reference to indigenous innovation implies that China actively seeks to enhance creativity in their people in order to build on aspirations for an innovative, design-led economy to create competitive advantage (Liu et al., 2018). China at the time of this research was five years into the economic direction proposed in the strategy; economic progress was challenging China to rapidly rethink design education for new forms of Chinese talent and originality (Buchanan, 2004).

However, it has been argued there are a number of barriers hindering progress, two of which specifically relate to building design capacity and learning design knowledge (Liu \& de Bont, 2017). Liu and de Bont assert the challenge confronting Chinese educators is that very few design graduates understand strategic design, and there is a lack of learnt multidisciplinary skills which means graduates are unable to tackle a variety of complex concerns with others from different design fields, including 
engineers. These problems are echoed by a group of current design educators who suggest design education is in crisis owing to a lack of critical discourse in which to develop future directions for the discipline (Ho, Li, Liu, \& Wang, 2018). Perhaps other reasons for Ho et al.'s, unease about future directions which seem to be financial and policy driven, may include Caruana and Montgomery's, (2015) arguments about the subordination of shared cultural, intellectual and societal values, and perspectives which examined issues of equality and inequalities related to student accessibility to higher education (Marginson, 2018; Montgomery, 2016).

\section{Pedagogical changes in China}

In 2004 China instigated significant educational reforms which effectively imposed western constructivist approaches on education, including active, experiential and student-centred learning. These reforms, along with their influence, initiated pedagogical change in China (Barratt-Pugh, Zhao, Zhang, \& Wang, 2018). However, unfamiliar educational practices that are introduced into China do not necessarily reflect local cultural values, and so these practices are reluctantly adopted by Asian teachers at the risk of experiencing public disgrace (Hong, 2011). Hong argues that traditional teaching roles establish Asian educators as centres of knowledge, based on the assumption that learning depends on memory, dependent study and "knowledge transmission" (p. 519) through the reproduction of teachers' work. Hong goes on to suggest that changing these established teaching roles can be problematic because western student-centred approaches place the teacher as a facilitator, and intervention happens only during instances of confusion. This reflects western notions of "learnification" in which students take control of their learning, and the role of the teacher is diminishing (Biesta, 2016).

Yang (2014a) raises a number of points about China's internationalisation policies which are correlated with the 2004 educational reforms. The internationalisation of higher education in China has worked strategically along three lines: firstly, it involved sending academic staff and students overseas to study or for research and there was an emphasis on attracting foreign students; secondly, it focussed on integrating international dimensions, for example using foreign textbooks for teaching and 
learning at universities which encouraged development of both bilingual and English programmes; and thirdly, it entailed provision of cooperative TNE programmes with foreign/overseas institutional partners situated within Chinese universities. Therefore, it can be argued that currently, China is positioned within the third wave of this strategy, and the wide reach of TNHE is further discussed in the literature review.

\subsection{A lens for examining VCD creative design process learning}

In order to answer the research questions, which aim to address creative processes in practice, I drew on data from multiple perspectives in a way that acknowledged the cultural and historical context, including tools and artefacts. This required the use of a theory that could capture the complexity of social processes across different systems. A rationale for cultural historic activity theory (CHAT), the theoretical framework chosen for the research, is outlined towards the end of the literature review. But first, through the literature review there are certain problematic artificial categorisations that require acknowledgment. These categorisations relate to east-west and north-south interpretations (Yang, 2014a, b).

\subsection{Artificial categories of east-west and north-south}

The terms 'east-west' or 'north-south' or 'Asian' are problematic, imbalanced and asymmetrical, or, have been seen as simplistic and relying on stereotypes of cultural differences in philosophies and values (Yang, 2014a). Yang's (2014a, b) work in this area powerfully challenges the current theoretical underpinnings of international educational exchange by stating such activities operate in historical contexts of colonisation, in which international education is constructed on the basis of western perspectives. Yang draws on China's negative experiences of western expansion and dominance. He argues contemporary definitions of internationalisation cater to western notions and have been imported to China without consideration of diversity or the potential for difference. Yang points out that there are, "longstanding knotty issues and tensions of westernisation and indigenisation" in determining or understanding internationalisation beyond western ideals, or in other words, without reference to nonwestern countries (p. 153). Doherty and Singh (2005) provide an example of this by 
describing how "the west is done" (p. 53) within international foundation programmes at an Australian university. The authors argue that the university pedagogically positions international students as "outsider or Other, [and therefore], the Otherness of the international student is socially constructed in relation to the category of Western student" (p. 53). Doherty \& Singh's (2005) perspectives about "cultural production" (p. 53) are echoed in the way western cultural orientations tend to lead to conflicts and cultural incompatibilities in the design of TNHE programmes in China (He \& Liu, 2018). He and Liu concluded that power asymetries between the global south hosting countries such as China with its distinct social customs, traditions and histories, and global north "whose education is regarded as at world class level" (p. 270), can lead to a breakdown in transnational partnerships.

Glăveanu (2018) addresses these historical problems of colonisation in relation to philosophical and cultural differences that exist between eastern and western creativity (Lubart, 1999; Niu, 2012; Niu \& Sternberg, 2006). East-west creativity tends to compare Confucian and western beliefs which are suggested to stress "evolution over revolution, community ties over isolated individuals, meaningfulness over novelty, and tradition over change" (Glăveanu, 2018, p. 30). Therefore, when western design and creativity concepts and pedagogies are imported into China, a critical issue becomes apparent: how do western socio-cultural teaching and learning design practices sit alongside Confucian cultural practices? This has led me to consider what literature addresses this. Certainly, the complex relationships between Confucian values and creative design learning in China have emerged as a focus and have shaped teaching and learning at Hunan City University.

For the writing of this thesis, I have used the terms west/western and Asian as these are prevalent in the literature on creativity in China (Glăveanu, 2018; Niu \& Sternberg, 2006). However, it should be noted that in using these terms, I have been mindful that within the critique, the notion of cultural 'hybridity' was also raised (Doherty \& Singh, 2005). This concept positions the classroom at HNCU as a contact zone, whereby a New Zealand teacher such as myself comes into contact with teachers and students from different regions within China; and within this, there resides cultural diversity. Cultures are evolving and dynamic, thus, contact zones such as this, are fluid and 
changing places. Doherty and Singh (2005) suggest that within such places, people with "disparate historical trajectories meet and struggle over issues of representation" (p. 55). Stemming from this, "hybridity and change wrought through contact" (p. 56) with 'others,' is how cultural differences and cultural identity come into being. Therefore, it was critical for me to remain respectful and cognizant about this, through my interactions in the classroom and within written representations of terminology.

\subsection{Research questions}

Although the reach of graphic or VCD design is extensive in everyday life, there is virtually no research on VCD creative design process learning from the west, and research conducted in China or the wider Asian region mirrors this limited field of empirical work. As a result, my research questions have in a sense determined the bodies of literature I have reviewed, including reviews of concepts pertinent to VCD practices. My research questions were focussed on VCD creative design process learning at HNCU, and through the analysis, students' and teachers' perspectives guided my answers to the research questions. Justification for the research questions is provided at the end of the literature review.

\section{Main research question:}

How do VCD students at a university in China engage with creative design process learning?

\section{Two sub-questions:}

1. How do previous and current Chinese teaching and learning practices shape VCD creative design process learning?

2. How do imported and unfamiliar educational practices influence VCD learning activities?

\subsection{Outline of the thesis and chapter introductions}

The current chapter provided an explanation of my personal motivations for this research focusing on how VCD students engage with creative design process learning 
at a single university site in China. The research was situated within a transnational three-plus-one agreement between a New Zealand higher education institution and Hunan City University in China. The significance of New Zealand international education policy, alongside the 2025 'Made in China' political strategy and pedagogical changes in China, underpinned the rationale for the research to be conducted within this context.

\section{Chapter 2: Literature review}

The review which follows begins with an outline of the histories of both western and Chinese graphic or VCD education since the beginning of the nineteenth century. Woven through the histories are explanations of pertinent VCD pedagogies and concepts. The second part of the review focusses on empirical VCD studies conducted in the west and from the wider Asian region, and transnational studies.

\section{Chapter 3: Theoretical and analytical framework}

Key theorists, origins and evolutions of CHAT are presented, along with the way an activity system analytical approach takes into account the way artefact mediated activity and context interrelate. A three-level hierarchy of an artefacts model was developed specifically for the analysis which, in turn, offers an opportunity to extend the affordances of CHAT for future visual creativity research scenarios.

\section{Chapter 4: Research methods}

The research was conducted at HNCU, and the design comprised two phases. The first comprised two case study projects (a branding project, and a cultural project involving illustration design) which were used to explore how creative design process learning occurred in the classroom. The second phase focused on understanding the cultural and historic pedagogical context. Semi-structured interviews were conducted with local teachers at $\mathrm{HNCU}$, and observations were undertaken in Chinese medium classrooms. In total, 24 primary participants were directly involved in both parts of the research and a further 380 secondary participants contributed a range of data for the analysis. 


\section{Chapter 5: Context}

Critical to CHAT is the way in which context interrelates within activity systems. This chapter presents the contextual influences that surround the teaching and learning at HNCU, including: the study site, timetables, pedagogical underpinnings and background contexts for the case studies and teachers' interviews, a situated prior creative learning practice, and the issues involved with working with an interpreter in the classroom and during the interviews.

Three findings chapters follow the context chapter.

\section{Chapter 6: Branding case study}

The branding project involved naming and designing a number of visual identity elements for a hypothetical Chinese tea house including: a logo, a colour palette, and pattern designs for application to paper cups, bags and other potential items.

\section{Chapter 7: Miao case study}

The Miao case study drew from local stories and cultural motifs to inform illustration designs for a deck of playing cards. Teaching a cultural project which drew on culturally significant artefacts from the Miao minority tribe demanded a receptiveness to ways of working that were appropriate and relevant.

\section{Chapter 8: HNCU teachers' perspectives}

The inclusion of the teachers' perspectives was pivotal for the research as it opened up the opportunity to extend understandings about the cultural and historic pedagogical context of the research.

\section{Chapter 9: Discussion}

Key findings from the case studies and the teachers' interviews that where presented in the analysis chapters are discussed in relation to relevant literature.

\section{Chapter 10: Conclusion}

Research contributions, limitations and possibilities for future research are presented, followed by the references and appendices. 


\section{Chapter 2. Literature review}

This chapter begins with an explanation of the way the terms graphic design and visual communication design (VCD) are used interchangeably in the literature, and the rationale for my choice to use VCD for the thesis. Literature about the history of design and design education in the west and China is then critically reviewed. As these histories developed along different lines, the way in which creative design process learning could be fostered at a university in China required a search that explained contemporary pedagogical concepts and practices. This includes design thinking, creativity and, an understanding about the stereotypical creativity deficit belief about students from China (Mullen, 2017).

From here the literature that helps to explain Confucian cultural practices was examined. Although this was not the immediate focus of the research, the influences of Confucianism on creative design process learning emerged during the analysis. The latter part of the review focuses on design and creativity teaching and learning conducted in both the west and in the wider Asian region, and also on transnational studies of design and creativity teaching and learning.

The potential of cultural historic activity theory (CHAT) to illuminate developmental change within creative design process learning, along with the dynamic nature of activity systems and inter-relationships of context pointed to the benefits of employing this framework for this $\mathrm{PhD}$ research. Therefore, a review of the limited studies that used CHAT within creativity frameworks is included.

The chapter concludes by presenting the main research question which guided the bodies of literature I reviewed, including reviews of concepts pertinent to VCD practices. 


\subsection{Graphic design or visual communication design? Three manifestos}

The ways the terms graphic design and visual communication design are used interchangeably in the literature arguably stems from three design focussed manifestos. These manifestos are afforded respect by the design community owing to the way they have helped shape the discipline, both in a theoretical and in a practical sense.

In 1964, twenty-two graphic designers protested about the way graphic design work was used in a consumer society, and they called for their work to be more socially useful (Howard, 1994). The 1964 'first things first' manifesto was written by British designer Ken Garland, and it was published in the form of a folded poster in an industry magazine called Modern Publicity (Howard, 1994). This 1964 manifesto was updated in readiness for the turn of the century when thirty-three visual communicators renewed calls for a change in priorities (Barnbrook et al., 1999). The 2000 manifesto was published through different media channels including the world wide web and industry magazines (Barnbrook et al., 1999; Bierut, 2000). The points of view expressed by the graphic designers suggested that a professional's time and energy was wasted by nonessential pursuits such as using skill and imagination to sell amongst other things "designer coffee . . . sneakers [and] butt toners" (Bierut, 2000, p. 76). These visual communicators argued that their problemsolving skills would be better utilised by drawing attention to "unprecedented environmental, social and cultural crises" through the design of social media marketing campaigns, books, magazines [and] exhibitions” (Bierut, 2000, p. 76). The way the 2000 manifesto changed the original 1964 'graphic designers' terminology to 'visual communicators,' highlights the interchangeability of the terms.

Further debate about the purpose of VCD was conducted by a group of Italian graphic designers in the Spring of 1989 (Illiprandi, 1991). The Charter of Graphic Design Project, presented at the Polytechnic of Milan, was in effect another manifesto which aimed to define the future of the profession. Numerous points were argued in the charter. Since the 1990s, graphic design has taken on the strategic role in the design field (architecture had this role in the 1930s, and industrial design in the 1960s when there was a shift to a consumer society). The profession was a unified whole despite its 
highly segmented and multi-faceted nature (designers, planners, creatives, photographers and illustrators are all involved in this profession). There should be recognition that visual communication is something other than the platitudes about pretty pictures, or it has no quality, or it is relegated to a service function, or the profession has come of age, and that designers have a responsibility to balance business and consumer interests within design communication. This 'manifesto' does not specifically advocate using one term, graphic or VCD over the other; rather it suggested that "communications go hand-in-hand with graphics, and have affected every aspect of life" (Illiprandi, 1991, p. 70).

Therefore, this intermeshing of the terms required me to take a stance. For the purposes of this thesis, I have used VCD for two reasons. Firstly, because the transnational courses at Hunan City University (HNCU) are situated within VCD, and this is the preferred terminology of the qualification. Secondly, in my capacities of both professional and educator, I have always been oriented to the communications side of VCD, and my personal philosophy rejects stylistic graphic treatments being used in isolation for a design work (Kim \& Lee, 2016).

I now turn to a discussion about the emergence of the two separate histories of the design discipline and design education in the west and in China. Various definitions and practices are explained as the discussion progresses.

\subsection{Histories of design and design education}

\section{In the west}

In the early twentieth century, Bauhaus school founder Walter Gropius recognised "new beginnings of a liberal art in design" in which a "technological culture" was required to connect arts and sciences in a way that used experimental thinking and an integration of disciplines to address problems of the present (Buchanan, 1992, p. 6). Experimental thinking alluded to a different way of thinking about things that went beyond objective reasoning (Wang, 2010). The idea of an integration of disciplines is fundamental to design practice, owing to the way in which various fields of design, 
for example VCD or product design, work together or collaborate to solve problems through invention (Buchanan, 1992). Problems of the present in the design field "can be so nebulous and ill-defined that no one knows what is wanted or where to start" (Arnold, 2016, p. 80). Referred to as wicked problems, these problems derive from human global and societal issues (Brenner \& Uebernickel, 2016; Rittel \& Webber, 1973; Wilson \& Zamberlan, 2017). The concept of wicked problems was formulated in the 1960 s by Horst Rittel, a mathematician, who was critical of structured sequential models that moved from problem definition and problem solution (Buchanan, 1992). The issue with a sequential process is that design problems exist in constant flux and part of the problem for designers is that there is no specific starting point or focus on a problem related to a specific discipline. Buchanan (1992) argues design problems can be wicked because they are human-centred, and as a result, there is scope for design thinking to play a part in resolving design problems:

Design problems are "indeterminate" and "wicked" because design has no special subject matter of its own apart from what a designer conceives it to be. The subject matter of design is potentially universal in scope, because design thinking may be applied to any area of human experience. But in the process of application, the designer must discover or invent a particular subject out of the problems and issues of specific circumstances. (p. 16)

The key factors in the quote from Buchanan are design thinking and human experience. Design thinking will be addressed in depth later in the chapter. Within design thinking the human experience is pivotal. Humans are placed at the centre of a problem solving design process (Brown \& Rowe, 2008). Although human-centred concepts had their origins in the western industrial era (human-machine interactions or ergonomics), more recent articulations about human-centredness argue broader interpretations of the term (Krippendorff, 2004). Krippendorff describes an epistemological shift whereby intangible artefacts such as language or play may enable new human-centred design interpretations to be realised. Pillars of this emerging epistemology include "respect for the internal validity of different world constructions, and acknowledgement of the social or cultural role of language in accounting for what people feel, think, see, do or design" (p. 44). 
In the mid to late twentieth century, design education in the west began to respond more broadly to the ideas of wicked problems and human centredness, and the influences of the Bauhaus School became more widespread (Gropius, 1956). The classic western twentieth century modernist design theme 'form' (art) follows 'function' (design) underpinned these new beginnings (Buchanan, 2004). John Arnold introduced the theoretical foundations of design thinking (discussed later) to Stanford University in the 1950s (von Thienen, Clancey, Corazza, \& Meinel, 2018), and the practical or functional foundations of the practice were attributed to Bernard Roth who helped lead the mechanical engineering design division at Stanford (Royalty, 2018). Over time there were demands for a distinct design education epistemology to be established (Archer, 1979; Cross, Naughton, \& Walker, 1981). Simon's (1968) widely cited design definition captured the foundations of such a distinct education by suggesting "the proper study of mankind [sic] is the science of design, not only as the professional component of a technical education but as a core discipline for every liberally educated man [sic]" (p. 83). Ten years after this definition was published, Archer (1979) asserted a third area of education existed in modern education alongside numeracy and literacy, owing to the way practical subjects such as art or craft embraced an awareness of the inherent issues of the material culture. What this meant is that within this area of learning, making and doing activities are used to attend to human problems, including ecological, environmental and societal issues. These problems have an effect on the material culture, which is anything produced by humans, including physical spaces, objects and communications (Archer, 1979).

However, there still exists a pervasive unease about design education (Wang, 2010). Wang's article argues that design education is marginalised in relation to a university education, owing to its focus on social relevance and sacrifices of intellectual rigour:

In order for design education to become more rigorous - and more academically respectable - it must either become more rational or it must embrace a new paradigm that values creative experience. (p. 173)

An understanding about complexity within established studio settings and contemporary pedagogical practice is argued by Wang to offer design education the 
rigour that is required. This paved the way for the relevance of a complexity theory for design education. According to Wang (2010), within complexity theory there is no distinction between the "knower and knowledge" (p. 178) which challenges Cartesian dualism (mind and matter as distinct entities) owing to the way in which a student uses educational experience to potentially create subjective values and meanings. In other words, creating new knowledge is constructed from a wide variety of phenomena. Curiosity, discovery and experience is an open-ended process which operates within human systems that are even more complex and anti-rational than is seen in contemporary design education (Wang, 2010). However, ironically, the reality of teaching students within a complexity framework is that it is too complex.

\section{Origins of contemporary pedagogical practices}

The academic discipline of VCD teaches a global language which by its very nature must communicate concepts and ideas to people through visual or communication means, for example, purchasing products through the internet or by designing multilingual signs to direct people at a busy international airport (Buchanan, 1992). Schön's $(1984,1987)$ reflective practice theories and the studio-classroom have become a core component of design education. Ledewitz's (1985) analysis-synthesis model has also shaped practices that support student learning in the studio. Drawing on Ledewitz (1985), the VCD studio classroom has three pedagogical objectives: to teach new skills, to teach visual languages, and to teach students "designerly ways of knowing" (Cross, 2007, p. 17). New skills include technical learning in the use of design software, for example InDesign or Photoshop. Teaching students about visual languages involves “examples, or precedents" (Cross, 2011, p. 147), and studies of signs and symbols, for example, colour and typography to understand how meaning can be made in design communications (Crow, 2003). Designerly ways of knowing involves teaching students how to move from an 'analysis' (problem-defining) stage, which is a rational, planning stage, to the 'synthesis' (problem-solving) stage, which is creative, intuitive, and takes great courage because it asks the student to make a "leap into the dark" (Ledewitz, 1985, p. 4). Ledewitz's analysis-synthesis model may be used in the classroom in different ways, for example, some educators may tend to gravitate towards an emphasis on well-defined planning and introduce quick fire 
exercises in the hope that students can move into an unstructured synthesis stage to complete a project. Ledewitz (1985) descibes how educators become "more responsive to student-initiated moves" (p. 3) during the synthesis stage, are less inclined to impose predefined procedures on the student, and use individualised or group critique.

Implicit in Ledewitz's (1985) analysis-synthesis model is an epistemology of practice for educating reflective practitioners (Schön, 1984, 1987). Schön (1987) descibes "reflection-in-action" (p. 44) as a process in which teachers impart information through a "language of designing that combines drawing and speaking" (p. 58). Through this process, a reflective conversation addresses the problem that a student is grappling with, and an order is imposed on uncertain or complex design situations. It is common for studio-based practices such as this, which are used in professional practice, to be replicated in design education and these practices are assumed to help the progression from novice to expert (Cross, 2011; Schön, 1984, 1987). Cross suggests that a minimum ten-year period of practice and dedicated application of a performance are required for design expertise to fully develop. But there is still limited understanding of how and when students move from novice to expert, and what triggers the shift (Cross, 2011). Prising apart what designers know about their design processes and decisions remains largely tacit knowledge; it is difficult to explain and design educators must find ways to explain why things may work better - like this, or this - within a studio-based practice learning system (Cross, 2007).

Polanyi' s (1962) theories of tacit knowledge are evident in both Cross's and Schön's perspectives. Polanyi contributed theoretical stances whereby the "things that we know but cannot tell" (p. 601) are derived from the idea of tacit knowledge. Tacit knowledge is a kind of knowledge that can be viewed as ephemeral or even invisible and is hard to articulate. This idea can be applied to skills in general; however, what designers know about their own problem-solving processes remains largely tacit knowledge and difficult to verbalise (Cross, 2007):

In the design studio . . . the coach's showing and telling are interwoven, as are the student's listening and imitating. Through their combination, students can learn what they cannot learn by imitation or following instructions alone. 
Each process can help to fill the communication gap inherent in each other. (Schön, 1987, p. 111)

Schön captures the way a teacher models and a student imitates practice through active or think aloud conversations, and design work is gradually resolved through experiences of trial and error. Thus, the notion of learning through imitation and working with a knowledgeable other resonates with Vygotsky's (1978) zone of proximal development (ZPD). ZPD is when the distance between actual and potential development is determined by independent problem solving conducted in "collaboration with more capable peers" (p. 86).

Similarly, this way of working is congruent with Dewey's (1938) conceptualisation of experiential learning as an exploratory process. Experiential learning has a long history. Systems for experiential learning include traditional trade and craft apprenticeships. In nineteenth century France, this included the "atelier system of the education of architects at the École des Beaux-Arts" (Wang, 2010, p. 175). Dewey's (1938) argument in support of a theory of experience was picked up by Kolb (1984) in his development of Experiential Learning Theory. The theory depicts an iterative learning cycle in which learning based on experience provides a way to grasp and transfrom learning. Kolb suggests four stages that can underpin pedagogy: concrete experience, reflective observation, abstract conceptualisation, and active experimentation. It is suggested that these pedagogies may be developed through two learning process dimensions which complement each other. The first places concrete experience events at one extreme, and abstract conceptualisation at the other, and the second dimension places active experimentation at one end of learning and reflective observation at the other.

A design process may follow either a linear or iterative pathway (Cross, 2011). Linear may be understood as following a series of steps based on a set of specifications. Within an iterative process, whilst there may exist a series of defined steps towards a final outcome such as research, idea generation, experimentation and testing, this process is in fact messy, tangled and in constant flux. For example, when in the final design stage, it may be necessary to return to further research or 
experimentation to better resolve the problem. Within this state of flux, a range of design possibilities is produced, providing the scope for multiple solutions with no one solution necessarily better than another (Buchanan, 2004). Design thinking is a prescriptive design process and is a practical tool to use with students in the classroom because it helps in articulating and directing the iterative stages of a design process (Cross, 2007, 2011).

\section{Design thinking}

Design thinking (DT) is a signature design pedagogy which has been advocated as a powerful process model that enables design creativity and innovation to emerge (Cross, 2011; Davis, 2016). The Hasso Plattner Institute of Design, commonly known as the Stanford d.school (design school), introduced the most recognisable DT model for design education. The Design Thinking Bootleg cards outline the current generally accepted design thinking process procedure. The five steps or modes set out in the Bootleg cards are empathise, define, ideate, prototype, and test.

Brief descriptions of each of these steps are as follows: 1) empathise, which involves empathy, observation, engagement and immersion with particular users, and problems that require solving, 2) define, involves unpacking empathy in order that the problem be defined into needs and insights, 3) ideate, promotes multiple idea generations, or radical design alternatives which involves "flaring" instead of "focus," 4) prototype, takes on physical forms through inexpensive and low resolution ways, to further explore possibilities, and 5) test, involves an iterative process of gathering feedback, refining solutions and continuing to learn about user needs (Stanford d.school, 2018).

There has been widespread adoption of DT from disciplines other than design, for example, business and general education in order to promote innovation (Orthel, 2015). In response to the spread in the adoption of DT, the DT courses at Stanford d.school were developed to provide DT education specifically for non-designers with the intention of creating innovative thinkers rather than creating particular innovations (Rauth, Köppen, Jobst, \& Meinel, 2010). 
The problem with this widespread adoption and generalised practice that has emerged is that DT is falling short at equipping design students for new professional directions (Davis, 2016; Kimbell, 2011). Dorst (2011) concludes that a 50-year theoretical and practical understanding of designerly abilities is misrepresented in the way design thinking is used in a generic way to label "vague creative activities" (p. 531). Royalty (2018) argues that when DT is placed in the hands of designers, there is potential for significant innovation to emerge and that design students tend to emerge as leaders. This is owing to a pedagogy which aims to master the analysis to synthesis gap (Ledewitz, 1985). Although there is little evidence to support their assertions, scholars have also suggested that some designers develop abilities which combine extraordinary aesthetic sensibilities and deep sensitivities towards ethnography, pattern recognition, and visualisation, that are well beyond the norm (Ogilvie \& Liedtka, 2011 , p. 5). This is where the creativity of design students comes into play. Creativity is recognised as a critical component within design practice owing to the way it draws on experimentation processes and abilities of fluency and flexibility for problem solving (Orthel, 2015).

\section{Creativity}

The tenets of creativity theory were captured by Runco (2004) in an overview which defined the phenomenon in four ways: personal creativity (personal characteristics, traits, motivation), press (pressures on creativity, influence of environment and sociocultural factors), product (creative outcomes), and process (systems theories, historical and associative processes). The field of research in this area is vast and beyond the scope of this thesis; however, there is a requirement to understand the role of individual creativity and the influence of the wider social context for design practice and education. Kaufman and Beghetto (2009) characterised individual creativity under their "Four C Model of Creativity" which comprised four categories: Big-C (genius or eminent level), little-c (everyday expressions), pro-c (professional level) and mini-c (creativity in learning) (pp. 1-2). This model was advanced by Csikszentmihalyi (2014) who introduced a systems model of creativity whereby three interrelated systems comprising person, field (critics or academics), and domain (art or science) work together to produce and evaluate innovations which reflect current socio-cultural 
issues. This in turn provides a new starting point for the next generation of people (Csikszentmihalyi, 2014).

\section{Social cultural conceptions about creativity}

Early cultural-historic activity theorists believed that humans adapted their actions to external conditions (environment) in the production of human activities. The genesis of human activity derived from social and cultural needs (Leontiev, 2005; Leontyev, 2009)*. Shared "ideas, beliefs, knowledge and emotions" are both internalised and externalised through a creative process (Moran \& John-Steiner, 2003, p. 63). For Vygotsky (2004), embodied cultural artefacts are learnt in childhood. Vygotsky suggested that many insignificant drops of "anonymous collective creative work [lead to the] overwhelming majority of inventions" (Vygotsky, 2004, p. 11). For example, this could represent something as simple as preparing a meal. The way a meal is first prepared depends on social and cultural customs which may eventually elevate a particular cuisine to renowned status, for instance, French cuisine. When something does become renowned, questions about originality begin to enter conversations.

\section{Originality}

The question of searching for original ideas or in trying to ascertain how original ideas emerged was addressed by Wallas (1926) who identified four stages of the creative process: preparation, incubation, illumination, and verification. He poetically described illumination as akin to "conceptions rising into a field of consciousness like a flash of lightning or the flight of a bird" (p. 80), and within this mysterious phase, moments of inspiration are characterised by a strong emotion. The idea that creativity is illuminated like a metaphoric lightbulb flash, a eureka feeling, or as an a-ha (design epiphany) moment is still a contemporary view (Amabile, 1996; Lubart, 1999; Tregloan, 2010). Articulations of illumination include the idea of serendipity, and combinations of magic and logic being the key ingredients for design strategy and new

\footnotetext{
* Through this thesis, different spellings/translations for the same theorist listed here; A.N. Leontiev, A. N. Leontyev and A. N. Leont'ev have been encountered. Therefore the way in which various authors intended is reflected in the in-text citations.
} 
innovation (Neumeier, 2005). However, critics challenge outdated notions of an illumination phase by believing that there is little conscious awareness of the way creativity may appear in unexpected ways (Amabile, 1996; Howard, Culley, \& Dekoninck, 2008; Lubart, 1999). In a recent professional journal for architecture and design, illumination was linked to the "originality syndrome" described as an “imposed ambition” for students (Barr, 2018, p. 1). Barr asserts that students are taught to believe that original designs appear unexpectedly, yet most educators rely on precedents or artist models for learning success.

Connections between originality and creativity were further addressed by psychologists in the 1950s. Guildford (1956) argued the study of creativity was neglected, and his theories on imagination, originality, thinking and the testing of creativity provided a starting point for further research in this area. Guildford outlined attributes that he considered were found in a creative personality, including; sensitivity to problems, ideational fluency, novel ideas, flexibility of mind, ability to synthesise and analyse, and an ability to organise complexity. Guildford's attributes continue to be reflected in current conceptualisations of creativity, for instance, with three emotion-centred variables relating to creative minds, recently described as daringness or boldness in the face of risk, enthusiasm and drive for problem-solving, and engendering self-confidence or belief in oneself (von Thienen et al., 2018). Two further creativity theories are discussed next; the first situates originality and creativity connections within an historic background.

\section{Two creativity theories}

Glăveanu (2018) delineated the history of creativity in three ways that provide a holistic link between design and creativity. The first type was grounded in Romantisim, an intellectual and artistic European movement beginning at the end of the $18^{\text {th }}$ century. The movement is characterised by spontaneous and self-expressive artistic creativity resulting in highly original and novel outcomes. The second emerged from the period of Enlightenment and the first Industrial Revolution in Europe (c.1760-1850), a time when Eurocentric and American world views were aligned with exploration, rationalism, problem-solving, and invention. This enduring type of "functional creativity" (p. 28), which uses insight rather than inspiration, provides 
practical solutions for complex human challenges. The third type of creativity is based on craft, and here, guided participation, development of skill and practice lead to mastery, for example, a mother teaching a child how to sew (Glăveanu, 2018; Rogoff, 1995). Key to this idea is the way in which sociocultural traditions and the wider context is central to the creative process. The relevance of imitation and repetition, and use of precedents and mentor support is critical to the enablement of learning, and these attributes are assembled in such a way that is unique to a particular social and cultural context.

Tikhomirov's (1999) theory of creativity presents a Russian perspective on originality. In his theory, comparision, reproduction, assimilation, and copying are the functions of non-creative activity, whereas construction, generation and creation of the new are the most important operations for creative activity. Tikhomirov draws on Guildford's (1956) story about an American dean who in trying to encourage originality, graded an essay highly in which a student had compiled transcribed lecture notes reiterating the deans' pet ideas. Reproduction of facts was also critiqued by Guildford in the 1950s, who went on to propose creativity tests be designed and conducted because the commonly used intelligence tests were unsuitable for comprehending creativity.

The Torrance tests, which developed from Guildford's ideas, used systematic and disciplined testing methods in "figural test batteries" which measured creative personality attributes including originality, flexibility, fluency and elaboration in school children at kindergarten through to graduate school (Torrance, 1972, p. 4). Torrance's (1972) guide provides examples of tests and how to score the "creative strength [of each of the categories, and it is argued that] more intellectual energy is required to give responses that go beyond what is learned, practised, habitual, and away from the obvious and commonplace" (p. 13). For example, children would be given the title "a fat mouse in cheese heaven" (p. 16) and the more elaborated the detail (ears, mouse, tail, holes in cheese, cheese crust, thickness of cheese), the higher the score. 


\section{Summary}

In summation, through the nineteenth century and until now, design education in the west has been challenged, primarily because it does not sit comfortably within a paradigm in the way science and art do (Archer, 1979). Yet pedagogies such as design thinking and studio-based practices used by design educators in the promotion of creativity and innovation are adopted by other disciplines to address the myriad of contemporary human problems (Archer, 1979; Rauth et al., 2010). The Chinese government recognised the importance of creative design education within this context when the 2004 educational reforms were introduced (Barratt-Pugh et al., 2018). The 2015 "created in China" strategy further solidified this position (Liu et al., p. 38). I now turn to a discussion about the history of design education in China in order to understand the context in which creative design process learning exists. It should be noted in the next section that there were constraints on sources owing to limits on reading material that were published in English.

\section{In China}

At the beginning of the early nineteenth century in China, design education favoured graphic design, which is called Zhuang Huang, or decoration (Wang, 1989). Wang's three decade-old retrospective on China's design history is a fascinating walk through Chinese political history and its effects, along with those of post-modernism on graphic design. Wang began the retrospective by arguing that it is necessary for design in China to "catch up with everything new" (p. 49). He attributed the reason China was then in catch-up mode was owing to more than a century of difficulties, including:

...a traditional hostile attitude to all non-Chinese cultures, an attitude formed in the late Ming and Qing dynasties, civil wars including the Taiping Rebellion in 1860, and the Boxer rebellion in 1900, foreign agression and expansion in China since the 1840s, two Opium wars with the British at the turn of the nineteenth century, the 1931 Japanese occupation through to the end of 1945 , the civil war between the communists and nationalists in1946, communist government activities from 1949 to the late 1970s including the cultural 
revolution from 1966 - 1976, and the isolation of China after it broke relations with Russia in the 1960s. (p. 52)

Wang points out that after the 1860 Taiping Rebellion, in the interests of military defence, several hundred students were sent to the west to learn about western technology, and from this time examples of Chinese indigenous graphic design included examples of cigarette, matches, and soap packaging. Later, in the 1930s, design offices were set up in Shanghai for a short time. During that period, graphic designers used a traditional linear brushwork illustration style to depict the Shanghai beauties for a range of products, for example, soap and candy. Furthermore, the influences of Art Nouveau, Art Deco, and De Stijl movements were seen in packaging Bao Zhuang. Within typography, the Hei Ti (black style) designs were influenced by western sans serif typeface developments, and German expressionism influences were seen in the woodcut designs used for book covers and illustrations.

In the 1950s, the Ministry of Culture directed publication design Shu Ji Zhuan Huang houses to produce propaganda, and graphic designers produced cover designs, illustrations, layouts, and typography for these books. Commercial graphic design at this time did not exist because it was considered symbolic of western lifestyles that encouraged unnecessary waste. However, in cities such as Shanghai, Beijing and Guangzhou, in the late 1950s through to the end of the cultural revolution in 1976, advertising agencies Guang Gao (which means announcing to the public) were formed but they were not commercial per se. Graphic designers in these agencies were directed to copy pre-prepared portraits of Mao and other political leaders onto posters and large billboards. The only place for design freedom during this time was on the walls of buildings alongside railroad tracks. Hand-painted advertisements for products such as Chinese traditional medicine, tooth brushes and tooth paste were simple, "a big logotype, the name of the manufacturer, and an illustration" (Wang, 1989, p. 68).

Apart from "fragmented attempts" at graphic design, Wang (1989) argued that China's modern design movement did not really get underway until 1979, and once it did get underway, ten years later there was a propensity for designers to "copy and imitate foreign design" (p. 76). According to Buchanan (2004), the respected Chinese scholar 
Zhou Zhi Wang** introduced new ideas to modernise design education in the 1980s. These ideas moved the traditional arts and crafts education system towards the classic twentieth century western modernist design theme - form (which aligned to creative art practices) follows function (which aligned to design practices) - and this thinking coincided with the market economy that emerged at this time within China (Buchanan, 2004). From the 1980s onwards, three brief economic phases followed in which creativity was a relatively new focus (Liu et al., 2018). First, during the 1980s, the industry economy focused on establishing and creating manufacturing capabilities with a push for technology driven strategies. Next, the experience economy of the 1990s favoured developing technologically innovative capabilities with a market-pull strategy. From 2010 onwards, political policy strategically repositioned China's economy, and as a result there has been a rapid shift from production/manufacturing to that of design/innovation (Liu et al., 2018).

Prior to the 2004 educational reforms, as China was about to be accepted into the World Trade Organization, design scholars were arguing for change. Shung-Yu and Yuet-Ngor (2001) argued that design education needed to adapt in order to compete with the potential for foreign competition within the Chinese marketplace. These authors were involved in teaching at design schools in Beijing, Shanghai and Guangzhou. They gathered data through their visits to those design schools, attending conferences, and by sending out 100 surveys to businesses in each of the three cities. Key findings from their research included: Chinese design educators placing noticeably more emphasis on the "product rather than the process of designing the product" (p. 173), design courses conducted in fine arts departments modelled on the atelier system which limited cultural, human and commercial design applications, and teacher-centred systems emphasising "copying and mastering the styles and techniques of master teachers" resulting in students accepting this as normal practice (p. 176). Among recommendations Shung-Yu and Yuet-Ngor (2001) made to improve

\footnotetext{
** This is the same Wang who wrote the retrospective. Buchanan refers to him as Zhou Zhi Wang but he is also known as Shou Zhi Wang.
} 
outcomes for design education was the suggestion to introduce creativity courses and to use creativity tests, for example the Torrance tests, rather than relying on drawing skills for entry into design schools.

\section{Design thinking}

Buchanan's (2004) keynote address to the first design education conference in China questioned how philosophical roots of art and design could lead to an original version of design thinking that is not an imitation of western approaches. He suggested that the "implications of this relocation of design are still unfolding" (p. 35), that design thinking approaches may change, and although the west and China face similar problems, for example climate change, solutions are not required to be the same (Buchanan, 2004). The problem with importing a western creative design process model such as DT into China is that the model is not a Chinese model. Buchanan argues that DT does not account for Chinese art and design education that is intertwined; art and design are so closely connected in China, that eastern educators find the western division of arts and design "strangely artificial" (p. 33).

Keane (2006) argues that part of the problem, not only for western educators but also for introduced pedagogies such as DT, is the assumption of the universality of western ontological perspectives about creativity and creative design processes. These include the view that creative design learning from liberal democracies is unlikely to work in China. Creativity is seen as a tool for operating soft power in which to propagate Chinese Communist Party ideology (Lin, 2019). Lin suggests creative workers in Beijing have to work within state expectations of conforming to a politically aligned creativity, whilst managing personal creative notions and career aspirations. Keane (2006) argues "political correctness and a fear of overstepping the line" (p. 286) work to constrain creativity in socialist China. Perspectives such as these may accompany students when they leave China. In a related vein, Zhang (2017) suggests arts and humanities students from China are disadvantaged when it comes to developing critical thinking skills, knowledge and disposition within British universities because they have not learnt to question or reason the absolute truth of the doctrine. Positioning this as a development since 2004 when a new China-wide curriculum was introduced, 
Zhang argues students "are treated as passive targets of ideological and political control" during their education (p. 862). The foundations of Zhang's argument lie in assessment of the compulsory four treasures modules (Fundamentals of Marxism; Mao Zedong's thought and Chinese characteristic socialism; Outline of modern Chinese history; and Moral, legal and civic education) (Zhang, 2017). To assess competencies in the modules, imported aptitude testing methods use multiple choice and predetermined answers, which is asserted to deplete abilities to formulate an argument, which in turn has sacrificed creative thinking (Niu, 2007). Arguments raised about this apparent stifling of creativity have come about because Chinese students go through the education system being tested - and mass testing requires structured tests. The cumulative effect of this regime, not just in arts and design but throughout all subject areas, draws attention to the creativity deficit belief and stereotypes about students from China, which is discussed next (Mullen, 2017).

\section{The creativity deficit belief and stereotypes about students from China}

Stereotypes - assumptions that are made about a whole category of people - can be harmful when exercised, as they create prejudice, discrimination and outdated views (Ryan, 2016). Yet, perspectives about the creativity of design students from China tend towards the negative, and comparative studies are still reasonably common (Ceppi, 2017; Niu, 2012; Yi, Hu, Scheithauer, \& Niu, 2013). For example, Chinese students studying abroad were compared to German students in a study which questioned how bicultural and bilingual experience could accelerate artistic creativity (Yi et al., 2013). These authors asserted that "German participants ... produced more creative and aesthetically pleasing artwork than did their Chinese counterparts" (p. 99), and there was general agreement between Chinese and German judges on what constituted creativity. Another example by a western teacher working in a Sino-Foreign joint venture school in Shanghai, described design students as lacking in critical and creative thinking skills (Ceppi, 2017). Mullen (2019) outlines three stereotypical reasons for the creativity deficit. The first relates to history. The historical imperial examination (the keju), which tested memorisation, is still honoured today through a transmission learning process in which schools and the government prize achievement. The second reason is attributed to failing individual achievement within China's test- 
centric culture, resulting in shame, suicide, and large numbers of students seeking an education outside China's borders. For high school students, the infamous "gaokao" or the government college entrance examination is described as brutal, as it determines eligibility for higher studies (Mullen, 2017). Mullen attributes the third reason for the creative deficit belief to popularisation. The notion that the Chinese are not creative is perpetuated by global news. This kind of reporting is not helped by perspectives that are held by Chinese educators themselves. Ho, Li, Liu, and Wang (2018) suggest creativity is limited by a "lack of curiosity and self-confidence [which] is common among students" in design education (p. 85).

A growing body of literature is now challenging the creativity deficit belief (Heng, 2018; Hilton, 2016; Li \& Wegerif, 2014). Language limitations are argued to accentuate the creativity deficit belief. In one study the influence of language proficiency compromised conceptual thresholds for Chinese design students in the United Kingdom (Hilton, 2016). Heng's (2018) study adds weight to Hilton's argument by pointing out that Chinese students have to adjust to very different sociocultural contexts when they leave China, they are challenged by student-centred teaching, and have to think like a "westerner" (p. 22). Heng argues different is not deficient, and that Chinese students possess agency which may become enabled over time. Agency, arguably, may be attributed to the way in which a Confucian influenced creative education is misunderstood, which is argued to be paradoxical as students from China may become highly conceptual despite their background (Li \& Wegerif, 2014; Mullen, 2017). What is lacking from stereotypical perspectives is a recognition that the Confucian tradition for teaching through a dialectical approach is one which critically considers multiple view-points (Li \& Wegerif, 2014). This is just one aspect of Confucian teaching and learning practices. In the next section I discuss the way both contemporary Chinese life and education are influenced by Confucianism.

\subsection{Culture and Confucianism}

Niu and Sternberg (2006) propose that the philosophical genesis for Chinese culture derives from circular and successive reconfigurations of nature, which "may or may not bring new forms of everything” (p. 35). This is different, they argue, to western 
ontologies which privilege god/gods and divine origins in the creation of new and novel things from nothing. Niu (2012) argues that in Chinese culture the inception of new things is not viewed as a breakthrough; rather, they are extensions or continuations from the past or present which may be considered appropriate for changing contexts.

Contemporary Chinese culture possesses features from both its own traditions and western influences alongside moral goodness (Niu, 2012). Niu argues individual worth (moral goodness) is determined by how individuals work on self-development or selfcultivation. This is important in the development of one's self, and in developing one's contribution to the collective or group. Therefore, it is suggested that people from China are more concerned with confirming and maintaining harmony with others, and thus appear to be "introverted, submissive, and close minded, whereas western societies are more concerned with individualisation and so appear critical" (Niu, 2012, p. 274). As a result, it is suggested there is natural opposition to individual creativity as the values associated with moral goodness in Chinese society may appear to prohibit creativity development (Niu, 2012; Niu \& Sternberg, 2006).

Yet, creativity was central to ancient Chinese philosophy for the "perfection of one's humanity," and individual creativity was required to follow a process of "selfcultivation toward enlightenment" (Niu \& Sternberg, 2006, pp. 30-33). To achieve enlightenment, two schools of thought developed in Chinese philosophy, Confucianism and Taoism, both of which are important cultural legacies for its people (Chun, 2018). Confucianism began in the Han dynasty, second century BC, and developed for centuries through to 1911 when the Qing dynasty collapsed. In more recent years Confucianism has been accorded an important place in China, including in the education system. Given this long history, it is notoriously difficult to define owing to multiple interpretations about its multifaceted nature, and also, its predominant use of metaphor (Sigurðsson, 2017). Lao Tzu, attributed as the founder of Taoism, encouraged the practice of losing oneself in meditation and doing nothing, which contrasts with Confucius, the founder of Confucianism, who advocated a gradual creative learning process (Niu, 2012; Niu \& Sternberg, 2006). Therefore, philosophically, Confucianism considers creativity as a slow process that relates to 
change and the experience of "interacting with the context" (Niu, 2012, p. 279). The practice of creative learning as a slow and gradual process requiring much effort, repetition, and attention from a young age has been critiqued as resulting in predictable creative responses (Niu \& Sternberg, 2006). It also stands in contrast to the fast moving way of working associated with multiple iterative design thinking processes (Brown \& Rowe, 2008).

Confucian educational thought is based on systems of "Rite (Li)" (Chun, 2018, p. 108). In brief, the abstract idea of Rite encompasses social interactions of humans, nature and material objects (the context) within codes of hierarchy and morality (Chun, 2018). This, in turn, means Rite influences the context of creative process. However, the cultural priority of Confucian education is to progress through a hierarchical structure of learning to attain "junzi" (Hung, 2016, p. 85). Junzi is a noble or superior person who achieves the highest level of knowledge "pertaining to ethics, morality and virtue," as distinct from tangible knowledge related to literary studies or labour (Hung, 2016, p. 87). These last points suggest that a Confucian education focusses on teaching and learning within the context of relationships and moral responsibility.

A dialectical approach is promoted in Confucianism where a silent inner dialogue and reflection take place (Li \& Wegerif, 2014). Li and Wegerif explain this by discussing the way "insights emerge out of the inter-illumination that occurs when multiple different perspectives are held together in the same space” (p. 25). Sigurðsson (2017) called this a "transformative self-critical attitude" (p. 132) which operates in continously critical cycles. This dialectical approach was based on a belief about opposite phenomena or "universal opposition among things" (Chun, 2018, p. 71) in which things are treated as whole, rather than a part. This idea is recognisable in the concept of yin and yang, which attempted to reconcile the diversity of phenomena, for example, more and less, or fast and slow into a type of harmony, which eventually "leads to growth and development" (Chun, 2018, p. 29). It is suggested that this distinct characteristic of Chinese education is not always readily visible in the classroom (Li \& Wegerif, 2014). Added to this, Li and Wegerif assert students who come from a Confucian educational background are taught to accumulate and memorise rather than construct or speculate on knowledge and to not express their 
understanding about their learning unless and until it is really necessary. In contrast, Nui (2012) highlights the positive dimensions of the Confucian ideal of self-cultivation as achieved by building character, being open-minded, and by controlling personal emotions and behaviours when working with others.

In summation of this section the next excerpt, attributed to Confucius, provided a glimpse into a creative process that is distinctly Chinese:

The principles of higher education consist in preserving man's [sic] clear character, in giving new life to the people, and in dwelling (or resting) in perfection, or the ultimate good. Only after knowing the goal of perfection where one should dwell, can one have a definite purpose in life. Only after having a definite purpose in life can one achieve calmness of mind ... Only after one has learned to think, can one achieve knowledge. There are a foundation and a superstructure in the consitution of things, and a beginning and an end in the course of events. Therefore to know the proper sequence or relative order of things is the beginning of wisdom. (Confucius, 1954, p. 354)

This excerpt tells us that the ideal or aspiration for perfection specifically relates to purpose which is to attain the highest level of morality or goodness and what the role of education is. This is different to another interpretation of perfection which relates to something being faultless through the actions or process of improvement. Furthermore, in the excerpt, an educational process is hierarchical, as suggested by the relative order of things on perfection, morality, and hierarchy.

\section{Conclusion}

The literature highlights that design educators in China have had little opportunity to develop an indigenous discipline owing to the way western methods have been both imposed and adopted (Wang, 1989). Western perspectives on Chinese students and creativity have led to problematic creativity deficit stereotypes (Ceppi, 2017; Niu, 2012; Yi, Hu, Scheithauer, \& Niu, 2013), and there is limited understanding of China's diverse cultural traditions and the role that Confucianism plays in contemporary life and education in China (Li \& Wegerif, 2014; Mullen, 2017). 
The next part of this chapter reviews empirical research on design and creativity studies from the west and from the wider Asian region including Confucian heritage countries outside China, along with an examination of the transnational education literature.

\subsection{Empirical design and creativity research}

\section{Western studies}

Given the extensive and ubiquitous nature of VCD in everyday lives, it is surprising how few studies have been conducted in this area. Saris (2020) identified six empirical studies within the field of western graphic and/or VCD education that related to creative design process learning; specifically, research conducted within engagement, activity, and process areas. I begin with a New Zealand study in which the "physicality of craft-led thought" is argued to be important for graphic design education (Ings, 2015, p. 183). Postgraduate expert designers with industry experience conducted practice-led research which evidenced handmade (tactile) process combined with digital processes. Ings suggested that this interpretation of craft is a forward-thinking way of engaging and or re-engaging with both happy accidents of discovery (for example, embracing mistakes or marks produced through screen printing and combining with emerging technologies), and social learning. Encouraging social learning and critique within education communities in this way is critical to the way future graphic designers may embrace the potential of creative ambiguity, disruption, risk taking, and curiosity (Ings, 2015).

Continuing with findings from the Saris (2020) review, Fontaine's (2014) study alone used the DT process within the pedagogical design of an environmental graphic design project at a university in America. A range of two and three-dimensional museum exhibits was designed to enhance visitors' learning experiences. Fontaine argued that by incorporating DT into the project, students developed skills focussed on problemsolving first and image-making second, thus, moving the focus of learning to the design of experiences rather than design of objects, which in turn would be expected of twenty-first century design graduates (Fontaine, 2014). Ideation was mentioned in a 
New Zealand study which evaluated a 30-minute client-based design exercise (Laing \& Masoodian, 2016). Eighteen graphic design students in Laing and Masoodian's mixed method study were exposed to the influence of visual imagery on creative design process. Findings revealed that student exposure to imagery during the ideation phase had little effect on measurable creativity of outputs (Laing \& Masoodian, 2016). Locating any further empirical VCD studies that used DT as a pedagogic model proved difficult and no further studies were found.

The preceding discussion reveals a significant lack of western research that connects DT with VCD education. Despite design research gaining momentum in the last 20 or so years, graphic or visual communication design research has lagged, possibly owing to naming or nomenclature issues associated with the discipline (Walker, 2017). In contrast to the lack of empirical research, an extensive range of theoretical and pedagogical essays has been published (Heller, 2015). Likewise, the sub-disciplines within graphic design research have received much attention. Walker (2017) documents the extent of this, identifying social, historical and cultural trends in illustration, posters and maps, national styles in typography and individuals who have made significant contributions to typography and branding. Despite this, the apparent lack of interest in graphic design research more broadly is attributed to graphic or communication design as a discipline being referred to as "supplementary or additional" (p. 549) to other design disciplines, for example, emphasis may be placed on the design of a 3-dimensional product, and any packaging or communications about that product is added later. Yet, the role of a graphic or visual communication designer is as wide as it is varied:

Graphic designers try to illuminate or explain things using graphic language, which may be verbal, pictorial, or schematic, and presented on paper or screen. Graphic designers work with illustrators, photographers, coders, editors, writers, and others involved in the graphic [or visual] presentation of language. And, as with all designing, it is a planning and problem-solving discipline, concerned with purpose, process, visual judgement, engagement with users, and circumstances of use. (Walker, 2017, p. 549) 
Although a student may begin their education specifically within the VCD area, it is common practice for other design fields (for example architecture or product design) to become an integral part of a design scenario or situation as the novice moves towards expert (Cross, 2011; Schön, 1984, 1987). This raises the notion of collaboration, and terms associated with this. Multidisciplinary, means two or more disciplines work together but there is no "adoption or integration of concepts, methods or theories," and interdisciplinary, begins with an adoption of multiple disciplines resulting in the "creation of a reciprocal hybrid practice" (Jones, 2010, pp. 155-157). By way of an example of research in this area, an American study examined 93 first year college students from a broad range of majors who were enrolled in a general interdisciplinary creativity course focused on innovation (Lee, Portillo, \& Meneely, 2019). This 16-week interdisciplinary creativity course wove tenets of creative theory through the learning, including; Amabile's (1983) intrinsic/extrinsic motivation hypothesis, Kaufman and Beghetto's (2009) Four C Model of Creativity, and Csikszentmihalyi's (1990) concept of flow. Flow is likened to a meditative state as individuals move with and through an activity (Niu \& Sternberg, 2006). A transformative learning lens was applied in the analysis which found that students' attitudes towards creativity learning had changed, and they became motivated and confident in their actions. Within the findings, the notion that anyone can be creative, and can contribute original ideas is a direct reflection of Vygotsky's (2004) ideas about anonymous collective creative work (drops of creativity) resulting in innovation, and little-c creativity in which creative potential is widely distributed amongst people (Kaufman \& Beghetto, 2009).

This concludes the section of the review which has presented western research conducted in the field of design and creativity education. As was outlined, there is a significant lack of research which connects DT specifically to VCD education. However, the synergies between design and creativity were highlighted by the Lee et al. (2019) study mentioned above as it drew on tenets of creativity theory with a focus on interdisciplinary practices for innovation. 


\section{Asian studies}

Research conducted within China's borders by researchers from the west is limited to three studies, all of which have focussed on addressing sterotypical issues associated with the creativity deficit.

United Kingdom researchers examined the efficacy of creative teaching approaches at the Sichuan Fine Arts Institute. The authors, Dineen and Niu (2008), asserted that their participants found it alarming to accept that creativity required new ways of thinking. The students experienced difficulties in accepting uncertainty and the possibility of failure, and it took time to establish environments of trust and support so confidence and independence could grow. Furthermore, teacher-centred approaches used at the institute typically placed high value on outcomes, and these approaches were found to result in work lacking depth and meaning (Dineen \& Niu, 2008). Torrance tests were used to examine the effectiveness of a ten-week creativity training course, designed to develop creative thinking in freshman (first year) design students at a private college in Shanghai (Zhu, 2015). Zhu's Californian university thesis argued that increases in "creative thinking levels" (p. 95) were evidenced when students were trained in creativity techniques. This research aimed to address the Chinese student creativity deficit and was published in the same year that the 2025 created in China strategy was implemented. In contrast to Zhu's focus on creative thinking within one course, Mullen (2017) provides rich case studies of creative expression in five selected primary and high schools within the Chongqing area. She revealed the paradoxical nature of creativity in some of the schools. Notably, Mullen found creative practices were integrated into curriculum despite the high-stakes accountability within China's test-centric regime. Mullen confirmed her findings with participants at a teacher training institute. She contested the Chinese student creativity deficit by arguing that a creativity paradox exists in China (Mullen, 2017).

The research published in English language journals relating to DT and innovation in Asian countries tends to be confined to imported pedagogical models being used by Asian researchers and teachers. The DT process was used within the curriculum in two Korean studies (Kim \& Lee, 2016; Lee, 2019). Kim and Lee's (2016) qualitative study 
applied a form of DT in a "visual representation of one's life" (p. 206) project in a field trial. A cognitive activity-based design methodology (a form of DT) was pilot tested first by expert designers, and then used by 22 first and second year South Korean VCD students. They followed an adapted DT process checklist (referred to as a methodology) for the design project through a five-hour timeframe. The checklist provided suggestions to aid decision making during the DT phases if any issues arose. Tools and modal shifts of activity through the various process phases were recorded on timesheets by participants. An emphasis was placed on the problem formulation stage as learners negotiated ambiguous design problems. Findings revealed multi-modal, simultaneous and sequential activities, and the authors argued the learning was process-oriented rather than result-oriented, and deeper solution generation developed from the pedagogical approach. The idea of the checklist from this study was adapted for the teaching that took place during my $\mathrm{PhD}$ research, owing to the way in which the design process could be broken down, and the way tools were introduced at various stages of learning.

Another study adopted a project-based learning approach in a Korean Science and Technology Institute which aimed to introduce 130 business administration and science students to sense making processes (Lee, 2019). The study used the English Design Council's double diamond DT model (The Design Council, 2005). In brief, project-based and applied learning is said to encourage skills learning through experiencing real life problems as described by Kolb's (1984) experiential learning model. The rationale behind the double diamond shape presents a process whereby a number of possible creative ideas are generated and opened up through divergent thinking. These ideas are then refined, narrowed down and converged into the best idea. This diamond shaped process happens twice, firstly to identify the problems and secondly to identify the solution. The Design Council asserts that a common mistake is made when the left-hand diamond, the divergent diamond, is omitted and as a result, the wrong problem is solved. Lee (2019) applied this model in the curriculum.

Students were tasked to design products and services in order to revitalise a traditional street market which was suffering decline due to increasing competition from large supermarkets and transitory populations. Six multi-disciplinary teams were instructed on how to "think in design terms" (p. 261) and emerging design technologies and 
software were utilised for the project including 3D printing, website platforms and video editing tools for a documentary. Lee's study identified that the use of DT to generate solutions for problems resulted in positive twenty-first century education influences across disciplines.

Yuan, Song, and He (2018) drew on western theorists for their study that was carried out at a university in Changsha. The study devised a learning by doing strategy for undergraduate industrial design learning. Kolb's (1984) experiential learning model and Schön's (1987) theories were applied, and evolving design learning styles were analysed. This quantitative study was conducted over three years across multiple student cohorts. It began with a pilot study ( 80 participants), progressed to a full study (320 participants), and concluded with a validation study (320 participants). Survey results and statistical data analysis revealed that foundational students prefer Type A learning (from doing to seeing) which means mastering skills from concrete experience. Students working at more advanced or higher levels prefer Type B learning (from seeing to doing), which involves a facilitation of problem-solution evolution and movement towards active experimentation. Of note from this study are conclusions that were drawn about Type A learning:

In this case teachers should act as coaches assisting students in understanding basic practical techniques. For example, teachers can demonstrate an operable process towards a specific design problem and then assign similar tasks to students so that they can experience the procedures on their own. When students encounter difficulties in practice, teachers can provide step-by-step instructions on tools and techniques and present more similar cases and examples. (p. 327)

This except reflects the way in which the students at the university were supported in their acquisition of tools and technical skills learning in order to produce an outcome.

A case study from Nanjing in mainland China described how online collaboration and interactions between six high school teachers and an undisclosed number of students were initiated (Jiang, Tang, Peng, \& Liu, 2018). The authors asserted that high school 
students are disadvantaged by their lack of basic knowledge in innovative creative thinking and practical design skills when preparing for higher design education. The problems the high school students were faced with included strong competition for limited university places and parental perceptions that drawing, and painting skills were of primary importance. According to Jiang et al. (2018), "students [in high schools] have long been educated in a way in which innovation and creativity are encouraged less than memorizing knowledge and applying it in various competitions through which a student can become more recognized" (p. 192). The authors implemented a collaborative web-based framework (a social media application) which integrated aspects of DT within the curriculum design. Students were asked to design a portable stool, and multiple resources such as designs of other stools and manufacturing requirements were uploaded. Decisions about the student work appeared democratic owing to a voting system that was implemented. The authors evaluated the learning through questionnaires, observations and interviews, and they concluded that online sharing was useful because it eliminated arguments as students were more concerned with organising their ideas, language, and efficiency (Jiang et al., 2018).

\section{Transnational higher education (TNHE) studies}

A wide variety of research has been conducted within the TNHE context, and there is a rich policy and practice literature. The literature includes arguments for a common transnational education framework, or classification systems to be established to understand Chinese student movements, and to distinguish between different forms of TNHE (twinning, franchise, joint/double/multiple degree programmes, international brand campuses, cofounded institutions, franchise universities and distance education) (Hu \& Willis, 2017; Knight, 2016; Xu \& Montgomery, 2019). The global reach of TNHE activities with a focus on China has been captured by many in the form of reviews (Caruana \& Montgomery, 2015; Qin \& Te, 2016; Xu \& Montgomery, 2019).

Caruana and Montgomery (2015) reviewed TNHE research published between 2006 and 2014, and argued that the preoccupation with "issues of policy, governance and regulation [was contrasted by the] paucity of research exploring curricula, pedagogy 
and student support mechanisms" (p. 22). Qin and Te (2016) reviewed 550 combined national (mainland China and Hong Kong research) and international articles. Most of the research from these combined sources focused on themes of overviews and trends (73.3\%), and cross-cultural issues (7.2\%) were found only in national literature. This particular literature focussed on "cultural differences, educational sovereignty, core values and ideology, and political and moral education during cooperation” (p. 309). Qin and Te's (2016) study was critical of the national research, suggesting that the quality was low, and that the field is still in a phase of "theoretical maturity and methodological sophistication” (p. 313). Xu and Montgomery's (2019) narrative review identified 250 articles and documents which related to the mobilities of students from China published between 2010 and 2018. The authors outlined three key insights: influences of the Chinese state on mobilities, a requirement for more research to be conducted including longitudinal studies to better understand the fluidity of movements, and a "theoretical and conceptual toolkit" (p. 598) to be developed in order to locate student mobilities.

In the months following my data collection, two pertinent Australian articles were published (Dai, 2018; Dai et al., 2020). Dai (2018) outlined a personal narrative of his experience when transitioning through a TNHE programme; he discusses how agency, belonging and sense of identity required constant adjustment. The 2020 research was situated within an undergraduate two-plus-two transnational programme; students spent two years in Shandong province, China, followed by two years in Queensland (Dai et al., 2020). The context was a digital design programme which is slightly different to VCD, owing to its focus on technical programming language and design for animation, web and other online applications. All the courses in China were delivered by Chinese lecturers, with the occasional visit by an Australian guest lecturer who would deliver a lecture about the Australian context. Semi-structured interviews were used to collect data from a cohort of twelve students who moved to Australia, with findings revealing themes tied to learning, and academic and culture shock. Dai et al., (2020) found that the "limited scaffolding and the indirect teaching approach adopted by Australian lecturers" (p. 8) were navigated successfully by some of the participants, but others struggled. The author called for future research to consider how 
cultural and educational differences may be better understood to ensure improved outcomes for TNHE students.

\section{Conclusion}

Qin and Te's (2016) review in particular revealed two gaps to be addressed within transnational literature. The first relates to teaching and learning within a particular discipline. Research related specifically to learning within TNHE design courses, whilst limited, does exist (Dai, 2018; Dai et al., 2020). However, this research was focussed on TNHE student experience rather than a focus on VCD student engagement with creative design learning. The second gap in the TNHE literature relates to crosscultural creativity issues. Although this was not my original focus, as the research progressed I became interested in understanding Confucian cultural values in relation to creative design process learning.

The scarcity of graphic VCD and/or communication design literature within higher education, not only from western sources but also from Asian researchers, highlighted challenges. Although research used DT (Jiang et al., 2018; Kim \& Lee, 2016; Lee, 2019), DT is not a theoretical framework. Rather, DT is a prescriptive process model which uses specified guidelines to solve design problems; therefore, in practical terms it is a useful pedagogical approach for design learning. As I had adopted DT in the pedagogical design of the projects that I used as case studies, it was important to position the use of DT within other contextual implications, and to appreciate that other pedagogies may exist inside higher education classrooms in China. Wider contextual concerns, such as the political landscape and the influence of Confucianism in Chinese education, required a theoretical framework which may help account for the 'what, why and how' of VCD educational practices. Furthermore, I questioned how social, cultural, and historic forces may have influenced learning within this context. Therefore, the decision was made to frame this $\mathrm{PhD}$ research within a $\mathrm{CHAT}$ framework, to enable the emergence of new epistemology about this particular situation. 


\subsection{Framing the research: the rationale for using cultural historic activity theory}

Creativity and creative design process in education in China exist in continual states of pedagogical flux, particularly when viewed within the historical context (Wang, 1989). Design education is undergoing change processes driven by the politically motivated 2025 strategy, but also by imported practices and understandings of design pedagogy. The potentially confronting teaching practices arising from philosophical and cultural differences may impact the way students engage with creative design process learning (Barratt-Pugh et al., 2018). Invariably, tensions and contradictions arise from differing social, cultural and institutional relationships when imported approaches are included in teaching and learning in China.

In consideration of the predominance of change and flux in creative design education in China I chose the analytical framework of CHAT to inform both data generation and data analysis. Three core ideas, discussed in more detail in the next chapter, are fundamental to understanding a CHAT analysis: first, developmental change in humans as they learn by doing, second, the role of tools and labour in transforming relationships between humans and environment, and third, that social experience within a community is essential to the "process of making and interpreting meaning and thus to all forms of developmental learning, communicating, and acting" (Foot, 2014 , p. 3). Theoretical frameworks of CHAT research have evolved through three generations, with the most recent version, the third generation, reconceptualised as activity theory (Engeström, 2001, p. 133). Engeström's interpretation of AT which is based on a collective perspectives, proposed a number of AT principles in addition to the core ideas mentioned above, including: "multi-voicedness" in which multiple participant voices may become a source of innovation, and "historicity" which has the potential to shed light on problems against their own history (Engeström, 2001, p. 136). The affordances of CHAT with its analytical AS approach means an emerging story may be depicted as a model, and the myriad of relationships within single or multiple activity systems may be articulated (Foot 2014).

The small number of researchers who have used CHAT within the VCD area, have mostly used second generation CHAT. Vygotsky (1978) proposed an individual 
orientation of actions and the effect of this on group activities within a single activity system. For example, a case study examined the way in which professional graphic designers focused on the interplay of subject, tool, object interaction within the conceptual development phase (Tan \& Melles, 2010). Similarly, in a graphic design and information communication and technology study in South Africa, the ideation phase was examined through a widened interplay of activity system component parts including rules, community, and division of labour (Appiah \& Cronjé, 2013).

Interestingly, CHAT was used in a recent case study investigating the effect of educational reform on pedagogy in China (Barratt-Pugh, et. al., 2018). The participants were 66 mainland Chinese academics from 49 different universities from across China on a 13-week Australian study tour. Participants were aged between 26 and 45, all from business disciplines with 1-7 years' teaching experience. Grounded theory was used and in total, 63 coded categories were linked to the activity system components in order to construct a comparative map of issues, experiences, and reflections expressed by the participants. Findings revealed that "regulated knowledge sources, knowledge dissemination, student compliance, parental expectations, examination achievement and pastoral care [were the driving concerns, therefore] traditional practice, social expectations, local regulations and economic restraints" were frustrating reforming practices (Barratt-Pugh et al., 2018, p. 831). The authors assert that this study was important as it was the first to explore these issues with activity theory, and the research has implications for restructure and reform from within China.

\subsection{Research questions}

The main research question guided the bodies of literature I reviewed, and two sub-questions were conceived as I undertook my data collection and have been subsequently justified by the review. These questions are now presented.

\section{Main research question:}

How do VCD students at a university in China engage with creative design process learning? 


\section{Two sub-questions:}

1. How do previous and current Chinese teaching and learning practices shape VCD creative design process learning?

2. How do imported and unfamiliar educational practices influence VCD learning activities?

In the construction of the research questions, there was a requirement to acknowledge the debate about learnification in education (Biesta, 2012). This in itself suggested a particular terminology be used for the research questions, and indeed, for the thesis. Biesta (2012) discussed dichotomies in the "rise of a new language of learning" (p. 37) as a result of post-modern and neo-liberal critique. In this critique, "learnification" means the learner is placed at the centre of their learning, and the role of the teacher is reduced to focusing on ways in which to support and facilitate learning (p. 37). However, unlike a previous focus on transmission of content from teacher to student in the so called "instruction paradigm" (p. 36), Biesta asserts that in contemporary education, students learn something, from someone, for a particular purpose. This “explicitly progressive” (p. 36) debate argues crucial educational questions about "content, purpose and relationships" (p. 36) of teaching practice, and it is here that synergies are aligned. By synergies, I mean similarities are found between Confucian education which believes the purpose of learning is progressive (hierarchical) and linked to relationships, and how Biesta's perspective asserts the purpose of education is to set a direction of learning. Both perspectives look to teachers to identify pathways in education to achieve higher knowledge. Therefore, setting a direction in learning is based on judgements and decisions about content which should be sensitively adjusted for uniquely different contexts (Biesta, 2012).

The choice of the term "engage" within the main research question also required consideration of a number of points. Highly influential in higher education, engagement is connected to learnification (Gourlay, 2017). Engagement is referred to as a "good thing" (p. 23) in which overt displays of practice, activities and performance are encouraged to optimise student experience and learning outcomes (Gourlay, 2017). Gourlay argues the term to be weakly theorised as it does not express what engagement consists of in terms of practice, when it is so heavily influenced by 
active and demonstrative collaborative practices. The main issue, or tension here, is that the mainstream policy and practice emphasis on social and collective learning is that it does not account for individual performance (Gourlay, 2017). This argument indicates a slight shift from a fundamentally humanist conception which places agency in human hands within teacher-student relationships to an idea whereby non-human actors, including context and material tools, for example computers, enable agency and engagement. Gourlay calls it a recognition of the "socio-material and radically distributed nature of human and non-human agency [in which] richer and more nuanced range of ways" (p. 33) of day-to day student practices might help conceptualise student engagement. There are connections between these ideas and CHAT, in that both are considerate of social, cultural, historic contexts, and the term engagement can account for student interaction with materiality and tools as well as collaborative and quiet individual interactions.

The two sub-questions were conceived as I undertook my data collection.

The first sub-question, how do previous and current Chinese educational practices shape VCD creative design process learning, was further justified after reading about step-by-step teaching and learning in the Yuan et al. (2018) study in Changsha. This was unfamiliar territory, and I became curious to learn how local pedagogical practices may enable or constrain learning. Furthermore, as the research journey progressed, it became ever more critical to understand how previous teaching and learning practices had influenced creative design activities of the HNCU students, which in turn referenced Engeström's (2001) third AT principle "historicity” (p. 136).

The second sub-question, how do imported and unfamiliar educational teaching practices influence VCD learning activities, was shaped by my curiosity to learn about how the VCD students navigated and made use of the new practices? In addition to this, the question references Keane (2006) who asserted western ontological perspectives about creativity and creative design processes, and their association with freedom and liberal democracies are unlikely to work in China. As a New Zealand educator and researcher, Kean's assertion had the potential to directly challenge my teaching practices and perceptions about the VCD learners at HNCU. Furthermore, my 
interpretation of DT as a pedagogical approach in the classroom, left little room for other learning approaches, for example, Chinese repetition, or Confucian philosophical underpinnings to be accounted for. To counter this, Engeström's (2001) activity theory principle, "multi-voicedness" (p. 136), pointed to the notion that multiple participant voices should be sensitively navigated to allow for creativity and innovation to emerge. Therefore, this sub-question aimed to draw conclusions about my dual role, that of teacher and researcher as a potential influencer on VCD learning activities.

To conclude, the questions this thesis proposes to answer have clearly not been addressed to date nor has a sustained CHAT analysis been conducted of teaching and learning in the field of VCD within a transnational context. A detailed explanation about CHAT and the role it played in this research follows in the next chapter. 


\section{Chapter 3. Theoretical and analytical framework}

This chapter begins with a recap of the three core ideas that help make sense of Cultural Historic Activity Theory (CHAT). These are: developmental change in humans as they learn by doing; the role of tools and labour in transforming relationships between humans and the environment; and the role of social and community experience in making and interpreting meaning, sometimes referred to in some of the literature as sense making (Foot, 2014). These ideas of CHAT and Foot's explanation about the significance of each of its key concepts points to the benefits of employing this theoretical and conceptual framework. Each term is explained in turn. Culture signals the idea that the activities that occurred within the VCD classroom at HNCU were located within a particular cultural context. Within this educational context, historic refers to the idea that the students' actions and activities were located within historic trajectories of learning. Activity, or the doing, referred to the combined or collaborative classroom practices, or in other words, the "situatedness" of learner activities that were modified by cultural and historic influences (Foot, 2014, p. 3). When these three theoretical ideas are combined, it can be argued that culture is integral to learning, which means what we learn and how we learn is based on historic learning experience constructed through community shaped values, beliefs, and ideas (Nasir, Rosebery, Warren, \& Lee, 2005).

\subsection{The evolution of cultural historic activity theory (CHAT)}

There are two methods to observe cultural learning that relate to a particular community. These are behavioural sign and signification of human interactions, and through engagement with social networks (Van Der Veer, 2007). There are some close links with these two methods to the ideas of CHAT formulated in the early nineteenth century by two Russian cultural psychologists, L. S. Vygotsky and A. N. Leont'ev (please note: Leont'ev is translated variously as Leontyev and Leontiev which is reflected as the authors intended in the in-text citations). The following section explains their ideas, then introduces the further evolving frameworks categorised as three generations of CHAT. 


\subsection{The CHAT generations}

The historical roots of CHAT are traceable in the writings of Marx and Engles, but it was Vygotsky's writings that demonstrated opposition to mainstream psychological thinking in the 1920s and 1930s which contested that animal and environment were intertwined entities (Engeström \& Miettinen, 1999). Putting this into context, Leontyev (2009) explains positivists view the environment as something humans, like animals should adapt to for survival. This differs to the view that understands environment, which includes society and culture, as something that produces human activities. Epistemological beliefs of early cultural-historic activity theorists posited that humans adapt their activities to external conditions, but also that these conditions include social conditions which in themselves carry the "motives and aims of [an] activity" and "that society produces human activity" (Leontyev, 2009, p. 397).

First generation CHAT stems from the contributions made by Vygotsky (1978), who proposed a changing "dialectical and historical materialism" of phenomena (p. 6). He suggested a belief that individuals internalise culturally produced sign systems in childhood, and later forms of development and behavioural transformations are "mediated" by tools in "human-environment" interactions (p. 7). Therefore, the power of tools to extend human activity stems from the users' cultural-historical origin, which incorporates expanding knowledge from one generation to the next, thus becoming a means of expression for a particular culture (Leontyev, 1981). Cultural learning phenomena can be determined as fluid, societies that create and grow their own tool (specialisation of the hand in mastering nature) and sign systems over time. An example of this Vygotskian tool/sign systems concept in the field of VCD learning is found in the relationship between a pencil (tool) and sign (language). When a pencil is used in the action or activities, such as in drawing, idea generation or rendering by hand, certain behavioural responses become evident that can be identified as being specific to the VCD classroom community. The sketches or drawings produced by learners are likely to reflect previous demonstrations, meaning the way they were taught through language how to sketch or draw, remains residual. In much the same way, language is learnt in response to signification, which in turn means that humans 
learn from "certain stimuli" the sign provokes, and this in turn mediates a return human response (Van Der Veer, 2007, p. 28).

Vygotsky's (1978) contribution to CHAT theorised this mediating role of social interaction on developmental learning. His triangular model illustrated how stimulus and response was mediated by an "auxiliary" complex, mediated act (p. 40) (see Figure $3.1 \mathrm{~A}$ ). Within contemporary articulations of CHAT, stimulus and response are now referred to as a subject and an object, an auxiliary complex act defined as mediating artefact, and Vygotsky's model has been reinterpreted see (Figure 3.1 B).
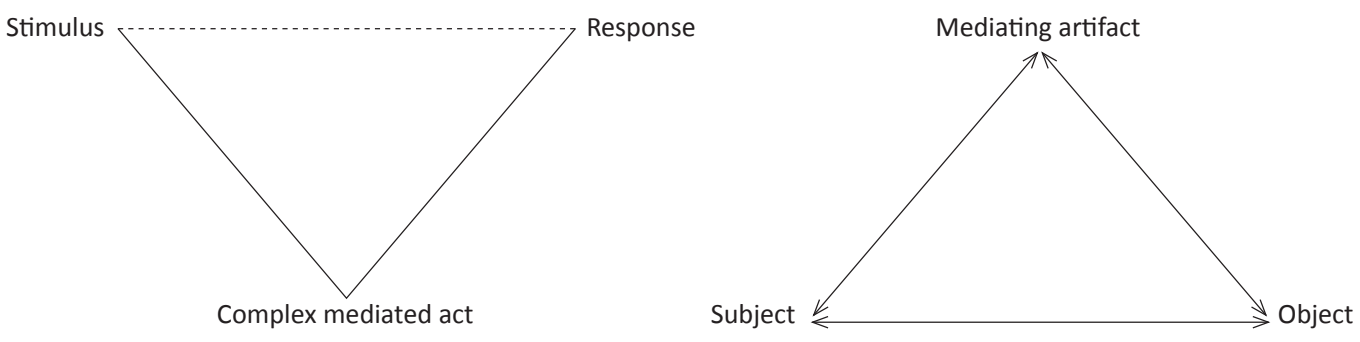

Figure 3.1 (A). Vygotsky's model of a complex mediated act and $(B)$ the common reinterpretation of a mediated act (Engeström, 2001, p. 134).

This early model outlined the first three named components of an activity system (AS): subject, object and mediating artefact. One of the problems with Vygotsky's culturalhistorical school ideas which recognised cultural tool and sign mediation is that they did not take into account other mediating influences such as the richness of human interaction, social relations, or social networks (Engeström \& Miettinen, 1999; Van Der Veer, 2007). Despite this, and the changing political climate in the Soviet Union from the late 1920s in which Vygotsky's theories on human intelligence and consciousness were suppressed after his early death in 1934, Vygotsky's legacy and spheres of international and multidisciplinary influence increased in relevance for scholars during the 1980s and 1990s (Daniels, Cole, \& Wertsch, 2007; Engeström \& Miettinen, 1999).

Soviet cultural psychologists Leont'ev and Luria advanced Vygotsky's theories after observing workers interacting with "certain environments," and they questioned "how the development or design of specific tools helped workers to satisfy certain needs or 
goals" (Disarro, 2014, p. 439). Specifically, Leont'ev, made somewhat of a conceptual jump in the theory after a careful reading of Marx (Engeström \& Miettinen, 1999).

Second generation CHAT developed after Leont'ev recognised that within the division of labour there were distinctions to be made between individual and collective activities and actions. Two important descriptions and explanations about this distinction helped guide aspects of my research. The first distinction from Leontiev (2005) himself describes the processes in play when a person maintains fuelling a fire. He questioned the purpose and focus of maintaining a fire, and argued linkages between the individual activity of maintaining a fire for nourishment and collective group activities of hunting animals to cook on the fire. He talks about the object of the fire, stimulating both activities of the fire maintainer and the hunters as having a special nature related to co-existence or similarity. In other words when both activities are united and each party plays a role in the process and purpose of fire, hunger is relieved. Engeström and Miettinen (1999) discuss Leont'ev's description of a primeval beater taking part in a collective group hunt in a similar way. However, when the object and motive of the individual beater, and that of the collective group differed or did not coincide, then the distinction between activity and action is made. Leont'ev called this mis-alignment of object or motive, an action in his example of a student preparing for an examination; mastery of the books content is not its motive, is not what induced the student to read it, but rather "the need to pass the exam" (Leontyev, 1981, p. 400). Taking this one step further, Kaptelinin (2005) argued that Leontiev's metaphoric examples, which illustrated dissassociations between activities and actions, may be better understood as dissassociations between motives and goals which emerged as the result of the division of labour. Thus, the conceptualisation of a threelevel model of activity theory was characterised by Leont'ev's division of labour (Engeström \& Miettinen, 1999). The top level of collective activity is "driven by an object-oriented motive; the middle level of individual (or group) action is driven by a goal" and the bottom automatic operational level is guided by a set of "conditions and tools of action at hand" (Engeström \& Miettinen, 1999, p. 4). 
Leont'ev never depicted second generation theory in a model; however, Engeström (1993, 2001) further characterised and depicted a compartmentalised activity system (see Figure $3.2 \mathrm{~A}$ ).
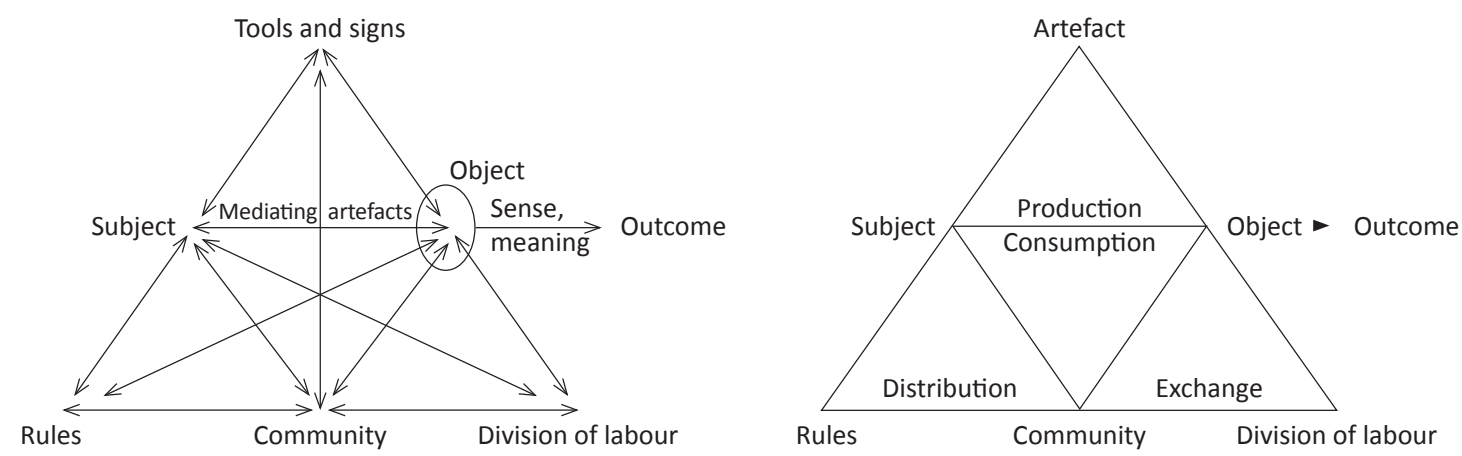

Figure 3.2 (A). Second generation CHAT, from the basic structure of a human activity system, and (B) production and consumption of an activity system (Engeström, 1993, p. 68; Engeström, 2001, p. 134).

Human actions and behaviour are analysed, and goals, motivations, and interactions of a collective group may become understood in relation to influences of social, cultural and historic precedents (Disarro 2014). Furthermore, Engeström's representation described the relationship between the components of an activity system (Roth, 2009): production of an outcome by a subject is object oriented, distribution of objects' reciprocal interaction and exchange for individual needs is divided between a community, and the end result is consumption (see Figure 3.2 B). Consumption as a product is said to step outside this social movement (Bourke, Mentis, \& O'Neill, 2013; Roth, 2009).

Thus, the theoretical focus shifted towards viewing human activity as the unit of analysis, rather than psychological internal cognitive processes being the focus (Leontyev, 2009). In other words, nowadays it is generally acknowledged that internal thought processes are "produced from external" social, cultural and historic sources (Leontyev, 2009, p. 399). Vygotsky's theoretical contribution focused attention on a unit of analysis whereby "object-oriented action [is/was] mediated by cultural tools and signs" (Engeström \& Miettinen, 1999, p. 4). But followers of Leont'ev tend to focus on collective human activity as the basic unit of analysis based on Leont'ev's distinctions between an individual's activity that does or does not necessarily coincide with collective activities of a group (Engeström \& Miettinen, 1999; Leontyev, 1981). 
Significant challenges are noted with applications of second generation CHAT, which relate to diversity and dialogue between different traditions or cultural perspectives; therefore third generation CHAT emerged to deal with these issues (Engeström 2001).

Third generation CHAT, introduced by Engeström, was a further step in the historical development of CHAT. Third generation CHAT, referred to interchangeably as either CHAT or activity theory (AT), expanded to include a minimum of two interacting activity systems (see Figure 3.3).

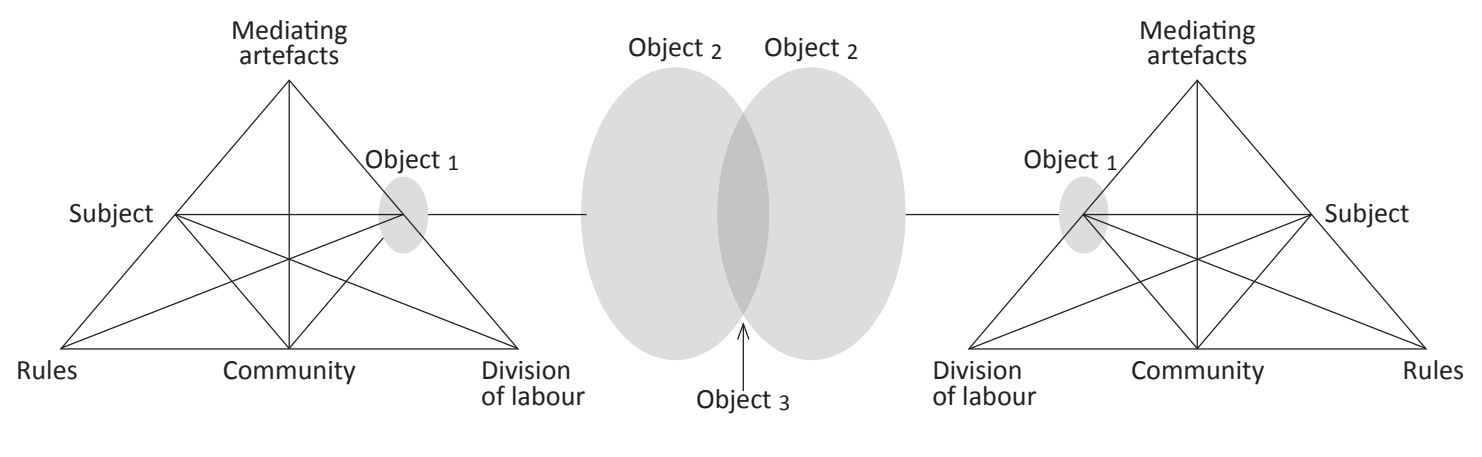

Figure 3.3. Third generation activity theory, two interacting systems as a minimal model (Engeström, 2001, p. 136).

This model moves the subject from being a singular entity to that of an entity which may become understood through collaboratively constructed viewpoints. This changing CHAT perspective gives rise to the differences and interpretations about objects between Leontiev and Engeström, which have been subject to debate owing to problems with translations (Kaptelinin, 2005). Although Leontiev's ideas did take into account social and cultural practices, values and tools within collective activities, Kaptelinin (2005) highlighted that Leontiev's analysis primarily dealt with "activities taking place at an individual level” (p. 9). Leontyev's (2009) perspective, formed within the context of psychology and defined by the division of labour, proposed:

The object of the activity gives it a determined direction ... [and] the object of an activity is its true motive. It is understood that the motive may be either material or ideal, either present in perception or exclusively in the imagination or in thought. (p. 98) 
Engeström amplified this perspective by exploring the complexity of interactions and relationships in wider social, cultural and historic contexts in his approach to an activity system unit of analysis. The idea of community interaction was introduced whereby the unit of analysis is conceptually defined as "object-oriented, collective, and culturally mediated human activity or activity systems" (Engeström \& Miettinen, 1999, p. 9). Thus, the object of activity in third generation CHAT is defined as a problem space containing three facets which were illustrated by health vignettes: first, an object is "a thing-to-be-acted-upon" (the illness), second the object is questioned (search for a cure), and third a desired outcome is reached (the cure) (Foot, 2014, p. 333). For Engeström (2001, 2005), activity networks, boundary-crossing and expansive learning are being articulated through expanded objects. The theory of expansive learning is "based on the dialectics of ascending from the abstract to the concrete" (Engeström, 1999a, p. 382). This means that inner contradictions which may be traced as historic problems are resolved, and as students grasp the essence of their object (the motive), their abstract ideas are transformed and "enriched [in] step by step ... constantly developing manifestations" (Engeström, 1999a, p. 382). This concept contradicts traditional learning situations where competent teachers teach skills that lead to changes in individual learning behaviour. The main premise is that learning is unstable, learning is learnt as it is being created, and as a result the object (motive) is constantly moving, and short-term goals or outcomes may not be realised (Engeström, 2001). An example of this may be seen in interactions between the teacher and learner where motivations differ, and this overlap forms new and unexpected revelations, thus moving into a new form of transformed production (Engeström, 2001).

Like second generation CHAT, third generation AT uses the Activity System (AS) core components: subject, object and outcome of the activity, tools or mediating artefacts, and at the base level of the triangle the rules, community and division of labour are depicted (Engeström, 1999). Both CHAT/AT and an AS analysis are argued as being beneficial for research into learning owing to their dynamic and non-static features. Inherently dialectic, all of these features include first, the subject/objectoriented unit of analysis, and second human praxis, both synonymous with change and exchange processes (Roth, 2009). Furthermore, learning activities and actions produce individual and collective outcomes. Thus, for the individual learner whose goals are 
aligned, or mis-aligned, with the object, means of production, social and cultural interactions (rules, community, division of labour), a form of transformative reproduction takes place (Roth, 2009). Lave (1993) describes transformative reproduction or change in knowledge and action as synonymous with participation in learning activities. To illustrate in brief with an example of transformative reproduction from my research context, an individual VCD learner produces design work for distribution, exchange and consumption by a learning community, whilst simultaneously this same process reproduces an individual VCD learner as a member of that community (Roth, 2009).

Engeström (2001) proposed a framework of five principles to apply in third generation AT which helped guide my research. The first principle suggests that a unit of analysis should be a collective, artefact-mediated and an object-oriented activity system, should be interpreted against a background of entire or other activity systems. The second principle revolves around "multi-voicedness" as a source of innovation, but the researcher should sensitively navigate multiple participant voices. The third principle referred to as "historicity" has the potential to shed light on problems against their own history, for example, examining previous ways of doing that were both taught and learnt. The fourth principle focusses on contradictions as sources of change and development, due to activities being open systems, for example contradictions occur when the system adopts a new element, such as technology, and rules or divisions of labour collide owing to the disruption. Finally, the fifth principle "proclaims the possibility of expansive transformation (p. 136)." In other words, the fifth principle suggests that ASs may become re-envisioned, and re-conceptualised owing to the fluid nature of social, cultural and historic contexts. Potential problems that are raised from the developmental perspectives in these principles are that often historic aspects are missing from AT research (Moran \& John-Steiner, 2003).

Finally, within a conceptualisation of expansive learning in third generation AT, Engeström (2005) suggested that in putting forward any theory of learning - that theory needs to be able to answer four fundamental questions: 
(1) Who are the subjects of learning, how are they defined and located?

(2) Why do they learn, what makes them make the effort?

(3) What do they learn, what are the contents and outcomes of learning

(4) How do they learn, what are the key actions of processes of learning? (p. 59)

Using these questions in research involves analysis of changing object motives, and praxis and may point to possible transformative learning capabilities (Barratt-Pugh et al., 2018). Barratt-Pugh et al. (2018) explain in a particularly useful way that the benefits of using these questions are due to the way pedagogic change is approached from a holistic perspective, and that equilibrium may be regained between the "goals, roles, materials, power, social objectives and institutional norms associated with pedagogic practices" (p. 832). But, for the impact of change to be balanced, first a deep understanding and explanation of the tensions and contradictions must be elaborated. To this end, CHAT recognises the critical role of social and cultural historic processes in activity processes, and enables complex multi-voiced pictures of people's interactions, social and cultural forces, and historical time in motion to be presented (Engeström, 2001).

\subsection{Activity system (AS) components}

A key revelation in conducting CHAT analysis at a particular point in time is that in order to grasp the systemic activity as a whole, it is important to understand that an analysis should not centre on any of the six AS components alone (Foot, 2014). There are six core components - subject, object, artefact, rules, community and division of labour - that comprise an AS, and these components are commonly depicted as the nodes of an AS, as previously seen in the CHAT generation figures (see Figures 3.2 and 3.3) (Vygotsky, 1978).

The subject of an activity system may consist of an individual or a group (Disarro 2014). In this $\mathrm{PhD}$ research the subject consisted of dyads (two learners working together) situated in the context of an undergraduate VCD classroom in China. The focus on pairs of learners was decided in direct response to Foot (2014) who discussed the analytical strength of CHAT being leveraged when collective object- 
orientated activity is examined within an AS. Foot's research exemplified the AS through the object-orientated activity of doctor and patient, whereas in this study two learners of similar age and experience were placed together. The social and cultural collaborative nature of design thinking processes that underpinned the overall pedagogical approach of the teaching provided further justification for the decision of analysing dyads. Furthermore, in order to enrich meaning, an examination of dyads in conjunction with the other influencing AS components held the potential to highlight individual and collective group actions and activities in the tradition of Leontyev (1981). The application of the theoretical framework in this way formed the basis for the analysis, as the overall aim was to elicit understanding about Chinese VCD learners' engagement with creative design processes. It was hoped than an awareness of engagement may help influence personal directions of future teaching and learning activities. However, as each participant was unique, their engagement with the activity and motives towards participating in a design process were different, limiting any kind of generalised conjecture for future teaching (Disarro 2014).

In introducing object, it should be clearly articulated that from an activity theory perspective, object-orientated activity is the unit of analysis (Yamagata-Lynch, 2010). To explain this, the object of an activity system is the "problem space" and is therefore associated with motivation or objectives that the subjects direct towards an activity (Engeström, 1993, p. 67). An AS is therefore centred on the object, which aids understanding about object-orientated activity being viewed as the unit of analysis. Objects, should not be confused with the goal, outcomes; "the motive, separate from the goal, comes at a certain stage in the development of the given process toward to goal itself" (Leontiev, 2005, p. 64). Moreover, the concept of motive correlates with the concept of activity, whereas the concept of a goal correlates with that of an action (Leontyev, 2009). Ideally, learner engagement with an action or activity is foreshadowed by a teacher's deep awareness of the types of motives that are in play in the classroom (Disarro 2014). As a teacher, my motives for the two particular VCD pedagogical projects examined in this research related to unlocking strategies and techniques in order to transform creative design process learning, and the purpose related to embedding design thinking process learning. Motives and goals of the learners are discussed in various places in the finding's chapters. 
Mediating artefacts broadly speaking can be anything that enables or scaffolds student activities (O’Brien, Varga-Atkins, Umoquit, \& Tso, 2012). These particular AS components are discussed in the following section.

\subsection{Analytical framework}

\section{An AT methodological contribution for creative design process research}

A number of issues arose for me as I grappled with the interview transcripts for the analysis. I found it difficult to understand how artefacts related to creative design process learning. Primarily, my problems related to the way an artefact or toolmediated activity, a key concept of the cultural-historic school and 'context,' a key cultural psychology concept was articulated in the literature (Lave, 1993).

To reconcile this, it was necessary to understand that western discussions about context take various forms. Determinist environmental perspectives tend to exclude the relationship between people's activities and the setting or environment; cognitivist perspectives tend to exclude the societal and cultural aspects of activities, and phenomenological or ethno-methodological discussions focus on individual or experiential perspectives (Engeström, 1993). From an activity theory point of view, the notion of context is more holistic. Contexts are "neither containers nor situationally created experiential spaces;" rather, contexts are integrated subject, object, and artefact unified wholes (Engeström, 1993, p. 67). Thus, a central tenet of the cultural-historic school is that an artefact is a completely integrated component of an AS, and an AS in itself is a context (Engeström, 1993).

Therefore, an artefact may be described as a material object modified by people to regulate or mediate their interactions within the context of each other and the world (Cole, 1999); and, embedded within the materiality of an artefact are symbolic meanings and associations constructed over time within "socially situated activity" contexts (Lave, 1993, p. 4). Humans adopt symbolic resourses that artefacts embody (Cole, 1999); while these are interpreted as part of material culture it is much less evident how the perspectives of the cultural-historical school might usefully apply to creative design process learning within VCD education. Here intangible things, such as 
the arbitrariness of language, imagination, behaviour and visual design processes emerged as crucial concerns. These were especially heightened when working across cultures. Translation and interpretation between different languages inside the VCD classrooms at HNCU had an impact upon the effectiveness of cultural exchanges between me, the interpreters and the students. It was necessary to be sensitive towards the diversity of shared or misunderstood cultural values (Nasir et al., 2005).

Another issue that arose for me during the analysis was that artefacts and tools often appeared interchangeable in the literature. This led to a fundamental question: What is an artefact, and when is it a tool? Vygotsky (1978) distinguished between two types of tools (Hardman \& Amory, 2015) object-oriented external technical tools (tangible tools, for example, a computer) and subject-oriented internal, psychological tools or signs (interpretation and enactment of internal dimensions). Vygotsky's distinction attributed their usage to being divergent, meaning their mediated function worked as a catalyst for different actions (p. 54). Engeström (1999) challenged the distinction, arguing against ideas of internal processes operating "objectively first and foremost as a process" (p. 21). Consequently, the concept of artefacts being employed simultaneously is largely accepted. Thus, everything may come together and be incorporated into an "ensemble of social, material and historical forces" (Hilppö, Lipponen, Kumpulainen, \& Rajala, 2017, p. 361). The issue with simultaneous deployment of artefacts is that distinctions are not made and nothing is explained (Roth, 2007). Foot's (2014) description exacerbates the issue. She describes artefacts as tangled material or conceptual tools "shaped by the needs, values, and norms of the culture(s) in which they are created and used" (p. 331). The problem with these perspectives is that if there was a distinction to be made between artefacts and tools; it was still unclear to me.

The answer lies in thinking about an "artefact as tool" (Wartofsky, 1979, p. 201). Wartofsky's explanation describes an artefact as anything that is created by humans for the purpose of successful production and reproduction. Hence, artefacts carry successful adaptations from earlier times that combine ideal and material culture, and are adopted for the symbolic resourses they embody (Cole, 1999). Along these lines, consideration of an artefact as a tool means an artefact may extend or mediate the 
aquisition and transmission of human skills for a process of production (Wartofsky 1979). Thus, an 'artefact as tool' means the terminology may be used interchangebly because the artefact is deployed as a tool. The important point about mediating artefact deployment in creative learning is that the tools continuously transform production and reproduction. Therefore, cultural motivations or objectives brought to the objects may influence and hold consequence for the dyadic activities (Engeström 2001).

Further conceptual understanding about artefacts evolved as the data analysis proceeded. A particular lightbulb moment occurred as attempts were made to try to make sense of the data that had been collected from the case studies. A description proposed by Cole (1999) and Wartofsky (1979) about a three-level hierarchy of artefacts helped to disentangle artefact mediation specifically for my research. Further explanations from Foot (2014) about secondary artefacts, and Wallas (1926) about design process helped round out my understanding and for all intents and purposes, the three-level approach made categorisation of seemingly disparate activities into coherent themes more manageable. By disentangling artefacts and defining the role of artefact mediation in developmental learning, a more insightful picture was revealed. This is where a CHAT framework developed for the research, with analytical tools tailored for the specific Chinese classroom, offers significant new insight for VCD education, and by extension, a broader literacy for this particular context.

For the analysis chapters that follow, my partly adopted and adapted construction of descriptions for a three-level hierarchy of artefacts was used (see Table 3.1). 
Table 3.1. A three-level hierarchy of artefacts. Constructed by the author drawing on Cole (1999); Foot (2014); Wallas (1926); Wartofsky (1979).

PRIMARY ARTEFACTS. Used unconsciously in basic operations and visual communication design production

Traditional: Paint brushes, pencils

Technological: Computer mouse, computer software (Adobe Creative Cloud), mobile phones

SECONDARY ARTEFACTS. Practical and/or discursively constructed action/activities

Praxis: $\quad$ Hand using a pencil or a computer mouse

Discursive: Objective, subjective and emotional factors which influence deployment of primary artefacts

TERTIARY ARTEFACTS. Processes of the mind

Process: Involving creative aspects (preparation, incubation, illumination and verification)

Abstractions: Involving possible or imaginary worlds

Following Wartofsky (1979), Cole describes the first level as primary artefacts used directly in production, for example paint brushes, pencils, and computers. Primary artefacts are said to be used unconsciously for basic operations (Foot, 2014).

Secondary artefacts are described in two ways, both involving primary artefacts. First, a secondary artefact is simply used as a mode of transmitting action, for example a hand using a computer mouse (Cole 1999). Secondly, and in highlighting the fluidity of objects, discursive constructs are found in secondary artefacts (Foot 2014). Here, Foot provides examples from social workers in which "professionalism, expertise, and objectivity may function as secondary artefacts that influence deployment of primary tools" (336). The mention of professionalism and expertise aligns with design education constructs, and objectivity described as a secondary artefact gave rise to the question of subjectivity and emotion in design pedagogy. For the purposes of an analysis in VCD education it is plausible that subjectivity around numerous design decisions, for example colour choice, may influence how a VCD student employs a primary artefact. Additionally, deployment of primary artefacts may depend on what kind of day the student is having. For example, if a student is happy, he or she may be better disposed towards experimenting with a range of primary artefacts. Conversely, tiredness may result in lack of motivation and consequently lack of object engagement. 
Furthermore, Leontyev (1981) links psychological features of activity containing "special class of psychic experiences - emotions and feelings" (p. 400). He posits the notion of experience to be governed by the object and outcome of the activity through his examples of either walking happily in the rain, or being inwardly numb in fine weather (Leontyev, 1981).

Tertiary artefacts such as imagination, potentially act as tools for changing praxis as they "colour the way we see the actual world" and may be applied to the process of perception (Cole 1999, p. 91). Wartofsky (1979) suggests this is not about dreams, but rather, how the "activity of imagination" is expressed or "embodied in actual artefacts" (p. 209). Put simply, Vygotsky (2004) conceived of drawing (materiality) as a way to master a new culturally significant language (a language of symbolic images) which expands horizons and communicates something that would not be conscious otherwise. Essentially, what Vygotsky was referring to relates to semiotic theory which, in brief, accounted for signs and symbols, for example, an idea in the mind being made tangible in the environment for a community to interpret. Yamagata-Lynch (2010) called this a co-evolving enactment process or practice. Key to these ideas are that tertiary artefacts in the current study acknowledge that notions of imagination, perception and creative process aspects such as incubation and illumination are considered as artefacts in use (Wallas, 1926).

\section{Analytical approach}

Once the distinctions about the three-level hierarchy of artefacts had been made, an analytical approach was devised. Leont'ev's conceptualisation of a three-level model of activity theory characterised by the division of labour was drawn upon (Engeström $\&$ Miettinen, 1999). To recap, object-oriented motives (the unit of analysis) drive the top level of collective activity. The middle level of "individual (or group) action is driven by goal, and the bottom level of automatic operations is driven by the conditions and tools of action at hand" (Engeström \& Miettinen, 1999, p. 4). This distinction was applied in the analytical approach which linked Leont'ev's concept of the three level model with Foot's (2014) "levels of contradictions and corresponding learning actions" (p. 342) and Engeström's (1993) ideas about activity systems being 
“characterized by inner contradictions" (p.74). It was also important to account for Engeström's ideas about object-oriented motives expanding and including collective social and cultural contexts, for example, socio-economic circumstances of students. This is because the third generation of CHAT expands units of analysis towards complex interacting systems, and so diversity and dialogue between different traditions or perspectives may be accounted for (Engeström, 2001; Engeström \& Glăveanu, 2012). A model was employed for the analysis (see Figure 3.4). It should be noted that the top, middle and base levels of this model were contingent on overlapping layers which move up, down and through the activity system, and the tensions and contradictions indicate a "misfit" between the elements of an activity system which in turn has the ability to provoke learning action(s) (Foot, 2014, p. 339).

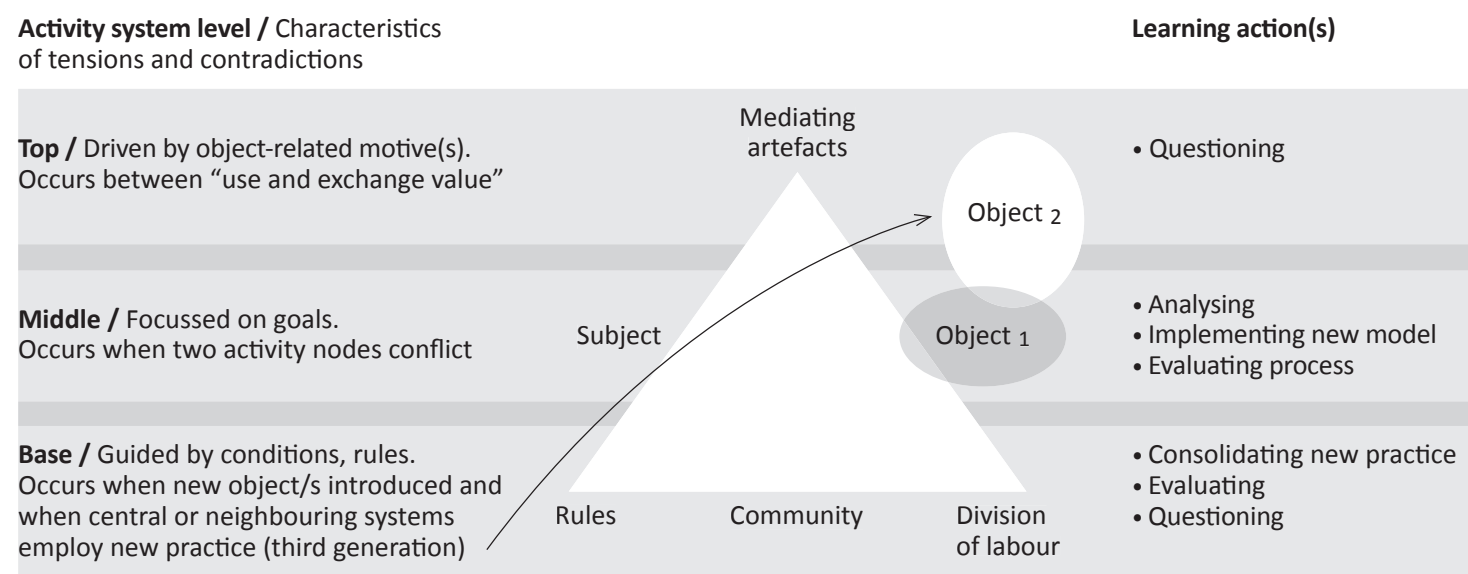

Figure 3.4. Analytical approach (Engeström 1993, p. 72; Foot 2014, p. 339-340).

At the top level of an AS, tensions and contradictions are those which arise from use and exchange value (directly benefits participant and worth something during exchange), for example, the mediating roles of imagination, a tertiary artefact and a pencil; a primary artefact may result in a sketch that expresses an imaginative concept. At the middle level, tensions may arise when two activity nodes are in conflict with each other, such as rules and tools. For example, pedagogical rules may stipulate large numbers of quick 'rough' sketches be made in the design thinking ideation phase. This may become contradicted by a student whose individual action to complete a 'polished' drawing does not align with the group activities. Finally, Foot's (2014) third and fourth levels were combined into the base level of the AS. Here tensions and contradictions may arise when a new, or novel object is introduced into the system to 
provide relief from one or more secondary contradictions and thus, a new developmental phase or innovation may be triggered, and/or a central activity and its neighbouring activity systems may employ a new practice based on the expanded object. An example of this could include something like a new media technique being introduced to generate ideas, and the approach is perceived to be beneficial by the wider group and so activities of all participants become aligned, and limitations of imagination are relieved (Leontiev, 2005). Furthermore, discussion of an expanded object points to the articulation of expansive learning previously discussed in third generation activity theory (Engeström 2001, 2005).

The subject, object, and mediating artefact analysis are located at the top level of an AS and are kept in constant tension, whereas the components in the middle and base AS levels form an expanded sequence which helps explain cyclical learning development (Foot, 2014). To explain the way an expanded sequence works, a subject is potentially influenced or constrained by rules or norms which derive from tacit or explicit social situations governing the activity. Intertwined with rules is the community within and for which the activity occurs, and the division of labour in which the activity is conducted (Bourke et al., 2013; Engeström, 1999).

\section{Theory of creative activity}

Tikhomirov (1999) presented a very convincing argument for a theory of creativity (previously discussed in the literature review) which helped round out the analytical approach. He made the argument that creativity activities are different to routine activities. The differences relate to the orientation of the psyche whereby actions have complete or incomplete orientation bases. By this the author means that in solving complex problems, or operating in so called creative problem/solution spaces, the only possible way to "act is on an incomplete orientation basis" (Dorst \& Cross, 2001; Tikhomirov, 1999, p. 349). This revelation refers to Leont'ev's object-oriented-ness which in its independent existence, may transform a subject's routine activity. However, in creative activity, Tikhomirov tells us an object may appear in two forms: 
As a new product of activity, a product that didn't exist before, and as an image of a yet to be created object. Whereas in non-creative activity the functions of comparision, reproduction, assimilation, and copying are of primary importance, in creativity activity the functions of construction, generation and creation of the new are most important. (pp. 349-350)

Tikhomirov's apparent black and white way of thinking about creativity was an important conceptual idea because it suggested that the dynamic creative design process was capable of creating new and shifting motives and goals, and through this, the course of the activity could change quite dramatically, thus suggesting originality. The link to non-creative activity was equally important because rather than it being percieved as a negative outcome, there is the suggetion that perhaps imitation and copying have a valid role to play within creative design process learning.

\subsection{Conclusion}

In conclusion of this chapter, theoretical frameworks of CHAT research have evolved through three generations, with the most recent version reconceptualised as AT (Engeström, 2001). Through AT and its analytical activity system approach, an emerging story relating to a particular AS may be told. AS models involving collective learning actions constantly evolve in response to tensions and contradictions discovered in between the separate component parts. Therefore, it is possible for a myriad of relationships to be analysed and articulated in some way, and a composite AS explanatory model presented (Foot, 2014). Theoretically and methodologically, CHAT/AT and its AS analytical model were argued to have the potential to express an open, fluid, historically situated picture of VCD students' engagement with creative design process learning situated in a particular project conducted in a classroom in China.

To the best of my knowledge, this research is the first of its kind that uses CHAT and its AS approach to analyse how VCD students in an undergraduate classroom in China engage with creative design process learning. Furthermore, this research was conducted in a completely different cultural context that at times confronted my 
New Zealand ways of doing and purpose. My certain ways of teaching were culturally situated within western-centred approaches, and so whilst this research was not strictly situated in a cross-cultural context per se, there was a requirement for cross-cultural and inter-cultural awareness to be understood by me. Therefore, recognising diversity and differences between my status as an outsider, my traditions and those of the teachers and students at HNCU involved consultations, and searches for teaching materials that were authentic, relevant, and local (Saris, 2018). My fascination of other cultures and respect for my participants' multiple perspectives and meanings aimed to present an account that is enriched with thick descriptions of complex, creative design process relations, interactions and issues currently in play (Geertz, 1973; Merriam, 1998; Stake, 1995). 


\section{Chapter 4. Methods}

This chapter outlines the way the theoretical framework of cultural historic activity theory (CHAT) shaped the research approach and methods used. To briefly recap, CHAT or activity theory (AT) which are used interchangeably, have evolved through three generations (Engeström 2001). Of interest was third generation AT in which an activity system analysis views the subject as an entity which may become understood through collaboratively constructed viewpoints. Activity networks, boundary-crossing and expansive learning may be articulated through expanded objects (motives). This concept contradicts traditional learning situations where teaching skills may lead to changes in individual learning behaviour, and when short-term goals or outcomes may not be realised when learning is being learnt as it is created (Engeström, 2001).

The research strategies that were used were intent on answering questions related to creative design process learning situated within transnational visual communication design (VCD) courses at Hunan City University (HNCU) in China. As outlined in Chapter 2, there is a dearth of literature, and very little in the way of empirical studies that focus on activities and creative design process learning within undergraduate education in China that is written in English. In response, one main research question and two sub-questions provided focus for the research.

\subsection{Activity theory and case study}

A number of features of AT and case study contributed to the rationale for their inclusion in the design of the research. AT and activity system analysis provided an approach capable of capturing the open, fluid, and historically situated nature of VCD students' engagement with creative design process learning at HNCU. Furthermore, the idea that the essence of complex data sets could be extracted, and findings communicated through graphic models, coupled with assertions of the compatibilities between an activity system analysis and case study research also supplemented the research design (Yamagata-Lynch, 2010). 
The way in which case study and activity system analysis are compatible begins with an understanding that case study methodologists usually emphasise the boundedness or boundaries of the case (Merriam, 2009). Attention is given to defining boundaries so that when an activity system analysis is either compared or combined within a bounded system (the case), object-oriented activities, or goal directed activities or the activity setting itself become the focus. Specifically, for activity theorists the unit of analysis for activity system analysis is object oriented activities that may be identified in terms of Rogoff's (1995) three planes (personal, interpersonal or community). Yamagata-Lynch (2010) defined this further; detailed activities are suggested to take place within "personal, interpersonal, or community/institutional planes" which effectively means there is no separation when object-oriented activities are studied as a bounded system (Yamagata-Lynch, 2010, p. 79). So while the boundedness of the cultural and historic contexts is perhaps more fluid and of greater interest in a CHAT analysis, case study is helpful in framing the research and the phenomena of interest which, in this case, is how Chinese students engage with creative design processes within the context of transnational VCD courses. Yamagata-Lynch's table depicts how an activity system analysis may be conceptualised with the bounds of a case study system, and how the unit of analysis is integrated within this approach (see Table 4.1).

Table 4.1. Activity system analysis and a case study (Yamagata-Lynch, 2010, p. 79).

\begin{tabular}{llc} 
& Activity system analysis & Case study \\
$\begin{array}{l}\text { Bounded } \\
\text { system }\end{array}$ & Object-oriented activities, goal-directed actions, or activity settings & Case \\
\hline Unit of analysis & $\begin{array}{l}\text { Object-oriented activities that could be identified within personal, } \\
\text { interpersonal (dyadic), or community/institutional planes }\end{array}$ & Case \\
\hline
\end{tabular}

For this research in particular, case study has been useful owing to its use of historiography techniques which involve questions about origins of practice, present practice, context, assumptions and impacts of the phenomena on the participants (Merriam, 2009). Recognisable here are links to activity theory which has similar aspirations, especially in regard to Engeström's (2001) expansive learning theories and questions which ask what is being learnt and how learning is being constructed. 


\subsection{Research design}

To answer the questions, the research was designed in two parts. Part one was designed to study students' engagement within the VCD approaches through two case studies of two pedagogical projects, a branding project and a cultural illustration project devised by myself and a colleague. Both projects were undertaken at separate times by the same 100 students who were enrolled in the second year of their undergraduate degree programme at HNCU. Whilst simultaneously teaching my allocation of classes, I conducted the research itself with 16 student participants who were situated in eight dyads within the two projects. The dyads consisted of two students working together; this is discussed in the next section. Part two of the research focused on understanding the cultural and historic pedagogical context. This comprised eight semi-structured interviews with Chinese teachers at the university, and observations in two Chinese medium VCD animation classes. This part of the research also included Chinese medium observations at a training school for grade three (last year of high school) art and design students in Changsha, the capital city of Hunan Province. All of the research was conducted with the aid of an interpreter (discussed in detail in the next chapter), and the nine-week data gathering period spanned one year, from September 2017 to September 2018.

In total, 24 primary participants were directly involved in both parts of the research and a further 380 secondary participants contributed data which will be discussed later in the chapter (see Table 4.2). 
Table 4.2. Research participants.

\begin{tabular}{|c|c|c|}
\hline Phase one & Primary participants & Secondary participants \\
\hline Case study one: Branding project & $\mathrm{n}=8$ (4 dyads) & $n=100$ \\
\hline Case study two: Cultural project & $\mathrm{n}=8$ (4 dyads) & $n=100$ \\
\hline \multicolumn{3}{|l|}{ Phase two } \\
\hline Semi-structured interviews & $\mathrm{n}=8$ (Chinese teachers) & \\
\hline Observations at $\mathrm{HNCU}$ & & $n=80$ \\
\hline Observations in Changsha & & $n=100$ \\
\hline
\end{tabular}

\section{Procedures for case studies: Phase one of the research}

The decision to design the research around two pedagogical projects in part one was both a theoretical and practical one. Stake (2006) suggests there are advantages in choosing fewer than four case studies. In choosing limited cases well, the opportunity arises for phenomena to be understood more deeply in terms of targeted complexities and "diversity across contexts" (p. 23). The selection of the two cases with the same 100 second year undergraduate students enabled a conceptual focus on developmental change in two different projects conducted at different times of the year (Stake, 2006). Furthermore, there was the potential to analyse how the creative design process within the context of transnational VCD courses in each case aligned or misaligned with what was discovered in the literature and has been discussed in Chapter 2 about creativity and creative design process learning (Yamagata-Lynch, 2010).

The two projects were used to analyse how creative design process learning occurs when students are exposed to a new pedagogy. The first project, the branding project, involved a fictional tea shop business and the second project, the cultural illustration project (based on the Miao minority tribe), involved designs for the creation of a deck of playing cards. For the full curriculum design and evidence of the teaching and learning from the branding project over the three weeks, see Appendix 6.1 (page 286), and for the Miao illustration project, see Appendix 7.1 (page 296). Each pedagogical project was framed within a prescriptive design thinking process using an adaptation 
of Kim and Lee's (2016) checklist and the Stanford d.school DT model to provide a structure for educational activities to take place. For the teaching and learning, all 100 students from each of the case study courses were randomly structured into dyads for certain aspects of the project work. In both case studies, my colleagues and I used the card game 'snap' to put the students into pairs at the beginning of the projects, and the research dyads were formed in this same way (Saris, 2018).

Dyadic student collaborations were included in the curriculum design to enable a sharing of concepts between the students, and to satisfy underpinning design thinking pedagogical processes (Stanford d.school, 2018). Furthermore, the methodological benefit of student collaboration for the research enables the observation of verbalisation, gestures or use of artifacts, and potentially uncovers covert creative and thought processes as they happen (Nasir et al., 2005). Explicitly for the research, an examination of dyads in conjunction with the other influencing activity system components, such as the division of labour, held the potential to highlight individual and collective group actions and activities in the tradition of Leont'ev (1981). However, collaborative activies were rare at HNCU due to the institutional assessment requirement that each student be graded individually, and so we were obliged to ensure students were assessed individually.

\section{Case study participants}

For each of the projects the 100 students were divided into four classes of 25 students. Two New Zealand teachers, myself and a colleague, were each allocated two classes by Chinese administration staff, and each project was taught over a three-week period (48 hours of classroom contact per student). Whilst teaching, I conducted the research itself with eight student participants from each of my two classes from both the branding and cultural projects identified by class numbers 203 and 205. Table 4.3 details information about the case study participants including pseudonyms that were used in place of student names in the thesis. Consent processes and other ethical considerations including the recruitment of the case study participants are discussed further on in this chapter. 
Table 4.3. Case study participants.

$\begin{array}{lllllll}\text { Case study } & \text { Class no. } & \text { Dyad no. } & \text { Pseudonym } & \text { Gender } & \text { Ethnicity } & \text { Age } \\ \text { Branding } & 203 & 1 & \text { Amy } & \text { Female } & \text { Hui } & 19 \\ \text { project } & 203 & 1 & \text { John } & \text { Male } & \text { Han } & 19 \\ & 203 & 2 & \text { Lily } & \text { Female } & \text { Manchu } & 20 \\ & 203 & 2 & \text { Connor } & \text { Male } & \text { Han } & 19 \\ & 205 & 3 & \text { Hana } & \text { Female } & \text { Han } & 20 \\ & 205 & 3 & \text { Michael } & \text { Male } & \text { Han } & 20 \\ & 205 & 4 & \text { Ricky } & \text { Male } & \text { Han } & 20 \\ & 205 & 4 & \text { Simon } & \text { Male } & \text { Han } & 19 \\ \text { Cultural } & 203 & 1 & & & & \\ \text { illustration } & 203 & 1 & \text { Will } & \text { Male } & \text { Han } & 20 \\ \text { project } & 203 & 2 & \text { Matt } & \text { Male } & \text { Han } & 20 \\ & 203 & 2 & \text { Aaron } & \text { Male } & \text { Han } & 20 \\ & 205 & 3 & \text { Rebecca } & \text { Female } & \text { Han } & 20 \\ & 205 & 3 & \text { Nina } & \text { Female } & \text { Han } & 19 \\ & 205 & 4 & \text { Jasmine } & \text { Female } & \text { Han } & 20 \\ & 205 & 4 & \text { Carl } & \text { Male } & \text { Han } & 19 \\ & & \text { Susan } & \text { Female } & \text { Han } & 20 \\ & & & & & \\ \end{array}$

\section{The cultural and historic pedagogical context: Phase two of the research}

The examination of the cultural and historic pedagogical context was devised as my research journey progressed. The decision to include the teachers' voices came about after I visited a freshman (first year) animation class towards the end of the data collection phase of the second case study. Further background about this visit and my observations on that day are presented in the following chapter. After careful consideration and informal conversations with colleagues from New Zealand, a teacher from northern Europe, and other VCD Chinese teaching staff, and after consulting with my supervisors, it was agreed to situate the findings from the two case studies in the context of the perspectives of the Chinese teachers at HNCU. This decision required an amendment to the ethics application, but at the time emergent findings from the case studies were so intriguing that this felt like an opportunity not to be missed rather than a burden. Eight semi-structured Chinese teacher interviews and observations in two Chinese medium animation classes at HNCU comprised this second phase of the research. The teachers at HNCU were diverse in the design 
disciplines that they taught at the university, and in their years of teaching experience (see Table 4.4).

Table 4.4. Chinese teacher participants.

$\begin{array}{lllc}\text { Pseudonym } & \text { Gender } & \text { Design discipline } & \begin{array}{l}\text { Years } \\ \text { teaching }\end{array} \\ & & & 19 \\ \text { Zhu } & \text { Male } & \text { Advertising design and history of fine arts } & 18 \\ \text { Ang } & \text { Male } & \text { Corporate identity design } & 18 \\ \text { Ming } & \text { Female } & \text { 2-dimensional and 3-dimensional design including packaging design } & 18 \\ \text { Yin } & \text { Male } & \text { Environmental design } & 17 \\ \text { Ting } & \text { Female } & \text { Animation } & 14 \\ \text { Lei } & \text { Female } & \text { Packaging design and calligraphy } & 5 \\ \text { Shi } & \text { Female } & \text { Graphic design / visual communication design } & 5 \\ & \text { Female } & \text { Animation and illustration } & 4\end{array}$

The 80 students that I observed in the two Chinese medium animation classes at HNCU were first year VCD students; their ethnicity, gender and ages were similar to those of the case study participants.

At the time of the teachers' interviews a further opportunity arose to visit a training school for grade three (last year of high school) art and design students in Changsha. Therefore, the research design was further expanded to include observations that were made there. Similarly, the students at the training school in Changsha were similar in ethnicity and gender to the students I observed at HNCU, but they were one or two years younger than the HNCU students.

\subsection{Data collection and purpose}

Data for the analysis were collected from four main sources: video stimulated recall interviews (VSRI n=16) with eight dyads; written reflections from the two case study classes $(n=200)$; Chinese teachers' semi-structured interviews $(n=8)$; and observations recoded manually in a notebook in Chinese medium (Mandarin) classrooms at HNCU $(\mathrm{n}=80)$ and at the training school for grade three art and design students in Changsha $(n=100)$. 


\section{Video stimulated recall interviews (VSRI)}

The VSRIs involved discussing instances captured on video, photographic documentation, and observations and reflections recorded as field notes from six weeks (192 classroom hours) of teaching at HNCU across the two case studies, the branding and Miao cultural projects. A total of eight recall interviews were conducted. The purpose of the video observations, photographic documentation, and observations and reflections was to provide context and support data for emerging findings, and to help to visually capture the students' creative design process for reporting findings. The purpose of the recall interviews was to provide data about:

1. How students physically enact creative design process,

2. How students interact with classmates, interpreters and NZ teachers in the classroom,

3. Creative design process learning and interactions from the perspectives of the dyads.

VSRI was selected as the main method for the two case studies due to its potential for capturing and examining instances of creative learning processes. VSRIs are particularly apt for complex contexts characterised by novelty and ambiguity (Lyle, 2003; Naidoo \& Kirch, 2016). The method uses retrospective reporting and investigates cognitive processes by prompting recall of current thinking to elicit motivation and thought processes. VSRI has been widely used in educational research, with both visual images and spoken words providing data for discussion (Mackey \& Grass, 2015). Mindful of the caution that VSRIs should be conducted within two to three days to ensure accuracy, and long-term recall bias did not result in a reliance on tacit knowledge or secondary ordering of cognitions, the VSRIs were conducted where practical within this timeframe (see Table 4.5) (Bloom, 1953; Mackey \& Grass, 2015). 
Table 4.5. VSRI timeframes.

\begin{tabular}{|c|c|c|c|}
\hline Case study & Week one & Week two & Week three \\
\hline $\begin{array}{l}\text { Branding project } \\
\text { September (09) } 2017 \\
\text { Class 203: Dyad } 1 \text { and } 2 \\
\text { Class 205: Dyad } 3 \text { and } 4\end{array}$ & $\begin{array}{l}\text { Project began } \\
06 / 09\end{array}$ & $\begin{array}{l}\text { Recruitment: } \\
\text { Thursday 07/09 } \\
\text { Video recordings: } \\
\text { Friday 08/09 } \\
\text { Thursday 14/09 } \\
\text { Friday 15/09 }\end{array}$ & $\begin{array}{l}\text { VSRI interviews: } \\
\text { Monday 11/09 } \\
\text { Friday 22/09 } \\
\text { Project ended 26/09 }\end{array}$ \\
\hline $\begin{array}{l}\text { Miao cultural project } \\
\text { May (05) - June (06) } \\
2018 \\
\text { Class 203: Dyad } 1 \text { and } 2 \\
\text { Class 205: Dyad } 3 \text { and } 4\end{array}$ & $\begin{array}{l}\text { Project began } \\
21 / 05\end{array}$ & $\begin{array}{l}\text { Recruitment: } \\
\text { Tuesday 29/05 } \\
\text { Video recordings: } \\
\text { Wednesday 30/05 } \\
\text { Thursday 31/05 }\end{array}$ & $\begin{array}{l}\text { VSRI interviews: } \\
\text { Monday 4/06 } \\
\text { Project ended 07/06 }\end{array}$ \\
\hline
\end{tabular}

For the branding project a total of approximately eight hours of video observations were filmed in the classroom on my iPhone 6 . The phone was mounted on a small tripod and positioned on a stack of wooden stools normally used for seating. This same procedure was repeated for the Miao cultural project, hence a total of approximately 16 hours of data were captured (see Table 4.6 for specific time durations).

Table 4.6. Video capture and interview time durations.

\begin{tabular}{|c|c|c|}
\hline Case study & No. video captures / total duration & VSR Interview durations \\
\hline \multicolumn{3}{|l|}{ Branding project } \\
\hline Class 203: Dyad 1 and 2 & $\begin{array}{l}6 / 28: 44,45: 34,43: 38,34: 30,55: 14,36: 32 \\
=256: 78\end{array}$ & Dyad 1: $47: 50$ \\
\hline Class 205: Dyad 3 and 4 & $\begin{array}{l}6 / 28: 13,30: 13,29: 42,46: 21,37: 07,50: 23 \\
=221: 19\end{array}$ & $\begin{array}{l}\text { Dyad 3: 63:43 } \\
\text { Dyad 4: } 34: 32\end{array}$ \\
\hline \multicolumn{3}{|l|}{ Miao cultural project } \\
\hline Class 203: Dyad 1 and 2 & $\begin{array}{l}6 / 38: 09,46: 36,35: 58,51: 33,43: 38,54: 40 \\
=269: 14\end{array}$ & $\begin{array}{l}\text { Dyad 1: 58:42 } \\
\text { Dyad 2: 01:16:36 }\end{array}$ \\
\hline Class 205: Dyad 3 and 4 & $\begin{array}{l}4 / 01: 29: 57,33: 06,39: 02,48: 58 \\
=250: 23\end{array}$ & $\begin{array}{l}\text { Dyad 3: 59:28 } \\
\text { Dyad 4: 48:23 }\end{array}$ \\
\hline
\end{tabular}

There were differences in my management of the video recordings and timings between the capture and the interviews. For the branding project, the actual research 
was conducted across the entire three weeks, and the eight hours of video capture were spread across two days, one week apart, in order to capture two of the design thinking phases, ideation and prototype (see Appendix table 1, page 286). The first interview with the dyads took place within two days, but the second interview took place one week after the final video recording was made. I discovered that the practicalities of teaching and managing the video capture simultaneously was difficult at times, and this led to a re-ordering of the procedure for the Miao cultural project. For this project the ideation and prototype phases were conducted on consecutive days, a Wednesday and a Thursday (see Appendix table 2, page 296), and the interviews took place the Monday following. There were no classes for the students timetabled on Fridays and so the three to four days that elapsed in between were beyond my control. Despite this, a more succinct arrangement for the video capture meant the teaching and research activities were better managed and I was able to spend more time reviewing the footage. I recorded notes and observations manually in a notebook as I watched the students' activities, and some of the data segments were used in the recall interviews. An iPhone 6 was used for the recall interviews, and mp4 files for the transcriptions.

My experience with VSRI was not without its challenges. First, I found it difficult to be a teacher and a researcher at the same time, especially in the branding project. Primarily the problem was to do with the video capture. My teaching routines entailed working progressively around the classroom from student to student in an attempt to ensure each student received learning support where it was needed. However, when working with the research participants, I realised that videoing myself would result in observations about my own teaching practice rather than capturing dyadic activities, and so I would turn the camera off (although there were inconsistencies with this practice). As a result, the video captures were not sequential in the strictest sense, which potentially limited observations of activities conducted through a continuum of time.

The second issue concerned the position of the camera. Prior to conducting the research at HNCU this procedure was tested in a New Zealand higher education institution classroom where I made the mistake of mounting a camera in front of two students, not four. Initially this was not an issue, because when a line of four learners 
(two dyads) sat side by side at a table and participated in physical idea generation exercises, it was easy to watch their process and interactions on the footage before the recall interviews took place. However, when the four students started working on their computers, it quickly became apparent that mounting the camera in front of them, meant what was happening on the computer screen was obscured (see Figure 4.1).

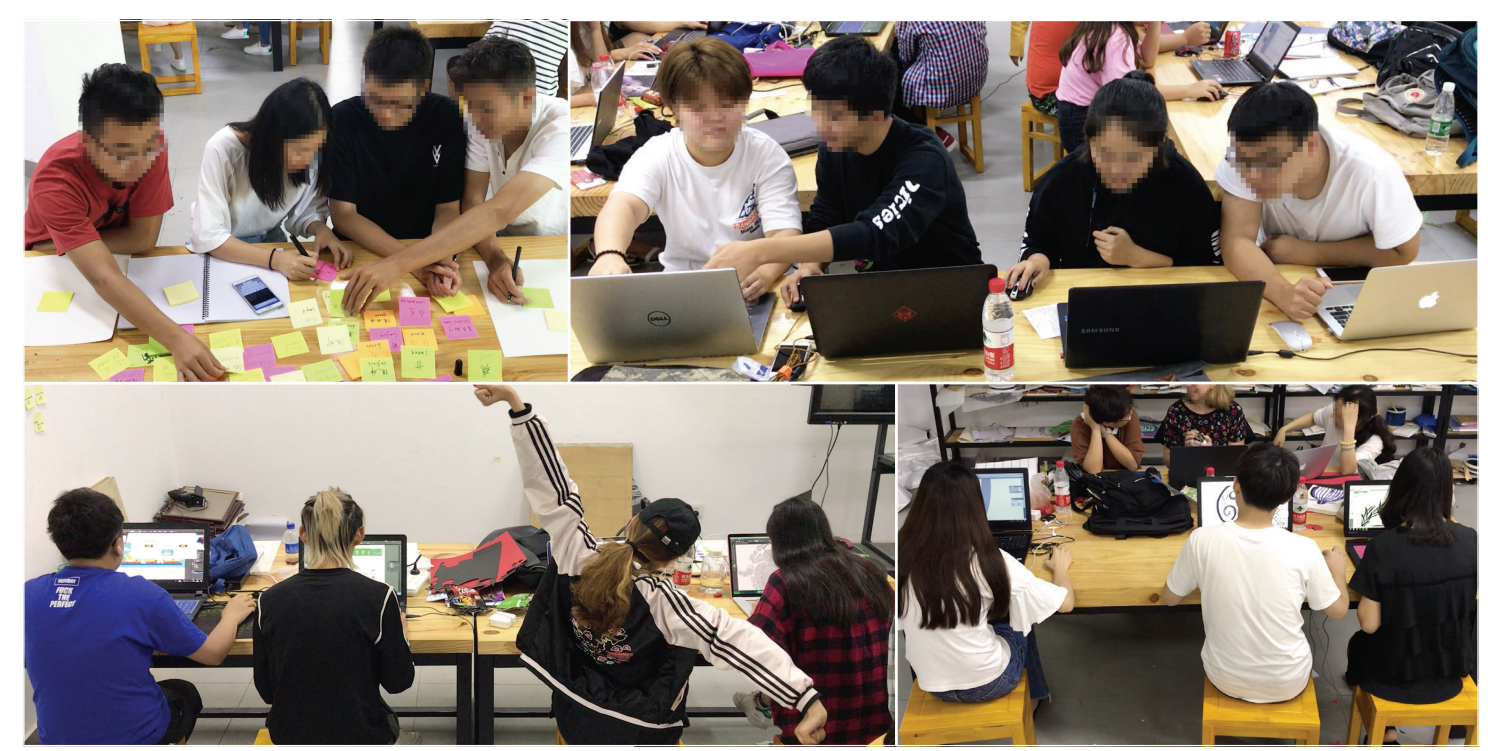

Figure 4.1. Video captures at HNCU (author images).

To mitigate this, the camera was repositioned behind the dyads, but this was not very successful either. Interactions were still able to be observed, but often the computer screen was obscured by the participants as they moved around on their seats. Added to this problem was that in one instance a participant moved to the other side of the table, and so observations of her actions were further compromised. In retrospect, and for future research of this nature, it may be prudent to use an on-screen video capture tool. This in combination with external video cameras to capture each of the individual students' process on the computer and student interactions may enable more detailed observations. Despite these issues, it was possible to adequately capture students' computer process activities and to call on this footage in the recall interviews. Furthermore, the benefits of capturing computer process work and student reactions and interactions simultaneously meant instances of 'happening at the same time' were examined, which in turn enabled more explicit explanations to emerge in the interviews. 


\section{The recall interviews}

Case study one focused on a branding project that aimed to create a visual identity system for a fictional tea business situated on the HNCU campus. Case study two focused on a playing card project that drew on international symbolism (card suits) and Miao minority tribe contexts. The background and curriculum design for both of these projects will be discussed further in the next chapter. Prior to the interviews taking place, I requested that the dyads bring all of their physical and digital work/laptop computers to the interviews in order to refer to this material. In many ways, using this material was more beneficial than using the video footage because participants were able to reference and discuss the examples in response to questions. Each of the dyad interviews was scheduled for one hour in duration; however, time became compromised when participants became distracted by watching themselves and would want to see the footage again, which often resulted in jokes and laughter in Mandarin. A further constraint was the need to take into account conversations that took place between the interpreter and participants. Although this was slightly frustrating, I wanted to be mindful and respectful of these interactions. However, one example of the benefits from having footage on hand for stimulating recall came about during one interview where it was evidenced that a student had fallen asleep (see Figure 4.2). The discussion that ensued was hilarious to say the least and is discussed further in the branding analysis chapter to follow.

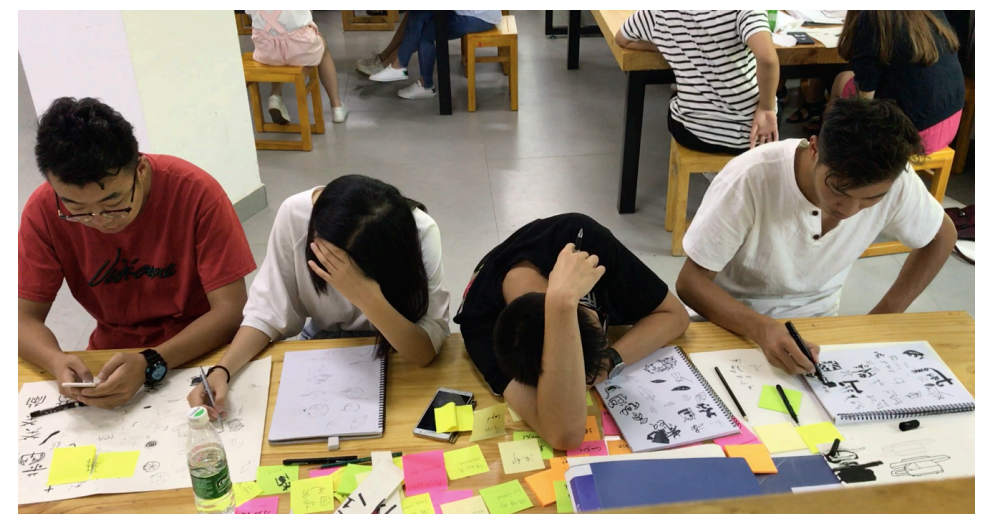

Figure 4.2. Video capture of a student asleep at HNCU (author image). 


\section{Written reflections from the two case studies}

Student reflections written in English and approximately 150 words in length from each pedagogical project (200 in total) were used in the analysis. The purpose of these data was to include perspectives from the wider class groups about each individual's creative design process learning and interactions. At the end of each project individual students from the four classes (both mine and my colleague's) submitted anonymous A4 pages of their reflections on how each had arrived at their final designs, what difficulties were encountered, and what helped their design processes. These 200 written reflections (see examples Appendix 4.4, page 284) provided valuable data and insights which either supported or contradicted the dyad assertions made in the recall interviews.

\section{Teachers' semi-structured interviews}

The purpose of the eight Chinese teachers' interviews and the observations at HNCU and in Changsha in a Chinese medium, was to underpin emerging findings from the two case studies with divergent perspectives and to gain a deeper understanding of the cultural and historic pedagogical context of the students.

I drew on Engeström's $(2001,2005)$ four questions from his emerging transformative and expansive learning theory to help with eight semi-structured Chinese teacher interviews which took place at HNCU in September 2018. In particular, my interest was focussed on what the VCD students were learning, and how they were learning. A sample of the questions used to tease out answers to my sub-questions included:

1. What is the most important thing you want to teach your students?

2. What is the most important thing you want your students to learn?

3. How do you think your students learn best?

4. What is the best way for your students to learn?

The advantages of using semi-structured interviews for my research worked on the notion that assumes participants would respond and define their world from a unique 
perspective (Merriam, 1998). This was important, because my position was firmly that of an outsider, and my world view was shaped by a New Zealand upbringing. The teachers' world was entirely different to mine, and so my questions were designed to elicit responses about how they aimed to develop creative design process learning in their classrooms. Moreover, this part of the research aimed to address both subquestions, and to provide insights to my main research question which asked, how do visual communication design (VCD) students at a university in China engage with creative design process learning?

\section{Observations in a Chinese medium (Mandarin)}

\section{Observations at $H N C U$}

At the time of the teachers' semi-structured interviews, I was invited by two of the younger female teachers to view student work that had been handed in for assessment. The observations and conversations that took place focused on aspects of their teaching practice, exploring teachers' perspectives and opinions about student outcomes, to help provide the background context for the research. The teacher interviews also gave me the opportunity to request access to their Chinese classes in order to observe teaching and learning practices. One of the younger female teachers readily agreed to this idea and so I observed two of her animation classes following the teachers' interviews. I sat quietly at the back of the classroom and recorded notes in a narrative that explained what I was seeing. When the teacher had finished her instructions to the class, I talked to her about her instructions to the students and requested that I take photographs of her teaching practice which she agreed to.

\section{Observations in a grade three art and design training school}

The opportunity to visit this grade three (last year of high school) art and design training school in Changsha was a privilege. My weekend visit was arranged by the Dean of HNCU who knew the owner, and over an approximately 4-hour period I photographed, observed, and conversed with the head teacher of this privately owned school. The teaching and learning programme in this particular training school lasted nine months, and long hours of instruction were evident. Further detail about this training school follows in the next chapter. The overall purpose of the visit to the 
training school was to provide information and context to address historicity (Engeström, 2001). The intention was to extend my understanding about prior learning and to collect data on factors or influences that may enable or constrain VCD learning experience at HNCU.

\section{Balancing combined teaching and research roles}

Being both teacher and researcher at the same time in the case studies had its difficulties owing to the inherent teaching tasks involving other students, which led to some difficulties in data collection, as was described on page 80 specifically relating to the video capture. This problem was not encountered when I conducted the eight teacher interviews, as the method for this phase of the research was 'non-technical' and involved semi-structured interviews and observations. The benefits of the observations conducted in these Chinese classrooms were that they enabled me to move from being a participant observer, which had been my role within the two case studies, to that of a non-participant /observer during this phase of the research (Creswell, 2013). I found the experience of being a non-participant liberating, owing to the affordances and opportunities to observe and formulate my ideas away from my teaching duties. However, despite this move out of the classroom in the second phase of the research, throughout my journals there is mention of my tiredness. For example, I have written, “when I did the interview today, I wish I hadn't been so tired due to the teaching demands and having to shift my thinking from what the students were doing, to interview mode within a short space of time. I think my follow-on questions in the interview could have been better thought through" (researcher reflection, Sept. 2018). Creswell (2013) suggests a combined role in research may lead to valuable inside views. To a certain extent I believe this to be correct; however, balancing combined teaching and researching roles is demanding. As a consequence, I came to understand how important it was to use multiple sources of data for the analysis.

To conclude this section a table is presented which summarises the data sources that were collected for the analysis, and their purpose (see Table 4.7). 
Table 4.7. Summary of data sources, participants and purpose.

\begin{tabular}{|c|c|c|c|}
\hline $\begin{array}{l}\text { Research } \\
\text { question }\end{array}$ & $\begin{array}{l}\text { Sub- } \\
\text { questions }\end{array}$ & Data sources and participants & Purpose \\
\hline \multirow{4}{*}{ 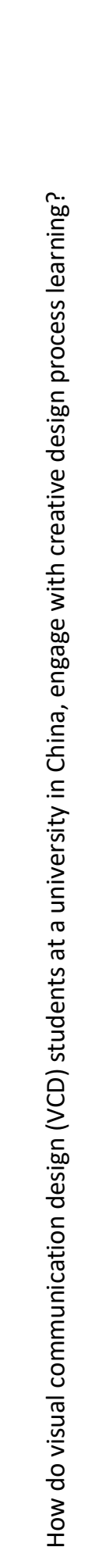 } & 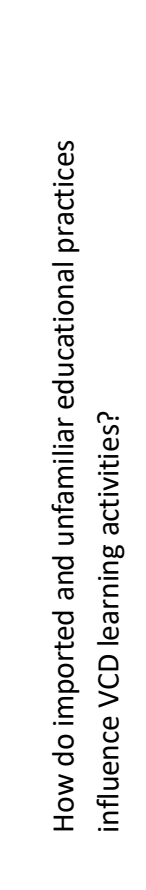 & $\begin{array}{l}\text { Video stimulated recall (VSRI) } \\
\text { Participants: } 16 \text { VCD students } \\
\text { Two CASE STUDIES (two CS): } \\
\text { BRANDING PROJECT, } \\
\text { MIAO CULTURAL PROJECT. } \\
\text { 1. Observations and photographic } \\
\text { documentation of VCD learning } \\
\text { process through six weeks (192 } \\
\text { classroom hours) of teaching at } \\
\text { HNCU within two CS. } \\
\text { 2. Approx. } 16 \text { hours of video } \\
\text { observations during two CS projects } \\
\text { of } 8 \text { dyads. }\end{array}$ & $\begin{array}{l}\text { Provide data about: } \\
\text { - how dyads physically enact creative } \\
\text { design process; } \\
\text { - how dyads interact with classmates, } \\
\text { interpreters and NZ teachers in the } \\
\text { classroom; } \\
\text { creative design process learning } \\
\text { and interactions from the dyads' } \\
\text { perspectives; } \\
\text { creative design process learning } \\
\text { and interactions from the wider } \\
\text { class groups' perspectives. }\end{array}$ \\
\hline & \multirow{3}{*}{ 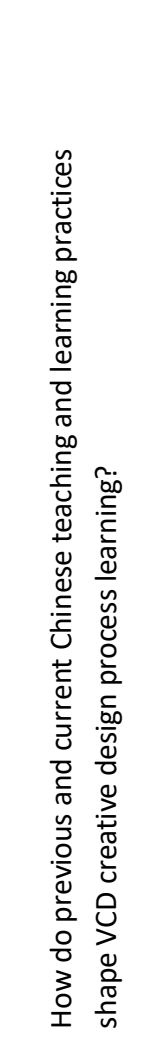 } & $\begin{array}{l}\text { 5. Observations, journal entries and } \\
\text { conversations with Chinese teachers } \\
\text { about student work }\end{array}$ & $\begin{array}{l}\text { Provide data from the teachers' } \\
\text { perspectives about: } \\
\text { - who they are teaching, } \\
\text { - why they are teaching students } \\
\text { the way they are, } \\
\text { - what VCD students are learning, } \\
\text { how they are learning } \\
\text { (Engeström, 2001). } \\
\text { Provide contextual information about } \\
\text { how aspects of Chinese teaching practice } \\
\text { outcomes are viewed. }\end{array}$ \\
\hline & & $\begin{array}{l}\text { Observations in Chinese medium } \\
\text { classrooms. } \\
\text { 6. } \begin{array}{l}\text { Observations in two Chinese } \\
\text { animation classes. }\end{array} \\
\text { 7. } \begin{array}{l}\text { Observations, photographic } \\
\text { documentation and conversations } \\
\text { with the owner of a 9-month high } \\
\text { school level 'training school.' }\end{array}\end{array}$ & $\begin{array}{l}\text { Provide contextual information about } \\
\text { Chinese teaching of creative design } \\
\text { processes to support data analysis. } \\
\text { Provide information, and context to } \\
\text { address historicity (Engeström, 2001). } \\
\text { Provide data on factors that may enable } \\
\text { or constrain VCD learning experience. }\end{array}$ \\
\hline & & $\begin{array}{l}\text { 8. Informal conversations } \\
\text { with colleagues at HNCU } \\
\text { and in New Zealand. }\end{array}$ & $\begin{array}{l}\text { Provide additional data, and context } \\
\text { from perspectives other than my own } \\
\text { in response to emerging findings. }\end{array}$ \\
\hline
\end{tabular}


The construction of Table 4.7 positions the research questions in relation to the particular data collection phases. Included at the base of the table are the informal conversations that took place through the data gathering and analysis phases with colleagues at HNCU and in New Zealand. The benefits of these conversations were that additional data, and context from perspectives other than my own about daily classroom teaching and learning at HNCU were reflected on and any harboured bias was potentially eliminated as findings emerged.

\section{Overall research design conclusion}

Data for the analysis were collected from four main sources within the two-part research design structure. In total, 24 primary participants were directly involved in both parts of the research. Video stimulated recall interviews were undertaken with eight dyads (two students together) comprising 16 of the primary participants. The analysis focused on VCD object-oriented activities of these students who participated in two pedagogical projects, a branding tea project and the Miao cultural playing cards project. The concept of incorporating dyads in the research design was made to satisfy design thinking pedagogical processes (Stanford d.school, 2018), and to enable collective object-orientated activities to be examined within an activity system (Foot, 2014). Owing to the emergent approach of activity theory, and, consistent with Engeström's (2001) activity theory multi-voiced and historicity principles, a further eight primary participants, the Chinese teachers, contributed data through semi-structured interviews. The teachers' voices emerged as significant as I moved into this unknown territory with the research. The data collection strategies for this part of the research were implemented to gain an understanding of what teaching methods were in play inside Chinese classrooms, what artefacts or tools were being used, and how the division of labour was enacted within this cultural and historic pedagogical context of the students. A further 380 secondary participants contributed data through written reflections from the two case study classes and observations that took place in Chinese medium (Mandarin) classrooms at HNCU and Changsha. Table 4.8 presents the overall research design. 


\section{Case study 1. Branding pedagogical project} 6 - 27 September 2017 (3 weeks) at HNCU

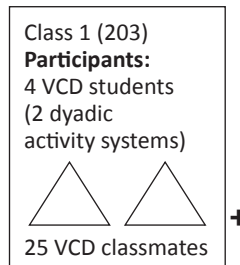

25 VCD classmates

Class 3

25 VCD classmates
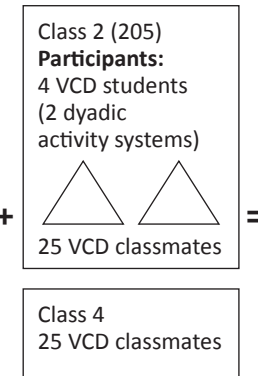

$=$

\section{Data sources}

1. Video

stimulated

recall

interviews

2. Observations

3. $100 \mathrm{VCD}$

student

reflections
Case study 2. Miao cultural pedagogical project 21 May - 8 June 2018 (3 weeks) at HNCU

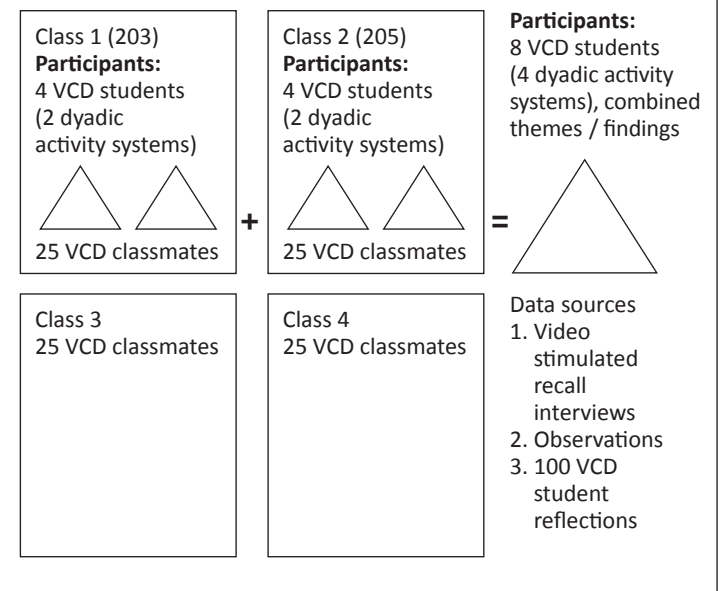

Phase two

\begin{tabular}{|c|c|c|c|}
\hline $\begin{array}{l}10-28 \text { September } 2018 \text { ( } 3 \text { weeks) } \\
\text { at HNCU and Changsha }\end{array}$ & $\begin{array}{l}\text { Participants: } \\
8 \text { Chinese VCD teachers } \\
\text { Data source } \\
\text { 1. Semi-structured } \\
\text { interviews at HNCU }\end{array}$ & $\begin{array}{l}80 \text { first year VCD } \\
\text { students } \\
\text { Data source } \\
\text { 2. Observations in two } \\
\text { Chinese medium } \\
\text { animation classes at HNCU }\end{array}$ & $\begin{array}{l}100 \text { Grade three art } \\
\text { and design students } \\
\text { Data source } \\
\text { 3. Observations in a } \\
\text { Chinese medium at a } \\
\text { training school in Changsha }\end{array}$ \\
\hline
\end{tabular}

Added to the teachers' voices in the analysis were mine and those of my teaching colleagues. My teaching colleagues included two New Zealand teachers who travelled with me to HNCU to teach the two projects, along with a Norther European teacher who was contracted to teach at the university at the same time. They were all aware of my research and in some cases, they helped, for example with recruitment. My dual roles, those of teacher and researcher, presented ontological and causal questions around Cartesian dualism (the independent mind-soul and body world view) and nondualism within CHAT scholarship (Robinson, 2017; Yamagata-Lynch, 2010). Nondualism within CHAT means the relationship between the organism (the human mind) and the environment (historic, social, cultural) maintains a "co-evolving relationship" rather than findings from these two things being discussed as separate or distinct entities (Yamagata-Lynch, 2010, p. 28). Criticisms against activity systems analysis relate to the ability to preserve a non-dualist perspective and method, and so for me it was vitally important to maintain an awareness of this in order to avoid "methodological dissonance" (Yamagata-Lynch, 2010, p. viii). 


\subsection{Ethics}

\section{Power asymmetries}

As both teacher and researcher, I was acutely aware of issues associated with power asymmetries not only in recruitment and interviews, but throughout the entire research process and through my interactions with each participant and the wider network of students, Chinese staff, interpreters and colleagues. A critically important challenge was to maintain sensitivity towards spheres of influence and Chinese characteristics such as miànzi (face) (Nguyen \& Tangen, 2016). Although I cannot claim insider status or a deep understanding of cultural concepts such as miànzi modifying views in Chinese culture, it was important I understood differences between individual, relational and group miànzi when conducting the students' and teachers' interviews (He \& Zhang, 2011).

\section{Mianzi}

Through my interactions with students in and from China, and collegial friendships with academics in China I have encountered the concept of miànzi. At times it can be troublesome to read what a Chinese person is feeling or thinking. Understanding miànzi from a western point of view can be difficult because it is a theoretical concept not easily transported from one cultural setting to another (Qi, 2017). According to Qi, the first Chinese references to face came from sociologist Hsien Chin Hu's 1944 writing, and the first reference came from an American missionary, Arthur Smith (1845-1932) who spent 54 years living in China. Smith is attributed to arousing interest in Chinese characteristics such as face and "losing face" (Qi, 2017, p. 6). In 1955, Goffman drew on the Chinese conception of miànzi in an attempt to define face. He described it as a self-imaged "pattern of verbal and non-verbal acts" with the purpose of maintaining "positive social value during particular contact" (p. 213). Goffman's discussion of face drew on miànzi in a general way in the sense that all cultures contain certain projections of self-image; however, the prominence of face in Chinese culture highlights the visibility and significance of the concept (Qi, 2017). Individuals in China are deeply embedded in social relationships and practices, and obligations are defined by roles, for example, in teacher-student relations. 
In these situations it is crucial that one's "face-state" depends not only on personal behaviour but the behaviour of others during social interactions (Qi, 2017, p. 9). What this means is that miànzi is intrinsically tied to renqing (interpersonal or relational behaviour), which is embedded in complicated spheres of social acceptance, status and prestige (He \& Zhang, 2011; Qi, 2017). This Chinese "collective face" is different to western conceptions of face where individual bad behaviour is not seen as "culturally limiting" in the way that it is in China (Qi, 2017). In group settings miànzi echoes political policy where there is a de-emphasis on individual wants and desires and an emphasis on harmony through "public face" (He \& Zhang, 2011, p. 2369). He and Zhang highlight that my actions as teacher and researcher had the potential to influence miànzi gain or loss and so to be "miànzi-sensitive" (p. 2368), I had to understand that my role at HNCU was as important as that of the interpreters, students and teachers.

\section{Working with an interpreter during the interviews}

Another ethical issue related to power asymmetries and imbalances which had the potential to arise related to my work with the interpreters during the interviews (Kvale, 2006). I aimed to mitigate these issues by being transparent with the interpreters about the purpose of the research, and I sought advice and support from interpreters throughout the research process when interacting with the participants. I found their advice invaluable; however, I did encountered a few issues. During the interviews in the first case study, often a long explanation from a student in Mandarin would result in a one or two sentence response in English from the interpreter; there is an example of this in the next chapter. Through this experience, I was aware that the interpreters may have been mediating participant responses in a way I could not understand. Therefore, I looked to the literature to ascertain how and what role an interpreter should play. It is suggested that the richness of participants' experiences and meanings may be misunderstood by researchers, and these are amongst the many issues associated with working with interpreters during interviews (Kosny, MacEachen, Lifshen, \& Smith, 2014). Moreover, cross-language and cross-cultural research focusses on the role of an interpreter or translator and how they might influence a participant's idea or belief statement (Caretta, 2015). But rather than thinking about these issues as problems, I preferred to think about an interpreter being "key 
informant" (Edwards, 1998, p. 202), I adopted an open approach in my work with the interpreters, recognising they too were contributing an important and added dimension to the nature of the data being generated. In the next chapter, Chapter 5, I introduce Hua and Shing (pseudonyms used) who both played a critical role in my research.

\section{Consent processes and other ethical considerations}

An English language information sheet and consent form to video and interview student and teacher participants were prepared for both phases of the research design. These were translated into Mandarin by a New Zealand colleague who worked in the international department at the higher education institution (see Appendix 4.1, page 277). For the interpreters an English language translating confidentiality agreement was prepared, but these were not translated (see Appendix 4.2, page 282). It should be noted that I myself completed the interview transcribing in New Zealand. For the Dean of Art and Music at HNCU I prepared a permission letter which was translated, and copies of ethics applications from both Victoria University (approved 25 July 2017, reference 24689) and the New Zealand higher education institution were given to him. For the second phase of the research, an extension from Victoria University was granted until 1 June 2019. At the end of these appendices is a table which summarises the dates consents were obtained from all participants. To further ensure all consent processes were covered, at the time of writing this thesis, I confirmed with the Dean of HNCU that I had his approval to include the name of the university.

The process of gaining informed consent from both the students and the teachers was an insightful experience, primarily because very few difficulties were encountered. This was surprising owing to my status as an expert potentially limiting participation, particularly student participation as I expected participants might have been shy in coming forward with offering information of merit or interest (Nguyen \& Tangen, 2016).

When teaching at HNCU our common practice was to combine the first two classes (mine and my colleague's) at the beginning of each morning or afternoon to explain the class learning activities. In this way, within the first week of both the branding and 
Miao cultural projects, together with the help of the interpreter I introduced my research to each group of combined 50 students. I explained my research and emphasised that participation would not compromise course grades as my colleague would assess all coursework. I discussed the benefits of case studies presenting composite pictures of practice rather than individual practice, thus confidentiality would be protected (Creswell, 2013). Furthermore, I explained that participants could withdraw their consent, or withdraw from the research up to six months after the VSRI interviews. In each case, when I gently asked if there were any questions no-one raised their hands, so I became quite worried about recruiting an adequate number of participants. As was planned I had enlisted help from my NZ teaching colleagues and left the classroom so they could recruit participants without my being present. The first time I did this for the branding project, I was surprised that after a very short time my colleague left the classroom with eight students. I re-entered the classroom and separated the two classes back into their respective classrooms and moved between the two rooms until my colleague returned. My colleague told me later that they all went to an empty classroom and read the information sheets. The students then took it in turns to draw a slip of paper out of a hat. These slips had a yes or no written on them. If the student drew a yes out of the hat, they readily signed the consent forms (see Appendix 4.1), and the ones that drew a no out of the hat, returned to class. When all of the volunteers had returned to class, I thanked them all in turn, and some who had drawn a no out of the hat expressed some disappointment that they would not be included in the research. This same process took place in the afternoon, so at the end of the day my first eight participants were confirmed. For the Miao cultural project, the same procedure was applied.

When it came to recruitment for the teachers' interviews, I approached the design school administrator for help because previously I had met only a handful of the Chinese VCD staff, and so did not know them well. She agreed without hesitation and emailed the information sheet to approximately 12 staff. Within 24 hours the administrator supplied a list of interviewees and times that the teachers and I would be able to meet. After this it was almost as if the floodgates opened, because those staff members came and found me, and others who had not been approached asked if they could be included. Suffice to say, I was humbled and overwhelmed at the same time. 
Ethics protocol procedures were applied (see Appendix 4.1, page 277) before each of the eight teacher interviews and without exception none of the teachers ticked requests to see their transcripts or the final thesis. They were far more interested in starting the interviews quickly to discuss and compare various aspects of teaching and learning. From my new connections with these staff I was able to ask if I could view their student work and observe their classroom teaching. This provided a valuable and enormously rich layer of meaning not only to my personal experience inside Chinese taught classes but to the research overall.

In managing broader ethical issues to avoid bias, harm and risk, I remained vigilant in all my interactions with other teachers, interpreters and students. I used triangulation for validation to avoid bias by continuously consulting a multitude of perspectives, which included: New Zealand colleagues, other western teachers at HNCU, interpreters, and VCD students situated at HNCU and in my New Zealand classrooms (Stake, 2006). However, as I sensitively discussed emerging findings, my participants' privacy and confidentiality had to be balanced with my concerns for authenticity, honesty and truth to be revealed in the thesis. Confidentiality has been protected in a number of ways: any images used in the thesis have been retouched to avoid identification of individuals, and pseudonyms have been used in place of actual names for all participants, interpreters, the business owner in Changsha, and any teaching colleagues that have been mentioned. All data are safely and securely stored and have not been revealed to anyone other than my supervisors, and all participants were assured of timely withdrawal procedures prior to any interviews taking place.

\subsection{Managing the space between trustworthiness and researcher reflexivity}

Merriam (2009) suggests qualitative educational studies are multifaceted and highly contextual; therefore, presenting accurate findings may be an issue due to the researcher being the prime instrument of inquiry. Similarly, Creswell (2013) suggests the background or positionality of the researcher "informs interpretation of the information in a study, and what [there is] to gain from the study" (p. 47). It is apparent from these suggestions, that any sort of bias must be carefully managed in qualitative studies in order to establish credible and trustworthy research. From this, an 
important question is raised. How have I held myself accountable to the "standards of knowing and telling of the people," that I studied? (Creswell, 2013, p. 257). This opens up a space between establishing trustworthiness and managing reflexivity. How can I be sure that my findings would be "sufficiently authentic . . . that I may trust myself on their implications" (Lincoln \& Guba, 2001, p. 120). How could I be sure that once people engage with the findings, especially the participants, that my interpretations were read as both honest and believable?

Managing this space between trustworthiness and researcher reflexivity was contingent upon openness and transparency. In order to address trustworthiness, I utilised a number of strategies to establish credible research: first, by careful conceptualisation of the research design; second, by triangulation, pertaining to use of multiple methods as sources of data, all of which has been described in this chapter; and third, through my reflexive practice. I have presented this reflexive practice in my writing throughout the thesis by richly describing situated contexts, and providing different perspectives about the same thing when this arose. Alongside this, there were continuous critical discussions with colleagues and supervisors and reading of the literature. Through this, my values and biases where carefully examined; I questioned how should I respond, or how to get to the heart of things when I became troubled. There were realisations that multiple layers and dimensions were often hidden within meanings; things needed to be prized apart, and from glimmers of understanding I delved deeper for more authentic insights to emerge. An example of this was the discovery of how 'perfection' could be interpreted in different ways (described on page 36). Furthermore, the temporal nature of the study meant I was embedded within the pedagogical projects as both researcher and teacher, over a sustained period, some 294 hours of classroom time, in addition to living and socialising with other teachers and interpreters outside these hours. This led to an approximate nine week focus on the research during the data gathering phases, which in turn led to valuable insider views (Creswell, 2013).

However, within this transnational context, there were times that it was not easy to be transparent or clear, especially when language barriers were present. As I do not speak Mandarin, I was heavily reliant on the interpreters I worked with. The realisation, that they too, were instruments of inquiry (Merriam, 2009) or key informants (Edwards, 
1998) as previously discussed, meant that issues of cross-language nuance, hierarchies of language power, and situated language epistemologies during the interviews, the analysis and interpretation of the transcriptions should be accounted for (Temple, 2008; Temple \& Young, 2004). I approached this in a number of ways. There were long conversations between myself and the interpreters about the nuance or a meaning of a word or expression, and various mobile phone translation applications were consulted in English and Mandarin in order to locate an understanding. Furthermore, the quotes in the findings chapters (see chapters 6-8) have not been adjusted from the transcriptions, they are in the original so there are deliberate mis-spellings and phrases which were carefully read by me for the interpretations. Once I returned to New Zealand, when I was unsure about anything, the interpreters readily responded to my double-checking or questions through WeChat. Guba (1987) highlights that findings in naturalistic evaluation result in many meanings, and so the question that should be asked, is “what have we learned?" (p. 23). My research approach aimed to present small snapshot of the creative design process phenomena situated in a single university setting in China. By establishing trustworthiness and managing reflexivity, the findings, and interpretations of the data discussed in the later chapters of this thesis, are unashamedly presented as my own. 


\section{Chapter 5. The research context}

\subsection{Study site}

Hunan City University is located on the outskirts of the city of Yiyang, in Hunan Province, China. Yiyang is built on the banks of the Zi River which flows into Lake Dongting. The size of city is not as overwhelming as some Chinese cities. Few high rise buildings exist, and with the exception of a few large hills on which two temples are located, it is largely flat. There are long tree and garden lined boulevard style roads that lead out of the city in different directions. One of these long roads leads out to the university. All along this road, the landscape rapidly changes with tall concrete apartment complexes being built. Each time I travel to Hunan the new apartment complexes move closer to the university grounds. Apart from the large mounds of building debris mixed with red-coloured earth, the landscape surrounding the university is green with small vegetable gardens and the occasional abandoned ceramic tiled house nestles inside swathes of bamboo. A large planting programme was undertaken in 2016 inside and around the environs of the university. My New Zealand colleagues and I watched in awe as the roads and grounds were planted with large uprooted mature trees, and seemingly overnight whole gardens and lawns would appear. Most of these trees survived, perhaps in part owing to the high rainfall and sub-tropical climate. There is a small lake inside the HNCU campus grounds which is presumably fed from a $\mathrm{Zi}$ River tributary.

The university buildings seemed typical of other Chinese universities I had visited; large and imposing concrete structures from the outside, inside they are dark and humid in the summer, cold and damp in the winter. The layout of the university is oriented on a north-south, east-west axis with the library building in the centre. The School of Art and Design sits in a separate purpose built complex in the south-west corner of the campus. The VCD classrooms are located on the second and third levels of one of the buildings. The classrooms were recognisable to me owing to the familiar layout and size to traditional New Zealand school classrooms. The student dormitory 
complex is located in the north-west corner of the campus grounds, and there are approximately four large food hall cafeteria buildings and a number of well utilised basketball courts and outdoor ping-pong tables in the vicinity of the dormitories. Most of the students live in this area, although some live at the north gate. The north gate is an older, more established area across the road from the north gate of the university. Many of the university teachers reside in this vibrant community where small restaurants and street-food stalls line the streets. Directly opposite the north gate is the south gate. This is the main entrance to the university, and the gates are flanked by traditional Chinese guardian lions.

\subsection{Teaching and learning timetables}

In the mornings during the summer, the teaching and learning day begins at $8 \mathrm{am}$ and progresses through a number of periods, ending at 11.40am (see Table 5.1).

Table 5.1. Timetabled morning hours (summer).

\begin{tabular}{llll} 
Morning & Duration & Afternoon & Duration \\
$8.00-8.45 \mathrm{am}$ & 45 minutes & $2.30-3.15 \mathrm{pm}$ & 45 minutes \\
\hline $8.45-8.55 \mathrm{am}$ & 10 minutes & $3.15-3.25 \mathrm{pm}$ & 10 minutes \\
\hline $8.55-9.40 \mathrm{am}$ & 45 minutes & $3.25-4.10 \mathrm{pm}$ & 45 minutes \\
\hline $9.40-10.00 \mathrm{am}$ & 20 minutes & $4.10-4.30 \mathrm{pm}$ & 20 minutes \\
\hline $10.00-10.45 \mathrm{am}$ & 45 minutes & $4.30-5.15 \mathrm{pm}$ & 45 minutes \\
\hline $10.45-10.55 \mathrm{am}$ & 10 minutes & $5.15-5.25 \mathrm{pm}$ & 10 minutes \\
\hline $10.55-11.40 \mathrm{am}$ & 45 minutes & $5.25-6.10 \mathrm{pm}$ & 45 minutes \\
\hline
\end{tabular}

In my experience, this kind of timetabling can be a taxing experience. A bell sounds frequently in the corridors to signal the break times. By the time I started conducting 
the research for this $\mathrm{PhD}$, after three previous stints of teaching at HNCU, I had become accustomed to the regime. But when I was working closely with the students, I would not hear the bell ring, nor would I encourage students to take a break. The students were either too polite to say anything or they themselves were engrossed in whatever task they were undertaking. Sometimes flow cannot be interrupted and I found the incessant breaks not conducive to a practicum-based studio learning experience, that I was more familiar with (Csikszentmihalyi, 1990; Wilson \& Zamberlan, 2017). The hours make for very long days; especially difficult are the long lunch breaks which are very different to my hurried sandwich eating lunch breaks in New Zealand. Students troop off to the cafeteria for their hot noodle or rice dish and then go back to their dormitories on campus for a rest. Similarly, Chinese staff eat a hot meal, then sleep on couches in their offices. The university corridors fall completely silent until a bell rings and a female voice announces over a loudspeaker that afternoon classes will resume in 20 minutes time.

When I first began teaching at HNCU, it was surprising to learn that in effect, students were scheduled to learn within one design 'specialism' each week, for three to four weeks, and then they would move on to the next specialism. The three-week branding project I was involved with began in week two of the autumn semester in 2017. Following the branding project, students moved on to a one-month field trip to the province of Guizhou. This trip was implemented by the university to enable students to experience minority cultural festivals. They participated in workshops run by local people in the villages of Guizhou, for example indigo dying and batik, and gathered reference material through photography and drawing for the Miao cultural project which was scheduled in the second semester of the academic year. Following the field trip, students returned to the university, and undertook a similar timetabled structure for visual design, and then animation:

- Branding (weeks 2-4)

- Guizhou cultural field trip (weeks 6-10)

- Visual design (weeks 13-15)

- Animation (weeks 16-18). 
There were no other design classes running at the same time as a specialism within the timetabled structure. The scheduled hours for specialism design learning comprised four learning blocks per week. Each block consisted of three hours and forty minutes, a total of just under 14 hours in one week. This same amount of time was scheduled for international English language testing system (IELTS) learning. A further seven hours and 20 minutes were added to these classroom hours for spoken English and Chinese history classes. Therefore, students were expected to be in a classroom environment receiving instruction from a teacher for approximately 35 hours per week. One single three hour and forty-minute block scheduled for physical education provided them some relief from sitting down for such long hours (see Table 5.2). In the wintertime there is a shorter lunch break, meaning the afternoon classes recommence at $2 \mathrm{pm}$, and the day ends at $5.40 \mathrm{pm}$.

Table 5.2. Timetabled days and hours (summer).

\begin{tabular}{|c|c|c|c|c|c|}
\hline Time & Monday & Tuesday & Wednesday & Thursday & Friday \\
\hline $8.00-9.40 \mathrm{am}$ & IELTS & Design Class & IELTS & Design Class & IELTS \\
\hline 10.00-11.40am & IELTS & Design Class & IELTS & Design Class & IELTS \\
\hline $2.30 p m-4.10 p m$ & Design Class & $\begin{array}{l}\text { Physical } \\
\text { Education }\end{array}$ & Design Class & $\begin{array}{l}\text { Chinese } \\
\text { History }\end{array}$ & IELTS \\
\hline 4.30pm - 6.10pm & Design Class & $\begin{array}{l}\text { Physical } \\
\text { Education }\end{array}$ & Design Class & & IELTS \\
\hline 7.30pm-9.10pm & & & Spoken English & & \\
\hline
\end{tabular}

Whilst I found the ten and twenty-minute breaks disruptive, it was important for the students to have their breaks. I could only assume that this relentless schedule with little variation contributed to their tiredness. Tiredness was an issue that was readily observable, with students often falling asleep in class. 


\subsection{Overview and pedagogical underpinnings for two case studies}

Visual communication design formats provided the foundation for the pedagogical approach for both case studies (Davis, 2016). Communication format approaches work on the assumption that core elements such as point, line and plane, and design principles, for example balance, emphasis and rhythm are understood and applied in the design process. Learning is transferred between courses of study, for example, from branding design to web design. However, design curriculum that adopts visual communication formats alone has been critiqued for its lack of learning scaffolding (Davis, 2016). There are similar issues associated with VCD courses which focus on formal and visual expressions of stylistic design, for example, creating patterns from typographic elements for a book cover design. This approach results in students developing concerns for "substance over style" (Kim \& Lee, 2016, p. 206). For these reasons, aspects of DT were used to augment the pedagogical learning. DT was discussed in detail in the literature review (see pages 23 and 31). The curriculum was designed to focus on the ideate, prototype and evaluation phases of DT, in order for a more iterative learning process to be enabled. For the most part, both projects attempted to place students into dyads or pairs in order for them to become comfortable with collaborative work as prescribed by DT. However, owing to the Chinese institutional rules, assessment had to be managed on an individual level; therefore, the curriculum design allowed for both dyadic and individual outcomes.

Data gathering took place over a total period of nine weeks at HNCU. Two pedagogical projects (two case studies) and the teacher interviews were analysed for the overall research (see Table 5.3). 
Table 5.3. Data gathering phases and dates.

\begin{tabular}{ll} 
Data gathering phases & Dates (3-week durations) \\
\hline Branding project case study & $6-26$ September 2017 \\
\hline Miao cultural project case study & 21 May-7 June 2018 \\
\hline Teacher interviews & $10-28$ September 2018 \\
\hline
\end{tabular}

The background context for each of the pedagogical projects is discussed next, beginning with the Branding project. Whilst conducting the teacher interviews, I taught the Branding project for a second time through the three-week duration.

\subsection{The branding project: Background context}

The idea for a pedagogical branding course to be integrated into the overall curriculum for the Hunan VCD students stemmed from assertions about the role of design for China's innovation industries (Liu, 2016). A new design related policy unveiled by the Chinese central Government in May 2015 proposed the Made in China 2025 strategy (Liu et al., 2018). The significance of the strategy was that it highlighted the role of design in the transformation of China's economy. In other words, it called for design to play a deeper role in enabling businesses to transition from manufacturing towards one of innovation (Don, 2014). Liu (2016) discusses Chinese innovation as an integrated form of product and service creation for the new knowledge economy, containing many facets and scenarios including engineering and cultural considerations. As China is viewed as a rapidly developing country, the industry economy (no design), the experience economy (from design as styling to design as process) and the knowledge economy (design as strategy) typically coexist within contemporary economic scenarios (Liu, 2016). The issue with the idea of co-existence is that China has not had the benefit of transitioning slowly from one economic phase to another (Staats, 2011). Staats argues that despite a glorious ancient history of creative inventions, the compass, gunpowder, papermaking, and printing, China's creativity is constrained by incremental rather than radical innovation. Johnson and 
Weiss $(2008,2011)$ suggest if China remains in the imitation and incremental innovation stages, there will be difficulties for the country in moving towards becoming a major player in the knowledge economy.

It is widely acknowledged in the literature that design, innovation and branding is the key to developing the knowledge economy. Neumeier (2009) introduced a simple flow diagram "design $>$ innovation $>$ brand $>$ loyalty $>$ profits" (p. 15) which positions brand at the centre of a strategically designed business process. To explain, the diagram begins with design and arrow. The diagram is premised on the notion that agile and adaptable businesses use brand to build business equity and foster loyalty, and they use design and innovation to create differentiated products and services to delight their customers.

Aaker and Joachimsthaler (2000) assert powerful forces dominate the purpose of branding including competitive pricing, a proliferation of similar products, and powerful global brands dominating market places. Gobé (2009) discusses the desire of people to experience emotional fulfilment rather than material satisfaction when it comes to consumption of products and services. He argues culturally relevant and socially sensitive innovative brands seek personal dialogues with consumers on issues that are meaningful to them. Arguably, this builds on Jensen's (1999) assertions that globalisation would move people beyond the knowledge economy to a dream society where future consumption of products would appeal to hearts, not heads.

Siu and Contreras (2017) highlight the issues brand initiatives wrestle with within the economic transformation in China. They contend it is a well-known fact that practising Chinese designers struggle with business owners' lack of understanding about the value of design in providing intangible customer benefits in product and service development, which in turn may be leveraged for branding purposes. Consequently, there is an implication that strategically designed business processes such as Neumeier's (2009) version are failing in China. The challenge, not only for Chinese businesses but also for design pedagogy, is in aligning the Made in China 2025 vision with either organisational or institutional demands, for example, maintaining commercial viability. At the same time, Chinese design education grapples with 
negative criticisms that assert a focus on outcomes, and assessment takes precedence over critical thinking and creative design process learning to promote innovation (Dineen \& Niu, 2008; Zhu, 2015). Therefore, it seemed to me that although one could not assume any sort of difference could be made, conducting a branding project that had some meaning and relevance for the HNCU students may provide them with the tools to successfully engage with these challenges.

\section{Choosing a topic for the branding project.}

Choosing a branding topic for the project which was familiar to the students came about owing to the discovery that this area in Hunan Province is well known for its Anhua dark tea. The tea is grown in Anhua, a hilly region about one and a half hours drive from Yiyang. Chinese staff from HNCU had introduced us to the 'tea street' located in Yiyang, where we spent weekend hours enjoying the hospitality of local people in this area. A sculpture outside on the street depicted three men rolling the dried dark tea into a cylindrical shape wrapped in a woven bamboo package. Although I have never seen it, I was told that these cylinders are carried down from the mountains on horseback to trading streets such as the one in Yiyang. Here cylindrical sawn-off cross sections, or blocks of dried dark leafy tea were boiled or 'washed' once, and then boiled again before it was safe to drink. An intricate washing of cups, pouring hot water and old tea over ceramic 'tea pets' for example, mythical water-buffalo, or buddha figurines followed. Drinking copious cups of various coloured teas including: green, red and Anhua dark tea, we became fascinated by these local tea traditions and customs (see Figure 5.1).

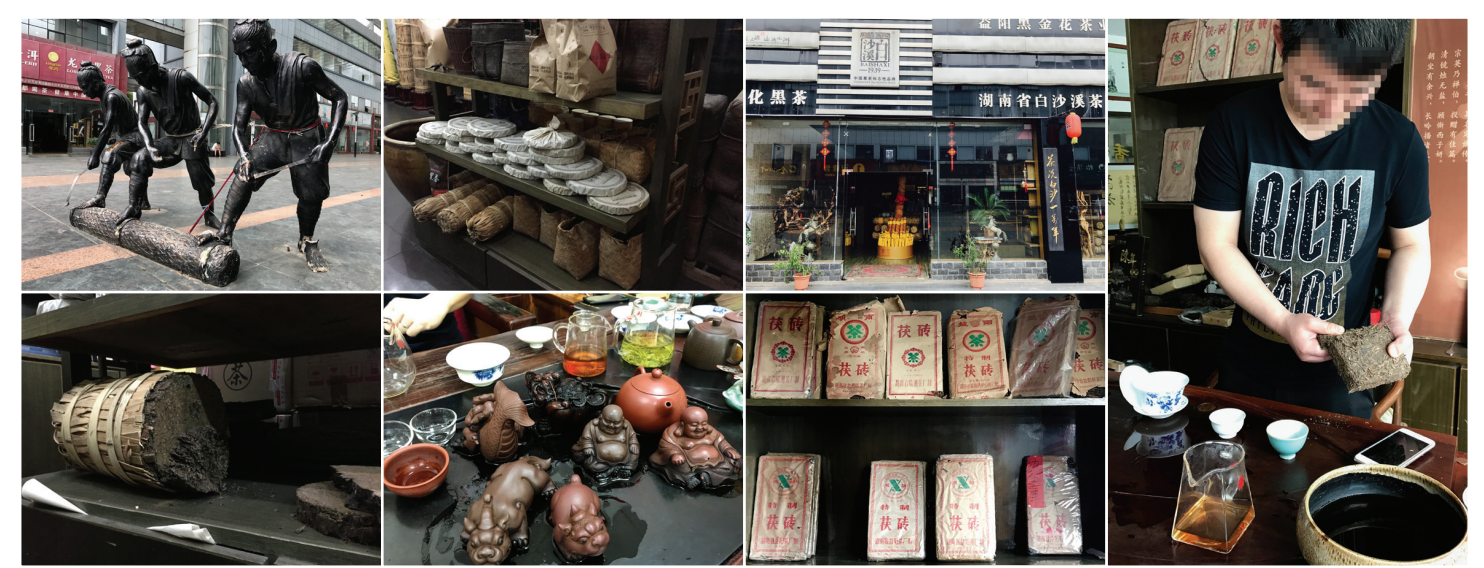

Figure 5.1. Tea street in Yiyang (author images). 
As a result of our tea street encounters my New Zealand colleague and I devised the pedagogical project, with emphasis placed on a branding tea experience. The course used a hypothetical Chinese tea shop business, such as the ones we visited in Yiyang, as a basis for learning. The project involved designing brand identity elements, for example, a name, logo, colour palette and texture/pattern swatches. The brief suggested the hypothetical tea shop would be located within the Art and Design building at HNCU. Therefore, consideration of alternate target audiences would be required when it came to the designs. The branding would have to appeal to staff such as us, who would potentially visit the tea shop, as well as other VCD students, international visitors and staff, and Chinese teachers.

\section{Branding teaching plan}

For the following discussion see Appendix 6.1., Branding project: A China tea house (page 286). This appendix provides a detailed explanation of the teaching curriculum structure and evidence of the students' learning from the project. Details about the four hours of video recording which took place during the DT ideate and prototype phases of the project for the $\mathrm{PhD}$ research are documented at the beginning of this appendix.

In September 2017, I was scheduled to begin teaching the branding project. For the project I was assigned 51 second year students, divided between two classes, comprising 25 students in one class, and 26 students in the other class. Twelve fourhour lessons were devised for the project so each individual student could expect 48 hours of teacher directed hours over the three weeks. To accommodate all 51 students situated within the two classes, I taught the curriculum twice through a schedule that stipulated the teaching be conducted four days a week ( 32 hours contact per week for three weeks, a total of 96 hours).

The very first day of teaching was chaotic owing to a bereavement in my colleague's family which meant he was unable to fly to China with me and begin teaching on the first day. Therefore, I combined my class with my colleague's assigned morning class and repeated this with the afternoon classes. Effectively there were in the vicinity of 50 students, two interpreters and me in the morning classroom, and the same again in the afternoon. Four tasks were completed on that first day: 
1. The project was introduced, and I delivered a short lecture which covered contextual and theoretical aspects of brands and branding;

2. Each class decided and voted on a name for their tea shop/café. I had prepared a number of international tea brands and project exemplars for inspiration. Possible names that were offered as thought starters included tea house, Tbar, and Tea lounge;

3. Within class groups the students played the card game of snap with the aim of assigning pairs (dyads) for some of the class work;

4. Once each student had 'won' their partner, they were asked to start problem structuring. This involved discussions about the empathise and define stages of DT. The students set about defining the problem and setting themselves design goals.

For homework that night, the students were asked to prepare for the next class by collecting items and tea related materials for the project, such as tea leaves/pets/pots/cups and drawing tools and media such as pens, pencils, paint brushes, sticks/twigs, paint and ink. Despite the chaos with such large numbers of students in one confined space on that first day, with support from the interpreters each class decided on a name for their tea shop. My 203 class decided to work on the brand name tea friends and the other class chose $T$ one (1).

The second class was conducted in the ideate phase of DT. The time was spent generating an extensive variety of drawings. There was much hilarity when I moved the students away from sustained drawings of the tea materials. I asked them to use their sticks and twigs with ink to generate rapid sketches, some with their eyes open, and some with their eyes closed. I timed the sketches beginning with 2 minutes, 1 minute 30 seconds, 30 seconds, 15 seconds. It became apparent that the students were accomplished in their drawing abilities. To my eyes the results were extraordinary, yet for the most part the students dismissed their work. I was left with the impression that they were not satisfied, because all they seemed to see were mistakes, and they complained their work was not perfect, and much of the work was left on the floor or the tables when the class ended. The aim of the class had been to generate original work that could be used and manipulated for brand identity elements such as a logo 
design, but at that point it appeared the students were not convinced of the merits of this idea.

In the third class a quick 30-minute exercise was introduced where students used postit notes and internet research to consider what the 'brand essence' of their tea shop/café should convey to a potential customer or target audience. For example, the hypothetical business could be cozy/warm, fresh/light, or perhaps a theme which incorporated Chinese medicinal plants could be used. Following this, the remaining two and a half hours were used for further ideation phases. Here, the students were encouraged to sketch rough thumbnail drawings. This in itself was challenging because my idea of thumbnails meant that there might only be a few sketched lines to indicate a form or shape, whereas the students tried to spend longer periods of time drawing things, so the objects were recognisable. The aim was to first sketch out conceptual ideas that linked the post-it note brand essence specifically to a pictorial tea object, for example a 'warm' coloured tea cup. Secondly the aim was to conceptualise typographic ideas by mixing English letterforms and Chinese character forms which linked to the brand essence. We referred back to the exemplars I had used at the beginning of the project. An example of this typographic approach showed the English word 'book' had been combined with the Chinese character for book to make a bilingual logo design. Despite much prompting, the students found this difficult, and it was not until I asked the interpreter to draw the Chinese characters for 'tea' and 'warm' on the blackboard and I myself started to morph English letters into the character to envisage new meaning on the blackboard that some of the students gained confidence to try the approach.

The fourth class was assigned as a technical learning class in order for the students to refresh their Adobe Illustrator software skills. My colleague and I had prepared worksheets to aid learning of the various tools and techniques provided by this software programme. At the end of this class the students were asked to make scans of their drawings from the second class in readiness for digital manipulation of the brand identity elements. To my surprise, most left the classroom before the bell went. The interpreter informed me that the students had to go to the printer to scan their work. On further investigation, the printer turned out to be a small privately-owned copy shop at 
the north gate. The owner had three photocopiers which could also be used for scanning and he did the job for the students. I did not think about this very much at the time, but later when I was back in New Zealand teaching, I discovered an international Chinese student standing perplexed by the photocopier. He told me it was the first time he had to make a photocopy for himself.

The remainder of the classes for the branding project attempted to follow an iterative ideate/prototype/test process. However, I found that once the students moved on to the computer designs, they were reluctant to revisit an earlier DT phase. Arguably, the time was short, and it was difficult to allocate equal amounts of individual attention to each student. However, I found that as I moved from student to student, by the time I got back around to each one, their work had not moved far from where it was when I had previously left them. I spent a lot of time trouble-shooting computer problems which was frustrating, and I struggled with the ubiquitous mobile phone use. Despite this, there were some stand-out results where the students became completely engrossed in what they were doing. This was especially apparent when a dyadic collaboration sparked, and I could sit down with a pair to brainstorm and sketch new ideas together.

\section{Reflections and realities of the branding project}

The timing of this particular project was not without its challenges. The course commenced in the second week of a new Chinese academic year. The students were second year students, referred to as sophomores. As the branding project class started after the summer vacation, it was extremely hot with temperatures reaching upwards of 30 degrees Celsius. Luckily both classes were conducted in air-conditioned rooms. All class groups are assigned a single classroom for the three- or four-year duration of their studies. As a result, teachers move between different rooms and class groups. In 2018, the year after I had completed the case study research, I went back to Yiyang to conduct teacher interviews for my research and to teach the branding project for a second time. Neither of the classrooms assigned to the new cohort groups was airconditioned. This was in stark contrast to the year before when I had been filming and interviewing student participants for the first case study. For me personally the heat in 
2018 was almost unbearable, but it did not seem to affect the students. I did not discern or observe any noticeable difference in student engagement between the two cohorts.

It had been surprising to learn, that at the beginning of a new Chinese academic year, compulsory military training took place on the HNCU campus. I was informed by Chinese staff this happens all over China. This only affected first year, 'freshmen' students. Throughout the grounds of the university, class groups of approximately 25 students were instructed in a disciplined manner about various marching techniques. They were all wearing the same camouflaged clothes. Once I came across all the uniforms neatly folded and laid out on a basketball court. On the edge of the basketball court there was a row of water bottles all neatly lined up. Often, when I was walking through the grounds during a mid-day break, I would stop and watch. In 2017 my natural reaction was to just observe quietly in the shade of a tree, but once when I walked past a group that were marching past me, a couple of the students grinned and said 'ni hao' (hello). This broke the ice and gave me the confidence to interact with the students more readily. The reactions of the students were charming, and the lead training officers would wave back or smile. It seemed when I returned to the university to teach the branding paper in 2018 , regulations had relaxed a little. Whereas the previous year, all were wearing military uniform, in 2018 a number of groups were wearing t'shirts and track pants or jeans. My rudimentary Mandarin language skills also recognised that the groups were counting in Chinese yi (one), èr (two), san (three) as they marched (see Figure 5.2).

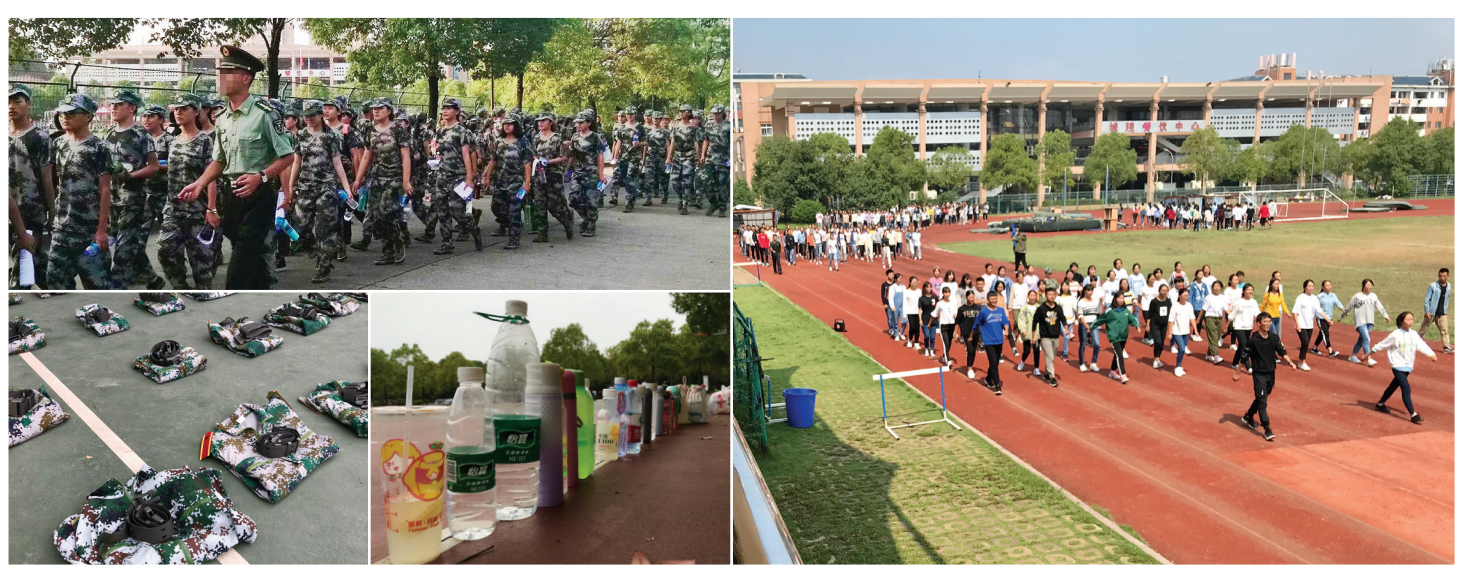

Figure 5.2. Freshmen military training at HNCU, September 2017 and 2018 (author images). 
The reason the freshmen's military training has been discussed was due to some of the sophomore students requesting leave from the classroom to participate as 'trainers'. In 2017, a high achieving student and research participant would race out of the branding class at various times to 'train' her class group. She would arrive back in class in her military uniform, sit down and continue her design work with or without her dyad, depending on the stage we were at within the design thinking process.

In 2018, I was approached again by one of the students requesting permission to leave class to be a trainer. I agreed, thinking the student would still be able to maintain a focus on their VCD learning, since that had been my experience in the previous year. However, things did not work out in quite the same way. The student did not return to class until the beginning of the third and final week of the teaching, despite my persistent requests regarding his whereabouts. He showed me some minimal work, and requested I sign a form that was written in Mandarin giving permission for him to attend military duties and that he would pass his course. This was somewhat of a shock as I felt morally compromised with the idea of passing work that was not at a suitable level, and I therefore, refused to sign the form. One day later, the student arrived in class with the lead VCD manager and an interpreter. It was explained that I had to sign the form because the student's other class teachers during the period of military duty had agreed to the terms. I suggested an extension and that work be handed in the day I would leave to travel back to New Zealand. This was eventually agreed, and the issue was resolved somewhat. Whether the work was of a high enough standard was questionable. As I had been conducting the Chinese teacher interviews whilst teaching the branding course for the second time, I had been in discussion about passing or failing students. Therefore, I was beginning to understand the complexities around supporting Chinese student success.

\subsection{The Miao cultural project: Background context}

The idea for the Miao cultural project was initiated in 2016 by Long Xiangping, who was the Head of the School of Art and Design at HNCU. One day, when I and my colleague were drinking tea in Mr Long's office, he showed us his self-published book celebrating Miao culture. In another glossy tourist book, we viewed Miao village and 
landscape images. Mr Long's heritage of Miao descent and background as a painter meant that the walls of his office were lined with his paintings which we realised depicted similar villages and landscapes. Mr Long proposed that we develop a VCD project that incorporated Miao culture in some way. Very soon after this we met Hua, an interpreter, for the first time, and her husband Tim, who was a painting teacher. $\mathrm{Mr}$ Long organised for us to extend our trip to HNCU by one week to visit a Miao region in Western Hunan (Xiangxi).

We stayed in Fenghuang, also referred to as the phoenix ancient town, visited a number of outlying villages and the Shanjiang Miao Museum. Miao and Tujia minority tribes are said to populate the area, and we discovered that Tim was from the Tujia tribe. Fenghuang itself seemed a fascinating contradiction. Set on a river in a beautiful mountainous landscape, the mostly wooden houses jostled with busy karaoke (KTV) bars and restaurants. It was at the museum in one of the outlying villages that my colleague and I observed and photographed Miao artefacts and textiles. These objects displayed an abundance of stylised flowers, insects, animals and geometric shapes. Hua and Tim gave us a glimpse into mythical Miao stories, and although I did not know it at the time, Hua was to become my main interpreter for this research. In the car on the way back from this trip, the idea for a deck of playing cards was hatched during a conversation about the ubiquitous nature of card playing we had witnessed in the backs of small shops. Students could explore local and cultural motifs to inform a design for a deck of cards, and at the same time, we, and they, could learn more about Miao indigenous tribal stories.

Undertaking and teaching a cultural project such as this in China demanded a receptiveness to culturally appropriate ways of working. We were aware that intercultural awareness within transnational education and design pedagogy presents opportunities and challenges (Doyle, 2016). As we crossed cultural boundaries, sensitivities had to be navigated (Saris, 2018). Ensuring that the content for the project had relevance for the students involved a search for reference material that was appropriate, authentic and local. I undertook a literature review to understand more clearly not only the stories, but how to address my concerns around appropriations and transformations of historic and contemporary cultural identity and artefacts. I was 
deeply aware that we needed to avoid colonial pedagogical project critique, and wanted to develop an awareness and deep appreciation of local cultural stories and icons. Mr Long played a big part in the learning for the project. He prepared a lecture about the Miao indigenous tribal stories and became our cultural advisor in the classroom. He helped us to rethink and redefine content during the learning process as the project developed. It should be noted that the Saris (2018) article described the first teaching and learning delivery of this project which took place in May 2017, one year after our visit to Xiangxi. The research that was conducted in this pedagogical project was undertaken during the second 2018 delivery of the project. Both deliveries of this project fell within the duration of the $\mathrm{PhD}$ research.

\section{Choosing a topic for the Miao cultural project}

As previously noted, the decision to use playing cards as the format to display the motifs was made by my colleague and me during our visit to Xiangxi. There were two reasons that assured us that the project could not be seen as a form of imperialism (Yang, 2014b). First, we had observed the ubiquitous nature of card playing in the rear of small shops in Xiangxi, and tea shops in and around the environs of HNCU. We watched large groups gathered at tables on the lakeside in Yiyang at the weekends. The same suit and rank numbers as western playing cards meant the games of cards were familiar. Second, the literature speculates that playing cards originated from China and spread to the west in the form of early print samples (Lo, 2000).

\section{Miao teaching plan}

For the following discussion see Appendix 7.1., Miao illustration project: Playing cards (page 296). An explanation of the teaching curriculum structure for the project and the students' learning activities is evidenced by a number of photographs. As per the branding project, this appendix details the timings of the video recordings that took place for the $\mathrm{PhD}$ research.

In May 2018, I was scheduled to begin teaching the Miao project. The same teaching schedule as the previous branding project was stipulated, with classes conducted four days a week (32 hours contact a week for three weeks). Again, twelve four-hour lessons were devised for the project and to accommodate learning for both classes, this 
procedure was applied twice. I was assigned the same two classes as I had been assigned for the branding project. Therefore, the second time I stepped into their classrooms there was a level of familiarity with the students and their environment that was not evident the previous year.

For the Miao project a similar teaching plan as the branding project was devised whereby a DT process was followed. However, in the Miao project there was an attempt to integrate more dyadic activities into the learning. There had been five dyad interactions within the branding project, and there were seven incorporated into the Miao cultural project. This was not overly successful because students gravitated back to working individually much of the time, likely owing to the pressure of Chinese institutional rules mentioned earlier.

We began this project in a similar way to the branding project. To begin, the empathise and define stages of DT were combined into one four-hour class session similar to the branding project. In this class students were introduced to some of the Miao character depictions and stories associated with the various gods and animals through a lecture that Long Xiangping had prepared and delivered. After this a game of snap was played to randomly place the students into pairs to enable a sharing of concepts for one of the playing card characters. In the game, the first to snap a pair of cards wrote their name on the blackboard next to the corresponding card rank number. Once the pairs had been decided, students were encouraged to conduct research on the internet and visit the library to assemble reference images of their designated characters for a moodboard (Garner \& McDonagh-Philp, 2001). The aim of the mood-board was to assist in the DT ideate (idea generation) phase of the process which followed.

The ideate phase enabled 12 hours of experimentation over the next three classes. There was an attempt to encourage rigorous and 'manual' iterative processes in these classes where the aim was to use drawing, tracing, and cutting for idea generation rather than students relying solely on computer use. In these classes, students were less quick to jump on to their computers, perhaps due to previously restricted computer use in the branding project. A short prototype phase followed in class five where a colour palette was loaded into the swatches palette of the Illustrator software, and the colour 
applied to preliminary character designs. During the sixth class, another ideate phase was introduced. Students spent this four-hour block deconstructing and reconstructing the suits - heart, diamond, club, and spade - into new variations using black and red paper. The aim was to consider how the suit designs could be incorporated into character designs, or for aspects of the character designs to be integrated into a suit shape. This proved a successful strategy for some, as it led to a certain originality being nurtured.

Following this class, students were encouraged to keep applying an iterative process to their work within the prototype phases. I requested that the students print their work out and we would have class critique (peer review) sessions where all the students would stand facing the work. Systematically most were asked to respond to each other's work, and offer criticism and solutions. The students were reluctant to offer criticism, which I found especially interesting and explored further during the data gathering interviews. During these critique sessions, Miao stories were repeated, along with further suggestions related to zoomorphic, anthropomorphic, phyllomorphic, geometric and metaphoric approaches. From class nine onwards, I experienced a similar pattern of computer use as was experienced with the branding project. Once the students felt they had resolved one particular design direction and had set about building the design with the Illustrator software, they were very reluctant to revisit an earlier DT phase, change direction or change a colour. In fact, colour turned out to be quite problematic for the students. They challenged the colour palette that was stipulated for the class, preferring to use system colours. System colours are the automatic colour palettes that are linked to Illustrator when the software programme is first opened.

\section{Reflections and realities of the Miao Project}

The timing of this particular project was less challenging than the timing of the branding project. The students seemed more settled, and the milder temperature more conducive to learning. There were less distractions such as military training for the students and there were approximately six weeks to go before the long summer break would commence. Perhaps my familiarity with the students also contributed to a more settled learning experience for the students. Another contributing factor could have 
been greater involvement by the interpreters which is discussed later in this chapter. One of the interpreters I worked with during the Miao project included Hua who had accompanied us to Xiangxi. She endlessly discussed the stories with all of us through the learning journey. Mr Long also added his versions of the stories to the discussions and downloaded hundreds of reference images for the students onto our computers. It was a privilege to be granted access to his passion and personal image library compiled from photographs taken at museums throughout the Xiangxi and Guizhou regions over many years.

\subsection{Background to the HNCU teachers' interviews}

Towards the end of the data collection phase of the Miao cultural project, I was invited by a young female Chinese teacher to observe her freshman (first year) animation class. To my surprise she was instructing the students to faithfully copy a blue cartoon cat character. All of the students had to produce those characters in exactly the same way with Illustrator software (see figure 5.3).

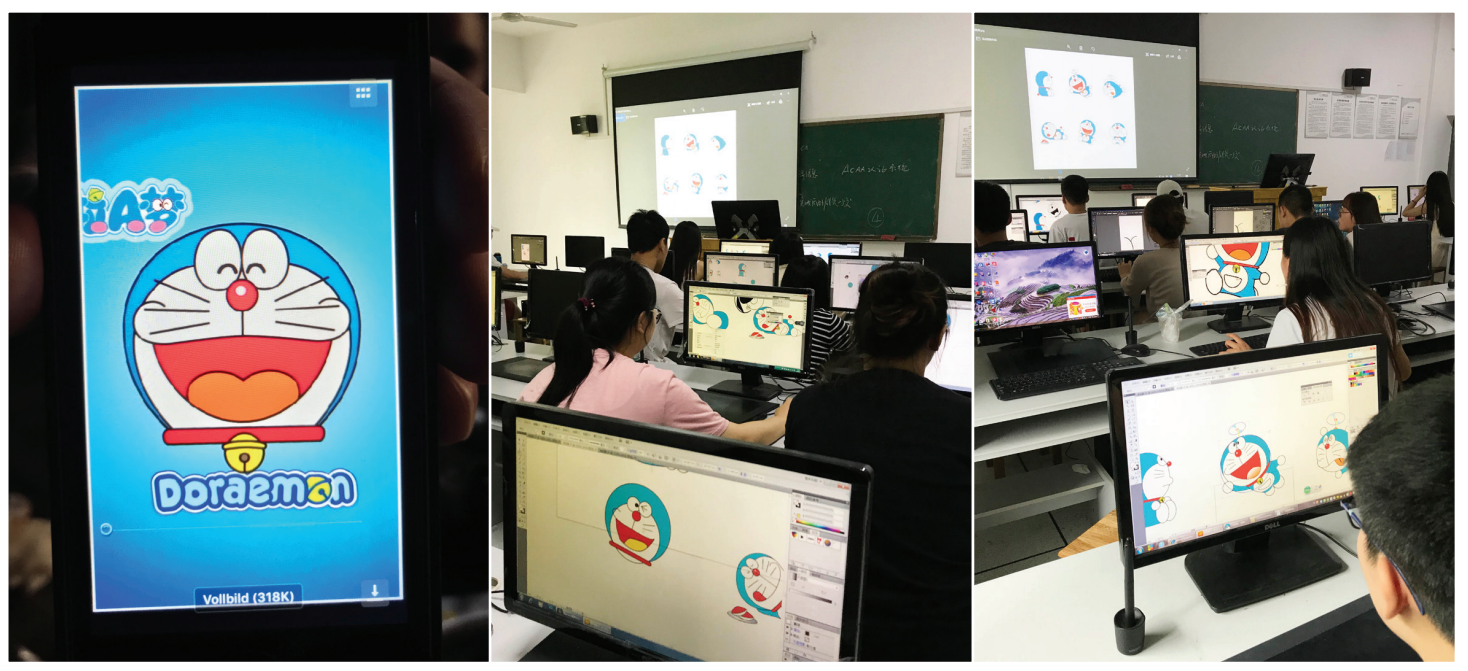

Figure 5.3. Animation class at HNCU (author images).

This learning approach resonated with some of the stereotypical literature discussed in Chapter 2, and my observations on that day helped in devising one of my subquestions - How do previous and current Chinese teaching and learning practices shape VCD creative design process learning? 
The opportunity to witness this learning approach deepened my curiosity about why this practice exists and I became curious to learn how these activities may enable or constrain learning. In certain respects, the reality of this process should not have been surprising for me. Sometimes in my own teaching I will instruct students to follow a worksheet to learn how to use the shape-builder tool for example, but this situation at HNCU presented a significant difference. Where I would elect to employ abstractions for these types of exercises, and encourage students' investigation of change in design outputs, I understood that these students were not permitted to deviate from replicating an exacting image of the blue cat given as a model. Furthermore, the obvious ease in which the students were working meant that arguably everyone's motives were aligned so the activity was focussed on the same outcomes, which appeared to include completion. The advantages of introducing students to such technical and observational skills cannot be discredited, and they in turn pointed to previous cultural learning experiences about which I had very limited understanding. Adding a layer of understanding about this practice to my research design through interviews with teachers had the potential to provide a richer and deeper account of creative design process learning.

My experience that day prompted a revision of the research design. As was previously discussed in the Methods chapter, findings from the two case studies were enriched with other perspectives that included eight semi-structured Chinese teacher interviews and observations in Chinese medium animation classes at HNCU and at an art training school for high school students in Changsha.

\subsection{Prior creative learning: addressing historicity}

As the case studies and teachers' semi-structured interviews progressed, the interpreters and teachers often referred to training schools. I was informed that all of the VCD students currently studying at HNCU would have spent time undergoing a rigorous arts programme at a training school in their last year of high school (grade three). This piqued my interest as it directly referenced Engeström's (2001) third CHAT principle, "historicity". Historicity potentially sheds light on problems against their own history, and it was argued that potential problems raised from problematic 
developmental and historic perspectives are often left out of CHAT studies (Moran \& John-Steiner, 2003). Therefore, as it was evident from my emerging case study findings that these training schools directly related to developmental learning at HNCU, I enquired into the possibility of visiting a training school. What followed was a fascinating glimpse into a day of high school arts education which took place at a training school in Changsha, the capital city of Hunan Province, approximately two hours' drive from Yiyang. In September 2018, a proprietor linked to HNCU took me to her privately owned training school. This was a small school which has been in operation for ten years. The school was situated in a cluster of other similar training schools in Changsha, many of which advertised their student works and class photographs on the exterior of the buildings (see Figure 5.4).

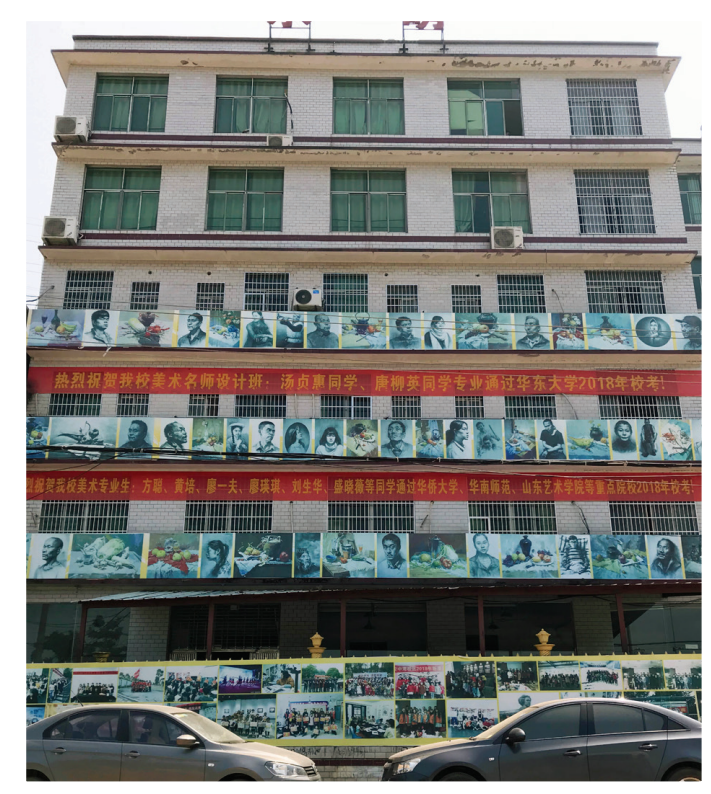

Figure 5.4. Exterior of a training school in the cluster of training schools, Changsha (author image).

The particular school I went to housed 100 grade three students from a high school outside of Changsha but still within the bounds of Hunan Province. The proprietor had an agreement with a high school to train school leavers for a nine-month period each year. The school provided segregated dormitory style accommodation (eight students per room sharing four bunk beds) and a canteen with a cook who supplied all meals. The classrooms were located on the ground floor of the main building in a small compound. The time allocated to learning was intense. Students were required to be at their easels by $8 \mathrm{am}$ every morning, with a lunch break scheduled at $11.30 \mathrm{am}$. At 
$2.30 \mathrm{pm}$ classes continued until $5.30 \mathrm{pm}$, when there was another scheduled break. The students would have dinner, rest, and then maybe play basketball. Classes resumed at $7 \mathrm{pm}$, and finished at $10 \mathrm{pm}$ when homework was set. I was told that often students would not complete their day until past midnight. This regime was followed every month, seven days a week. Students were allowed a complete break, three days a month only.

These grade three high school students were in their last year of high school, and this training school was their chance to enter a university of their choice if they succeeded in the 'arts exam'. As it was conveyed to me, there were approximately 20,000 arts students in Hunan Province in any given year preparing for the arts exam, and the Chinese examination system comprised two parts: the arts exam, and a liberal arts topic exam. The liberal arts topics covered subjects such as maths, Chinese language and history, English and politics. To prepare for the one-day arts examination, students had to learn techniques and skills for any of the unknown possibilities that were presented on the actual day of the examination. These possibilities were outlined as:

1. Portrait (face, face and shoulders, or drawing from the waist up) - 3 hours;

2. Speed [gestural] sketch (sometimes one person or a group, or a still life) - half an hour;

3. Colour (sometimes a still life or a landscape) - three hours.

On the Sunday that I visited the training school, I observed a portrait drawing class. The room was silent except for a teacher using a microphone to talk to blue uniformed students. Other teachers were moving through the rows of students, helping them with their work. The students were perched on small collapsible stools with drawing boards balanced on their knees. Using charcoal and pencils, students were faithfully copying a photograph clipped to boards in front of them (see Figure 5.5). 


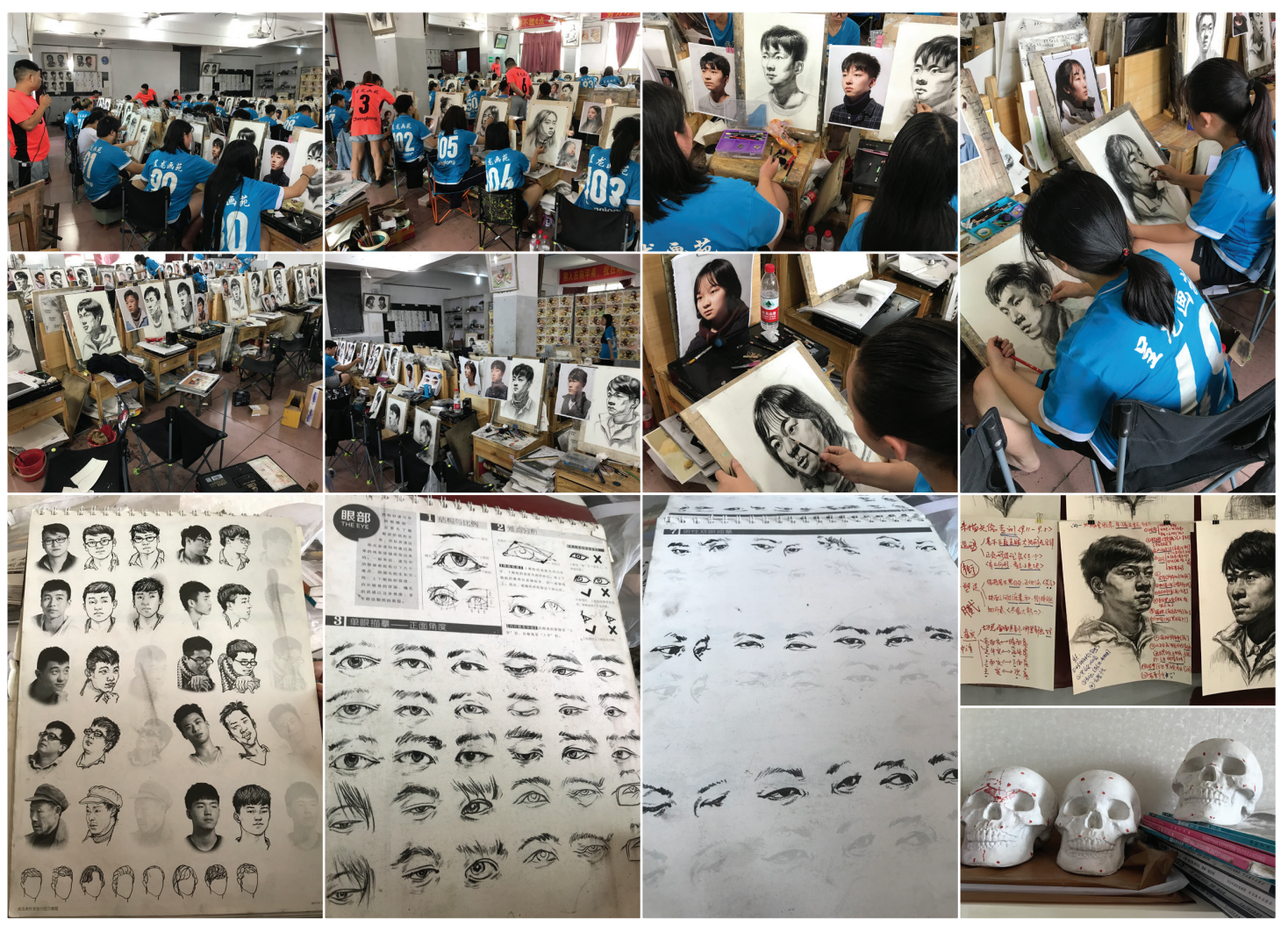

Figure 5.5. Portrait drawing at the training school in Changsha (author images).

To my 'foreign' eyes, this was astonishing, and as the proprietor laughed at my reaction, she said she had brought another 'foreigner' to her school and he commented that it was like a factory. Coupled with the information I had been given about the hours of learning and the looming pressure of the art examination, it was not difficult to recognise the enormous work ethic that was involved. Around the edges of the room, previous class work was pinned to walls and on the shelves, there were exercise flip books, and skulls that had been used by students (see Figure 5.5). The examples of flip books shown in this figure, outline a process whereby rows of heads or eyes begin with a photograph, and transition through to a light grey drawn image. Students could directly trace on top of these printed images. The flip books contained pages of other body parts, for example noses, hands and feet. Moreover, the shelves revealed large format books containing numerous photographic still life compositions (see Figure 5.6). This was the reference material for students to copy when practising for the colour requirements of the arts examination. Similarly, there were exercise books to support the gestural drawing learning. The coloured still life work and gestural 
sketches on the walls of the classrooms evidenced grades and sometimes intricate teacher comments (see Figure 5.6).

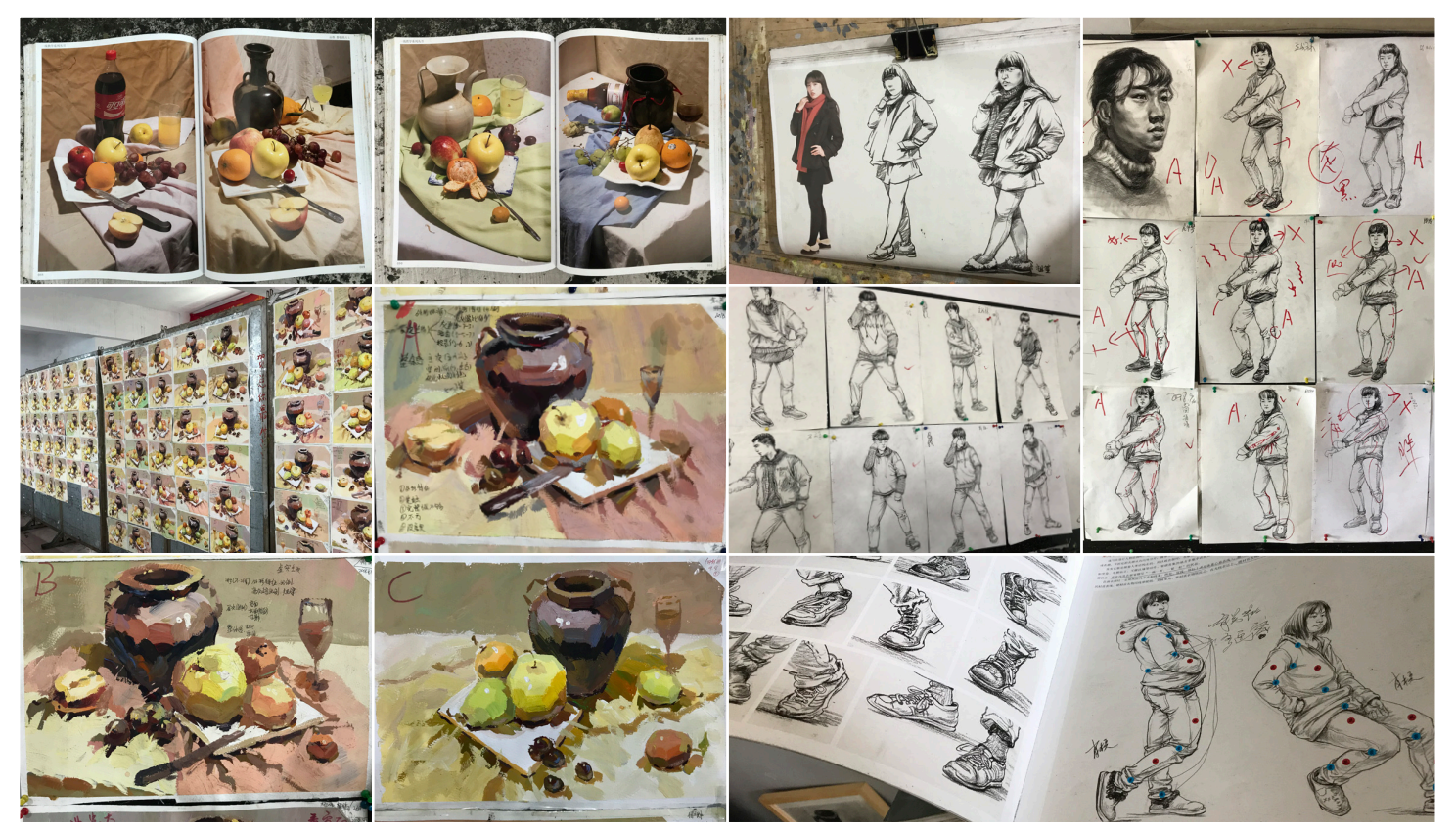

Figure 5.6. Still life, gestural reference material and student work (author images).

The trip to this training school was a welcome addition to my research as it enabled me to see through my own eyes how and why this prior intensive learning was problematic not only for VCD learning, but also for the Chinese teachers. The reason it seemed problematic was that the learning fundamentally encouraged a culture of direct copying. I observed teachers taking great care to teach the students minute details related to this, and the students responded by practising over and over to make their work perfect. There did not seem to be any room for alternative expressions, in-fact if the students deviated from the prescribed wisdom related to technique such as correct application of colour or brush stroke, they would fail. Fortuitously, the timing of the trip was helpful for some of the teachers' interviews as it occurred prior to their interviews being conducted. As a result of these observations my questions to the teacher participants became more focussed. 


\subsection{Working with an interpreter in the classroom and during the interviews}

To conclude this chapter, issues and revelations associated with working with interpreters are further elaborated (see page 90 for an earlier discussion). I came into contact with interpreters in two ways whilst conducting this PhD study, firstly, in the classroom, and secondly, during the interviews. Working with interpreters in the classroom was familiar; each time I travelled to China to teach, I was assigned a translator. The Chinese staff preferred to use this term rather than interpreter. When I taught the two projects this research is concerned with, I worked primarily with two women, Hua and Shing. They were both approximately 35 years old, full-time IELTS English teachers at HNCU. It was a privilege that these same two interpreters were assigned to the both the branding and Miao cultural projects because prior to these being conducted I had experienced issues when larger numbers of translators, up to six individuals, were assigned to my classes. Working with more than two translators meant issues related to consistency would arise. By consistency I mean communications were not conveyed in the same way in the two separate classes, thus the potential for student misunderstandings arose. With Hua and Shing I had previously formed a strong rapport prior to teaching the branding and Miao projects, as time had been spent with them outside classroom hours. We had been to the tea street together, visited cultural festivals and museums, and they had introduced me to 'foot washing', a form of foot and leg massage particular to Yiyang. When I had been researching the Miao project for the first teaching delivery I had travelled to Xiangxi with Hua. At the time of the delivery of the Miao project, Shing accompanied my colleague and me to an historic writer retreat and lotus gardens on the outskirts of Yiyang. We had many conversations discussing the finer points of the English language and Chinese history amongst other things. When I approached Hua and Shing to help with my interviews for this research, they both agreed without hesitation.

Inside the classroom, while I was teaching, Hua or Shing were constantly at my side. I had learnt that in conversing with students, short concise sentences enabled greater clarity of communication between myself, the student and interpreter. I also discovered that in order to temper my New Zealand accent, I had to concentrate on articulation which slowed my speech down. Owing to my familiarity with Hua and Shing, and 
their familiarity with me, we encountered few problems. Often, when I was repeating similar feedback to a student on a particular aspect of their work, for example correcting a software technique, Shing in particular quickly learnt the technique and would guide the students herself. She took great delight in 'learning design.' Hua became so involved with the teaching that she incorporated some of the research aspects of the classes into her IELTS classes which often followed my design classes. During a second delivery of the branding project which was conducted in September 2018, the year after the case study that this research is concerned with, Hua very proudly showed me work the students had completed in her class. She had spent her entire IELTS lesson guiding the students to help set the design goal for the project. They had worked together in the empathise/define DT phase to try to unpack problem formation and concept creation. Similarly, Shing, who was learning to become a Chinese tea ceremony master, instigated a tea-making demonstration. She arrived in class with a bag full of tea ceremony objects and proceeded to gather the students around a table to watch the intricate rituals. This work that both Hua and Shing instigated contributed greatly to the overall class learning (see Figure 5.7).

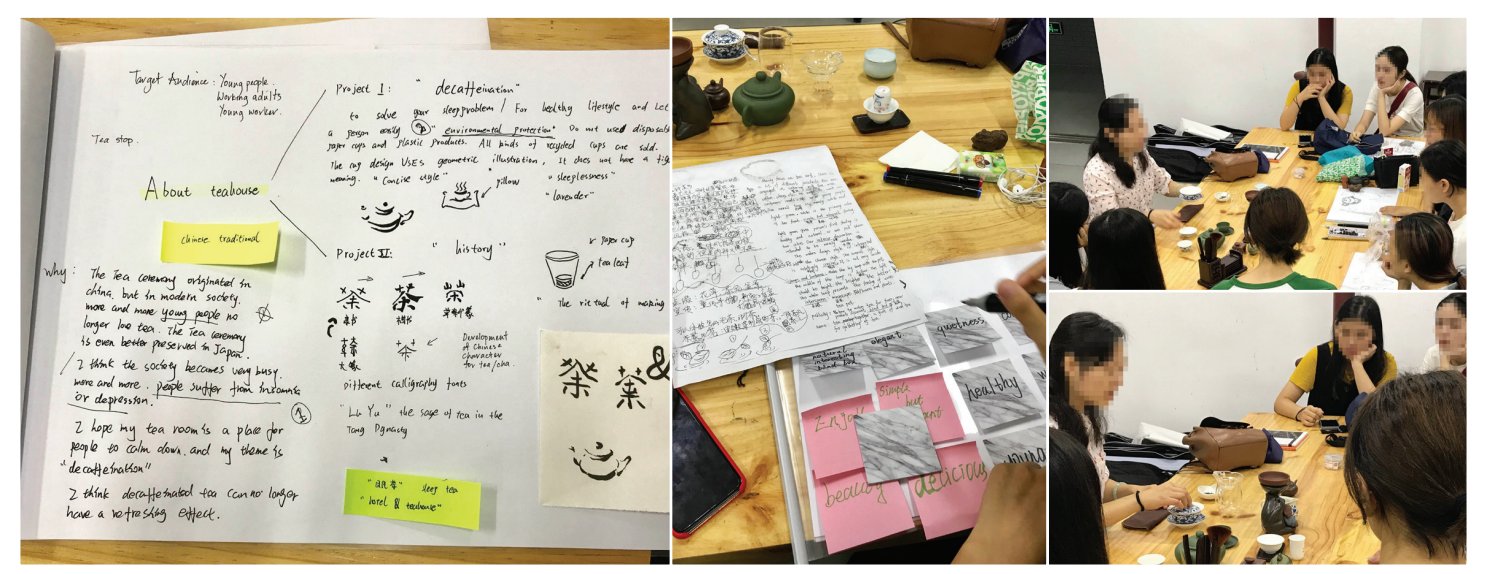

Figure 5.7. Student research instigated by Hua, and Shing's tea ceremony demonstration (author images).

Hua became the sole interpreter for the branding and Miao project student interviews. I did not question the trust we had in each other. However, there were some instances in the interviews where the student and Hua would engage in a long conversation, they might laugh, Hua would make notes and I would be delivered a short summary. For example: 
BS: That seems a lot for you to translate.

Interpreter: Yeah, but I think some of the points are very ...

Student participant: [in English] Detailed.

Interpreter: Not detailed, I think it's unnecessary some of the steps because he put more description on the process, I will translate it in outline.

The above excerpt highlights an instance of issues associated with working with interpreters during interviews and the richness of participants' experiences, and meanings potentially being misunderstood by researchers (Kosny et al., 2014). Despite this, had Hua remained my sole interpreter for all of the interviews, I doubt whether my confidence in her would have been challenged. However, concerns were raised about my reliance on Hua and her role in my cross-language and cross-cultural research when Shing became involved (Caretta, 2015). After the eight dyad (sixteen participant) interviews, I conducted eight interviews with individual Chinese teaching staff at HNCU. Shing interpreted three of these interviews. It was a considerable surprise to realise that Shing's candid and honest interpretations were different to the way Hua conveyed information. Some of what Shing interpreted was potentially contentious, for example she openly used the word 'copying' whereas Hua was more measured and used a softer 'imitating' when the teachers and I were discussing student activities related to this practice. The subtle nuance between these two words left me wondering how many of Hua's interpretations were being influenced by her own assumptions about the students, which in turn had the potential to result in biased interpretations by me (Caretta, 2015). Furthermore, the realisation that my personal subjective influences and interpersonal relations with Hua meant that my reactions and concerns about the students were potentially influencing what she was expressing (Williamson et al., 2011).

I had spent many more hours with Hua in my time in Yiyang than I had with Shing. Shing's involvement with the interviews brought another important dimension to the participant responses because her interpretations and voice were added to Hua's. Both interpreters became my key informants or instruments of inquiry, and as such, their involvement with me in social situations inside and outside the classroom meant they had influence in my research (Edwards, 1998; Merriam, 2009). Therefore, ethically 
and methodologically it was my responsibility to manage this as was previously discussed on page 90 .

\subsection{Conclusion}

Critical to activity theory is the way in which context interrelates within activity systems. Therefore, for the analysis it was necessary to explore the complexity of interactions and relationships to the wider social, cultural and historic contexts of the research. In particular, when determining objects (units of analysis) of the participant activity systems, it became clear that contextual influences were mediating activities in ways that were both seen and unseen by me (Engeström \& Miettinen, 1999). From the more tangible contexts a picture of study site has been provided, and the teaching and learning timetables and overview and pedagogical underpinnings for two pedagogical projects have been presented. The background context relating specifically to the branding and Miao cultural courses has been provided. Course curriculum was designed specifically to accord students with cultural learning contexts that were relevant and familiar. Vibrant cultural experiences inside and outside the data collection phases contributed to the pedagogy of both of these courses, and within this my perspectives and ways of working with students at HNCU were all the more richer (Geertz, 1973; Merriam, 2009). Further elaboration and the analysis of the two projects follow in chapters 6 and 7 respectively. The analysis of the teachers' interviews follows in chapter 8 .

The inclusion of the teachers' interviews and observations undertaken in Chinese medium classes revealed aspects of student learning that may have remained behind closed classroom doors. Having the opportunity to observe Chinese teaching and learning methods first hand has resulted in findings laden with the "crucial nature of context" which was pivotal in establishing meaning about creativity and creative design process learning at HNCU (Guba, 1987, p. 28). Significantly, understanding meaning was enhanced by addressing "historicity" (Engeström, 2001, p. 136). Visiting the art training school in Changsha was a surprising experience for me. The teaching and learning being conducted there was undoubtedly recognisable as a problem for VCD education from a New Zealand perspective, and at the time I found it difficult to 
ascertain what the potential of the activities could be (Engeström, 2001). However, this experience made it clear that the VCD students' creative design process learning at HNCU had to be analysed against both these historic high school level practices alongside current pedagogical practices shaped by both Chinese and New Zealand teachers. Context, in its various guises, has provided sources of tension and contradiction which played a role in change and developmental learning (Engeström, 2001). To explain this more, I begin with the branding case study analysis which follows. 


\section{Chapter 6. Branding case study}

In the Chinese VCD learning context, teaching innovation, creativity and the creative design process are congruent with policy influences from the Made in China 2025 strategy. Implemented by the China State Council in 2015, the ten-year strategic plan aims to move China's manufacturing industry to an innovation industry by positioning strategic design at the centre of business practice (Liu et al., 2018). Branding is a key component of this vision owing to the role it plays in marketing and visual communication. The case study in this chapter used a pedagogical branding project where the focus of the learning was to encourage an iterative process within the design thinking ideation and prototype phases. A fictional Chinese tea business was used as a basis for students to design and develop visual identity elements for a new tea brand, previously discussed in Chapter 5. The focus of the analysis is to tease out how students navigated a different pedagogical approach by uncovering tensions and contractions that were experienced by four VCD student dyadic AS. The four dyads comprised eight sophomore individual student participants who executed creative work in the project. The project and research were conducted within a three-week period, at Hunan City University (HNCU) in China, where I was timetabled to teach two classes. Participants for dyads one and two were recruited from class number 203, and, participants for dyads three and four were recruited from class number 205 (see Figure 6.1).
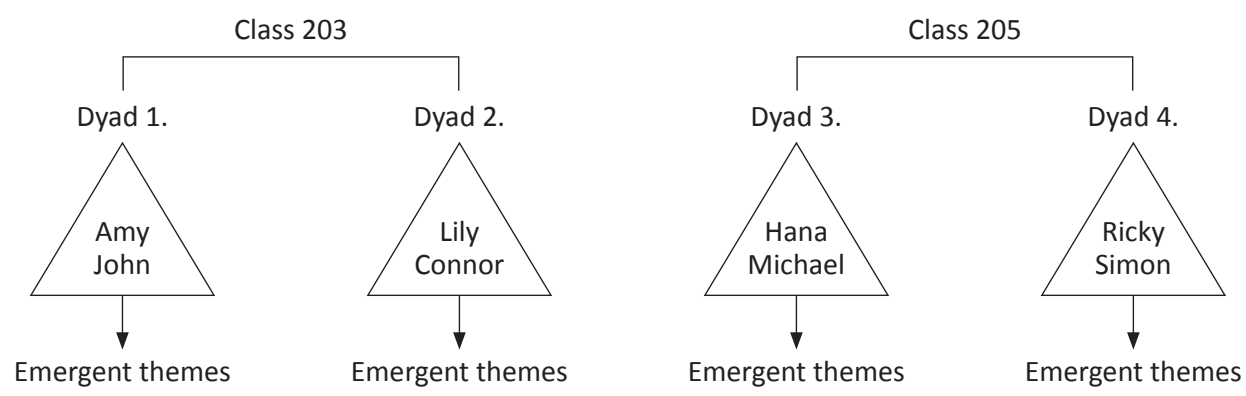

Figure 6.1. Case study one: Pedagogical branding project. Four dyadic activity systems. 
Figure 6.1 details pseudonyms used in place of actual student names. The class of 203 (25 students) together elected to work on the brand name tea friends and 205 (26 students) decided to work on Tone/1.

Insights and themes from an analysis of the four separate dyads focused primarily on the inter-related interplay of primary, secondary, and tertiary artefacts. In the threelevel hierarchy of artefacts that was constructed for the analysis, primary artefacts are those tools, traditional and technological, which were used unconsciously in basic operations and VCD production, for example, a pencil or computer. Secondary artefacts are those tools used for transmitting action, for example, the hand in praxis, or discursive constructs - subjective and emotional factors which influence the deployment of primary artefacts. Tertiary artefacts are those tools which account for processes of the mind in design process, or abstractions which involve possible or imaginary worlds, for example using the mind to envisage how colour could be used to change a scene from being gloomy to bright. It is important to note that although the three-level hierarchy of artefacts were separated for the analysis and discussion, they constantly interrelate with both the subject and object within a AS (Foot, 2014).

As outlined in Chapter 3, the analysis approach used for the research, was based on Leont'ev's theorisation of a three-level model of activity theory (Engeström \& Miettinen, 1999), Foot's (2014) ideas about the characteristics of tensions and contradictions occurring at the top, middle and base levels of an AS, and Engeström's (1993) assertions that inner contradictions take into account wider contexts of an activity system. When object-oriented motive/s drive interactions between the subjectartefact-object at the top level of an AS, constant tensions and contradictions are exposed by this this particular relational aspect of the analysis (Foot 2014). To recap, top level tensions relate to use and exchange value whereby a participant may directly benefit from an individual action, which means collective activities may become worth something during a division of labour exchange. To round out the analysis of each dyadic activity system, emergent findings are presented from the middle and base levels of the AS. The middle level evidences tensions and contractions when two AS nodes come into conflict and is the part of an AS whereby subjects are focused on goals or outcomes. The base level is the operational part of an AS where actions and 
activities are guided by conditions, rules, community influences and division of labour (DOL) interactions (Foot, 2014). All three levels are cyclical meaning they correspond to a particular developmental learning action(s) sequence. For example, at the top level, students may question, criticise or reject aspects of existing practice, at the middle level, students may analyse and implement a new model of learning, and at the base level contradictions may result in the consolidation of a new practice or process in learning (Foot, 2014). In support of the dyad findings, additional data are included from 100 student reflections which were collected from all students who participated in the pedagogical project. Inclusion of the written reflection analysis added to the picture of the dyadic creative design process.

To conclude this chapter, the four dyadic AS are situated within an overall activity system of the class project for discussion. I now present the analysis of the first dyad activity system, Amy and John.

\subsection{Analysis}

\section{Dyad 1: Amy and John}

My field notes described Amy and John as a quiet pair, and that their interactions with each other appeared considerate and respectful. Amy seemed to take the lead in their design activities by being more vocal in their conversations, whilst John was observed to contribute to the DOL in a less demonstrative but thoughtful way.

\section{Theme one (1): Managing the effects of primary, secondary and tertiary artefact tensions}

The first key theme to emerge from the analysis reflected tensions that arose from the interrelated deployment of primary, secondary and tertiary artefacts within the AS. Identified as managing the effects of primary, secondary and tertiary artefact tensions, the theme highlighted the way object-oriented motives were used by the dyad. One of the conceptual directions Amy and John briefly explored as they worked on the brand name tea friends, touched on emoticons or smiley faces, which are popular culture 
symbols used to express facial expressions in place of a word. These are commonly used on WeChat, the ubiquitous social media application used in China. Two technological primary artefacts, their mobile phones and the internet, were used to access representations of emoticons. The mediating role of the mobile phone and internet search, along with their exchange of opinions and viewpoints about this particular conceptual direction, resulted in a decision by the dyad not to progress the idea. They next moved on to consider another imaginative conceptual direction which attempted to combine the yin yang cultural sign relating to Chinese philosophy and tea friends into a kind of synthesis. The excerpt that follows identifies learning actions in which the dyad questioned their ideas, and the way the use and exchange value mediated artefact use. The role of imagination (tertiary artefact), exchange of opinion and viewpoints (secondary discursive artefact), an internet search using a computer (primary technological artefact), and a pencil (traditional primary artefact) resulted in a sketch that expressed an imaginative concept:

Interpreter, translating Amy and John: So, first they had two ideas, the first one is when they got tea friends so they relate friends with two different people, different characters can work together, so that's just like a symbol, yin yang, different people can work together. And they had another idea that is from the facial expressions that we have on WeChat.

Brenda Saris (BS): Do you mean the emoticons, the smiley faces?

Interpreter, translating Amy and John: Yeah, but they think it's not serious to use that kind of symbol, so they focus on the yin yang symbol, and at the beginning, so first, they thought of the yin yang fish . . but they don't think it's good, so they make some change, so they make the sketches.

BS: How did you make those changes?

Interpreter, translating John: They searched the internet and they found some pictures made by the shape of fish, and fish stands for lucky.

These insights led me to ask them where they thought their creative ideas came from:

Interpreter, translating Amy: It's an idea, popped up in the head, and in the mind.

Amy immediately referenced an a-ha (illumination or design epiphany) moment when she responded to the question. Her acknowledgement of ideas popping up in the head 
and in the mind means an unexplainable process, took place in her mind. However, whilst discussing the circular yin yang symbol, the dyad revealed their struggle to progress the idea:

Interpreter, translating Amy: Once they get an idea, it's very hard for them to get another way to think, for example, this shape is a circle [referred to the work on the computer screen], so, it's very hard for them to think of something which is not a circle anymore. It's an idea, popped up in the head, and in the mind.

Interpreter, translating John: It's very hard to get new ideas once they fix on the circle design.

I asked Amy and John why they thought the circle design was a good idea:

Interpreter, translating John: So, from the start they thought that this circle is a typical Chinese element, so they start from this circle, then [laughs] it cannot jump out.

Thus, the dyad referenced a design block tension related to tertiary artefact abstractions and it was interesting to note that they began with a cultural idea. To explain this, Amy and John's abstract concept involving imaginary circles stalled their creative process and their imagination became blocked. Despite these challenges, a few days later during the prototype design thinking phase, Amy and John tended to work individually. While John worked on one of the other directions the pair had conceptualised during the ideate phase, Amy chose to progress the circular concept through a deconstruction of the black and white yin yang component parts. She experimented by using the traditional yin yang shapes to represent water droplets from nature and placed the Chinese character letter forms for tea friends inside the shapes. The components were put into a circular device which could be construed to represent people sitting at a round table in a typical social situation in China. Arguably, the AS top-level tension relating to the circle and the design block, which I previously discussed as a contradiction, was overcome. The initial design block experienced by Amy and John became beneficial for Amy's final design. The circles provided scope for embedding further meaning in her design, and so Foot's (2014) interpretation of a top-level tension being beneficial was realised. 


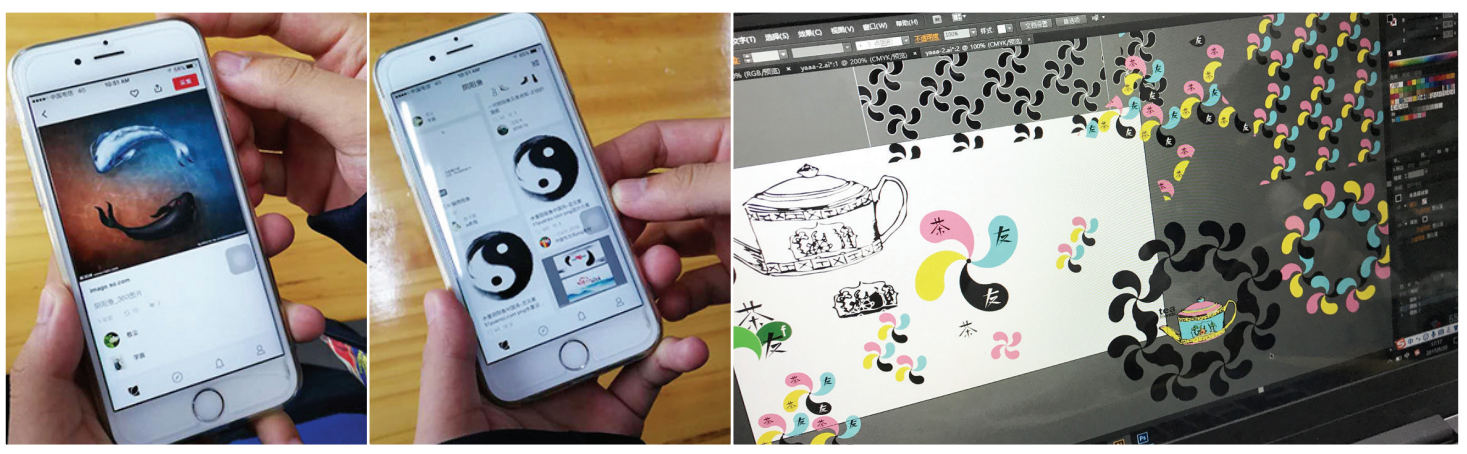

Figure 6.2. Amy's stages of design development referencing Chinese cultural elements (author images).

Although Figure 6.2 indicates that an iterative process was undertaken, there was conflict between my motive, which was to teach iterative processes, and Amy's motive which appeared focused on completion. In the interview, Amy indicated that she was disappointed her first draft was not accepted, but was quick to say that she was able to adjust her emotional state which indicates that emotional adjustment factors were in play:

BS: I'm going to show you this Amy, this is for you. There was a point where I came over, and you explained your idea to me and then you smiled here [wry smile], so can you describe for me, were you satisfied with the feedback that you were getting or were you not satisfied? Can you tell me how you felt?

Interpreter, translating Amy: If her first draft cannot be accepted, so, she almost felt a little disappointed but after that she would adjust her mood, and do it again.

BS: How easy was it to adjust your mood?

Interpreter, translating Amy: So, it's easy for her to adjust her mood to do it again.

One of the 100 student reflections (SR) that had been collected echoed Amy's point about mood:

SR23: You need to be open minded and have a good mood to make your design. And don't forget your patience.

Even though Amy said she was able to adjust her mood, both Amy and John found it challenging to undertake an iterative process. The dyad eliminated two of their first conceptual ideas, the emoticons (happy or sad faces constructed by keyboard 
characters) and fish, without any further exploration. Their initial resistance to engage in DT ideation processes which rely on iterative practices was being taught by me in the class. The rule that was imposed on the learning was that the students should produce several concepts, but the number was unspecified; however, there was an expectation that the students would explore a number of options. The articulation of this was conveyed to the students by the interpreters working with me in the classroom. Amy highlights that she was more interested in designing a beautiful logo. Both participants would have preferred the idea that they should adhere to a strict linear pathway which focused on a goal of completion. In fact, Amy was almost fearful of deviating from such a path. Fear may be interpreted as an emotional secondary artefact in this context. Moreover, it can be argued that a product over process motive was more important to the dyad than a recognition of serendipity leading to new or different conceptual ideas. Furthermore, the focus on goals, indicates that a middle level AS tension was encountered by the dyad within this theme:

BS: How might you have done things differently Amy?

Interpreter, translating Amy: So because for the first few lessons Amy didn't put emphasis on the connection between the meaning that she wants to convey.

At this point Amy was referring to the mind map brain storming exercise which had been introduced into the learning. This exercise was used in the ideate DT phase to help the students to determine a brand value or the essence of their tea brand in order to establish a design concept. Post-it notes were used to write down and to define what kinds of emotional feelings or tea attributes could be used to help articulate a brand direction, for example, happy, relaxing, healthy, fragrant:

Interpreter, translating Amy: At that time she only know that she wanted to design a beautiful logo without thinking much of the emotional side, because you said that branding is a kind of expressing the emotion, so if do it again she would more emphasis on the emotional side, the meaning with the logo to combine them together.

Interpreter, translating Amy: So, what is most important for her is to fix the theme first, the main idea, the theme first, and then she can stick to the theme, to work on it, because if sometimes she did something on the computer she very easily to go the other side, not focusing on the theme. 
BS: What about you John, can tell me how you managed the process?

Interpreter, translating John: So almost the same ideas, so he thinks that first should set the goal and to know very clear what to do next.

There were a number of student reflections that supported Amy and John's difficulties with using an iterative process. The writing ranged from fun-like descriptions or conveyed a real sense of dismay:

SR52: A designer must have a clear idea about the project ... in fact, inspirations and you begin a game of hide-and-seek. It is a little naughty boy which has many face that you did not see ever before;

SR54: There were a lot of troubles, such as frequent disorientation and loss, and many times I felt what I designed was not as good as I thought.

However, within the student reflections, there were a number that contradicted Amy and John's approach, with some reporting they had benefitted from the taught iterative process:

SR13: The most profound impact on me is to expand my thinking model, and its bold creative ideas;

SR24: The design process has eliminated some undesirable works and finally a good logo was designed. I'm very happy!

SR32: Let me deeply realise the fixed thinking is unable to make good design! When I first started the first logo ... after continuous attempts, breakthrough, innovation, only the last logo design, let me know after repeated attempts the new breakthrough, in order to select a better design;

SR58: My thought is diverging ... I see things not so limited, but there are also some regrets ... I don't open my mind;

SR81: This is a difficult and time-consuming process. I must keep trying and keep changing, keep comparing and keep checking, until the best comes along. Though it is a lengthy process, but it's not boring, I love my design;

SR97: I have learned to open my mind to innovation when I never tried.

Moreover, for an iterative process to flourish, it became apparent that teacher involvement was sought and valued by a large number of students. Students tended to wait for an approval from the teacher before moving on to the next stage, which 
indicates a lack of individual readiness to initiate further action within the design process. The following excerpt reveals aspects of this, for example:

SR09: I successfully carried out my own logo design with the help of my teacher. During this period of study I lived a full and happy life;

SR37: Maybe design needs more thinking and crazy. But I'm timid in design. Terrible! What's more, I must say thanks to you. Because you always help us patient. Maybe you think, oh, it's terrible! It's ugly!! But you will say it's ok! The idea is good! It's a very wonderful experience for me;

SR61: My teachers help improved my work ... of course I have some shortcomings, not creative enough, not enough ideas, makes the formation of the work slow;

SR64: She encouraged us to constantly try to combine new things to enrich our design;

SR66: The teacher's unconventional thinking had given me endless enlightenment;

SR78: The teachers reverse thinking, open up new thinking makes me wonder how much magic can be produced by the brain.

\section{Theme two (2): Adjusting to collaborative learning approaches}

The reflections above, and responses from the dyad below within the DOL revealed that most students were positively inclined to include the teacher as well as their classmates in collaborative learning approaches. The title of this theme attempts to capture the adjustment that was required with collaborative approaches. Both John and Amy talked about the benefits of combining ideas to ensure all avenues of experimentation were explored. The way the word negotiation was used by Amy suggested a genuine acceptance towards this way of working. John accepted Amy's ideas around negotiation by expressing his reluctance to push his ideas on to the other person. A shared DOL in the activities was found to operate between the participants:

BS: Can you tell me, how did you find working together?

Interpreter, translating Amy: It's a kind of negotiation between the two, they have their own ideas, then if they want to do a design by themselves they have to negotiate the ideas from each other and get a combined idea. 
Interpreter, translating John: So he cannot put his ideas, to push his ideas to the other person so they have to take others' ideas into consideration.

BS: How did that make you feel?

Interpreter, translating John: He thinks it's good to have other suggestions to help him to build a better design.

Issues arose for Amy when John was unavailable to support her ideas. She said she relied on other students in the classroom for a conceptual overview of her work when she became too focused on details of her design. Moreover, the evidence suggests that when she experienced a design block, or restriction of imagination, thus referencing both tertiary artefacts (process and abstractions), she sought out ideas and concepts from her classmates. Amy then tried to work out how to attribute these as her own. Using others' ideas and concepts is potentially different to a fluid exchange of ideas and concepts. Being inspired by others and bouncing ideas backward and forward can be viewed as transformative because ideas can be pushed along more quickly. Directly using others' ideas raises the question, who is doing what work? The interesting thing about this finding is that a middle level AS tension was encountered. Amy's learning actions were focussed on her goal, which was to find a way through the design block, and so by analysing the situation, she tried implementing new models or ways of doing that drew on her classmates' activities. In her evaluation of this process she revealed that the help she received in the form of critique and conceptual overview was beneficial:

BS: My question is around how you worked with other people. I know that once you stopped working together, you went back to your places and you worked next to other people. So how helpful were your classmates with your ideas, for your ideas?

Interpreter, translating Amy: For one point, because she works before the computer for a long time and she focussed on the details, and at that time another classmate came, and give her some ideas for the overall, give her overall view, and so the suggestions is very helpful because it is not focussed on that one point. It is overall, the general picture.

BS: And how was that was helpful?

Interpreter, translating Amy: She thinks it's very helpful, because they can spot the overview. 
BS: The big picture?

Interpreter: Yeah, yeah.

BS: Did that happen often?

Interpreter, translating Amy: Because she is so involved in her work she cannot spot the weaknesses in her design, the other students can give her the overview, so it's very helpful for her.

Interpreter, translating Amy: By looking at other students' designs it is a good way to exchange the ideas to get inspiration from others because when she saw some designs from other students, she wants to try the same, in a similar way and change, to turn the idea to herself. Turn the idea to hers, not to herself!

Student reflections support these findings, for example:

SR16: Through the design of other students I found the difference between the others and me, I'm still not good enough;

SR36: When there is no inspiration we can go and see someone else's design;

SR71: If you have difficulties, you need to be patient and solve them step by step. When you can't, stand up, walk, relax, or ask the other students for advice.

Surprisingly, during the discussion around collaboration the issue of the international English language testing system (IELTS) was raised. According to the dyad, this was the only class where students cooperated with each other to practise language skills:

BS: Do you work in pairs a lot, is this unusual for you or is it common?

Amy: [In English] Sometimes.

BS: Sometimes [interpreter laughs], can you explain how many times?

Interpreter, translating Amy and John: So, they think it's not usual, it's not usual to work in pairs, except the IELTS speaking class . . . in IELTS speaking class they cooperate with each other in pairs, to practise, while in other class, in other design classes, they do not co-operate usually.

This concludes the analysis and discussion of findings from the first dyadic AS.

The naming of the themes just discussed relate to two nodes of the AS - mediating 
artefacts and division of labour - and from these themes top and middle level tensions were revealed. An important question emerged from the middle level tension found within the second theme which was connected to the DOL. Who is doing what work? This question arose through Amy's discussion about using and being inspired by others' ideas and concepts, and so to a certain extent may be interpreted in two ways. This classroom practice in which an individual action draws on the activities of others, may or may not result in a beneficial outcome. A further middle level tension was found where secondary artefact (fear) and rules (iterative process) were imposed on the learning. This finding did not result in the naming of a theme at this point; rather, it was directly attributed to a motive, product over process owing to the prevalence of these issues being discussed, and the focus on completion of visual identity elements for the branding project. Figure 6.3 depicts the findings from Amy and John.

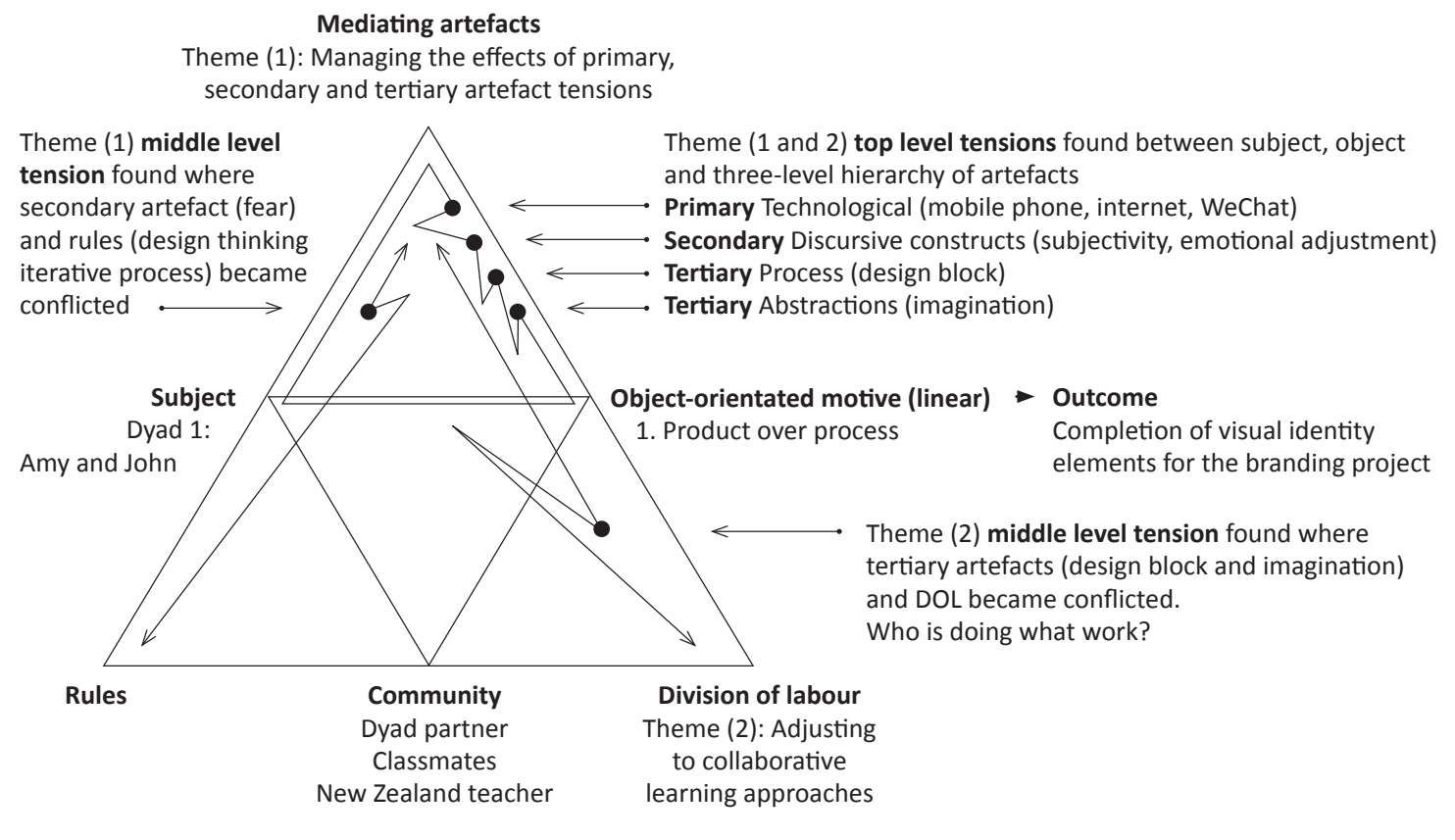

Figure 6.3. Dyad 1: Amy and John, themes and tensions.

The convention of using jagged lines to represent tensions and contradictions within an AS that many researchers appear to use was drawn from Engeström (2001). The lightning bolt effect with the arrow heads denotes the nodes of an AS which are implicated in the tension /contradiction, for example rules and mediating artefacts. The positioning of the dots on the jagged line depicts the level within the AS in which the 
tension or contradiction occurs; in the figure just presented, four top level tensions were found between subject, object and the three-level hierarchy of artefacts, and two middle level tensions were found. The first middle level tension depicts the conflict between a secondary artefact (fear) and rules (design thinking iterative process), and the second middle level tension depicts the conflict between tertiary artefacts (design block and imagination) and the DOL.

\section{Dyad 2: Lily and Connor}

Lily identified as one of the few minority tribe students in the class; she said she was from the Manchu minority and that her grandparents came from the LiaoNing province. At the time Lily told me this, she pointed to Amy and said she was also minority. Amy told me she was from the Hui minority, and that she came from Urumqi which is the far western province of Xinjiang in China. The reason I mention this is because I found it intriguing that from the overall majority of Han students, these two girls were the only minority representatives, and both had volunteered to be participants in my research. Connor on the other hand was a very sophisticated Han student. He had travelled outside China and his English language skills were more advanced than most of his classmates. He had a love of black and white photography, and usually came to class with his camera around his neck. The interactions between Lily and Connor were slightly awkward to begin with, but Connor said to me he was happy to work with Lily because she was very pretty. I'm not entirely sure Lily felt the same way about Connor, preferring to talk with her girlfriends whenever the opportunity arose. Lily was also the class monitor for class 203 and as such, was popular.

Within Lily and Connor's activity system, managing the effects of primary, secondary and tertiary artefact tensions was found to recur as the first theme. Again, the entire three-level hierarchy of artefacts was found to be implicated and tensions were driving object-oriented motives. An excerpt from Lily's transcript highlighted constant toplevel tensions that circulated between the subject (Lily), object (Lily's motive), and mediating artefacts (primary, secondary and tertiary). Specifically, the primary tool referred to in the excerpt may be read as computer software, secondary artefacts 
include praxis (drawing) and discursive constructs (subjective lack of confidence), and the tertiary artefact in question here was imagination (the idea in her mind):

Interpreter translating Lily: The biggest problem for her is how to use the computer to use the software to express what she wants to express. Usually she can have an idea in her mind, and she can draw it very beautifully, but she cannot use the software to express it. And, the other point is that Lily thinks that, she thinks she is not that capable to design very excellent work.

Explicit was Lily's frustration at being able to imagine and draw a beautiful image, but lack of software skills and a subjective lack of confidence thwarted efforts to produce a satisfactory outcome. Additionally, although it appeared Lily's motive was to produce excellence, her lack of confidence was also related to working with Connor:

Interpreter translating Lily: Connor is very talented as I said, so Lily felt a little bit stressful, so she's a little restrained in designing with Connor.

Thus, Lily's negative opinion around her design abilities had the potential to restrict transmitted action. Issues with deployment of software was not only problematic for Lily, but for the wider cohort group. None of the students used licenced design software. All used legally questionable versions of a huge array of different laptops with the more unique distinction being that many different operating systems were in play. On further investigation, it was discovered that students are expected to fund their own hardware and software, and that the university did not provide any technical support.

Following Lily's attestation involving a subjective lack of confidence, Connor mentioned an additional secondary discursive construct, laziness:

Interpreter translating Connor: Actually, for Connor, so for him [Connor: interjects. Lazy, laughs], he thinks that the biggest problem at college is a personality. A kind of laziness, because at college he is how do you say, he cannot allocate enough time to designing and the most of time after, after class he relax while he can only use the class time to do the design. So, the class time is very limited. This is the cause of the laziness. 
This candid acknowledgment of laziness suggested that Connor's personal life took precedence over his university life. There was a hint that Connor could be suggesting a level of boredom, ambivalence, or the subject matter of the project was unstimulating. However, the mention of needing to relax should not be underestimated for these VCD students. Timetabled classes began at 8:00am sharp, and the day ended at 6:10pm, with one- or two-night classes scheduled most weeks; it is unusual for hours lost to public holidays to be made up during the weekend. Therefore, interpretations of Connor's laziness could be linked to tiredness as it was not uncommon to observe students falling asleep in class.

A tertiary artefact involving an important cultural value and creative process aspects was referenced by Lily. She expressed a desire to ensure a more perfect start and arrive at a perfect final design if she could repeat the pedagogical activity. This meaning of perfect relates to something being faultless through actions or process of improvement which is suggestive of an inclination to produce a single outcome, thus the notion of product over process in combination with perfection is evidenced. Lily had a clear picture in her mind of how she envisaged her outcome rather than allowing a process to guide the result:

Interpreter translating Lily: If she do it again she would like to prepare more carefully because the design that she has now is not the one she intended to get. So, she wants to get a more perfect start and then work on it to be a perfect final design.

Connor expressed a similar ambition, but the link to perfection can be interpreted differently. The next excerpt links perfection to an unstated cultural rule or motive:

Interpreter translating Connor: If given a chance he can do it again, he'd like to make the design to be simpler, and ... [Connor interrupts the interpreter, and speaks in English]. Just like the 'apple' idea. To be very simple, only use very simple shape, for example a shape of a leaf.

Connor's aspirations to use simplicity to create a simple apple-like logo referenced the Steve Jobs apple brand which had been discussed in class. His desire to try to use simplicity may be interpreted as a contradiction that was encountered during the class. To explain, Connor's motive of trying to achieve simplicity may be indicative of a 
deeper level of cultural exchange and understanding emerging, with connotations extending beyond the classroom. By this I mean the apple logo may be a simple logo due to its depiction, but interpretations of the logo (sign) are more complex. The sign embodies brand values which encompass highly innovative and technological advancements, and these may have been understood by Connor. Therefore, his imagination had not enabled him to realise his motive which was to create a logo that was as perfect or as multi-dimensional as the apple logo.

In an example of trying to overcome or manage artefact tensions, Connor was observed in the video footage filling his sketchbook with large painted calligraphic marks. When asked to explain why he decided that making these marks might be a better idea than making conceptual drawings with pencil and paper which had been the class directive, Connor had difficulty explaining his actions. His determination to explain resulted in his words bouncing backwards and forwards between himself and the interpreter:

\footnotetext{
Connor: It's totally different ... [reverts to Chinese] Interpreter, translating Connor: Because he learned Chinese calligraphy for some time and the lines is totally different with the brush.

Connor: When we use the Chinese brush we can have many, many totally different shapes ... [reverts to Chinese] Interpreter, translating Connor: Because we can find some Chinese elements in the Chinese calligraphy because the strokes are typical Chinese.

Interpreter, translating Connor: He was wanting to get some ideas to get some feeling by writing the Chinese calligraphy.
}

Two things are referenced: first, tacit learning Connor could not explain his actions by the way he tried to use different words to help me understand why he chose to introduce calligraphy to his process; second, the introduction of calligraphy in prior learning can be interpreted as introducing an object of a more advanced cultural activity to the system. This is contentious given the action that calligraphy uses the hand in praxis, and with that, the implicit touch experience of mark making with a brush on paper, and so Connor was referencing a secondary artefact. However, his 
explanation about trying to find typical Chinese elements in the strokes, and the interpreter's use of the word feeling later on in the discussion has led to my interpretation of Connor's act of introducing this cultural activity as an attempt to trigger developmental learning and thus spark his creative process. This base level contradiction with its inherent potential for innovation was therefore illuminated within the activity system. To explain my interpretation of this base level contradiction further, Connor was guided by the rules of the class, and so his learning actions involved the evaluation and questioning of his process. His object-oriented motives drove his attempts to consolidate a new practice through the use of calligraphy which was a process that drew on previous learning, and in which other experiences, for example, other forms of teaching, were present. This in itself draws on Engeström's interpretation of object. It also provides an example of Foot's (2014) three facets of the problem space: first, an object is "a thing-to-be-acted-upon" (the concept), second, the object is questioned (search for a way to represent the concept), and third, a desired outcome is reached (representation of the concept), (p. 333).

Adjusting to collaborative learning approaches surfaced within Lily and Connor's AS. This theme proved problematic not only for the dyad but also for the wider class. The first thing Connor had asserted at the beginning of the interview was that he knew how the teaching and learning would be conducted. The project, like others he had previously experienced with New Zealand teachers, included components of group work:

Connor [in English]: When we start you have some rules ... some norms, norms yeah.

Interpreter [ensuring Connor's English was understood]: Because they have already in their mind already decided what they are going to do what you expect.

Connor was forthright in suggesting the project should have incorporated individual work because from his subjective point of view there was nothing to gain from working in pairs through the design process. This tension implied that the direct benefit for him as an individual in succeeding in the activity may become compromised by his dyad partner. However, Lily contradicted Connor. She valued the 
collaborative learning approach, but the way she worked went beyond the boundaries of the dyad:

Interpreter, translating Lily: So, she feels very safe to be together with all the classmates doing design together and when she met some problems her classmates can help her solve it.

The imported collaborative nature of the project aimed to problem solve and share branding concepts within dyadic formations in order to promote design process learning and a studio experience. However, my observations and the data made it clear that when working, the students adopted a system where only half of the class was actively working:

Interpreter, translating Lily: So normally if Lily is cooperating with other friends, usually one is working and the other one is free [laughs]. Not that engaged in the designing.

Connor: [in English] Yeah, but just like she is saying, one work one free, and always have maybe 10-12 persons just work and other person just free, always have those kinds of things.

This middle level tension that relates to the division of labour node in the AS left the same important question that was raised previously. Inside the classroom community and within the division of labour, who is doing what work?

\section{Theme three (3): Navigating imported design practices and processes}

A new theme was evidenced within the AS of this dyad. The rules applied in the classroom were associated with navigating and thus learning imported design practices and processes; these became problematic when Lily evidenced a middle level tension between rules and community. The issue of time constraints related to HNCU rules around timetabling of the project and my imposed no copying rule (which is elaborated on later) was discussed when Lily revealed aspects of her creative process.

She attempted to overcome her difficulties by employing relaxation strategies:

Interpreter, translating Lily: She can only do her design when she is very relaxed, for example, when she was totally free and she wants to play the 
music, or play the video and to let the music or the noise as the background, and then she can, totally relax to think of something, get some idea in her mind, or if she is put into a very stressed, or a very limited time, or very stressed situation just like the class and you ask them to finish within the four periods so she feel very ... [begins to say restrain] . . stressed and she cannot get the idea creatively. So, when she wants to get some idea, normally she would surf on the internet to see the designs by other people, but it's not a plagiarism.

BS: Lily is aware of plagiarism; she knows that is an issue?

Interpreter, translating Lily: Plagiarism, yes, she knows, she knows what it is, she just want to get some inspiration from others' design and then get her own design.

The students used the search engine Baidu to access the internet. But, negotiating the internet is an overwhelming endeavour for students as they navigate their way through thousands of images and concepts. While Lily expressed knowledge of the rules regarding plagiarism, on the second day of teaching in both classes I had discovered a number of examples of copying. Students seated at opposite ends of the classroom were found to be drawing similar teapot logo designs. When questioned about the source of inspiration for their teapot logo designs, students did not defend or attempt to rationalise their work and quickly dismissed their drawings which plainly copied designs from the internet (see Figure 6.4).

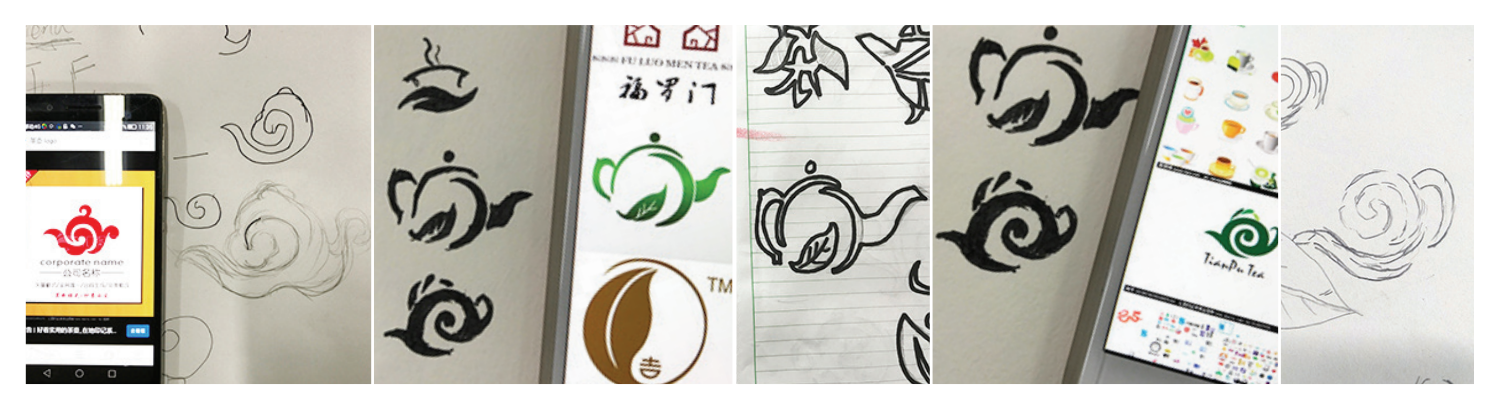

Figure 6.4. Evidence of copying (author images).

A number of student reflections highlighted the practice of turning to the internet for reference:

SR02: I developed a habit of constantly trying and not relying on online materials; 
SR77: I've been in touch with logo design before, but that's not entirely original. It's got some ideas on the web, but the design is entirely my own idea.

An additional middle level conflict was raised when Lily discussed differing learning practices and testing systems. The influence from previous learning affected Lily as she grappled with conflicting New Zealand and Chinese teachers' expectations in the classroom. Her dialogue revealed limited coping strategies when trying to satisfy what she called the testing rules in order to receive good grades:

Interpreter, translating Lily: So Lily mentioned the different requirements, or the different tests by different teachers, just like you the foreign teachers, and the test are different for the students, because Lily thinks that sometimes if they put a lot of effort in doing a design and your teachers think that it's not pleasant it's not good, while when they did something very randomly and not very serious when they did some design, not very serious, but you think that they did a very good job. That's a different test. And, also, she mentioned a Chinese teacher teaching them designing last term [Connor interrupts in English: last year], ok, and the teacher has a lot of requirements from them, and every time they got a design they cannot get approval from the teacher, so they didn't know what to do and how to cope with the teacher's requirements.

The above excerpt reveals complex issues for the students about the value of good work, or what constitutes good work. As New Zealand teachers, the prevailing idea about good work tends to be associated with originality. But for Lily, her confusion about the use of random sketches in the search for originality was palpable. She was confronted by a process she was unfamiliar with. Furthermore, the perception that an unknown or different set of rules would be applied in the evaluation of the branding work was alarming. Conflicting subjective opinions between the students and the New Zealand teachers about the creation of good/original or bad/unoriginal design, combined with completely different opinions reflecting imported and local aesthetic tastes, further exacerbated the issue.

This concludes the findings for this dyad. A summary of key tensions and contradictions that have been discussed is presented (see Figure 6.5). 


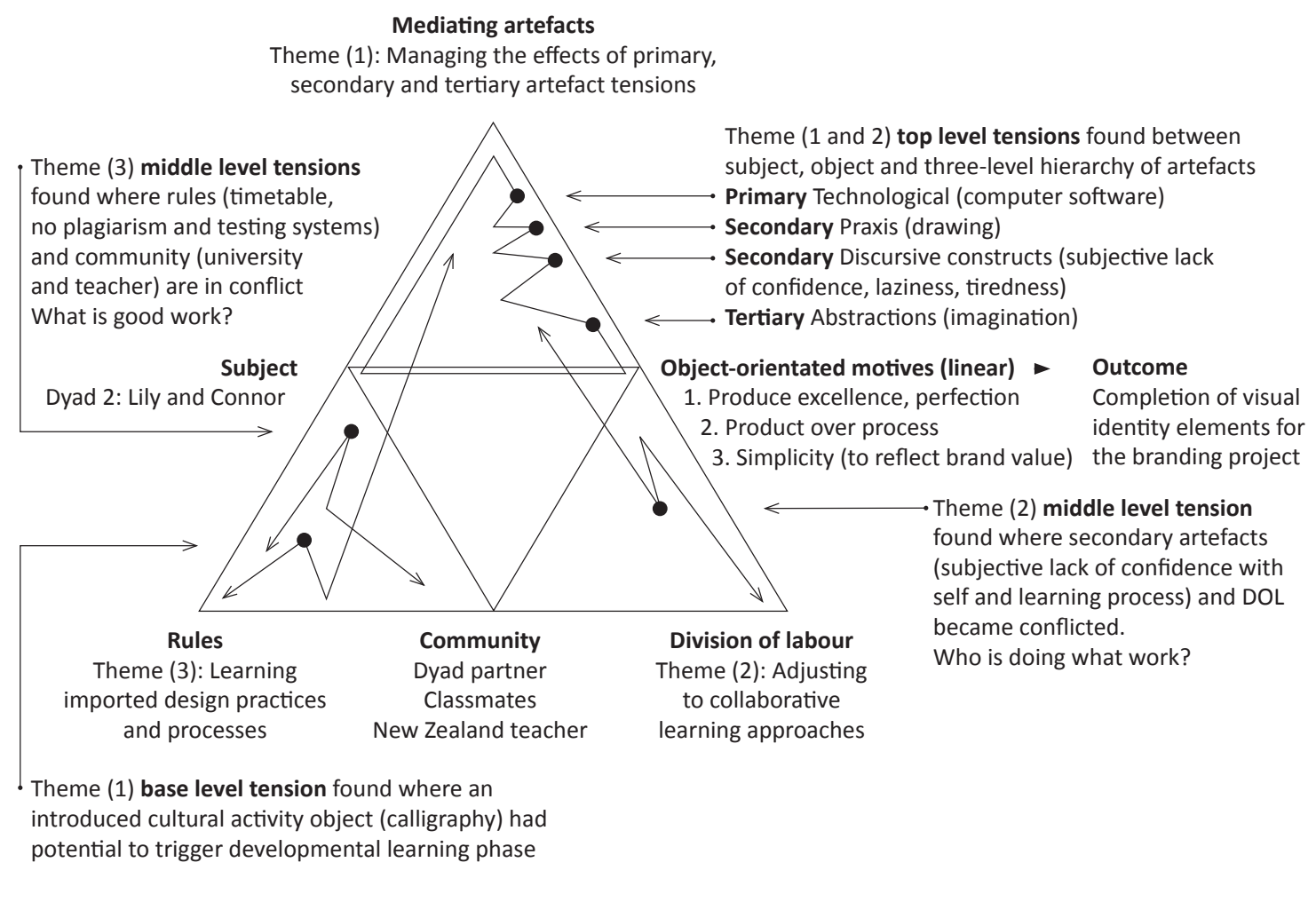

Figure 6.5. Dyad 2: Lily and Connor, themes and tensions.

\section{Dyad 3: Hana and Michael}

Like Lily, Michael was the monitor for class 205. However, unlike Lily, Michael was quieter, and tended to stay close to what appeared to be his three male friends. The group stood out owing to their height as they were taller than their classmates. They also clearly enjoyed listening to music while they worked. Two of the friends would share mobile phone music and a phone cable, with each placing an earphone into one ear. When the four of them did this, they became completely immersed in their individual work. Hana on the other hand spent a lot of time working her way around the class ensuring everyone knew what they were supposed to be doing. I found her actions interesting, because arguably she was aiming for everyone's activities to be aligned to the project outcome. The class on the whole seemed to welcome her interruptions; I never witnessed any dissent.

Within the dyadic activity system of Hana and Michael managing the effects of primary, secondary and tertiary artefact tensions was evidenced again. A fascinating 
discussion began when Hana and Michael were observed on the video footage to be undertaking a shared process:

BS: I'd like you to watch this piece of video and describe what you are drawing, both of you. It's this here, you draw together, can you describe what you are drawing?

Interpreter translating Hana: She created this work first and after that they thought about make a change for the last stroke, so the boy drew this.

BS: Who had the idea, this character what does that mean, can you explain?

Interpreter translating Hana: This tea in Chinese, replaced the line with straight line.

BS: Why did you replace the straight line?

Hana: [In English] This, so we changed this because I think it's not very beautiful so I take that away.

BS: Did that change the meaning?

Hana: [In English] No.

BS: Ok when you were doing this, when you drew the leaf on the side, where did that idea come from?

Interpreter translating Hana: First they took only the single character too ordinary, too common, they think of its not that characteristic of Chinese tea, so they think of making the change, the last stroke, the first idea they want to put the last stroke into branch, branch with leaves here, but a second thought, they think it's better to be a long leaf, a large leaf

BS: And then they went to here?

Interpreter translating Hana: yes, the third.

BS: Is this better, or is this better, how did you decide?

Interpreter translating Hana: [Laughing] So, it's a process, from this, to this, and to this, this, that's the final, that is the final draft.

Hana's description of this process outlined seven actions involving simultaneous deployment of the three-level hierarchy of artefacts. The dyad used pencils (traditional, primary artefact), and hands to sketch (praxis, secondary artefact), the blurred flow of ideas coming from their mind (abstractions, tertiary artefact). Subjectivity was raised, things were 'not very beautiful', or 'too ordinary', or 'too common'. Hana's judgement 
(subjectivity) influenced change and mediated second thoughts, which can be interpreted as transformative. The overall revelation from the above excerpt is that Hana and Michael signalled an object-oriented motive of iterative process learning, which in turn aligned with my iterative process teaching motive. Therefore, the outcome of their learning at that particular moment was not focussed on a linear process; rather, the iterative process became the outcome. Their object-oriented motive in this instance was smaller than Engeström's interpretation as it points to Leontyev's $(1981,2009)$ interpretation of object. The object of the activity gave their process a determined direction and so the dyad was satisfied at each endpoint (sketch) or outcome of the process. Hana chose to use this concept later in the test (evaluate) design thinking phase for one of her completed outcomes (see Figure 6.6). This figure depicts the dyadic process as was described in the interview above, and Hana's individual computer development in the design thinking test (evaluate) phase which continued her iterative process.

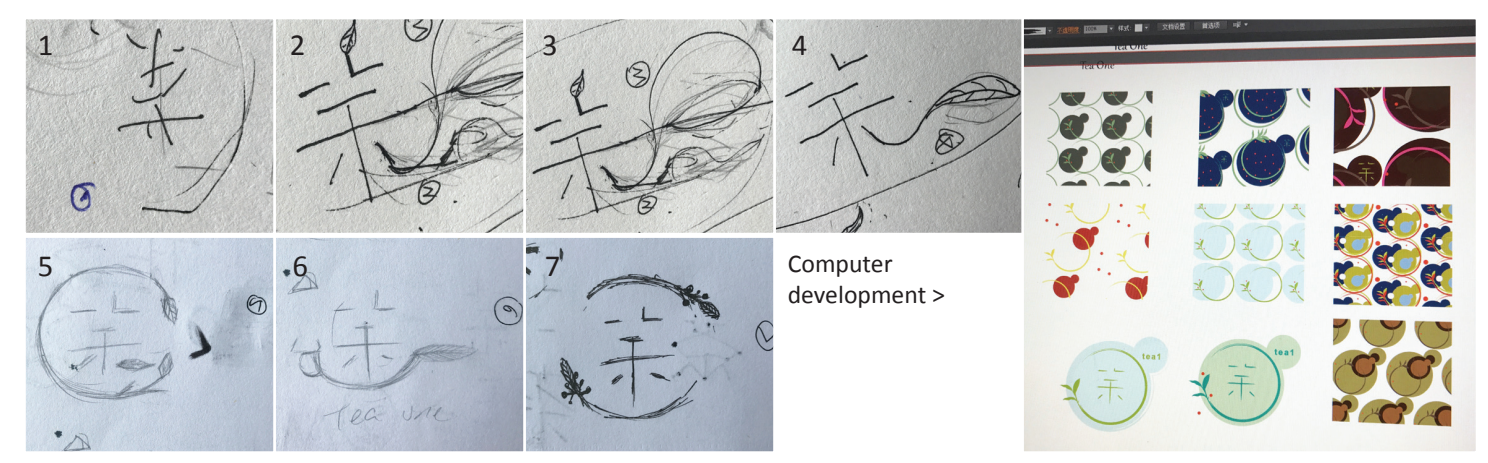

Figure 6.6. The dyadic process and Hana's subsequent computer development of the concept.

Michael illustrated how creative design processes can emerge from an incubation stage of creativity with an a-ha moment (illumination or design epiphany) which was evidenced on the video. The result of his epiphany was mediated by an emotional secondary artefact, happiness:

BS: So then what happened because it's quite interesting, at this point, see when you did that, and you say something, you go a-ha . . can you describe that feeling to me? What went through your mind?

Interpreter translating Michael: Because at that time he was very satisfied with the design. Because at that time he was how do you say, he found that 
he got he has got what he wanted to express, so he was very excited, and happy.

A reference to simplicity (see below), also raised by Connor in the previous dyad discussion, may have revealed an object-oriented motive in the tradition of Engeström, by Michael. Engeström's interpretation of object was defined by community interaction and culturally mediated human activity or activity systems (Engeström \& Miettinen, 1999). To explain, Michael became motivated to keep persevering with his work when he realised simplicity could lead to something very special. This was interpreted as holy high, a particular Chinese turn of phrase that I learnt about during the interview. Furthermore, Chinese values came into play that can be linked to both a tertiary artefact, and an emotional secondary artefact:

BS: How did you manage the overall process of this course?

Interpreter translating Hana: From the heart, heart and soul

BS: Michael?

Interpreter translating Michael: Imagination.

Imagination. Can you elaborate more?

Interpreter translating Michael: To use his imagination to combine all the elements together, to keep trying. After a lot of experiments he sensed that the logo, the simpler the better.

BS: Can he tell me why he thinks simple is better?

Interpreter translating Michael: It looks comfortable, and very convenient to get the meaning [Holy high heard in the dialogue].

BS: What is holy high?

Interpreter: That's colloquial in Chinese, it means something that is very sacred, is very noble.

Hana's expression of heart and soul is an emotional reflection related to love which in turn reflects Michaels previous assertion of happiness driving a design process. However, Hana expressed reservations which involved bravery, and signals considerable effort and risk taking: 
BS: How would you begin the project again?

Interpreter translating Hana: So first she would like say, if she go back to the very beginning, she would not stick to the original ideas, she would bravely try some more experiments, because the more experiments she does, the more she can get, the more ideas she can get for the experiments.

Similarly, a number of student reflections echoed Hana's sentiments:

SR03: We must be brave to try, don't give up, always design good;

SR68: At first I decided that a sign didn't want to change. But then I tried a variety of other designs, and I got a lot of surprises. I realised that I should be brave to try many ways when designing ... I'm going to try and be brave when I'm designing, and I'm going to look at other designs, hope I can make better designs;

SR75: I dare not brave innovation ... I can't be lazy. I need to work hard.

Although the previous two dyads evidenced very clear product over process tensions, Hana and Michael contradict these findings. Michael said he tried lots of experiments, and the pair were observed undertaking an iterative process. The above excerpt reveals Hana's awareness of process but her lack of bravery restricted, to a certain extent, her willingness to actually engage with a wider experimental, and therefore iterative process for longer periods of time. As the interview progressed, Hana started to talk about the role a computer plays in design. She said she thought that the computer was very good for design. This intriguing point was followed up by the next except:

Interpreter translating Hana: She holds the opinion if she does the drawing on the paper first and then put it into the computer, then the design is very good, its better, the design is better.

BS: How was working this way good?

Interpreter translating Hana: Yes, the drawing first, and the computer second, this way is better than the way we do on the computer.

BS: Is this an unusual way for you to work?

Hana: [in English] Yes, I think it is amazing. 
This alluded to differences in expectations and ways of doing between New Zealand and Chinese teachers, and it was unclear how idea development through drawing is used in other learning situations.

This dyad extended their learning beyond the bounds of their dyad partnership, and therefore the theme adjusting to collaborative learning approaches was evidenced again. Hana was observed in the video footage as being instrumental in involving all four participants in the early stages of the process. Conceivably, the motives of all four participants may have been reshaped by increased activity through the interaction that occurred between the two activity systems:

BS: How was it helpful to work with everyone?

Interpreter, translating Hana: When two work together they only have two ideas, but if they can work with other group they can get more ideas.

BS: How did that work for you Michael as you were sitting on the end? Interpreter, translating Michael: If they can work with four people, if four people work together they can get much more ideas, and he can choose some of the ideas that suit him from a lot of ideas, while when they only two of them work together, it equals one plus one, so he can have more choices, when he works with the larger group.

It would appear from this excerpt that Michael's motive was based on affordances of increased ideation when the two activity systems overlapped. Overlapping activity systems signals third generation activity theory, but I did not pursue this in a diagrammatic form as the analysis of the fourth dyad was yet to come. Furthermore, at this point in the research I was not clear if Hana and Michael's experiences and assertions were an anomaly or if they would prove consistent with other dyads. The pair avoided discussions of negative experiences; they preferred to present a positive attitude and openness towards the class teaching and learning. This suggests they had experienced a number of transformative moments owing to their willingness to engage with the uncertainty of solving design problems. A diagram is presented which depicts key findings from the third dyadic interaction (see Figure 6.7). Of note from this analysis is that within this particular dyadic AS, two different object-oriented motives were evidenced and both accepted iterative practices in design learning. 


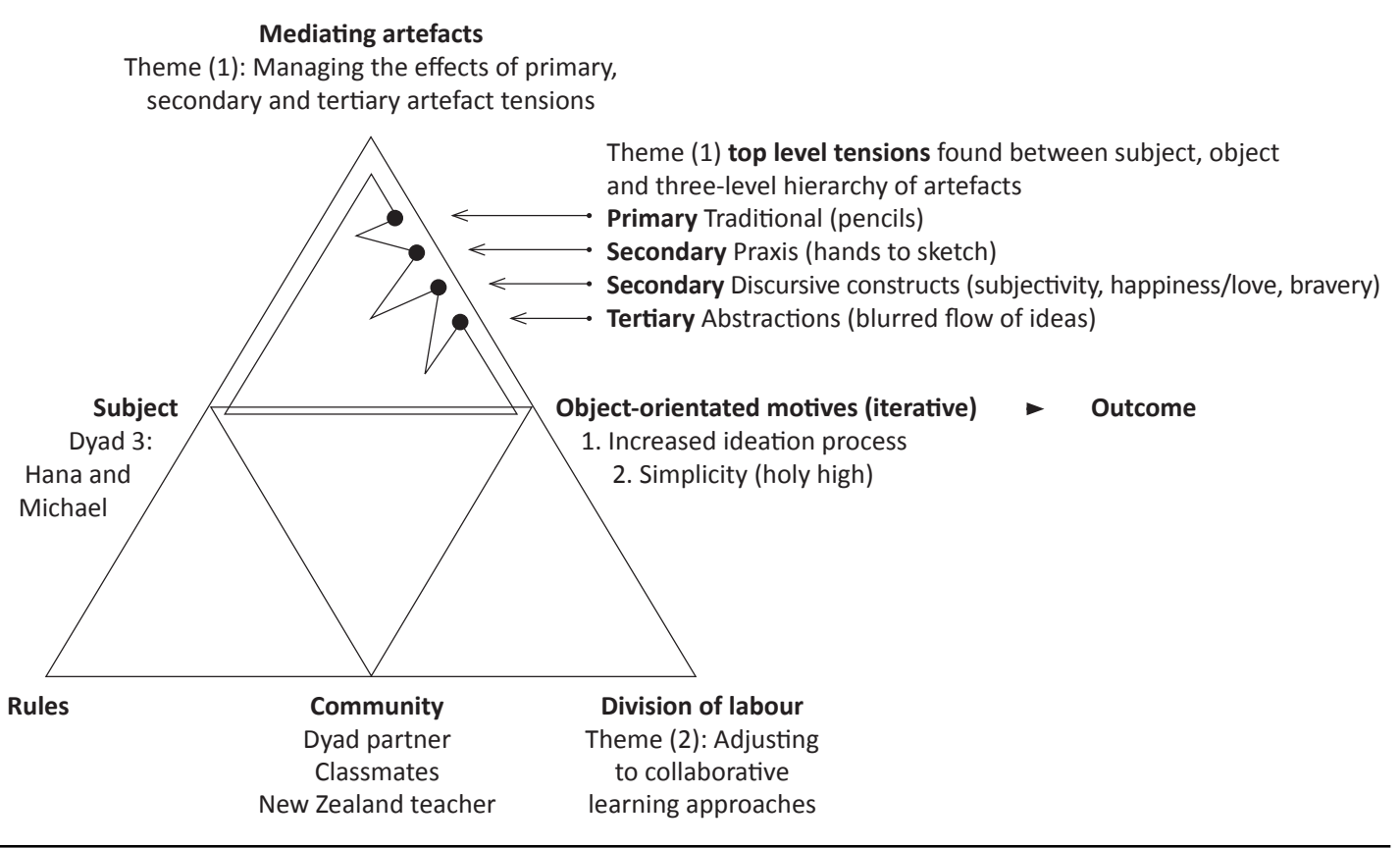

Figure 6.7. Dyad 3: Hana and Michael, themes and tensions.

\section{Dyad 4: Ricky and Simon}

Ricky and Simon were two of Michael's friends who I talked about previously. Of the pair, Simon was the more industrious, whereas Ricky seemed distracted by female attention. At the beginning of the project Ricky was sitting with his friends and a girl was seated beside him. However, in the middle of the project there must have been an altercation, and the girl, visibly upset, moved to the other end of the classroom. Ricky seemed unconcerned, and his interactions with his friends and dyad partner continued with their practice of listening to music while they worked.

A similar pattern of themes and tensions was revealed in this analysis when Simon described his creative ideas as originating from personal life experience. Managing the effects of primary, secondary and tertiary artefact tensions started to reveal how his cultural learning background might have influenced his actions and motives. Simon talked about sketching being an important component of his idea generation:

Interpreter, translating Simon: He says some of the ideas are coming from the real life, past experiences, but some of them coming from the practice, the real practice when he's drawing, when he's sketching, so he can get some ideas. 
BS: Can you explain this in more detail?

Interpreter, translating Simon: After he draw some sketches, he examines the sketch for a little while and can get something relevant to the sketch, get some idea.

Essentially, Simon is talking about a creative practice whereby the materiality of the sketch provided inspiration. However, I observed that he did not spend a lot of time doing this; rather, his reliance on the computer to develop a single idea led to an interplay between love, an emotional secondary artefact, and the computer, a primary artefact. This connection and Simon's dialogue revealed object-oriented motives of perfection and product over process:

Interpreter, translating Simon: At the very beginning, he thought that his drawings are a mess, chaos, so he didn't have high expectations of the drawings, but after several experiments he found some feeling towards the design and after using the computer he's sensed the effect is becoming better and better.

BS: What is the effect?

Interpreter translating Simon: The visual effects, the visual, how do you say. He sensed that after the logo is designed in the computer [it] is becoming better and better, good design and he began to love it.

BS: If you were to repeat this project, how might you organise things differently with your partner?

Interpreter translating Simon: If start again from the beginning, he would not draw the paintings so complex. There is no need to make the drawings very perfect.

BS: Why is there no need to make the drawings perfect?

Interpreter translating Simon: Because everything on the computer, it becomes better.

Neither Simon nor Ricky were happy with their final outcomes but were unwilling to change or repeat the drawing exercises when questioned. It seemed that in dealing with their perceptions of unappealing aesthetics, further adaptation was too difficult.

Whether this was laziness, or that a design block was encountered, or that there was a refusal to engage with an iterative process, it was unclear because there seemed to be a lack of questioning or evaluation in the learning actions. As a result, it can be argued 
that there were tensions between my object-oriented motives which involved teaching new design practices and processes and the dyad object-oriented motives which were directed towards straightforward or linear pathways of learning and outcomes focussed on goals of completion:

BS: Did he think about going back and re-doing his drawings?

Interpreter translating Simon: No, because it's too ugly, it's hard to make it more beautiful.

Ricky struggled with generating new conceptual ideas and his creativity, blaming his inability to make a start or move forward on his lack of experience with the product:

BS: What holds you back in your learning?

Interpreter translating Ricky: Because sometimes if the product is totally new to them and they don't have so much experience with the product, they cannot get any idea from the memory, so it's very hard for them, for him to think about some ideas, relevant with the product.

A number of student reflections were found, which supported Ricky's issues with the difficulties associated with making a start on the creative process:

SR08: The difficulty lies in creativity. The first thing to do in design is to have ideas ... my knowledge has defects and deficiencies;

SR32: I am not very deep in graphic design and lack of imagination;

SR47: For about a week I had no idea, I am struggling;

SR54: The difficulty lies in the idea. I always think my idea is not good enough. I'm afraid that my own is not good enough;

SR92: Continuous design makes it more difficult for me, because I find myself at a loss ... I have no feasible ideas in my mind.

Moving to the next theme, adjusting to collaborative learning approaches, Ricky preferred the social exchange of shared group work over an individual learning experience:

Interpreter, translating Ricky: So if he work alone, he feels lonely and then if they work together, they can get more creations, better creations. 
My observational field notes record that neither Ricky nor Simon sought help from the wider classroom, although they were involved with Hana and Michael (dyad 3) as discussed above. On further questioning about their friendship, it was revealed that the two participants were living in the student dormitory on the campus. My questions around their accommodation situation led naturally to a piece of video footage where Ricky was observed to be working alone after Simon fell asleep with his head on the desk. Once more the question, who is doing the work? was raised. The next excerpt highlights the challenges not only that this dyad faced, but all students who live in the dormitories face when their design work becomes compromised by a middle level tension in which tiredness, a secondary artefact, conflicts with the DOL:

BS: I don't want to embarrass [Simon] but he fell asleep on the video footage. He was very tired. Can he tell me why was he so tired?

Interpreter, translating Simon: They stayed up too late.

BS: You were tired too [Ricky]?

Interpreter, translating Ricky: They two stayed up very late.

BS: Are you room-mates?

Interpreter, translating Simon and Ricky: Yes and no.

BS: But do you spend time together?

Interpreter, translating Simon and Ricky: Yes.

BS: What where you doing staying up late?

Interpreter, translating Simon and Ricky: Because the other students did not go to bed, so cannot sleep.

Further questioning did not reveal how Ricky felt about conducting the design activity alone, nor did the video evidence any ill feeling about this when Simon woke up. The bell woke Simon up, and the pair left their seats for a break.

This concludes the analysis of this dyad; a diagram is presented which depicts key findings from the fourth dyadic interaction (see Figure 6.8). 


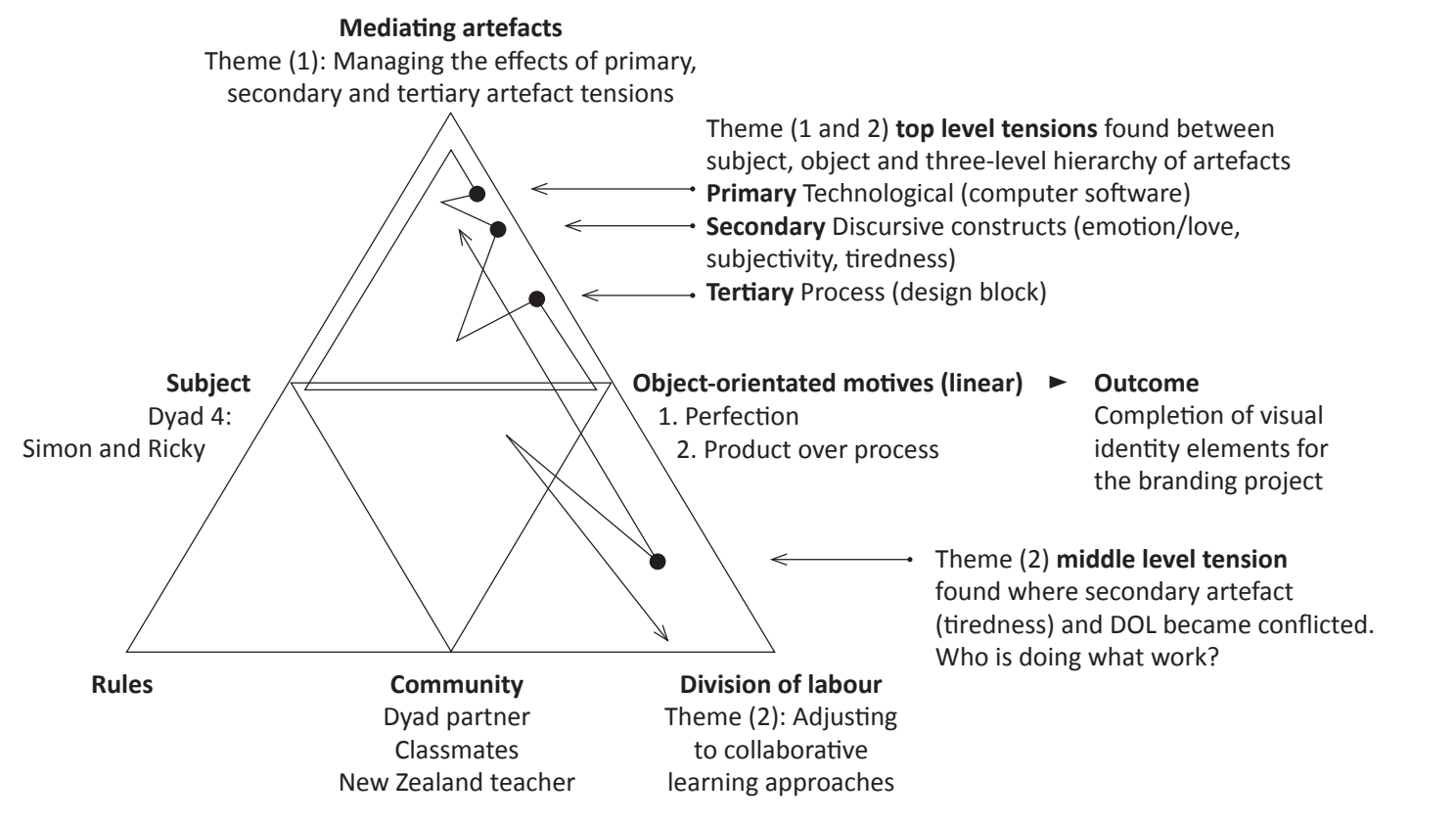

Figure 6.8. Dyad 4: Simon and Ricky, themes and tensions.

The activity system top level tensions from this dyad did hint at how their cultural learning background might have influenced individual actions and motivation. The way Simon talked about the value of sketching, in conjunction with superior drawing skills evidenced by most in the class, gave me some cause to wonder about previous learning experience. Yet, despite these apparent skills, the dyad resisted any changes to their drawings once they perceived they were perfect. Their process was strictly linear. This in conjunction with an emotional connection and perceived mastery of the computer to improve the work further, pointed to object-oriented motives of product over process, and outcomes of completion. 


\subsection{Discussion and conclusion}

Key themes and findings from the four dyadic interactions and student relections are combined and presented for this dicussion and conclusion (see Figure 6.9).

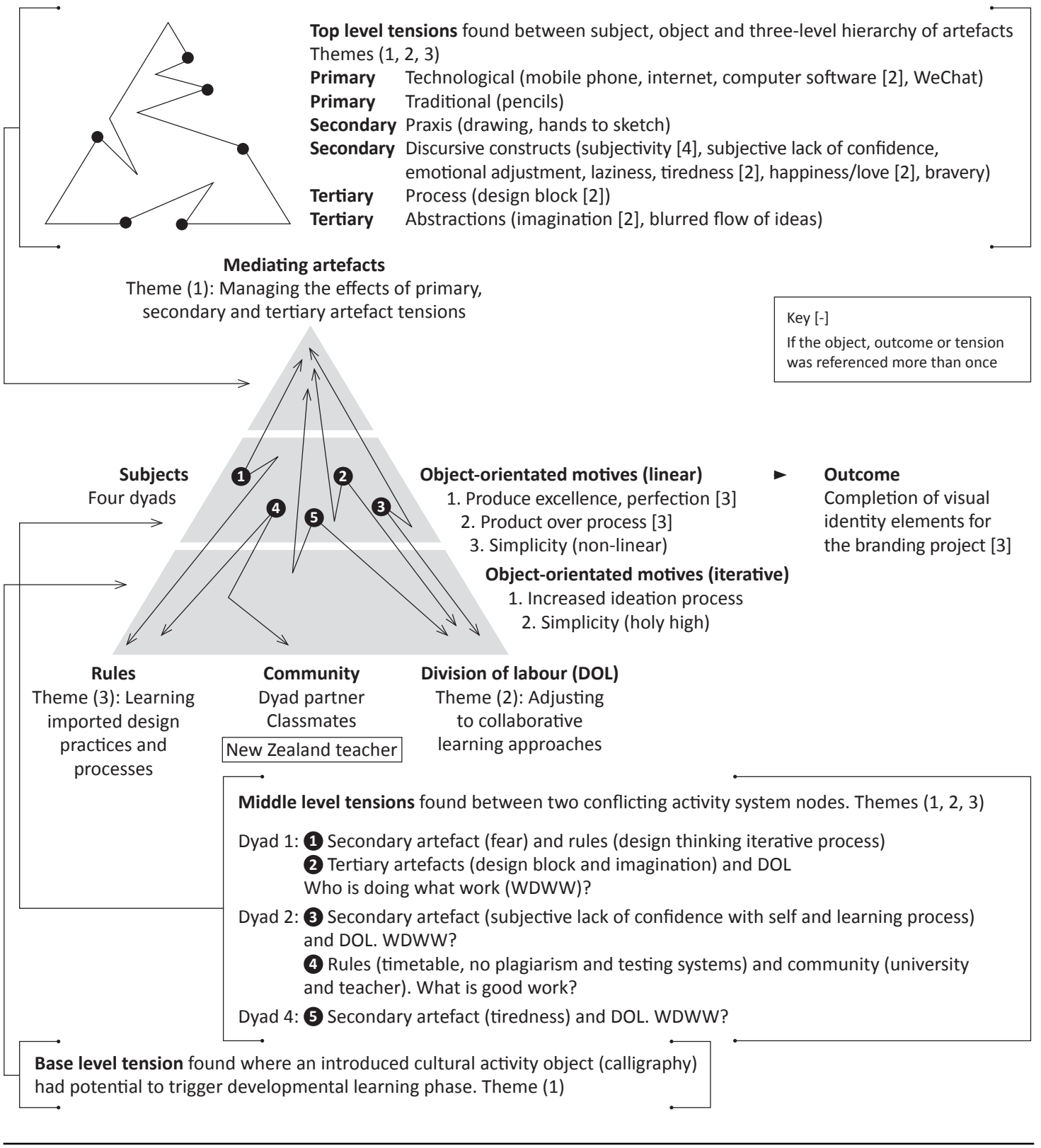

Figure 6.9. Branding project: Key themes and findings from combined dyadic interactions, observations and reflections.

Within the branding case study, three key themes were found from combined dyadic activity systems, and tensions and contradictions were linked to these themes in the figure above: 
1. Mediating artefacts: Managing the effects of primary, secondary and tertiary artefact tensions;

2. Division of labour: Adjusting to collaborative learning approaches;

3. Rules: Navigating imported design practices and processes.

All themes were evident throughout the entire activity system top, middle, and base levels, according to Leont'ev's characterisation of a three-level model of activity theory, Foot's (2014) "levels of contradictions and corresponding learning actions" (p. 342), and Engeström's ideas about object-oriented motives expanding and including collective social and cultural contexts to account for different traditions and perspectives (Engeström, 2001; Engeström \& Glăveanu, 2012).

There was a multiplication of some issues found at the top level of the combined activity system in the managing effects of primary, secondray and tertairy artefact tensions theme. Computer software related tensions were the most problematic primary artefact issues and secondary discursively constructed problems revealed four instances of subjectivity and two instances of tiredness, happiness/love respectively. The two instances of data which referenced design block and imagination were attributed to tertiary artefact tensions. At this top level, three of the dyads were found to be driven by object-oriented motives of product over process and perfection. This key finding means that there may have been a perception by the students that if they made the work perfect, the benefit or result of the completion may result in a higher grade. Furthermore, I argue that object-oriented motives of product over process and perfection are linked, as potentially they derive from historic trajectories of learning where these ways of doing may have been taught. Motives of product over process/perfection and goals of completion were found to be inextricably tied to a linear pathway. An example of this was evidenced by Amy who was focussed on the end of the project with her first design concept, and she would have preferred to continue working in a one-way, continuous direction. She became upset and was fearful when other options were suggested by the teacher. Furthermore, on reflection at the end of the project both Amy and John felt convinced that it was better to set the direction and focus on a goal of completion rather than use an iterative process. However, there were a number of student reflections that contradicted the dyads' 
opinions; many were intrigued and embraced an iterative process but in doing so a great deal of encouragement was required. Typically, students tended to wait for the teacher to approve their work before moving on to the next stage, which indicates a lack of individual readiness to initiate further action in the design process. Objectoriented motives of simplicity were mentioned twice. Connor talked about it in relation to an imaginary logo that he hoped to design which was as perfect or as multidimensional as the apple logo. The second time it was mentioned was in association with the Chinese expression 'holy high' and a link to a participant object-oriented motive in the tradition of Engeström (2001) was established. Michael's dialogue about 'holy high' evidenced an interpretation of object which privileged social, cultural and community elements which in turn drew on other activity systems (Engeström \& Miettinen, 1999).

Middle level activity system tensions where two AS nodes came into conflict led to two questions: first, who is doing what work? and second, what is good work? (Engeström, 1993; Foot, 2014). Issues arising from artefacts and division of labour were implicated the most in who is doing what work; for example, when Simon fell asleep through tiredness Ricky continued the work. What constitutes good work was discussed in relation to the prevailing idea New Zealand teachers tend to hold which associate good with originality. Differences in subjective opinions arose between the students and the teachers about this which led to a certain amount of disorientation and confusion for the students who were confronted by an iterative design thinking process they were unfamiliar with. They worried about a different set of rules being applied in the evaluation of the branding work which signalled tensions associated with learning imported practices and processes. However, there was a moment when the motives of one dyad - Hana and Michael - became linked to the second theme, adjusting to collaborative approaches. They drew benefit from each other's actions in the idea generation process to reveal a motive of increased ideation which in turn points to functions of "construction, generation, and creation of new" (Tikhomirov, 1999, pp. 349-350). This finding means that there was one instance of dyadic activity that aligned to my motives of teaching iterative process learning for increased ideation. The outcome of their learning at that particular moment was not focussed on a linear process; rather, the iterative process became the outcome which revealed that their 
object-oriented motive was smaller than Engeström's (2001) interpretation. The object of the activity gave their process a determined direction and so the dyad was satisfied with the outcome of this non-completed aspect of the process (Leontyev, 1981).

One base level activity system tension was revealed when Connor introduced calligraphy to his practice to challenge the rule which stipulated thumbnail sketches be used for idea generation. This base level tension proved the second example of an object-oriented motive as it was interpreted by Engeström (2001). Connor's attempts to consolidate a new practice through the use of calligraphy drew on past and present teaching and learning experiences. Furthermore, Foot's (2014) three facets of the problem space were evidenced: first, the concept was acted upon; second, the object searched for a way to represent the concept; and third, a desired representation of the concept may have been reached. Ultimately, Connor did not follow this process through, but the potential was raised for a developmental learning phase to be evidenced.

This chapter sought to understand and ouline the processes of dyads working on a branding project using design thinking in VCD learning at HNCU. Key to the analysis was the discovery and adaptation of the three-level hierarchy of artefacts (Cole, 1999; Wartofsky, 1979). Prior to this, it had been tremendously difficult to understand or even conceptualise how a tangible artefact such as a pencil or a computer could be in any way related to an intangible thought process or personal feeling or emotion. Yet, in my experience, if a student was unhappy or frustrated, this would affect how they engaged and acted upon a learning object. In other words, their state of mind could mediate their outcome. Moreover, the notion that tertiary artefacts could involve possible or imagined worlds opened up many avenues for this analysis. Therefore, in speculating about future constructs and linkages of findings from this case study to the next which follows in the next chapter, I believed that a similar pattern of dyad motives, outcomes and tensions would be revealed. 


\section{Chapter 7. Miao case study}

Using a cultural project to teach creative design processes aimed to provide students with a theme or topic that was familiar. The project drew on cultural motifs from local Miao indigenous tribal stories to create a character illustration design for a deck of playing cards (Saris, 2018). The focus of learning was to nurture a fluid iterative process within the design thinking ideation and prototype phases. The focus of the analysis was to uncover tensions and contractions as learning occurs when VCD students are exposed to a new pedagogy. Similar to the branding project, four separate dyadic activity systems were analysed for this case study. Insights and emergent themes are presented from eight individual students situated in the four dyads as they participated in a design process activity in their second year of study. The project was taught at Hunan City University (HNCU) in China for three weeks, from $21^{\text {st }}$ May to $8^{\text {th }}$ June, 2018, with the research interviews conducted in the last week of this period. As was outlined in the previous case study, I was timetabled to teach the same two classes as I had taught in the first case study, identified by the numbers 203 and 205. Participants for dyads one and two were recruited from class number 203, and, participants for dyads three and four were recruited from class number 205 (see Figure 7.1) which details pseudonyms used in place of actual student names.

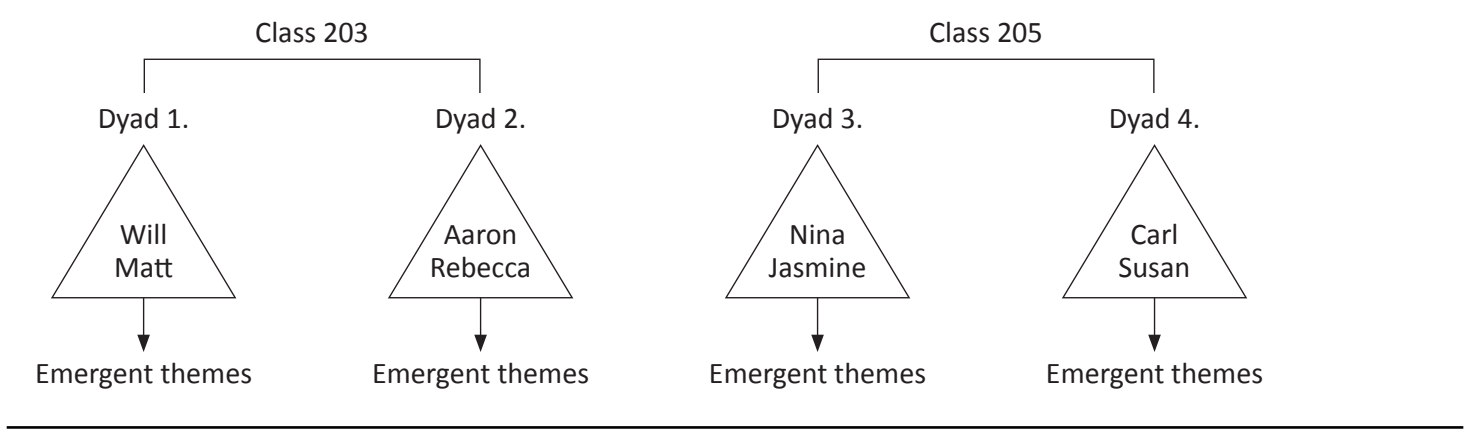

Figure 7.1. Case study two: Miao playing card project. Four dyadic activity systems.

The three-level hierarchy of artefacts was used to analyse insights and form themes from the four separate dyads. To recap briefly, primary artefacts are those traditional or technological tools such as a pencil or computer that are used in basic operations and VCD production. Secondary artefacts include the hands-on praxis, or discursive constructs such as subjective and emotional factors which may influence the 
deployment of primary artefacts. Tertiary artefacts use abstract and imaginary processes that occur in the mind. As outlined in the previous chapter, the analysis approach drew from Leont'ev's theorisation of a three-level model of activity theory as detailed by Engeström and Miettinen (1999), Foot's (2014) descriptions of the characteristics of tensions and contradictions occurring at the top, middle and base levels of an activity system, and Engeström's (1993) assertions that the wider contexts of an activity system be included. Furthermore, the distinctions made between Leontiev and Engeström in regard to object were taken into account after data from the branding project revealed differing object-oriented motives were in play (Kaptelinin, 2005). Additional data from 100 student reflections that were collected from all students who participated in the project are included in the analysis that follows.

To conclude this chapter, the four dyadic activity systems are combined into one overall AS for a discussion. Students were found to be disturbed by an iterative process, and the role of previous learning was implicated which led to a change of research design. I now begin with the analysis of the first dyad activity system, Will and Matt.

\subsection{Analysis}

\section{Dyad 1: Will and Matt}

The friendship of Will and Matt was obvious; they were a humorous pair, often cracking jokes, or showing each other online memes on their phones. They tended to keep to themselves in the classroom, choosing to sit off to the side of the room away from the row of 'cool' boys at the back. In the video footage Matt evidenced very early on issues surrounding the first theme, managing the effects of primary, secondary and tertiary artefact tensions that had surfaced in the previous case study.

Matt was seen to be rapidly moving in and out of Illustrator, Photoshop and image searches on the internet, which appeared to be unrelated to his design work. An objective of the project was to use simple shapes to design a character illustration which considered ways to use form in an original and contemporary manner. Matt's 
activities on the video raised questions for me, because the students had been instructed to use Illustrator to construct vector (line) images, rather than using Photoshop which generates pixels. One function of Illustrator enables a technique called Image Trace. This function automatically converts a photographic image (pixels) to vector imagery (line). However, the technique limits the opportunity to further manipulate the traced image due to the high number of vector paths that are produced. Moreover, the student does not enter the design process as they are not creating an original design in a considered way. However, by beginning with simple shapes and using the Pathfinder and Shape Builder tools in Illustrator, these issues are eliminated as the linear paths are simple and easily manipulated to create smooth, curvilinear forms (see Figure 7.2).

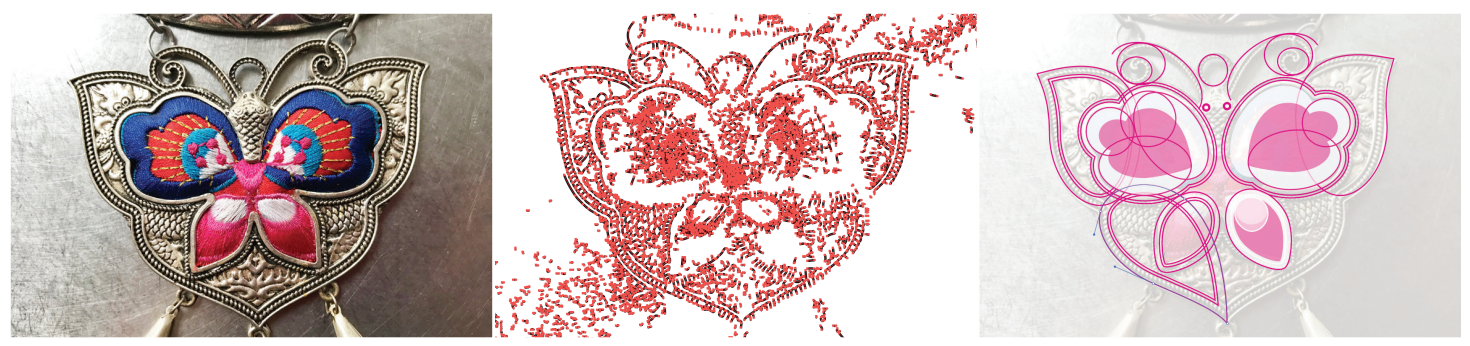

Figure 7.2. Illustrator image trace technique: 1. Original photograph (author image), 2. Image trace function applied, and high number of vector paths are generated, 3. Example of constructing a form using simple shapes.

I observed a great number of the students, including Matt, beginning their design process by using the Image Trace technique despite explicit instructions conveyed through the interpreter not to use this approach. The video showed Matt using Image Trace, and I could not understand why he kept deleting what he was doing:

BS: After nine minutes and 30 seconds he quit illustrator, and it appeared he was working on something else. He had all these images of houses, and he kept going in and out, in and out of these, I would really like to know what he was doing.

Interpreter, translating Matt: So, at that time, because, he's totally lost about how to design the face of the dog, because you know, for the dog sculpture there are no details on the face, he's thinking how to add some details to the face of the dog. So, at the time he is very depressed that he could not find a best way to improve the image, so he went to some other parts, to take a rest for several minutes before coming to the design again.

BS: He did that for a good 40 minutes, why was it a big problem? 
Interpreter, translating Matt: So, it's also the difficulty of the idea, yeah, because at that time, he is really frustrated and cannot find a way to go through, so he went to the other parts to other things to let his mind free.

At the time of the interview I was perplexed that a student would persist for so long with the technique but was assured that Matt's activities had nothing to do with any other class or homework that he might have been doing in class time. Thus, Matt's interpreted discussion demonstrates the tensions or design block he encountered during his design process whilst trying to activate illumination and deploy the software:

Interpreter, translating Matt: He only has an abstract idea of the image in his head, but it's very difficult to present it.

Two student reflections (SR) add support to Matt's difficulties. The first is explicit in recognising that the effects of primary, secondary and tertiary artefact tensions require management if the student is to be successful in a future workplace. The second reflection is less specific but recognises the notion of personal subjectivity and self-criticism in the workplace being something that would have to be overcome in the future:

SR74: I didn't like the theme of buffalo very much at the beginning, but later I thought that a professional designer could do anything well;

SR78: Of course, sometimes when I am designing, I'm going to be emotional, I'm lazy, I'm not serious. I know that if I want to be a qualified designer these shortcomings are not allowed, so I will push them away from me a little bit.

In the previous case study, the theme, adjusting to collaborative learning approaches had emerged within the division of labour. After discussions with Will and Matt the same theme arose, evidencing the positive way in which the students work with each other, and suggesting that adjustment to this way of working may have come quite naturally to the them, yet there was a reluctance to offer any form of critique. When questioned about the positive aspects of shared learning practices, this dyad was more than willing to discuss benefits (strengths/advantages) of the partnership. The socially enjoyable aspects of collaboration are evidenced in Will's dialogue. Matt's singing 
promoted happiness, and his help with the technical aspects of the course was perceived to be beneficial:

Interpreter, translating Will: Matt gives him a sense of happiness because when they work together [Will interjects], when they are working on the design he doesn't feel the how do you say, it's not boring, it's not boring to work, and also, the cooperation is very effective with two people with two people working together, and so when it also, he enjoyed the whole process of working together, and Matt can teach him a lot, yeah in addition, Matt's singing is good.

Following this exchange, it proved difficult to elicit any data that were negative. Both Will and Matt were very reluctant to offer any critique about each other's work, which was reflective of Hana's and Michael's discussions during the branding project. Hana and Michael had similarly avoided any exchange of negative experiences. It appeared that peer critique was uncommon and that students were uncomfortable with this practice.

In order to create a consistent colour palette for the deck of playing cards, the educators provided 24 colour swatches, which were selected through colour matching from reference photos of Miao cultural artefacts. The colours were made up of cyan, magenta, yellow and black print specifications, and ranged through primary (red, yellow, blue) and secondary (orange, green, purple) tints and shades. Essentially, the directive or rule to utilise any number of the 24 specified colours was found to produce conflict with subjectivity, a secondary artefact, and imagination, a tertiary artefact. This middle level tension where two nodes of the activity system, rules and artefacts, came into conflict proved quite problematic. It seems there was a tension between the rule and the capacity or ability of the dyad to confidently settle on a colour scheme for their illustrations. The first excerpt evidences Matt's difficulty with his mind in imagining or envisaging a colour scheme for his design, resulting in frustration with his progress, and a strategic attempt to seek other student ideas to resolve his issues:

Interpreter, translating Matt: Because other students are doing faster than he, his mind is confined to ideas, so it's very slow in designing all the image. So, when the other students, almost finished their design, he looked at their designs to get some hint from their use of colour, so he finds that 
their use of colour shows a kind of harmony, so, he borrowed the idea from the colour to make the colour scheme to his design.

The issue of colour selection for the illustrations was widely discussed in the student reflections. Of the 100 reflections, 38 (or almost 40\%) students discussed their problems. For example:

SR06: For me, colour and pattern are my blind spots. I hardly have the right choice for these two items;

SR19: When I started to colour, I had problems again. I was not sensitive to colour, and my own colour was very ugly;

SR20: The bad colour combination made my work dim;

SR33: Some controls on the colours that have caused me to encounter great difficulties;

SR51: Colour matching is very difficult for me. I don't feel very good about colour;

SR56: I think what annoyed me most in this lesson is the handling of the whole colour;

SR61: I was in trouble to complete the colour problem of the design;

SR81: How to match the colour has become my distress.

Recorded in my field notes was a mention of colour being problematic for the students. The students seemed to be drawn to drab colour palettes and tended to choose the similar toned orange and bottle green, despite the opportunity to select from any number of contrasting contemporary colours. This may have been because these two colours were situated in close proximity to each other on the colour palette. Or, the more likely scenario was that often the students tried to use colours that represented their character in a realistic way. But the choices were surprising given many of the students were wearing vividly coloured clothes and the surrounding environment of Yiyang was predominantly colourful.

From this dyadic interaction, a diagram is presented which depicts emergent themes from the findings (see Figure 7.3). 


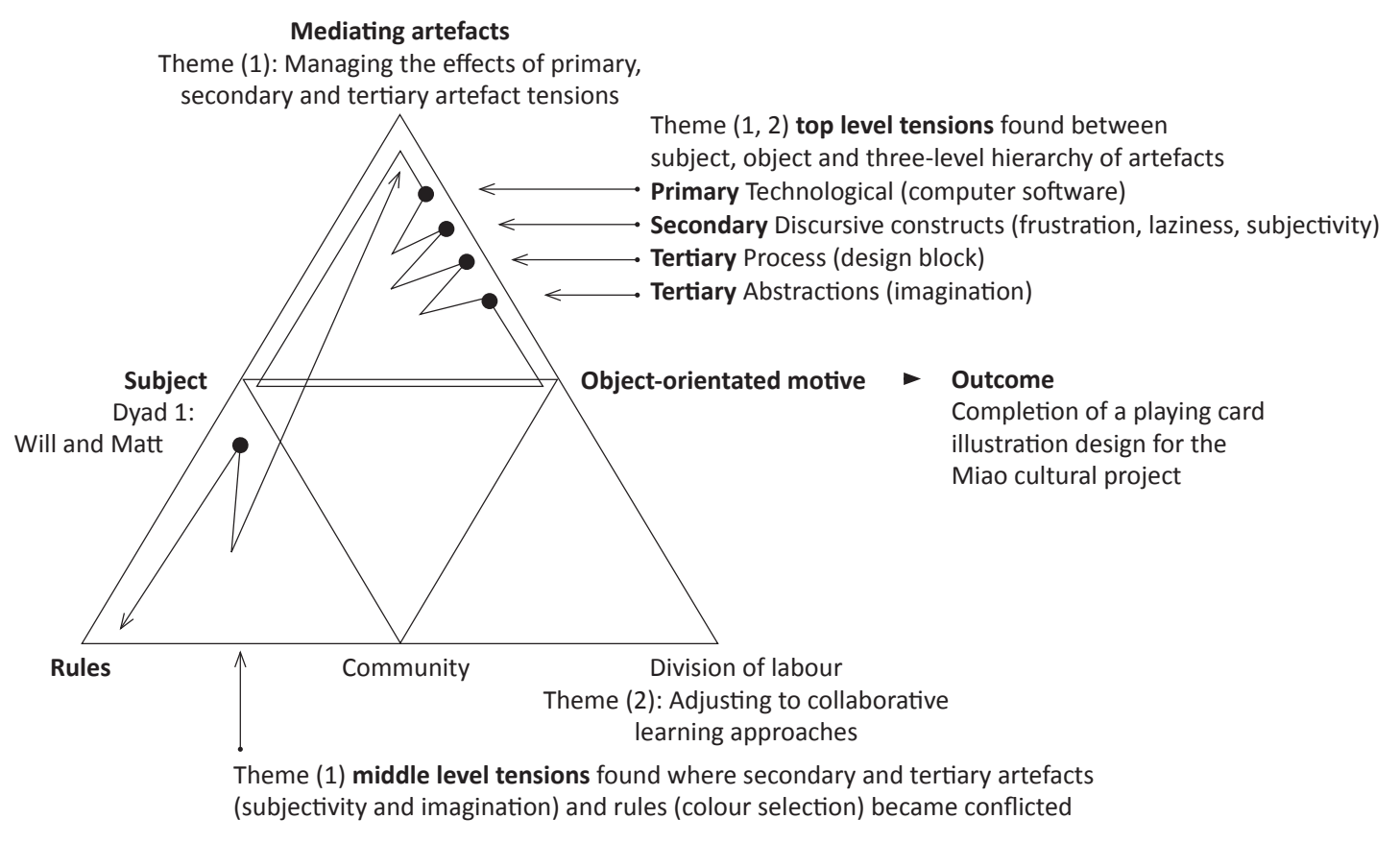

Figure 7.3. Dyad 1: Will and Matt, themes and tensions.

\section{Dyad 2: Aaron and Rebecca}

Of all the student participants, Aaron was the one who I felt I grew to know the best. Like Connor in the branding project, Aaron's grasp of English was excellent and so it was easy to converse with him during the interviews as well as in the classroom. $\mathrm{He}$ was incredibly hardworking, and more often than not positioned himself at the front of the class to ensure he was on hand when I asked questions during class time. Rebecca seemed quite the opposite to Aaron. She was shy and spoke very little English, so it was more difficult to interact with her.

The theme managing the effects of primary, secondary and tertiary artefact tensions was raised by Aaron, who had similar issues to Matt when he discussed two main problems associated with the idea of a generation (drawing) phase. The first issue he discussed was when he tried to decide on the general direction of the design, and the second involved development of his ideas:

Interpreter, translating Aaron: So when he started to design the dragon, he didn't know whether to design one dragon ... or some small elements 
of the different dragons, the combinations of the elements of the different dragons.

Interpreter, translating Aaron: When he draw the first part, the upper part, it's very difficult for him to figure out how he should design the second part, the bottom part to make the design, to make it harmonious, very balanced. So, I think it's the difficulty in his mind. How to make the composition a very balanced one.

Rebecca experienced similar difficulties. Her activities were mediated by a lack of confidence at the top level of the activity system when it came to trying something unusual. In order to try to overcome these problems a new theme, responding to teacher guidance, was evidenced in the division of labour.

\section{Theme two (2A): Responding to teacher guidance}

BS: Rebecca, can you tell me what was difficult for you?

Interpreter, translating Rebecca: So at the very beginning she was not confident enough, she was afraid, how do you say, she tried her first design by using a whole turtle with a turtle head and turtle body, and after you suggested her to use some, because she said that you saw some of her pictures with the baby [I had seen images on the mood board], you thought the picture was very good for using in the design, so she accepted your suggestion by replacing the turtle head with the baby head. Her difficulty, I think her difficulty is that she is not bold enough to try new things, because she thought that it is unbelievable to use a baby head on a turtle body.

Excerpts from the student reflections echo Rebecca's concerns, which reflected the inherent entanglement of primary, secondary and tertiary artefacts:

SR31: How to reproduce this image through modern design, I was at a loss. Thanks for teacher's guidance ... I found my way;

SR35: When I got the phoenix theme, I have no idea what to do, I think it so difficult, but fortunately [teacher's name used] told us a way;

SR36: I found it difficult and had no inspiration. I didn't have the confidence to finish it well . . . under the guidance of the teacher we started;

SR38: I'm very worry. Because I have no idea about water buffalo. [Teacher's name used] gave me a wonderful shape about the buffalo; 
SR53: It easy to say, but not to do. Then I lost aim . . . because I have so many [to] choose. I can't justice which one is better . . teacher give me some advice;

SR77: In the beginning I was at a loss, I don't know how to start this topic to design a $\operatorname{dog} . .$. . Brenda helps me the most.

From the student reflections just discussed, it is apparent that our guided teaching approach was well received. However, days requiring high levels of teacher involvement were exhausting as individual attention was provided to the students. I was constantly encouraging the students by drawing and demonstrating how to combine imaginary characters in the ideate stage, checking for originality and troubleshooting technical problems on their computers.

Although teacher guidance was sought, students also recognised a need for perseverance and recognised the need to commit to work in a self-directed way. This had been alluded to in the branding project, but here, there was an almost resigned or philosophical tone when students reflected on feeling good about making their own decisions about design process changes. However, feeling good, whilst recognisable as a secondary artefact, cannot be attributed as an object-oriented motive at this top level of the activity system because arguably the need to complete the project was the goal of the activities (Leont'ev, 1981):

SR46: I think everything is not easy, we must try hard for our work. If you haven't put yourself into your work. It will be a bad design;

SR55: There is no shortcut from beginning to completion ... you need to have your own ideas ... then check and modify the work;

SR82: The more you do, the more you choose, the better you will feel.

Notwithstanding the positive and learning developmental change that I observed within the adjusting to collaborative learning approaches theme, attempting to uncover negativities associated with collaborative exchange proved difficult. In trying to draw a response, this next excerpt from Aaron helped me to understand why students such as Will and Matt in the previous dyad had been reluctant to offer criticism about each other's work: 
Interpreter, translating Aaron: So he is mentioning that in China, it is not very common for students who are not very intimate to give suggestions to other students when they have some other suggestions in their mind. He thinks it's an offend to other students if you point out if something is not good, or if it is quite incompatible, so he suggest, we can, the student can give some suggestions, can tell the teachers first, and let the teacher decide whether to tell the student or not.

BS: That's interesting, I didn't know that. Would you say that's a cultural difference?

Interpreter to BS: Maybe, because we really do not point out other student, other our friend's weakness, if we are not very close friends, but if we if the common classmate we do not point out their weakness.

This finding is surprising because it is common practice in New Zealand classrooms for students to reflect on, and offer peer critique in either informal situations or formal presentation settings. Thus, a contradiction was found to exist between my expectations and the student's levels of critical willingness. My assumption was that if students did not engage with the practice of peer critique, beneficial learning opportunities would be limited. In Aaron's opinion, it would seem that any critical exchange between students would only become something of value once it has been filtered through the teacher. Interestingly, this finding supports some of the conversations I had been having with my colleague. We had both been surprised about the reticence students had towards offering advice to each other when we were in the vicinity and they were sitting side by side. Often, they would ask us to convey their specific ideas to the student sitting next to them.

Aaron provided a tantalising glimpse into previous (historic) and other teaching methods and practices when we discussed points of originality in the interview. His views echoed the third theme navigating local and imported learning practices which had been evidenced in the branding project:

BS: How is making original designs difficult? What would you normally do in other classes?

Interpreter, translating Aaron: In other classes which is taught by Chinese teachers they seldom get the chance to self-design the idea. While they are guided by the teacher to a specific way or do some exercises. 
BS: How are they guided?

Interpreter, translating Aaron: So, the teachers show some examples, or some illustrations to them, that let the students get the idea, and ask the students to do a very similar one, just like the example.

This intriguing assertion was surprising for me at the time as it was not anticipated. From the limited discussions I had with Chinese teachers, I assumed that teaching practices in Chinese classrooms were similar to those in our classes. However, I could not be sure of this because I had only ever walked past their classrooms, and often the doors were only slightly ajar. Three student reflections supported Aaron's assertions and provided more insight into the differences in teaching and learning approaches between our New Zealand classes and the students' previous learning in Chinese classes. Although it was not my intention to conduct a comparative study per se, these next excerpts further ignited my curiosity:

SR18: I have to say your class is very fun, it is far more different with Chinese education in China, we have to do everything that our teacher told us, and we have to do [it] the same way, only one way could be best, there is more other choice to do what we want to do, you always cheer us up to do what we thought, and we could try many things, and choose the best, and you come to give us some advice, and we still could tell you what we want to do;

SR37: This class has the biggest different focus on divergent thinking and not constrained by directional thinking or textbook knowledge;

SR87: The difference between the new curriculum concept and the traditional teaching model is that the new curriculum pays more attention to the individual's ability, the potential.

Further questioning about Chinese teachers' expectations appeared to support notions of independent learning, but it was apparent that time was an ongoing issue for the students:

Interpreter, translating Aaron: So, for Chinese teachers, they always give lectures, or give them demonstrations about the use of the software, and then give them a topic, and ask them to, to do a design, that is relevant to the topic, when class is over, they finish the assignment in the dormitory.

BS: How much time would they spend doing the design in the dormitory? 
Interpreter, translating Aaron: So, they have to spend a lot of time designing in the dormitory, for example if the teacher gives them lectures in the class on the first day, on the second day the students are required to hand in their assignments. It's very difficult for them to finish the design in time.

Aaron revealed his resistance to learning new Illustrator techniques, preferring to rely on his existing proficiency with Photoshop in his interests of expediency. This was similar to Matt's reliance on software he was familiar with such as Photoshop and the Image Trace technique discussed previously. It had been revealed that Photoshop is taught extensively in Chinese classrooms and so the software was familiar to the students. Aaron's problems could be attributed to a top-level tension, and thus a primary technological issue; however, I have interpreted the issue also as a middle level tension, as tensions arose between computer software and rules/time:

Interpreter, translating Aaron: Image trace, ok. He thought that was easier. So, when you taught them how to use Pathfinder, Shape builder something like that, because he was not familiar with this kind of tools, so he thought, it took a long time, and it's not very effective, using this tool.

Encountering Illustrator software problems was directly raised in 25 student reflections. Tensions focussed on unskilled use of software and the impact of this problem, on transmitting action. Goals of completion of a playing card illustration design for the Miao cultural project were perceived to be compromised when time and software skills were limited, for example:

SR02: I encountered many difficulties, for example, the software was not skilled enough, and I was not careful enough, so that my work was not good enough;

SR55: For me, computer technology is the main problem. Because it determines whether you can finish the work;

SR62: Many totems [motifs] cannot be continued because of the lack of proficiency in AI;

SR66: Not knowing how to use some of the functions on the software is the biggest obstacle; 
SR76: There are some problems in the use of AI. For example, the design that has just started is not smooth enough. It's not a way of teaching by the teacher.

The last reflection directly references the Image Trace problem which was encountered by the educators, and which has been discussed. The last reflection evidences a clear secondary artefact tension around lack of confidence which in turn potentially limits actions and activities.

An additional middle level tension to be discussed concerns colour selection.

Rebecca's assertions reflected the multitude of evidence presented at the beginning of this chapter in the first dyadic discussion:

\begin{abstract}
Interpreter, translating Rebecca: She's always using the complementary colours, so she thinks that complementary colours are very matching, so she likes using that kind of colour, but it makes the design boring, very dull, so after you helped her with the different colours with the several circles, she thought these colours are very beautiful, very harmonious in the design. So, she thinks the using of colours is a difficulty for her.
\end{abstract}

\title{
Theme four (4): Negotiating learning and family expectations
}

A completely new theme, negotiating learning and family expectations, was raised on a Saturday night towards the end of the project. At 5:48pm, I received a series of WeChat messages in English from Aaron. I am providing it mostly in full, owing to the nature of the exchange. The paragraph breaks indicate the separated WeChat messages:

Brenda, I'm sorry to disturb you. I am [Aaron]. I would like to apologise for the completion of my homework for the past two weeks. I know I am in a bad state of learning for two weeks. The quality of the work and work is also low. In fact, I am also very upset. Because I was arranged by my father and our school teacher to take part in about ten competitions, most of which are print advertisements. I slept for less than 5 hours every night for 3 consecutive weeks, which led to my little attention to school classes and bad spirits in class. I'd like to apologize to you.

I reviewed my homework yesterday, which is the picture of the dragon of spades. I think there are several problems. First, the problem of 
composition, generally speaking, the orientation of the dragon head should not be on the edge of the figure in the decorative painting. That will lead to the dispersion of the graphic skeleton. Two, the picture is too specific, I am too obsessed with the shape of the Miao dragon, ignoring the beauty and decoration of the pattern. I have consulted many patterns of playing cards in the past two days. I think I should not draw an overly concrete dragon, but should adopt the form and composition of decorative paintings.

I have redrawn a sketch for you to see. And I've been looking for some dragon decorative patterns to show you, I want to remake it, do a really good work, but I'm afraid there will be some tension at one weekend to finish it. But I really want to do my work well, so I would like to ask your permission. But I really want to do my work well, so I want to ask your approval, first is to remake my work. Second is a little time for me to delay to Wednesday morning. I will give my new work in A3 paper. Can you give me a chance?

Following is a photograph of a new sketch, and three examples of the 24 dragon reference images that Aaron found on the internet and forwarded to me (see Figure 7.4). This figure includes an image of Aaron's subsequent process in class.

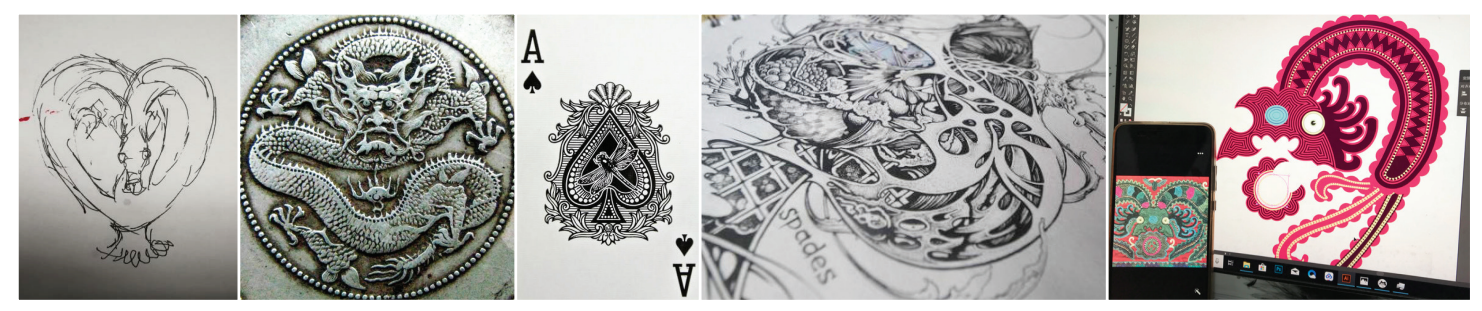

Figure 7.4. Aaron's new sketch, three examples of new references images, and his subsequent process in class.

I responded by expressing sorrow that the participant was tired, but offered the suggestion that competitions would help in learning to work quickly. After confirming everything that the participant requested was acceptable and offering a little more instruction on his new design direction, I asked the following question:

Can you tell me why your school teacher and father want you to enter so many competitions?

Because they want me to win more awards, which will help me to become a university teacher in the future. $:$

I want to be a really good teacher like you, like my father and my mother. 
Following a few more exchanges regarding the professional nature of Aaron's parents (both arts educators), I asked a final question:

What do you think about your parents' high expectations of you?

I think this is very correct, which helps me to make progress. Because no matter what extent I can achieve in the future, their demands are guiding and guiding me, whether I can get their demands. But if they don't ask for me, let me freely develop, I think I will do worse than now. :

People often do something worse than expected or expected. So I think it is certain that my parents are demanding me. Even if I do not have $100 \%$ to finish their requirements, if I do what they ask, I may be stronger than others who are less demanding of themselves.

Sure. This is just my personal view. :)

The use of the smiley emojis at the end of the messages were perhaps a reflection of Aaron's relief at having negotiated a change in his design direction for the project, or they may also indicate happiness about future prospects and family support. Managing family expectations and learning is something I have observed often with Chinese students in New Zealand; the pressure to perform and pass so they can move onto the next level of learning or into the workplace is considerable. However, this WeChat exchange exemplifies an attitude of acceptance and respect afforded to family expectations in regard to managing this tension.

Five months after this exchange and the project was completed, I received another series of WeChat messages from Aaron. He had entered his Miao dragon playing card design into a competition. His messages ran as follows:

Brenda, you directed my work "Miao embroidery dragon element playing card red heart 10" in China's provincial level association: Jiangsu Provincial Academy of Arts and Crafts, jointly sponsored "Jiangsu Art Design Competition 2018" and won the provincial award. This is a scanned copy, and there is also a certificate for your excellent instructor [see Figure 7.5]. I am currently awarded 87 awards at or above the provincial level (including international, national and provincial level awards) in the academic year 2017-2018. 

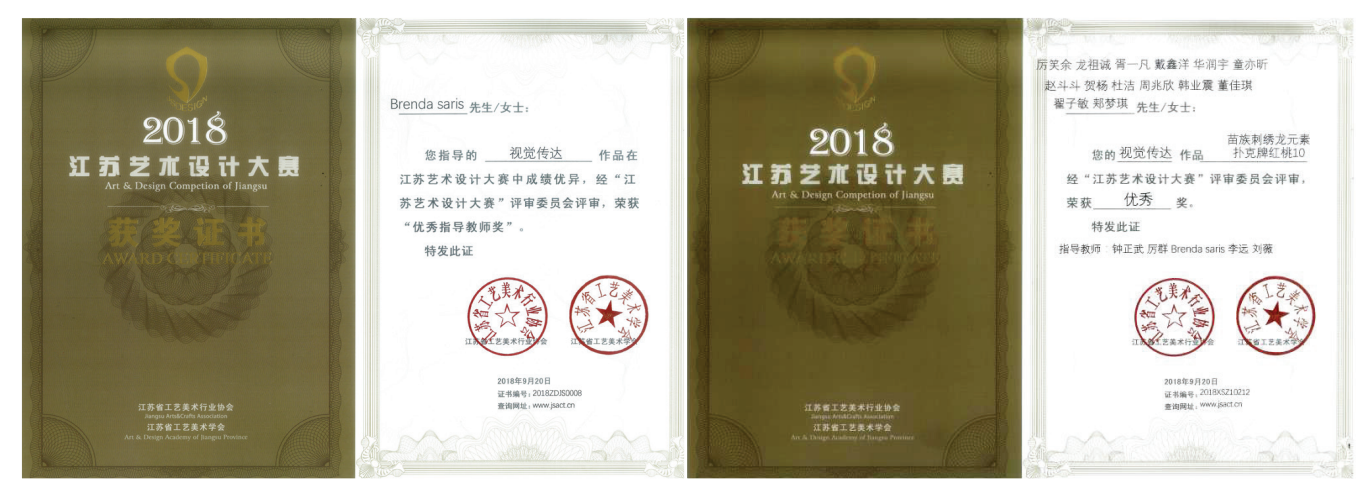

Figure 7.5. Competition certificates.

Aaron's success did appear to have come at great cost. Eighty-seven awards represented a significant number of competition entries, and his corresponding educational workload was put at risk. At the end of 2018, the message was relayed that Aaron had left the university in order to focus on his English IELTS learning. That the teacher received a certificate as I had made me question the value of competitions. What and how much value from competitions was placed on students by Chinese teachers and was this activity detrimental or advantageous? From an activity theory perspective there is an explanation. It is conceivable that the role of competitions may be viewed as a base level contradiction within a broader design education AS construction. To explain, Aaron, his parents and his Chinese teachers were guided by rules of the design discipline itself, and so their actions involved the questioning and evaluation of many opportunities. Object-oriented motives drove Aaron's attempts to consolidate a practice that extended beyond his classes and the university by entering competitions which was a process that drew on previous experiences of failure and/or success. This draws on Engeström's (2001) interpretation of object because other forms of teaching and learning experiences were present including navigating opinions of design judges who may come from the design profession. Similar to Connor's use of calligraphy in the branding project (see page 161), the three facets of the problem space can be explained: first, the object that was acted upon was a concept for a competition; second, there was a search by Aaron for a way to best represent the concept; and third, the desired outcome was reached when the concept won the competition, and so Aaron may eventually become known within the design profession as being successful (Foot, 2014). 
Three middle level tensions where artefacts (tiredness/too many competitions, anxiety, computer software, subjectivity) came into conflict with rules (time, software issues, and colour selection) were evidenced from this dyad interview. Moreover, all of the themes raised in the discussions permeate the middle level of this activity system along with the goal of the dyad activities being focused on completion. Aaron's WeChat message about the competitions highlighted the first middle level tension. His deployment of any number of artefacts was severely compromised by time. Despite his anxiety which I have interpreted as a secondary artefact, the rule associated with time allocated for VCD learning at the university was fixed and therefore required negotiation with the teacher. Happily, Aaron succeeded in meeting expectations when it came to handing in completed work at the conclusion of the project.

To conclude this section, it became apparent that there were some similarities between the findings from the first dyadic interaction between Will and Matt and this dyadic interaction between Aaron and Rebecca. But where Matt's activities with the Image Trace technique were interpreted as a top level tension, Aaron's difficulties evolved into a middle level tension due to time becoming a contributing factor. As I conducted this particular analysis, there were limited findings from Rebecca which may have been noted by the reader. Rebecca was rather shy as was mentioned at the beginning of this section; she worked diligently, and required much teacher encouragement. Aaron's level of English was superior as evidenced in the WeChat messages, and he was disengaged in the classroom, and thus limited interactions occurred between him and Rebecca, his dyadic partner. Therefore, when it came to the interviews, both Aaron and Rebecca contributed findings, and there was a focus on the lines of questioning rather than what had emerged on the video. The main point, however, about this particular dyad is that Aaron's frank assertions began to open up many more questions about his previous learning experiences and how those experiences may have been contributing or influencing his object-oriented motives in creative process learning. These points are further explored in the next chapter. Figure 7.6, presents key findings from Aaron and Rebecca. 


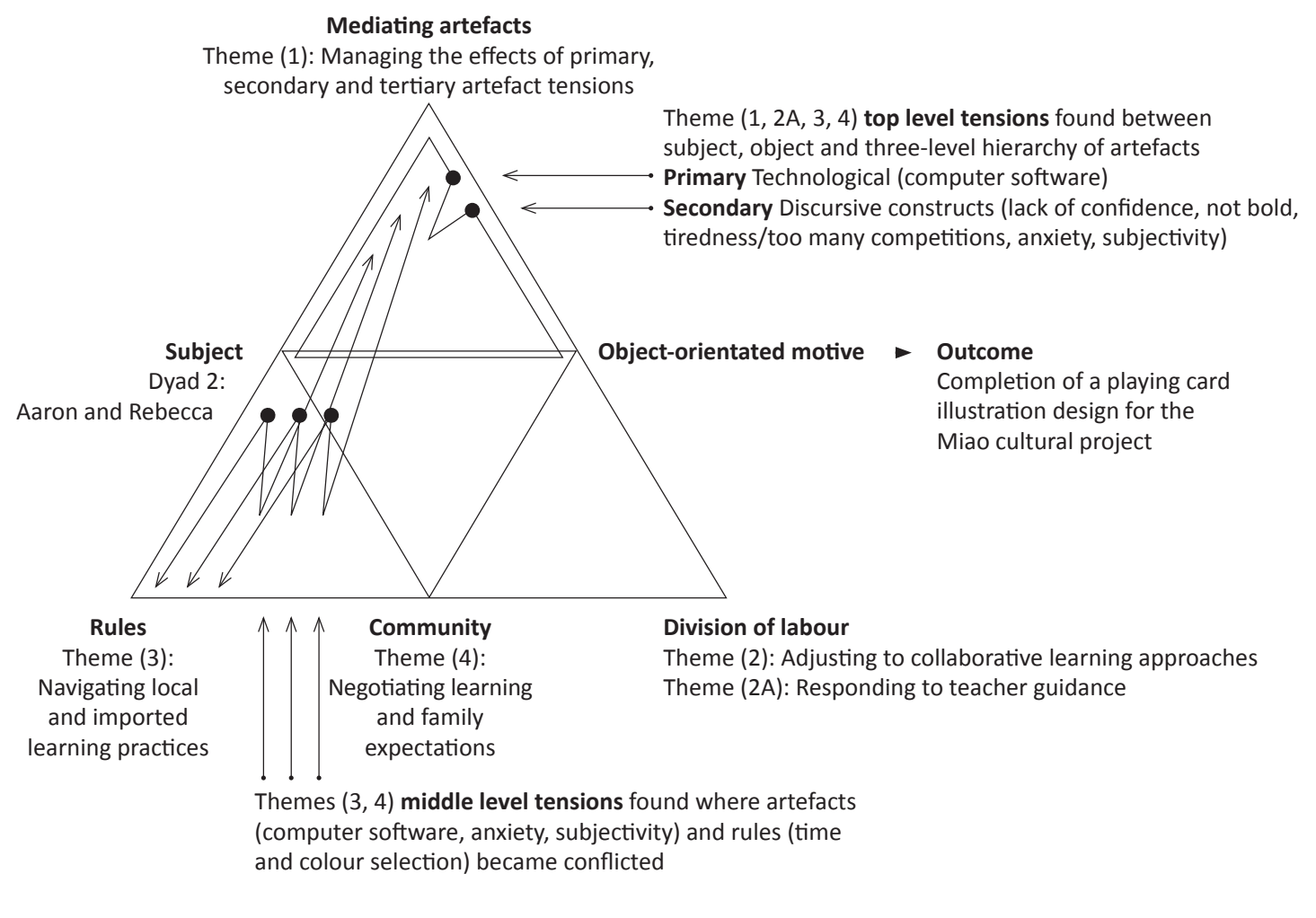

Figure 7.6. Dyad 2: Aaron and Rebecca, themes and tensions.

\section{Dyad 3: Nina and Jasmine}

The reader was previously introduced to Nina in chapter 5. Nina was the high achieving student who was involved in military training with freshman students in September 2017. She attended some of the classes in her military uniform, and at other times wore her physical education gear. She was physically very active and told me she played in the university football team. Her English was limited, but this did not hold back her ready sense of fun and mischievous smile. Like many of the dyad formations, Jasmine was the opposite of Nina, being quiet, shy, and hardworking.

The next excerpt illustrates a myriad of contradictory rules associated with local and imported learning practices that Nina, Jasmine and the other students attempted to navigate. Additionally, the dialogue reflected some of the aspects about Chinese teaching practices that Aaron had asserted:

Interpreter, translating Nina and Jasmine: So they mentioned the differences between the two, no the three [laughs] aspects, three sides. 
For the NZ teachers, they think the NZ teachers are more open, yeah, they let the student what they want to express, and the teachers are always be with them to guide them all through the whole course, and if the teachers want to make some change to their design they always, to make the change best on their idea, based on the student's idea. And for the [northern European] teachers, they are more serious, and more, how to say, it's very strict, strict with them, they pay more attention to very little details, and if the students have some of their own ideas, but the ideas is in contrast with, is in opposition to the [northern European] teacher's idea, the [northern European] teachers will stick to their ideas, and get the student to change according to their direction. While for the Chinese teachers, they taught the techniques and skills, the software first, and let the student to use their imagination to do whatever they want. It's not so, they will not guide them all the way through the design, only give them some suggestions, but not help them to make the change on the exact design.

The mention of Chinese teachers encouraging the students' imagination prompted me to ask the following:

BS: So, when they make an original design with the Chinese teacher, that becomes their own idea, completely their own design, is that what you mean?

Interpreter, translating Nina and Jasmine: So, they think for the Chinese teachers, they are more tolerant on the aspect of borrowing some elements from the other sources, just like from the internet, if you get some elements directly from the internet to use in your design, you know they, they don't care very much. They just let the student to have the design, but only if the student have their own, put some, put part of their own original ideas in it, its ok. They think its ok. They ideas is that the Chinese teachers are more tolerant in this aspect.

The response hinted at plagiarism and raised a number of questions for me. Why are the students allowed to copy elements from the internet and other sources? What is the purpose of this practice? Potentially the practice (or rule) is consistent with stereotypes associated with Chinese learning. However, my reading of the issue in this dialogue was conflicted because we asked the students to start their design process by sourcing reference images from the library or the internet. The aim was to use simple shapes in a considered way to reinterpret the Miao character or story for a playing card design by drawing on this research. This was done in the belief that there is still an aspect of creativity and originality involved in assembling a number of elements (shapes) into a 
cohesive design outcome. We talked about how it was different for the students to use the internet to find reference material for their character motif, but that they were not allowed to copy things directly from the internet or from their mood boards. Although I did not realise it at the time of the teaching, or in the participant interviews at the time, a very important finding was revealed when the transcripts were analysed. The use of the rough sketches (thumbnails) from our imported pedagogy in the design thinking ideate phase enabled relief for the students from the no-direct-copy rule that was imposed in the classroom. Although the dyad found it difficult to combine elements through sketching, at the same time they recognised that sketching could potentially trigger a developmental phase. This signalled a base level contradiction in the analysis where an introduced object of sketching triggered developmental learning:

Interpreter, translating Nina: It's very helpful for her future career because she learnt how to make an original design by using the pictures taken from the internet, or from the other sources.

BS: Can you explain that a little bit more? How did she apply the learning to this final design?

Interpreter, translating Nina: The way that she uses is to get the, get her favourite parts in the pictures and to select all the elements, her favourite elements from the pictures to combine them, and to hand draw them on the paper.

Interpreter, translating Jasmine: She thinks the way that you guided them all through the design is very useful, just like you ask them to search for the pictures first from the internet, or from other ways, and then to tell them to draw the favourite parts out by hand, and to ask them to combine different elements together to make the design. While Chinese teachers never do that.

Arguably, the real issue was less about plagiarism or copying and more that Nina and Jasmine felt that their Chinese teachers had a different way of applying or thinking about the practice of combining images found. Nina became quite emotional, exhibiting a love of process when I asked her how she felt about using thumbnail drawings or sketches to construct an original design: 
Interpreter, translating Nina: Very happy [laughs]

BS: Why does that make you happy?

Nina: [in English] It's my baby! It's my baby [hugs/covers her printed design on the table]

BS: It's your baby?

Nina: [in English] Yeah [laughing]. Because its mine!

With regard to the notion of sketching being an important part of a triggered developmental phase, student reflections supported similar degrees of excitement:

SR33: The teacher gave me a lot of opinions, letting me abandon some traditional traits, make some bold attempts and changes;

SR36: After many modifications and continuous progress, we finally saw a rough picture of the finished product. I was very excited because I had designed a piece of work by myself;

SR43: This course not only touches on new curriculum technology, but also . . taught me how to seek inspiration from some special directions and created my own design works.

Arguably, these prior excerpts revealed a motive that can be attributed to creative process activities whereby the iterative process led to increased ideation.

In summary of this dyad discussion, the revelation that sketching was a new way of working for this dyad was most surprising. It became apparent that it would be useful to understand more about Chinese classroom practices. The base level tension revealed by this dyad contributed to a change in the direction of my research. The idea that I should interview Chinese teachers rather than conduct a third case study in the classroom became apparent.

A diagram is presented that highlights the base level tension and key themes from the third dyadic interaction (see Figure 7.7): 


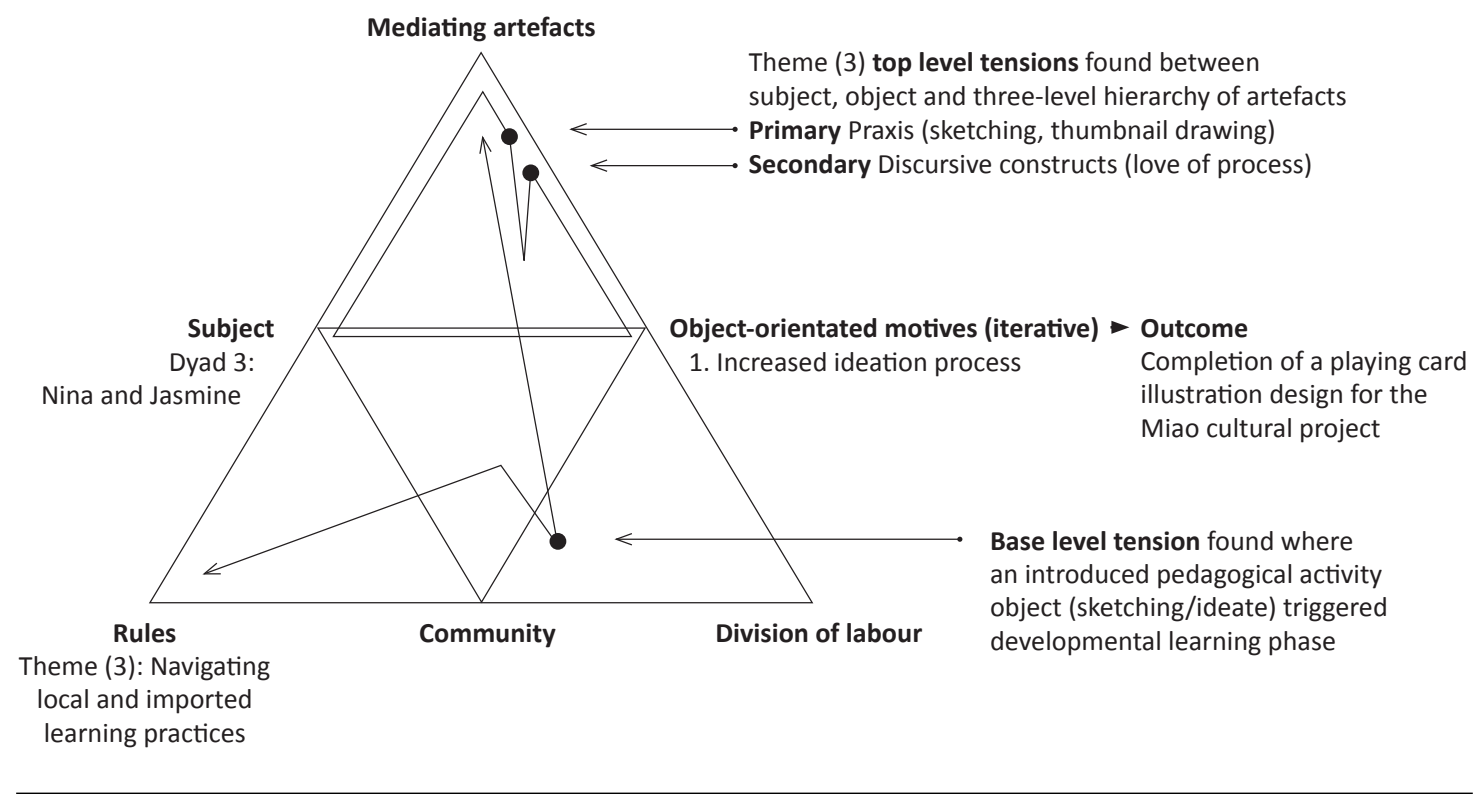

Figure 7.7. Dyad 3: Nina and Jasmine, themes and tensions.

\section{Dyad 4: Susan and Carl}

Inside the classroom, Susan and Carl hardly spoke a word to each other, nor did they interact much with their classmates. I got the impression that these two were high level achievers who were not easily distracted. An example of this was when they discussed the benefits of working collaboratively within the adjusting to collaborative learning approaches theme:

Interpreter, translating Susan and Carl: They talked about several points. The first one is if they cooperate with each other they can pick out more ideas, more suggestions to complement with each other. If they do it together they will get highly efficient and they'll work quicker, faster. Then next one, yeah is to help each other on the skills, on the technical operations of the computer.

Although the focus of this dyad was admirable, their assertions made above in the interviews contradicted not only my observations of them but moreover what was evidenced in the video footage as I did not see any verbal exchange or evidence of collaborating.

At one point another girl came and sat beside Susan and together they worked on aspects of Susan's work. This indicated that the work within the dyadic activity system 
extended to include others from the classroom community. In questioning how this was helpful for Susan, the discussion revealed the strength of reciprocal learning support that was available:

Interpreter, translating Susan: The body of the dragon is borrowed from some other students.

BS: Did somebody else draw that for you, or how did you borrow that?

Interpreter, translating Susan: Because, so while she was chatting with the other students, she talked about how she wants to make a design with the dragon body, the scales and the dog, with the dog head, so the other students said, he said, he draw the dragon before, so, the boy showed the drawing to her, yeah, so she could imitate the drawing.

The strength of the classroom community in these reciprocal exchanges cannot be underestimated. From the 100 reflections, almost half (45) of the students expressed thanks to their partner or someone else in the class, thus exemplifying the extent of collaborative learning. For example:

SR02: ... friends, they are working hard to help me;

SR06: My partner, he gave me some ideas when I had no idea, so that I could finish better;

SR12: My partner give me a big help, he always patient to help me solve the problem when I take a mistake he also can fix it;

SR15: . . my partner share me her pattern;

SR24: When I hit a bottleneck, I went to see what other students were doing, and they had elements or patterns that gave me other ideas;

SR27: ... my partner [name removed]. Thank you for your help and support, which made me feel very warm and happy;

SR35: Through communication with partner, make continuous improvements to my work, and make a big difference to my work.

In the analysis of the second dyad, Aaron raised a middle level tension whereby institutional rules around time came into conflict with three different artefacts, one of them being anxiety. A similar artefact in conflict with the middle level tension was revealed by this dyad. The issue of not enough time in relation to various secondary 
artefact issues such as anxiety or confidence was managed in part by exchanges made with peers outside classroom time. Susan and Carl described an organised dormitory culture where groups of four VCD students share a room and manage these issues by allocating time to meet each Sunday afternoon to help each other:

Interpreter, translating Carl: So they, the roommates, are also the classmates in his own class, so they assigned Saturday to be the day that they hang out, and they always do the assignment on Sunday evening.

Interpreter, translating Carl: The four boys always do the design together because they can exchange the ideas at the same time, but their working time varies according to different people. Some of the boys are very confident so they take less time, while the boys [who] are not so confident they took a long time.

The system that was described suggested that the practice of helping each other was something that had been established for some time. These next student reflections described this specific cultural project as particularly torturous, leaving me wondering if our imported teaching expectations were unrealistic.

SR11: My biggest difficulty is that I don't have enough time, including lack of time in class and out of class time. The worst part of classroom time is that I don't have enough time;

SR27: During these three weeks, we live on the front line of dormitory and classroom everyday;

SR41: The work was very hard and busy both the lesson only have three weeks' time, so I spend many time designing my work in my free time.

The motive of perfection raised in the middle reflection added support to the previous case study where perfection was found to be a motive. In this instance, time was compromising the students' desire to achieve perfection. As teachers, we were very aware of the long timetabled hours these students experienced. One night a week through this particular project, takeaway dinners would be delivered to the classrooms around $6 \mathrm{pm}$. The design class officially ended at $6.10 \mathrm{pm}$ and the spoken English class would begin at $7.30 \mathrm{pm}$. Amongst ourselves we aimed to not over-burden students by setting homework we knew would be difficult to complete after $6.10 \mathrm{pm}$ or $9.10 \mathrm{pm}$ classes or before early morning 8am starts. 
My colleagues and I were discussing originality in a similar way to how it had been discussed with Aaron (dyad 2) when the navigating local and imported learning practices theme arose:

Interpreter, translating Susan and Carl: They think that in other courses the teacher also ask them to make original design.

BS: That's quite different to what Aaron said.

Interpreter: Yeah, maybe they [have] different ideas, they think about the teaching methods differently. But he thinks he never met a design so good like this one.

Aaron had previously said that Chinese teachers seldom give students the chance to self-design the idea. I had interpreted this in respect to students seldom being given the opportunity to design in original ways in Chinese classrooms. It was a surprise to hear this dyad contradicting him. Aaron may have been referring to self-designing in regard to conceptualisation, and this dyad may have been referring to Chinese teachers limiting originality through the entire design process. Furthermore, Nina and Jasmine had asserted students were encouraged in the Chinese classroom to use their imagination. However, they suggested that the teachers did not guide them through their individual design process, and it appeared that there may have been a tolerance or acceptance of copying. Hence, conflicting opinions further highlighted a requirement to investigate Chinese teaching practices in order to understand how the students' experience of previous approaches may have influenced their practice in the New Zealand taught classrooms. The conflicting points that the dyads were making were important points, but they were difficult to understand.

Although it would appear a small number of findings were elicited from Susan and Carl, their interviews were revealing as they mostly supported or contradicted other dyadic findings. Specifically, the requirement for understanding what may have been helpful or indeed different for the students in the different (local and imported) classroom environments became more pressing for my research. The tensions that emerged from this dyad are presented in a diagram which depicts key findings from this fourth dyadic interaction (see Figure 7.8). 


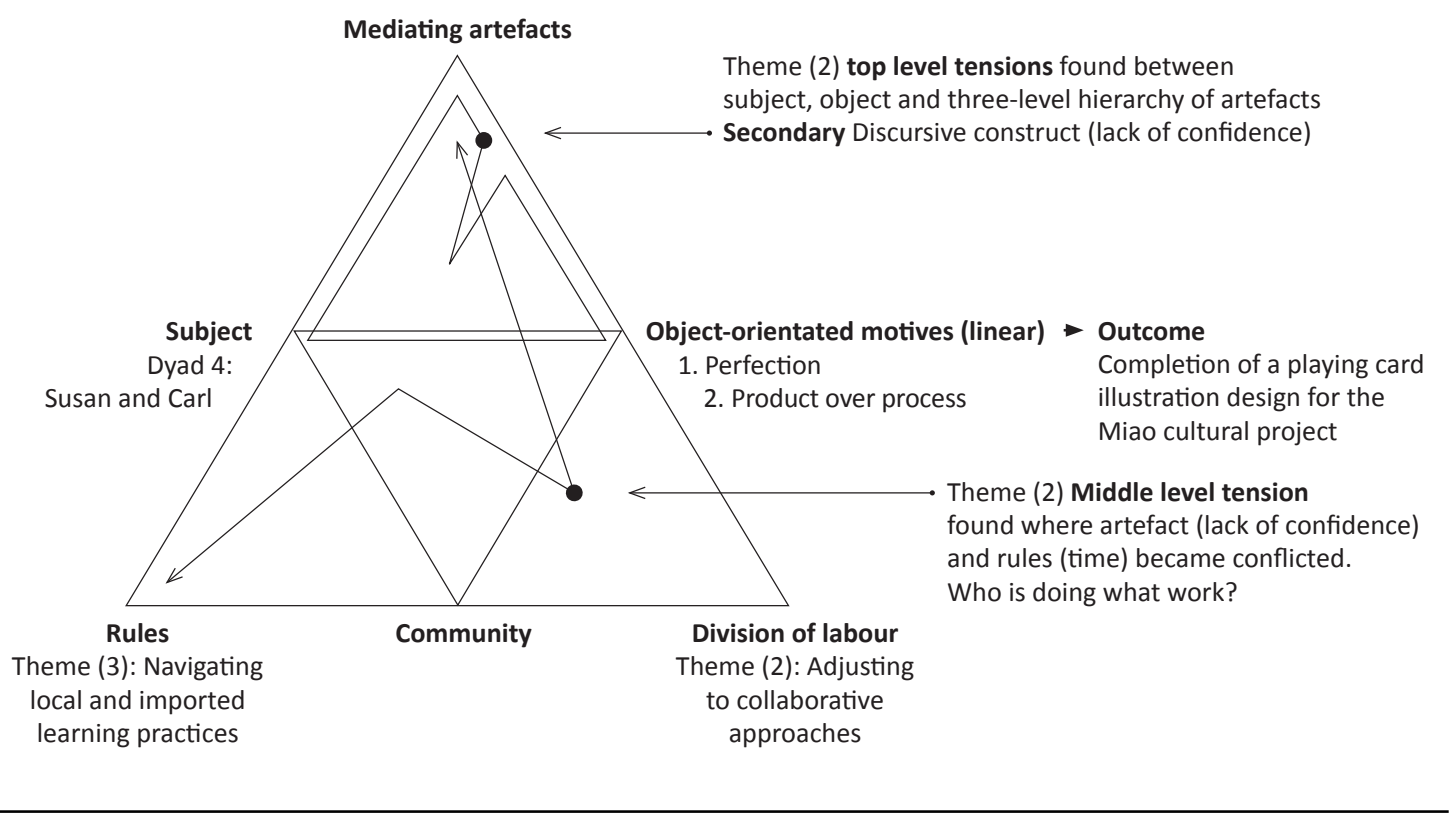

Figure 7.8. Dyad 4: Susan and Carl, theme and tensions.

\subsection{Discussion and conclusion}

From the analysis just discussed a figure is presented which compiles key findings from the four dyadic interactions and student reflections (see Figure 7.9). 


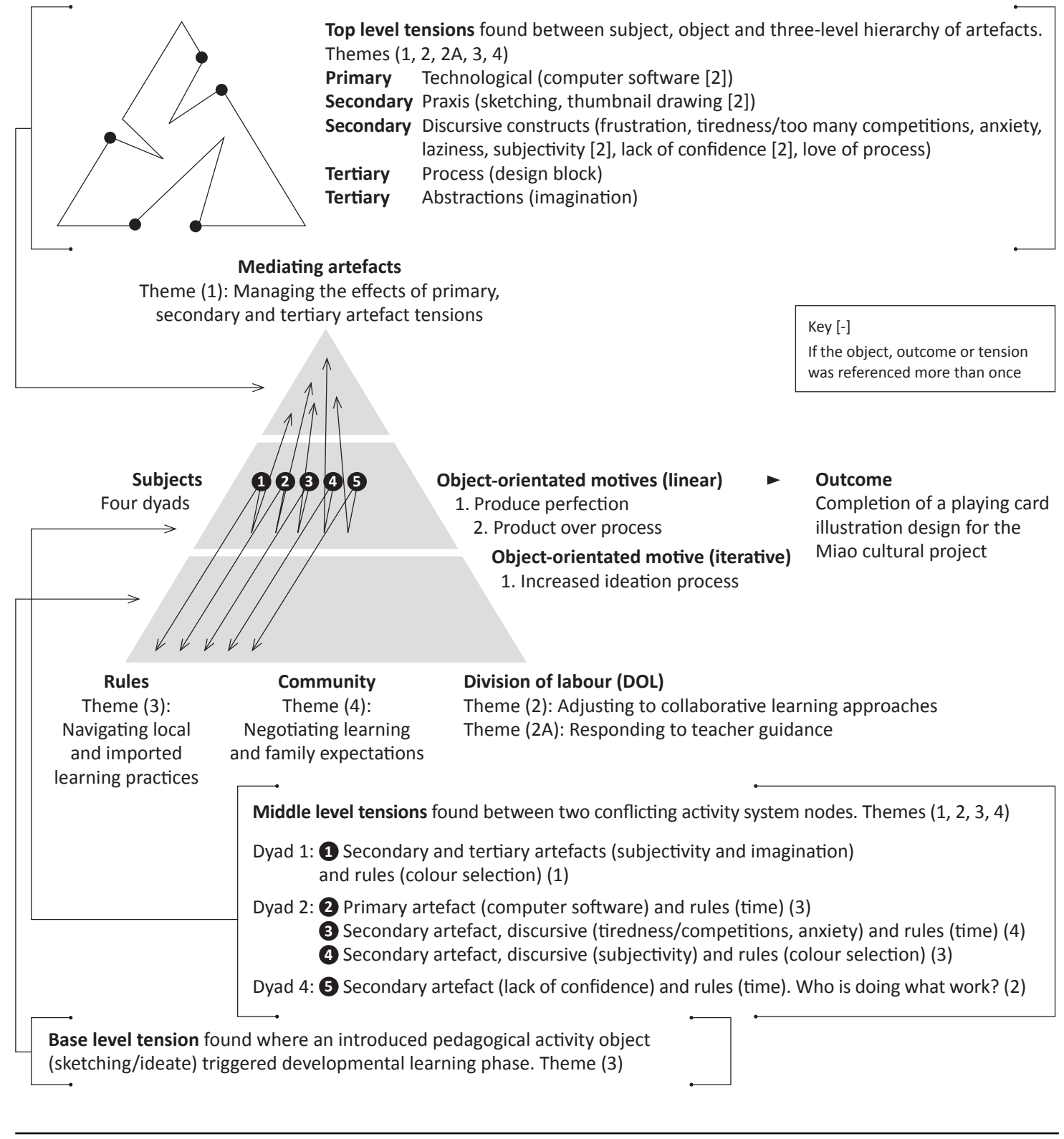

Figure 7.9. Miao cultural project: Key themes and findings from combined dyadic interactions, observations and reflections.

The focus of the analysis of the Miao cultural project was to further tease out how students navigated a different pedagogical approach by uncovering tensions and contractions that were experienced by four VCD student dyadic AS. The aim was to complement findings from the previous case study which focused in particular on the role of artefact mediation in the three-level hierarchy of artefacts. Overall, it was hoped a more thorough or rounded view of the dyadic creative design process phenomenon would be revealed by including another pedagogical project. 
Within the Miao cultural project case study, two new key themes were found from the combined dyadic activity systems, and tensions were linked to the new themes in the figure above (Figure 7.9). For the purposes of the discussion to follow, the two new themes have been added to the previous case study themes revealing that overall five main themes (linked to four AS nodes) were found from both case studies:

1. Mediating artefacts: Managing the effects of primary, secondary and tertiary artefact tensions;

2. Division of labour: Adjusting to collaborative learning approaches;

2A. Division of labour: Responding to teacher guidance;

3. Rules: Navigating local and imported learning practices;

4. Community: Negotiating learning and family expectations.

The first, second and third themes from the previous case study were again evidenced in this analysis. Adjusting to collaborative approaches seemed to evolve more naturally in this project, and from the reflections there was evidence that students shared or gave each other ideas and drawings, with almost half (45) thanking their partner or someone else in the class for their support. Within the division of labour the data also revealed a more direct and individual student exchange process in the work with the New Zealand teachers which led to the new theme, responding to teacher guidance. Rebecca first raised this theme when she was lost at the beginning of her process and six student reflections reiterated her concerns and appreciation for the guided teaching approach. The evidence was less apparent than in the first case study where motives involved product over process and perfection. Negotiating learning and family expectations a new theme that arose in the Miao project, was linked to community. A middle level tension relating to this is discussed further on.

Top level activity system tensions revealed five constant subject, object and three-level hierarchy of artefact tensions. At first glance, figure 7.9 appears to indicate that there was less replication of various top-level tensions that were referenced compared to the first case study. However, the intertwined nature of top-level AS tensions reflects the previous case study. The Illustrator tools were problematic, and despite drawing/sketching being useful to Nina and Jasmine, it created an issue for Aaron 
when he struggled to imagine which parts of the dragon to combine. The list of secondary artefact discursive constructs grew to include: frustration, tiredness/too many competitions, anxiety, and love of process. Furthermore, laziness, tiredness, subjectivity, lack of confidence and love of process were referenced by the dyads in this analysis; therefore, these tensions have been evidenced in both case studies. At this point in the research I did question Foot's (2014) theory of subject, object and mediating artefacts being kept in constant tension. Foot says the result of these constant first level tensions is expanded sequences and cycles of learning. However, rather than expanding learning, owing to the rather long list of secondary discursive mediating artefacts I was uncovering, it seemed the learning was restricted. These behavioral and emotional findings, for example, tiredness, may be viewed as blocking the ability for actions. However, arguably if goals of completion are strong, the ability to overcome object tensions such as tiredness is witnessed by the way the students accelerate their work and enthusiasm towards the end of the project. Furthermore, this expanded sequence included a temporal process aspect; time was simply running out for the students to complete the work. Therefore, Foot's theory of expanded sequences of learning is supported, and the idea of subject, object and mediating artifact analysis being kept in constant tension was indeed evidenced.

Middle level activity system tensions were two AS nodes that came into conflict (Engeström, 1993; Foot, 2014). The rules which stipulated classroom time allocations and the implications of these constraints on student activities were clearly evidenced by two of the dyads. One such example was the organised dormitory culture that Susan and Carl described where students came together to help each other most Sunday afternoons, which again led to the question, who is doing what work? Most notably, time was a large factor in Aaron's struggle with completion owing to the extensive amount of time he spent entering competitions to satisfy the wishes of his family. Furthermore, he mentioned a teacher who encouraged him to spend time on competitions. As such, this was something that indicated and supported the notion of further investigation involving Chinese teachers' opinions. Computer software and colour selection tensions with time also highlighted concerns for the students. Of the 100 reflections, 38 (or almost 40\%) students discussed their problems with colour selection, and Illustrator software problems were directly raised in 25 of the student 
reflections. Therefore, this case study overall has revealed that rules conflicting with the three-level hierarchy of artefacts were major areas of concern for the students.

One base level activity system tension was revealed when Nina revealed her love of process when she used the thumbnail sketches (an introduced pedagogical activity object) to trigger ideation. Unlike when Connor introduced calligraphy into his process in the last case study, the potential for Nina was realised because she recognised and enacted a developmental learning phase. Moreover, her assertion that this way of working, unlike her usual practice, evidenced a motive where use of an iterative process led to increased ideation. The joy of creating original works was palpable during the interview, and the no-direct copy rule associated with the imported teaching practice was relieved. Furthermore, Nina's implementation of sketches as a new way of working implicated Chinese learning where Chinese teachers appeared more tolerant of copying. This led to a questioning of the purpose of this practice as a potential stereotype associated with Chinese students. As was discussed, students began their design process by sourcing reference images from the library or the internet, but rather than copying the images they were used to inspire or inform the design thinking ideation phase. It can be argued that the New Zealand and Chinese teaching approaches were different, or perhaps they were similar, it was difficult to tell. This quandary, along with Aaron's experiences with competitions, changed the direction of the research following this case study. Eight Chinese teachers from the university were interviewed, and findings from the interviews are presented in the next chapter.

In conclusion of this chapter, one particular student reflection captured my attention. In my opinion, this seemingly simple reflection recognised the affordances of using an activity system to analyse student actions and activities:

SR91: In this class, I learned that design needs cooperation and constant trial to create better works.

'Constant trial' may be interpreted as fundamental tensions that exist between the subject, object and mediating artefacts (primary, secondary, and tertiary). 'Design 
needs cooperation' acknowledges the notion of rules, community, and division of labour working in a cyclical fashion for developmental learning, and thus for innovation to emerge (Foot, 2014). 


\section{Chapter 8. Hunan City University (HNCU) teachers' perspectives}

The students' perspectives about Chinese teaching and learning at HNCU, discussed in the previous two case studies, seemed to be in keeping with stereotypical assertions outlined in the literature review. It was evident from the findings, questions and themes that emerged from the two cases that prior learning in China was influencing learning in New Zealand classes, and that Chinese teachers were potentially one of the most important community groups that influenced enactment within the dyadic activity systems. I was left with the impression that Chinese teachers thought differently about design process learning and, although it appeared students were encouraged in the Chinese classroom to use their imagination, a tolerance of copying existed. This, in combination with assertions made in the literature about Confucian creative education being misunderstood, initiated the decision to conduct teacher interviews. It became clear that the research could be strengthened if forces related to prior learning and Chinese pedagogical practices were examined for a more comprehensive view to be presented (Li \& Wegerif, 2014).

\subsection{Analysis}

The aim of the interviews was to hear teachers' points of view, and rationale for their practices. The analysis of the teachers' interviews has been structured differently to the two previous case study analyses where the student dyads were examined individually and then combined for the discussion and conclusion. In this chapter I worked on the notion that Chinese teaching interventions, pedagogies and motives were located at a tension intersection point of a student's production pathway within the subjectartefact-object interaction (see Figure 8.1). 


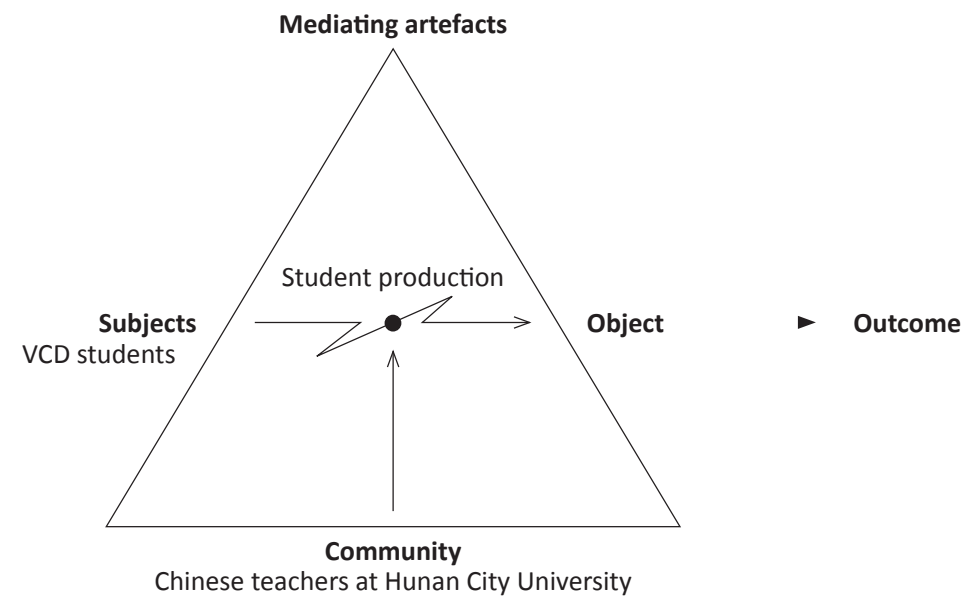

Figure 8.1. Tension intersection.

Eight teacher interviews were conducted at HNCU in China during a three-week period, from $10^{\text {th }}-28^{\text {th }}$ September 2018. Pseudonyms are used in place of actual teacher names for the analysis which follows. Traditional names were deliberately chosen for the pseudonyms, because none of the teachers referred to themselves with western oriented names; this was a more common practice amongst students. The teachers' names in alphabetical order were: Ang, Dong, Lei, Ming, Shi, Ting, Yin, and Zhu. Two interpreters were involved in these interviews, Hua and Shing. As was discussed in Chapter 5, I was involved with Hua from the beginning of the research and had travelled with her to Xiangxi to prepare for the Miao project. The differences between Hua's interpretations in the interviews, and the more candid interpretations from Shing were revealed in the teachers' interviews that follow. I interviewed participants Shi, Ting and Zhu with Shing, and as a result there is more emphasis on their discussions, along with dialogue from Lei who invited me into her classroom to observe students working.

Three main themes were found; the first relates to the overwhelming amount of data that discussed issues associated with reconciling imitation with imagination, a tertiary artefact. 


\subsection{Theme one: Reconciling imitation with imagination, a tertiary artefact}

This theme immediately spoke to top level activity system tensions where artefact use is driven by constant object oriented motives (Foot, 2014). Of the eight teacher participants, seven directly discussed the issue of copying or imitating. Much of the time the issue was raised without any prompting. This particular example from Shi was typical:

BS: Can you tell me, what is the most important thing that you want your students to learn?

Shi [in English]: The most important thing is the ability to think independently. You know Chinese students like to copy the other students if they find a good idea and the whole class mates may be far away.

Interpreter: Shi finds sometimes the students have the same ideas, they just copy from each other.

BS: How is this a problem do you think? Why is this a problem?

Shi [in English]: Maybe they are lazy, they don't want to think?

At this point I asked if we could talk about this idea of copying a little bit more by explaining that I had witnessed the practices of high school students at a training school in Changsha (discussed previously in Chapter 5). Shi could not elaborate on why she thought this prior learning made them 'lazy', or how the laborious practice of copying could perhaps be beneficial for their learning in design; rather, she seemed frustrated:

Shi [in English]: I don't like the whole classmate have the same idea, maybe when you drawing, you can learning the success for idea, but finally you must think according to your idea [reverts to Mandarin] . . .

Interpreter, translating Shi: She just give us some example. When the child is walking, when the child is very small, the child depends more on the mother to walk, but eventually the child should walk by themselves. So, the case is very similar here. We need others' experience, but we should get rid of other people's obstacles, we get rid of others' thinking.

BS: Why, should we get rid of other people's thinking, why should the students do this? 
Interpreter, translating Shi: So it's a contradiction, to get rid of other people's thinking, but at first we should define how to, what is copying. To what degree is this works belong to the copying, that is the question?

Shi's fascinating excerpt revealed three main points: first, students copy each other and they may do this because they are lazy; second, the importance of developmental learning; and third, evidence of a spectrum of acceptance when it came to copying. The spectrum of acceptance was clarified later on in the interview when Shi showed me examples of student work on her phone. Using Photoshop, students were encouraged to assemble various aspects of imagery found on the internet into what she considered to be an original design. In the next excerpt we discuss this work (see Figure 8.2):
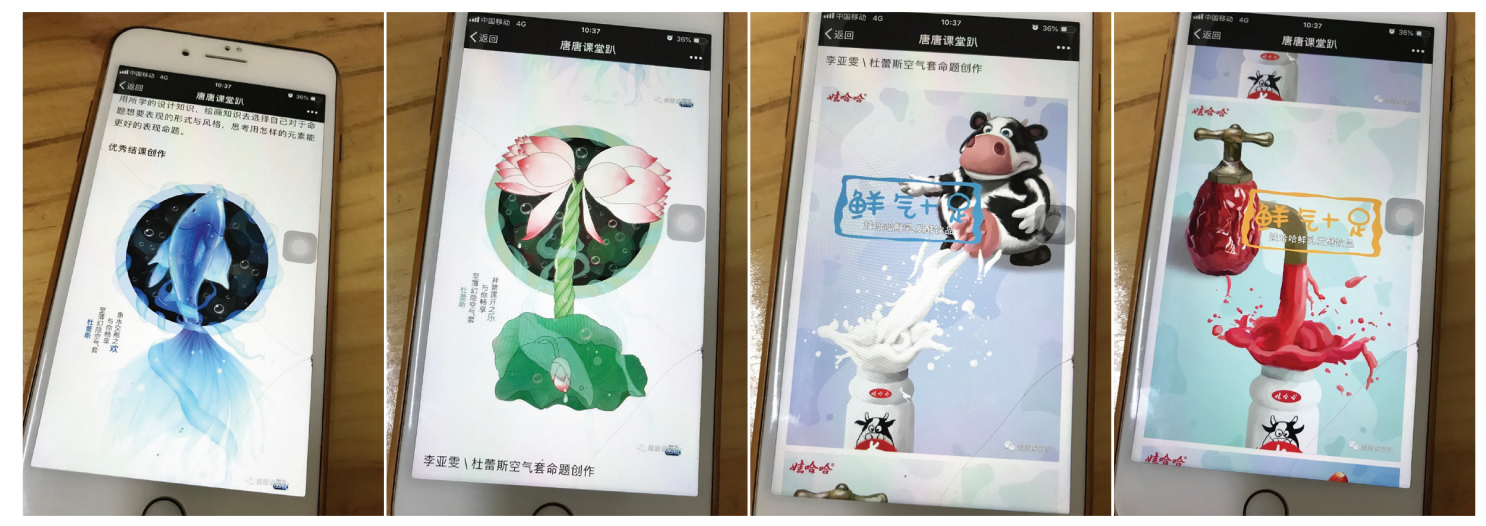

Figure 8.2. Student photoshop designs compiled from internet sourced images (author images).

Interpreter, translating Shi: She try to make the students change some parts, change some elements, the shapes, the colours, the composition, and to encourage the students to use their own mind, use their own imagination.

Shi [in English]: This is durex [a brand of condom also sold in China], this fish is copy.

BS: The fish is a copy?

Shi [in English]: Yes, just change the colour and the tail, and get some more smooth and back into the water. And this is the lotus, yes, the water lily. And this is a milk drink. They want, they want to show the whole fresh, the fresh milk, you maybe saw the cow in the website. [laughs]

BS: So if I went onto a website, I would find that picture of that cow?

Shi [in English]: Yes, but, but they change the drawing style. 
BS: This concept has come from the internet but the style has changed?

Shi [in English]: Yes, so you don't know this shape is copy from the website, but you see the whole picture you don't know.

BS: What is this one for, juice, fresh juice?

Shi [in English]: Yes, fresh juice squeezing the plum into the juice.

BS: So what is from the internet, is the tap from the internet?

Shi [in English]: Yes, the tap yes.

BS: Is the berry from the internet?

Shi [in English]: Yes.

BS: But the concept of the juice coming out of the tap, is that from the internet or is that the student's concept?

Interpreter, translating Shi: Also from the internet. From a film. Also from some advertisement.

Although Shi stated at the beginning of her interview that she did not agree with copying, she was in some ways promoting it. This was evident in the way imagery such as the goldfish was found on the internet and manipulated by Photoshop, and in the revelation that the concept had been found elsewhere. Arguably it may not be practical to photograph the goldfish and the plum to use as source images, and so it may be expedient to locate images from the internet and manipulate them with Photoshop to learn about software functionality. However, the lotus and the cow are illustrations, and are arguably derived from the imagination, thus the boundaries of acceptability become blurred. By extension, there can be no argument that a concept derives from the imagination, thus the spectrum of acceptability stretches. I did a reverse image search on both Google and Baidu for all of these images on my return to New Zealand. Although I was unable to locate exactly the same image or component parts, there were many humorous cartoons of cows similar to the depiction that has been discussed, and various photographs of taps pouring different coloured fruit juices. Failure to find an exact match may have been limited because I was using New Zealand search engines, and was blocked from Chinese websites. But, my failure to locate the exact images also reflects Shi's original question "to what degree is this works belong to the copying, that is the question?" 
Like Shi, Lei had been teaching for five years, and both her and Shi were responsible for teaching freshman (first year), sophomore (second year) and third year students. It is interesting to note they both discussed the dilemma of reconciling imitation with imagination in similar ways. The difference with Lei is that she was less philosophical about what constituted copying and more convinced about the benefits of copying and imitating for creative learning:

BS: How do you think your students use creativity in their design work?

Interpreter, translating Lei: Several points. The first one, she asks the students to emphasise the research, just like searching on the internet, search the information on the internet, the second point, she asks them to improve their skills, though imitating and copying, and third point, through copying and imitating to promote students' aesthetic ability, maybe this kind of ability can stimulate their inspiration. And the fourth point is the communication, a lot of communication with other students or with teachers, and the fifth is to ask the students to think out their own ideas first, and then she will start with the students' ideas to make it more, make it deeper:

BS: Make the ideas deeper?

Interpreter: Yeah make them deeper or develop it.

BS: Ok so those her steps, that's her teaching process. Can I go back to some of those earlier steps? I'm intrigued to know why she said that maybe imitating might work, and she mentioned skill, so could she talk about that more. What is she trying to achieve when students imitate, how does she think they can learn from that part of the process?

Interpreter, translating Lei: The first, she took poster design as an example, because the students at the very beginning have no idea of how to design a poster. They know nothing about it, so through the imitating and copying from some other references they know how to build the balance between the typography and the layout, something like that, the colour, they can do their own poster with a sense of aesathicisms. [deliberate mis-spelling]

BS: Aesthetic, the look of it?

Interpreter: Yeah they know how to appreciate it, and they will improve their ability as well.

BS: How does that improve their design ability? 
Interpreter, translating Lei: Because they know how to build the balance on the poster, how to choose the typography, how to lay out the whole poster, they can learn a lot, to improve their abilities in design poster.

Many of Lei's assertions made above became clear during a class observation. I joined her first year class on a Monday afternoon. This was the very first design major class for these students who entered HNCU at the beginning of the academic year. Thirtyfour students were working on desktop computers supplied by the university. They were using CS6 Illustrator (ai.), and textbooks were scattered around the desks, some open and in use. Lei expertly taught the tools and techniques of the Illustrator software by demonstrating the Pen Tool, Pathfinder, Gradient, Eye Dropper tools simultaneously. The students had to choose from nine options to complete six copied natural gas logo designs. Lei showed the students how to build one logo, and then deconstruct and reconstruct other versions at speed. I watched this demonstration, marvelling at Lei's ability as it surpassed anything I had witnessed in my years of New Zealand teaching. I was also astounded at the high expectations placed on these first year students on their first day of design learning (see Figure 8.3).

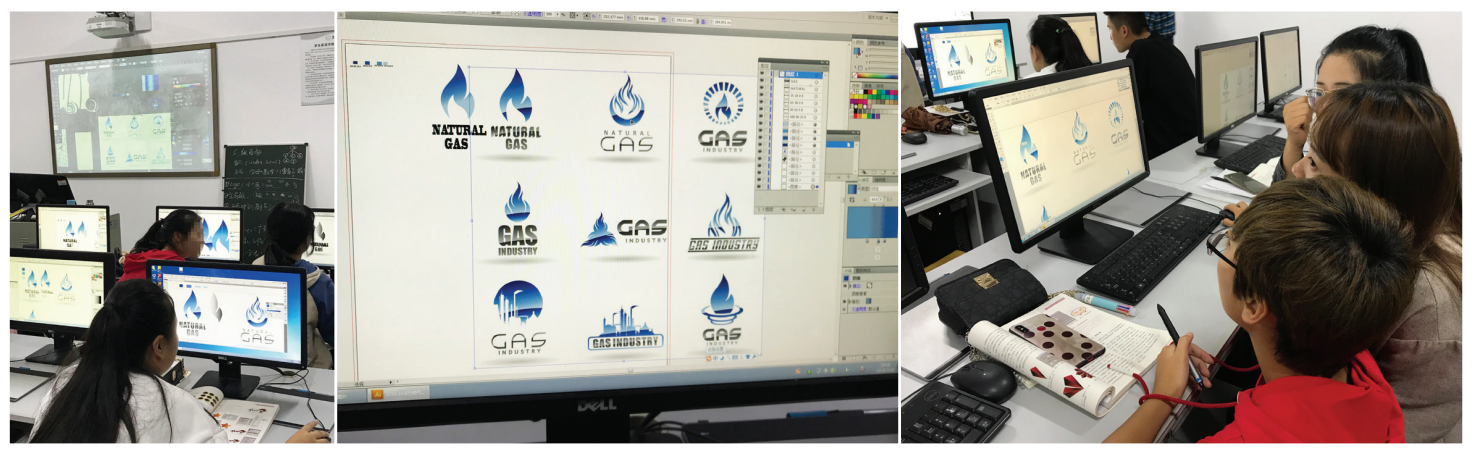

Figure 8.3. Software skills learning, natural gas logo designs (author images).

The following day, I observed a very similar teaching and learning process. However, this time, the students were copying poster designs from a number of Bauhaus artists. From 32 options, the students had to choose four designs and using illustrator software recreate copied versions of the designs (see Figure 8.4). 


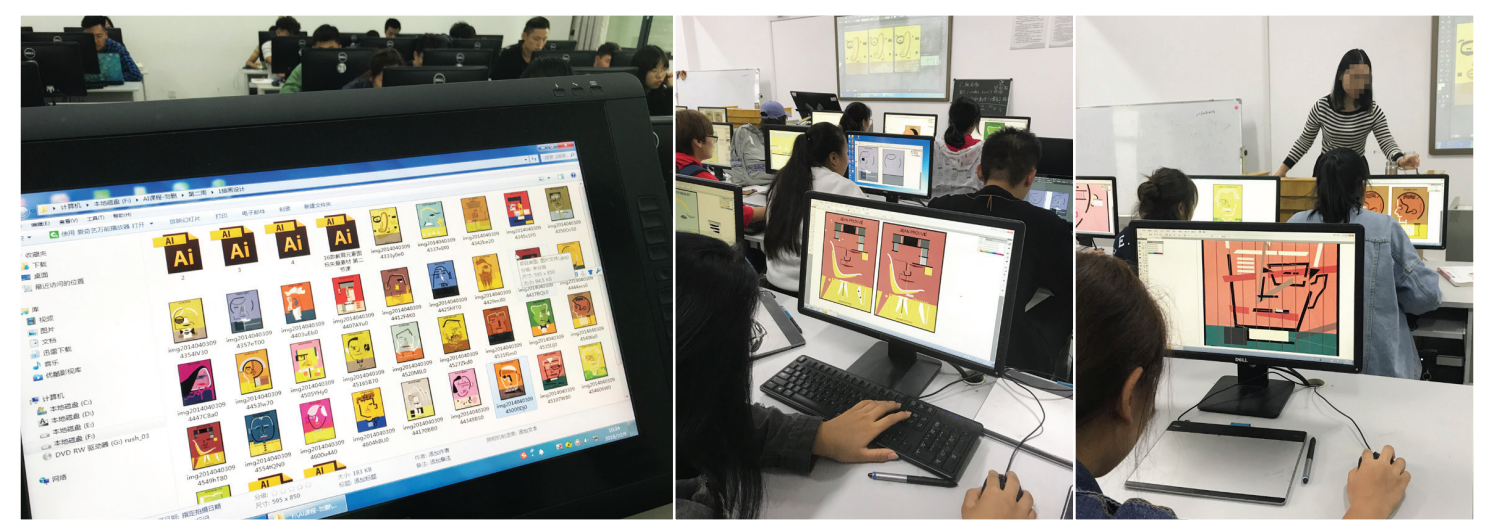

Figure 8.4. Software skills learning, Bauhaus poster designs (author images).

At the time of Lei's interview, it was unclear how she thought copying and imitation could lead to an acquisition of creative learning. After the classroom observations, it still was not overly clear as I had witnessed extensive software learning, and this learning was being facilitated by copying the gas logos and Bauhaus artist posters. Lei admitted she struggled with the gap between technical learning and creative learning, referring to this gap as a combination of the software and ideas:

BS: Do you experience any difficulties or challenges or problems in the way you have to prepare for classes?

Interpreter, translating Lei: The difficulty for her I think is about the nervousness. From several aspects, the first involves the teaching contents, because when she is starting a new course, she will collect all the information, all the references about the new course, it took a lot of time to collect all the references. That's one thing she is worried about, and the second point ... she is worried that the students cannot finish the project with high quality ... the students cannot meet her high standards. The third point that she is nervous is how to update the newest design ideas, because after she learnt so many software she said some names ...

BS: I heard her say PS and AI.

Interpreter, translating Lei: Yes, she learnt how to use them but it's hard to update the newest ideas, design ideas, she only know how to use the software but she cannot get a very good command of the ... I think she cannot achieve a good combination of software and the ideas together. That's the thing she is worried about.

After I had left China, Lei sent me examples of student work from the first year class I had observed (see Figure 8.5 ). She had talked about running an environmental project 
highlighting the negative effects of smog pollution with the students towards the end of her course.

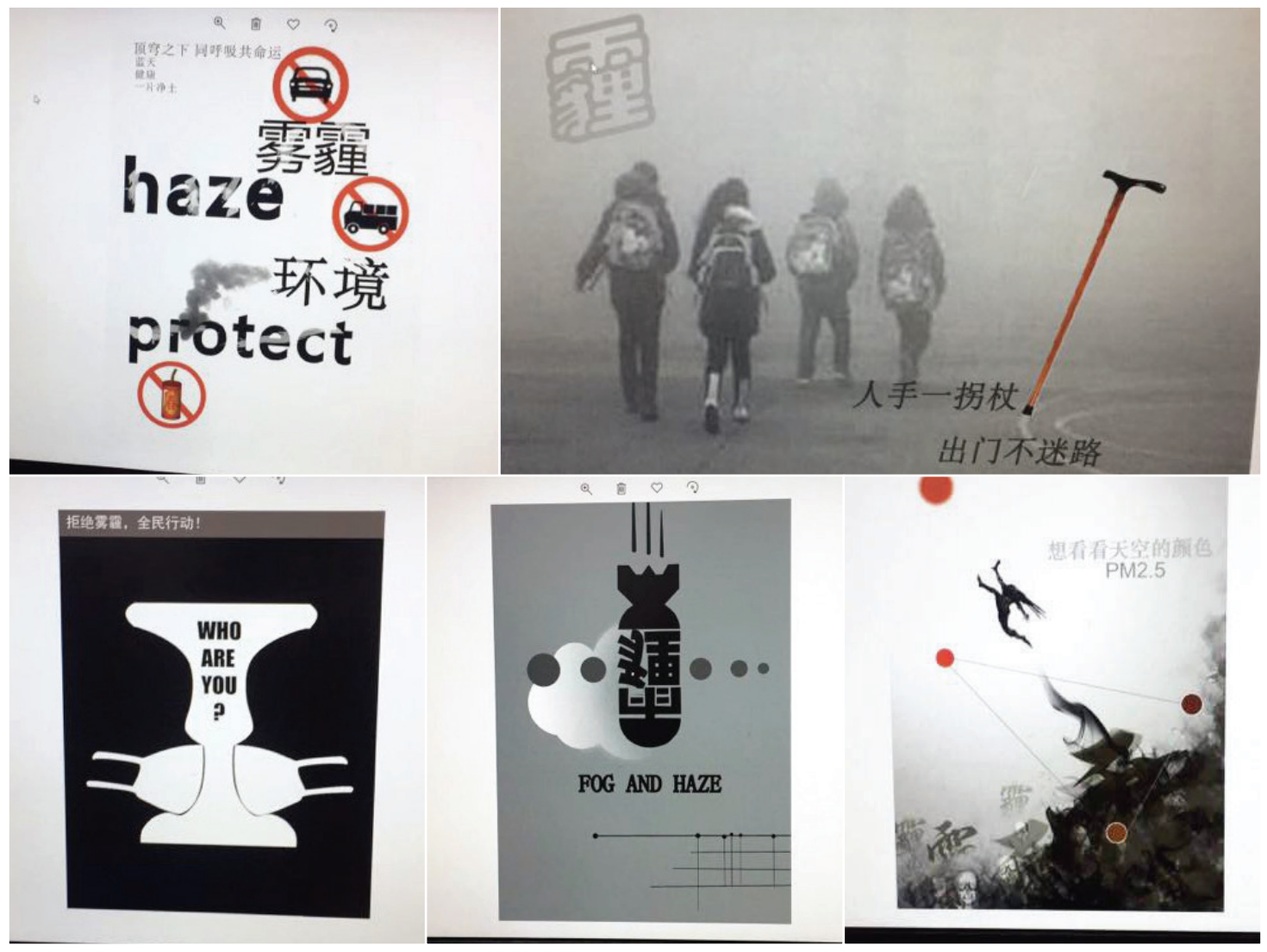

Figure 8.5. Smog posters received from Lei (participant images).

The photographs were accompanied by this WeChat message:

Lei [in English]: In my opinion, the quality of software learning is: students' proficiency in soft use; secondly, they can know how to make their thinking by seeing the works. Thirdly, students' visual aesthetics can be cultivated in copying logo, illustration, UI and other works. Students' learning attitude can be reflected in the analysis of methods and overall grasp of the overall work in the process of copying.

In light of Shi's interview and her examples where students used Photoshop to create a design based on an assemblage of images found from the internet, it is likely that the posters from Lei were created in the same manner. Ascertaining what was original and what was not, points to these two teachers of similar age, gender and teaching experience sharing a similar view of the acceptability of imitation. The bottom lefthand smog poster in Figure 8.6 exemplifies the most obvious example of imitation. 
Face masks have been positioned on Rubin's vase, an optical illusion developed by Danish psychologist Edgar Rubin in 1915 (see Figure 8.6). The discovery of perceptual ambiguities such as this influenced artists of the time who experimented with background and foreground, positive and negative space.

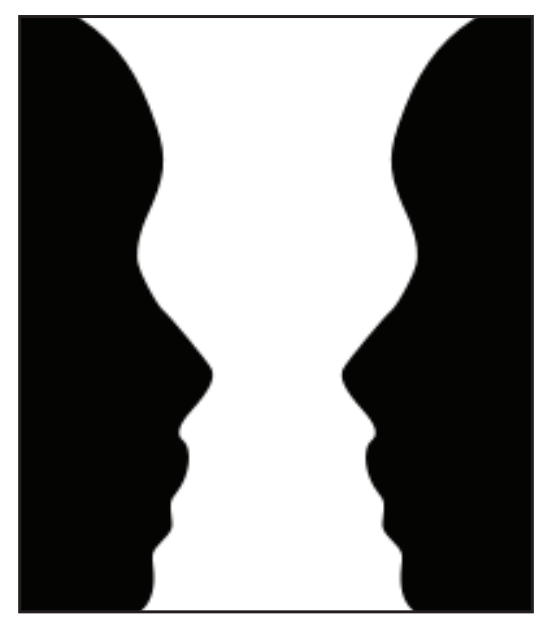

Figure 8.6. A Rubin vase. Retrieved from https://www.newworldencyclopedia.org/entry/rubin-vase

Two male teachers shared the views of the female teachers just discussed, but the way they discussed copying was more black and white as they suggested there were rules associated with the practice:

Interpreter, translating Zhu: yes norms some rules, and some points that the students should pay attention in the designing. Such as don't have the same idea as others, yeah, don't be the same with other works, especially the important or the famous works.

Interpreter, translating Ming: For the references the students get from the internet, he says they can use the images or the references as a guide for the students. And the guidance but they cannot copy it directly. They should have their own interpretations otherwise they will be punished.

BS: How are they punished?

Interpreter, translating Ming: For the design, for the course design if some students copy some images it's not as serious as the graduation project. If they copy for their graduation project they fail it.

In the following excerpt, the tensions surrounding the transformation from imitation to imagination were raised by Ming in association with my 'New Zealand' teaching. It 
was as though he sought a formulaic approach for teaching creativity, almost like the unlocking of some kind of 'magic formula' in order to teach creative processes, in the same way applied skills are taught. In his opinion, there was a contradiction between teaching creativity which was the requirement of the university and industry needs. Zhu also admitted to the difficulties he encountered when it came to enabling the transformation of student creativity:

BS: How do you think your students use creativity in their design-work?

Interpreter, translating Ming: This is a problem for Ming. He wants to have to talk with you on how to improve students' creativity while at the same time their skills based on their, how do you say . . applicable . . . because they will do very practical projects after they graduate, so based on the practical skills they have learnt and at the same time they have to improve their creativity.

Interpreter, translating Ming: He still thinks there is a contradiction between the creativity and our universities' requirement for cultivating applied talents, applied students.

BS: How difficult is it to shift them from imitation to using their imagination?

Interpreter, translating Zhu: He thinks your teaching is better in developing the students' innovation ability, and to him, he think it's sometimes difficult, it's a challenge to develop the students' creative or innovative thinking.

At the time of these participant interviews, in order to gain a better understanding about the effects of imitation on imagination which was widely discussed, I again referred to my visit to the training school in Changsha when interviewing Ang and Zhu. The purpose of my visit was to try to explain how and why this historic trajectory of learning appeared problematic for pedagogical practices at HNCU. Ang and Zhu expressed similar views about training schools which follow:

BS: How useful do you think that training is for students that come to this university?

Interpreter, translating Ang: For the training of the students in the high school, she says it is a shortcut for them to, to because they have to pass the college entrance examination so it is a shortcut for them to pass the exam by imitating pictures. 
BS: Why is it a short cut?

Interpreter, translating Ang: Because it's an easy way to learn from others' examples, because copying they can easier to catch the techniques very quickly by imitating others.

BS: Wow, I think that's very difficult [everyone laughs].

Interpreter, translating Ang: Because the students needn't use their own ideas to paint the person or the objects, by using their own, how to say, using their own ideas.

BS: So when the students are freshman students, how difficult is it to work with those students because that is all they have done up to that point? How does she shift them from imitating to thinking for themselves?

Interpreter, translating Ang: Maybe it's hard for the students at the very beginning because it's a transformation from the copying to the creating, but she also thinks it depends on the teachers that taught them.

BS: Zhu, how useful is that type of learning when they come to university? Interpreter, translating Zhu: He doesn't think he's right, but in his opinion, he thinks the imitation has two sides. The advantage is to train the students or to develop the students' drawing skills, but the disadvantage is to limit the students' imagination and creativity.

BS: Why doesn't he think his opinion is right about that?

Interpreter, translating Zhu: He's not sure he's right

BS: But that's what he thinks?

Interpreter: Yes.

It is clear the tensions surrounding the reconciliation of imitation with imagination is complex. Whilst the teachers claim they do not condone imitation, and they dislike the previous learning undertaken inside training schools at the high school level, it is in actual fact a common and daily part of teaching and learning practice. It is clear that the teachers recognise the creativity issue but are unsure about promoting creative pathways. There is a gap between successfully teaching both software and applied skills and creative innovation and thinking. There is a genuine concern that this needs to be addressed. Zhu tried to help students in other ways:

Interpreter, translating Zhu: So in his first lesson of this course, he will ask the students to do some research from the website to find out the 10 best 
logos designs, and ask them the advantage of the 10 best ones, in terms of colour, the graphs, or the shapes.

BS: The graphics?

Interpreter, translating Zhu: Yes the graphics and then the shapes, And then tell them the theories, the relevant theories and ask the students to draw, and sometimes imitate .. this is one part of the daily performance . . he ask the student to choose the ten best logo design and ask them to choose, to imitate, to draw. This can develop the students' appreciation ability and the choosing ability.

Who can say this is not a plausible teaching approach? Activity theory asserts that tools which stem from a user's cultural-historical origin extend human activity; therefore, arguably using tools to imitate promotes learning through time (Leont'ev, 1977). This is recognised by the Chinese teachers; Shi talked about teaching a child to walk. But copying alone does not allow for anything new or innovative to be created. The teachers are exceptional at teaching technical skills; for example, this software tool can be used to fill a square with colour. However, teaching a student where to place or orient a square within a 2-dimensional space without any explicit guidance or direct copying of an exemplar was not evidenced. Various theories were alluded to by the participants, but they were non-specific about what the theories were. One such example for placing a square within a composition could be according to gestalt principles, whereby objects may be grouped according to similar characteristics such as colour, but none of the eight participants mentioned this. It is likely that language barriers may have contributed to a lack of information about this being forthcoming.

\subsection{Theme two: Encouraging cultural and social rules of perfection}

This theme emerged from data around discussions relating to motives of process, product over process and excellence or perfection. Cultural and social rules of perfection are reflective of the way perfection, morality and hierarchy in learning were described in the literature (Confucius, 1954; Hung, 2016). Tertiary artefacts (process and abstractions) were implicated and, as a consequence, a middle level activity system tension was found when these tertiary artefacts conflicted with a rule where 
competitions are used by teachers in the learning curriculum. To recap, middle level tensions are encountered when two nodes of an AS conflict with each other.

\section{Process, or product over process?}

VCD student process was a constant topic of discussion amongst the foreign teachers.

One such discussion that took place between me in the role of teacher (New Zealand teacher) and a colleague (Northern European teacher) whilst teaching at HNCU was particularly telling. We were having a break in the staff room set aside for foreign teachers, and my colleague was feeling particularly frustrated and upset. I asked him why he was so frustrated:

Northern European teacher in conversation: Because, they just do it and don't really learn it in the understanding of what we think is learning, to analyse it and to reflect on it. They are learning only steps, and when they forget one step of the whole process, they dismiss, they are lost, totally lost in the whole context, the whole process.

Following this exchange I asked my New Zealand colleague how she was feeling about the students she was teaching:

New Zealand teaching colleague in conversation: Some days you leave the class feeling energised, after reaching a breakthrough with a student or devising an exercise which increases class energies and excitement. Other days I am completely exhausted, balancing between students who require a high level of direction and others who require a great degree of motivation. Generally students are polite and courteous. They are grateful for one-onone guidance, though they seldom challenge your suggestions and instead take them as direct instruction.

My colleague's response prompted me to ask the direct research question. How do you think the students enact and engage with creative process learning?

In my experience, students have the technical proficiency to follow steps introduced in class; however, on the whole, students are unable to connect the relevance in what's been taught in their own work. After spending time explaining and demonstrating new ways of doing, many students revert back to processes they are familiar with. Students also find it difficult to 
see how artist models can be used to inform or stimulate original thought processes. Rather, artist models are often copied directly.

Students also seek clear step-by-step direction and are reluctant to experiment with creative possibilities. In order to get students to engage in an iterative design process, I often have to introduce it as a stand-alone task, such as create three different colour versions of your character. Students have a tendency of needing to know what the final outcome is expected of them, rather than a willingness to allow the process to guide their work.

Many of the Chinese teachers advocated process as a way to guide open-ended possibilities, yet perfection defined as faultless through actions or process of improvement was the preferred indicator of a good outcome:

Interpreter, translating Yin: Only after accumulation of many experiments the students can achieve a final result, so students have to do the design by themselves. Many, many experiments they can get the final perfect one.

BS: How important is the final result?

Interpreter, translating Dong: Even though the final result is very important, he took more emphasis on the process. He thinks that process, he always ask the students to show the evidence of how the work improved, developed, so he thinks the process is more important.

Interpreter, translating Ming: So the final result is not his first priority. $\mathrm{He}$ put emphasis on the methods and the students views during their designing process. So he thinks that the methods and the process are very important.

BS: When Ming is marking the students work, what is the main criteria he is looking for? What is the most important thing?

Interpreter, translating Ming: He thinks the final result is not very important, but the process is his priority.

BS: So that would be his main criteria?

Interpreter: Yes.

BS: Does he have a percentage when he marks? What is process worth, and what is the end result worth? What are the [institutional] rules around this?

Interpreter, translating Ming: He thinks the learning process counts for $75 \%$ and the other part the final result occupies $25 \%$.

BS: Has it always been like this? How long has process been assessed in this way? 
Interpreter, translating Ming: All the time. He thinks that the communication between the students and the teacher during the process is very, very important. Because you can know the students very well during your communication.

The assertions discussed above may be interpreted in two ways: first, Ming may have been telling me what he thought I wanted to hear with regard to the percentages attributed to assessing process; and second, the reference to communication was perhaps key to issues experienced by the foreign teachers. We could not communicate with these students in their mother tongue, and as outsiders we were unfamiliar with their ways. Arguably, when confronted by foreign teachers' ideas about design processes, students responded by seeking clarity, and the way for them to do that was to ask for steps. It was difficult to know whether the students subsequently reflected on their learning, because we could not converse freely with them. However, at the conclusion of Ming's interview in particular I was left with the distinct impression that the influence of the foreign teaching at HNCU had permeated the thinking about teaching and learning there, particularly with regard to teaching design thinking process. Therefore, it was very difficult to truly understand the dynamics of the situation:

Reflection: At the conclusion of this interview I felt quite dispirited, because it felt like I only scratched the surface with some of the replies.

Ming was my sixth teacher participant interview and I was worried that my questions were not being understood well by Hua the interpreter, or that she was editing the responses. Although an examination of how the New Zealand teachers were working with the students within this transnational context should have been expected, much of what we had been doing at the university was presented back to me. For example, I was especially surprised to hear 'point, line, shape' expressed in one of the interviews, because I had developed a drawing and principles of design curriculum which used these building blocks of design in 2013, and these interviews took place five years later in 2018. I was hoping to hear points of view that were particular Chinese points of view rather than my points of view permeating the interviews. In retrospect, two activity systems, that of the New Zealand teachers, and the Chinese teachers were coming together, and so a hybridity of practice was forming in which object-oriented motives were potentially aligning to similar outcomes in order to relieve a perceived student creative design process problem at $\mathrm{HNCU}$. 
Shi's explanation was more convoluted as she attempted to describe in order of importance the most important aspects of the students' creative design process. In the end she admitted that the most important part was completion. However, there were no overt descriptions which would lead one to consider that there was a focus on one iteration of a design per se; rather, Shi adopted a philosophical stance towards the pursuit of perfection in the way she questioned the subjectivity of perfection:

Interpreter, translating Shi: From the perspective of the shape the layout and the style and the contents of the students works, she can judge whether the student is creative or not.

BS: How do you judge that, what is the criteria you use?

Shi [in English]: I divide into four parts, composition I think its most important, second is creative.

BS: Like the concept, the idea?

Shi [in English]: Yes, and three is vision, and four is detail.

Interpreter, translating Shi: Just now she has mentioned the four aspects, but the most important part is completion. If the students finish designing the works, that is good. But if the work is incomplete, that's not good. Sometimes that's contradictory again. Just like the wings of Venus, the sculpture with wings.

BS: Oh yes, the Winged Victory of Samothrace. [referred to as Nike]

Shi [in English]: Yes, how to judge, that is the question again.

Further questioning around Shi's answer revealed that the criteria Shi used to judge creativity appeared hierarchical. For her, in descending order, completion was the most important, followed by the visual style/look of the work, composition of the work and then detail. This criterion of detail could potentially link to creativity aptitude testing in China as was revealed in the literature as the addition of detail was an indicator of elaboration within the testing systems (Torrance, 1972).

Interpreter, translating Shi: She gives an example. If she asks the students to draw a cat, and some shouldn't just draw a sketch or the shape of the cat, but other students can give us some details including the eyes with bright, bright eyes or some fur in the details. If the students can do that she will give a higher mark than others. 
The mention of detail was something we New Zealand teachers grappled with. As I was conducting these interviews during the second delivery of the branding course, my colleague expressed surprise when she was trying to explain 'less is more' in regard to logo design. When she asked the students to keep it simple the class were unconvinced:

New Zealand colleague in conversation: They think if they keep it simple, it will not show their intelligence.

We reflected on this, surmising that this might account for differences in aesthetic styles and tastes. The inclusion of detail was exemplified by a finished student illustration that Shi proudly showed me (see Figure 8.7), whilst we had been discussing imitation and imagination.

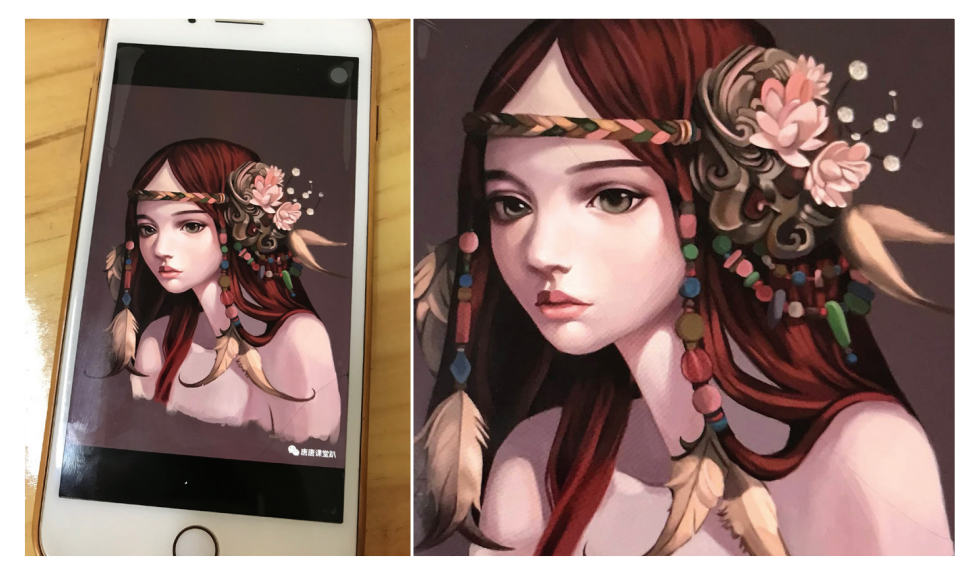

Figure 8.7. Completed student illustration (author images).

In the example she showed me it was clear to me that a creative design process was not followed, but rather the work was a copy of an illustration:

Shi: [in English]: This is a very famous illustration master in China.

BS: Famous master? Did they copy that drawing?

Shi: [in English]: Yeah.

Interpreter, translating Shi: She is eager to let the students know how to draw, and how to get the drawing techniques from the famous works.

BS: What part of the illustration are you most happy with? 
Shi [in English]: The skin. The skin is very like human skin, and second is this flower, it has more detail. It is very beautiful.

Another of the participants, Ang, contradicted both Shi and the students who asserted that their intelligence and hard work would be questioned if they did not add enough detail to their designs:

Interpreter, translating Ang: The logo should be simple because the function of the logo is to convey information, you only have to convey the information to the target audience, and if the logo is very complicated it's not very easy to recognise, its unrecognisable.

It should be noted that the excerpt above is an example of my point of view being reiterated as discussed in my reflection above. We had been discussing the difficulties for students when it came to simplicity. The excerpt which follows privileges a student I talk about, but this was done in the hope of an authentic response being revealed; however, my strategy did not result in an answer that enlightened any further understanding:

BS: The design work that I'm currently doing with the students, is around designing logos, and I'm trying to help them understand the idea of simplicity, so the communication for the target audience becomes simplified. But this morning, a student said to me, but the Chinese teachers tell me if I put more detail in, I'm working harder. So I think it's difficult for the students, because I'm saying be simple, take away, remove, and the Chinese teachers are saying add more it will show that you are working hard. I just like you to share that observation with Ang to find out if she has any thoughts about it.

Interpreter, translating Ang: So she thinks it is better to be simple.

Few teachers directly acknowledged that product over process was important. Ting alluded to the completion being the first consideration, but there was a preference for a concept or function to be interwoven with the end result:

Interpreter, translating Ting: She thinks if the students can finish the work successfully that is the best, and the second is if the students have the good idea, the good concept that is best. 
However, if this could not be achieved by the student, Zhu conceded that perfection in the guise of beauty was acceptable. This points to a form of graphic (visual) design not placing emphasis on communication design. In this instance, 2-dimensional space is arranged in such a way whereby hierarchy and stylistic or decorative treatments become the most important features:

Interpreter, translating Zhu: In his opinion, if we can combine the function and beauty together that is perfect. But if we cannot, based on the function we better pay attention to the beauty.

\section{Quantity over quality}

A concern with quantity over quality issues was found to be intertwined with the theme encouraging cultural and social rules of perfection. At the time of the classroom observations I asked Lei how she went about assessing the natural gas logo designs and the Bauhaus poster work that I had observed in her classroom. Her response was that if the students completed four logos and four posters they would receive an $80 \%$ grade, and if the students completed five, six or seven designs they would receive a $90 \%$ grade. It was her expectation that none of the students would fail:

Lei [in English]: I hope the students will be perfect, so don't go below 70\%.

BS: So what does she do about the students who have given up?

Interpreter, translating Lei: If the lower students can finish their task, even if they are not motivated, even they are not very interested, so she will get them 60 score.

BS: They still pass?

Interpreter, translating Lei: Yes they will pass.

BS: Even if the quality is not up to standard?

Interpreter, translating L: Yes, also it's a kind of encouragement.

This signalled quantity over quality, with a focus placed on a finished product that was not necessarily a perfect product. Therefore, it is arguable that here, perfection is not a focus, and thus not a cultural or social rule per se. Although Lei hoped all completed work would be perfect, the idea of her teacher motive (object) comes into question. 
Why was Lei compromising quality and rewarding quantity with a higher grade? Perhaps the answer lies in a notion that even though student perfection can be said to be a motive for teachers, quick technical skill learning which led to quantity over quality was also a motive. This revelation pointed to the emergence of third generation activity theory where multiple objects were potentially expanding learning. The mention of $60 \%$ being a pass grade was surprising when I first encountered it in 2013, but this is the norm at HNCU. Consequently, we New Zealand teachers had devised an assessment scale which took this 'high' pass grade into account, since 50\% was not attributed as a pass.

The evidence that students were seldom failed was supported by Ming and Shi:

Interpreter, translating Ming: If he fails a student that is to say, actually he seldom fails a student, but if a student fails, I mean, if he let a student fail in an exam, that is to say the students is really not good, he seldom fails them.

BS: Will Shi pass them or fail them?

Shi [in English]: Fail

BS: Does that happen very often? Do you fail many students?

Interpreter, translating Shi: Not often. Yeah because she always urges the students to do something according to the student's ability and improve.

\section{Competitions}

Shi alluded to the role of competitions (norms) in teaching practices in Chinese classrooms when she showed me the student design work on her phone, discussed earlier:

Shi: I encourage them to enter competitions.

Likewise, Zhu turned to competitions as a way to stimulate and engage the students when interest in learning seemed to lag. I had asked what some of the reasons might be that would account for students failing and what he has done about this. He suggested this situation seldom existed because most of the students would follow his steps, and 
he is very strict with the students and never moves on to the next step until they have completed the previous step. This in itself pointed to a strict linear path of learning. Zhu also said he lets students bid for the projects and all kinds of competitions. He would search the internet for suitable competitions on behalf of the students, and these would form the basis of assignments:

Interpreter, translating Zhu: This can arouse the students' interest ... Except only one or two students have some psychological problems and they will give up, but other students are good and willing to cooperate with the teachers and always finish the works on time.

BS: How successful are the students with the competitions, do they win? Interpreter, translating Zhu: Every time the students can get a prize ... if the student is successful, he can also pay the money to the students. From the website, some competitions bonus is very high.

BS: Bonus?

Interpreter, translating Zhu: Yeah the bonus, the bonus can reach to 20,000 yuan for the first prize, the lowest the third prize can get 1,000 yuan. This can arouse the student's interest, to enter the competition. They can combine the study with earning the money, that's very good ... and the students show great interest in this about the future work.

By their very nature of competitiveness, the strategy of using competitions within the curriculum is motivated by a desire to win or achieve perfection. The large amounts of prize money adds further motivation by way of reward. But the middle level activity system revealed here points to the tensions students encounter when they are confronted with a requirement to use their imagination for the desired goal, which is to win a competition.

\subsection{Theme 3: Balancing teaching practice with learning success in the division of labour}

Balancing teaching roles with learning success, the third theme to emerge from the transcripts, was quite problematic for Chinese teachers. Balancing large class sizes with student motivation was unquestionably difficult, but I believe there are contradictions in the way teachers responded to this issue. Chinese teaching styles at 
HNCU were found to range from facilitator to authority, and it was discovered that within this, a form of delegation was in place (Biesta, 2012).

\title{
Facilitator
}

Zhu exemplified a facilitator's teaching style by describing the way he divided large classes of up to 50 students into three groups to manage teaching and learning. He characterised the first and smallest group of students as those who were the most active, showed the most amount of initiative, and asked the most questions. The second and largest group was distinguished by students who were not interested in communicating with the teacher, did not care if their work was good or bad, and they were focussed only on completion and getting a grade. When I asked how large this group was, he replied that it was most of the students. The third group was delineated by 'laziness':

\begin{abstract}
Interpreter, translating Zhu: They are very lazy, they don't want to do the project the teacher assigned. So as a teacher, he thinks the function of the teacher is only to lead. The teacher plays a leading part to guide the students to do something, but not to tell them everything that they should do. To guide them through the learning process, they all need encouragement. When you encourage them they will feel better and do something good, or more active.
\end{abstract}

Zhu's promotion of encouragement points to a secondary artefact as did teaching students how to learn, or promoting self-directed learning. This was a shared desired outcome for both Ting and Yin:

Interpreter, translating Ting: In the class, she can teach the students the methods, the ways more the knowledge. There is a saying in China . . . the first one yú is the fish, and the second yú is the verb to fish, right. That is to say we had better teach the students the methods and not more than the knowledge. If we train the students' ability and methods they can do everything successfully in the future.

BS: What is the best way for Yin's students to learn?

Interpreter, translating Yin: The best for students to learn is to how to selfstudy. Because they are learning design, so it's best to let the students get their own way of study, and to get their own methods to design. And, the third is cultivate their interest for themselves. 
However, these two opinions were directly contradicted by Zhu who described an authority style of teaching where the 'system' had contributed to making the students passive, and as a result teachers had to do too much for the students.

\section{Authority}

I had asked a question about any difficulties or challenges Zhu had to face when he was preparing for his classes. He replied that the great challenge is the system and that in truth, teaching only occupies $20 \%$ of his time, and that a significant part of his time was devoted to preparation because students were not allowed to leave the classroom during class hours. He was frustrated because students were not allowed to experience actual life:

BS: What do you mean by actual life?

Interpreter, translating Zhu: Like, do some research, because to be the major in the designing, they need to look more, see more. But our system is different, it limits the student in the class.

BS: Can you tell me more about that? What aspects of the system limits the students?

Interpreter, translating Zhu: So, in the past they can let the students go outside to do more social experience, to look around. But in recent years, our school forbid to do that. Or maybe, just limit not forbidden, but limit. So, the students are forced to stay in the classroom, and the major teaching method is input the knowledge to the students, that is not so good.

Zhu's frank assertion was important because his longer experience in teaching design recognised the importance of social and cultural experience as a major contributor to solving ambiguous design problems. As his students were confined to the classroom, presumably Ting's and Yin's students were also confined to the classroom. Therefore, by attempting to teach self-directed learning and letting the students get their own way to study, the internet is the only tool available in the classroom for research.

An example of inputting knowledge was evidenced when Zhu described his preparation for a logo design class. His approach was to analyse many different logos to find commonalities for the students. The end of the following excerpt takes us in a full circle back to the beginning of this chapter, as the contentious topic of enabling 
transformation of student creativity away from imitation is repeated, which in turn begs the question, how much of an authority style of teaching contributes to students imitating work, rather than a fostering of imagination to mediate new work?

Interpreter, translating Zhu: Because in this logo designing he has found some famous logo, have some common things. For example if they [the students] design some logo for the enterprise, for the company, or the corporation, they usually use a square or a circle etc. But if they design some logos for some activity, some show, they will use the different shapes, according to the different sights. They will give the different logo designing. For the activities and the show, they will use the different shapes, and it can be various choices for them to choose. But for some companies, some special square and circles.

BS: Does he do the analysis of those logos? He is talking about an analysis, the company uses a square, or different shapes, does he do the analysis of those logos himself, or do the students do that?

Interpreter: According to himself.

BS: So he has done the analysis?

Interpreter, translating Zhu: Yes, the seeing and he can judge. Maybe they have some different variation, different change, but the big shape or the big...

BS: Kind of like the trend . . I know what you are saying, yes. So, when it comes to teaching the students, what does he think the benefits are of imitating those existing logos. How do the students learn from that? How do the students learn from imitating?

Interpreter, translating Zhu: Through the imitation the students will know what most logo designs have in common, in shape, and second they will know the colour, the students can know how to apply the different colour. They will know the swatch [colour], and then the third, the students can know the stories background, the background story of the designing. From all this, the students can know something and get some inspiration.

Zhu highlighted that as a teacher he played a major role in leading the students. This is quite different to how I teach. Students are encouraged to conduct their own analysis, and through this decide for themselves what is useful or not. The question raised here: are the Chinese teachers doing too much for the Chinese students within the division of labour? When I asked Lei if the students would have to design their own natural gas logos (discussed at the beginning of this chapter), she told me she had found the logos 
herself on the internet for the students to copy in order to save their eyes, in other words, to save them the time, owing to expectations that the work would be completed before the next day. At the time I had thought it was kind of her. But this recurring pattern continually suggested that Chinese teachers do too much for their students, to the detriment of fostering curiosity and discovery. Therefore the development of critical thinking skills is potentially compromised.

The other consequence of the teachers doing too much for their students was reflected in the many assertions of 'laziness' in general resulting in lack of motivation (engagement with the object). Shi mentioned it at the beginning of her interview (discussed previously). Lei attributed laziness to copying, Yin blamed a bad attitude, and Dong asserted that despite their laziness, students completed his assignments:

Interpreter, translating Lei: The bad aspects [of copying is] that the students are becoming lazy and they don't want to be creative. They don't want to think about their own ideas.

Interpreter, translating Yin: It's all about attitudes the learning attitudes. For example, if the student is always absent, and they, the students that are absent or who are very lazy in the class, or they some of them do some other things instead of designing.

Interpreter, translating Dong: Even though they are lazy, but they still finish their work, but not very how do you say, not willingly.

The comments made by the Chinese teachers regarding 'laziness' are consistent with what foreign teachers encountered in the classroom and one of the case study participants referred to himself as lazy. However, 'laziness' can account for a multitude of barriers that arise from an individual's context or situation including tiredness, boredom, procrastination, anxieties or fears about not being good enough, or external technological distractions (gaming, social media, and online shopping) that were witnessed.

\section{Delegator}

To ensure that the students did complete the assignments on time, Lei adopted a strategy whereby she enlisted help from the top students: 
Interpreter, translating Lei: Her method is to force the students to finish the project on time. Teachers should be very strict with them. The usual practice for her is to let the top students do the project first, with high quality which can influence the other students to follow.

BS: Do those students help the other students?

Interpreter, translating Lei: You know the top students can do it by themselves and the middle level students can get help from the top students and from the teachers. While for the lower students, some of them just give up, so the teacher can only offer help when they are interested.

By focussing on the top students first, the division of labour is shared because the top few students help the teacher, which is potentially a good strategy. However, this precludes other potentially talented students from contributing to this role, in other words, the average students. Passing students at the bottom level through minimal course learning potentially results in students becoming demotivated, and thus restricts the possibility of success in the long term. Equally unconvincing is the assertion that passing low level students encourages student engagement. Arguably, if the students were not interested to begin with, they are unlikely to fully participate in the course learning, and yet minimal effort is rewarded.

\subsection{Conclusion}

When I began the analysis of the teachers' transcripts I worked on the idea of a tension intersection whereby the teachers as part of the student community were impacting or influencing student production. This was depicted in figure 8.1 at the beginning of this chapter. However, as the analysis progressed, it became apparent that the three themes that emerged from the teacher interviews needed to be depicted as a separate activity system within a third generation model, and so I present Figure 8.8 for the following discussion. 
Top level tensions found between subject, object and three-level hierarchy of artefacts.

1. What do teachers think about the learners? 2. What does this mean for the teachers?

3. What learning strategies are used?

Secondary Discursive constructs (1. Lazy. 2. Frustrated. 3. Promote competitions to arouse learner interest, to win. Promote encouragement and self-directed learning)

Tertiary Process (3. Reassemblage practice - spectrum of acceptance with copying and imitation, learning steps)

Tertiary Abstractions (1. Unimaginative. 2. Challenging to encourage imagination, lack confidence to enable learner creativity transformation)

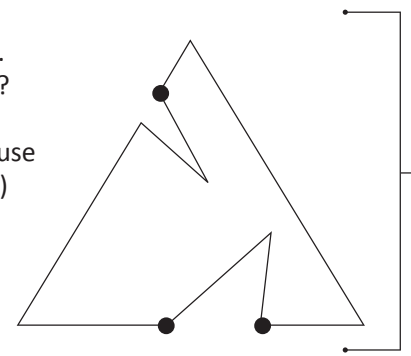

Mediating artefacts

Theme (1): Managing the effects of primary, secondary and tertiary artefact tensions

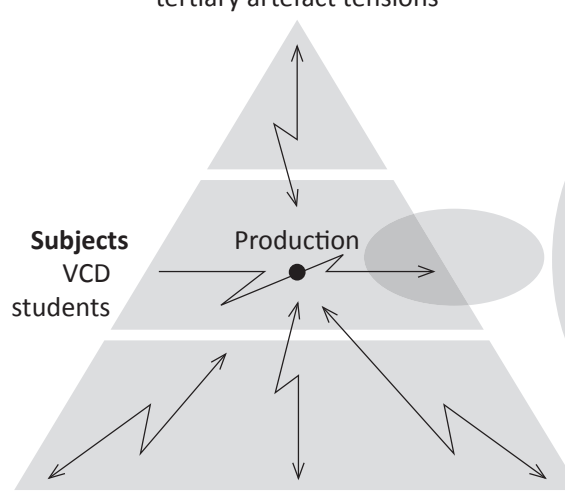

Rules

Theme (3):

Navigating local teachers and

and imported classmates

learning practices at HNCU
Object $_{3}$

Linear product over process
Mediating artefacts

Theme (1):

Reconciling imitation

with imagination

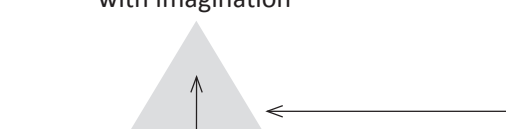

bjects 2

perfection

aesthetic

/style,

detail

Subjects

Chinese

teachers

Object 3

Iterative

(formulaic)

process

Theme (2):

for ideation

Adjusting to collaborative learning approaches, (2A) Responding to teacher guidance

$\begin{array}{lr}\begin{array}{c}\text { Rules } \\ \text { Theme (3): }\end{array} & \text { Community } \\ \text { Encouraging } & \text { Theme (2): } \\ \text { cultural and } & \text { Balancing teaching } \\ \text { social rules } & \text { practices with } \\ \text { of perfection } & \text { learning success }\end{array}$

Middle level tension found between two conflicting activity system nodes. Theme (3).

(1) Tertiary artefact (imagination/transformation from imitation to creating) and rules (competitions)

Base level tension found where an introduced cultural activity object (competitions) as a strategy to trigger a developmental learning phase. Theme (3)

Figure 8.8. Analysis of teachers' interviews/data.

The three themes that emerged from the teachers' analysis echoed the themes found in the first two case studies, and three AS nodes were implicated.

1. Mediating artefacts: Reconciling imitation with imagination (tertiary artefact);

2. Division of Labour: Balancing teaching practices with learning success.

3. Rules: Encouraging cultural and social rules of perfection. 
The first theme evolved to focus specifically on tertiary artefacts (processes from the mind). The second theme to emerge was more general, as it sought to understand what the constraints and difficulties are for teachers and how this manifests for student learning. The third theme examined how cultural and social rules of perfection are encouraged and enacted by the Chinese teachers.

Reconciling imitation with imagination was located as a top level activity system tension and it highlighted a pragmatic approach used by the teachers when it came to process. It was found that in the opinions of the teachers overall, most recognised an iterative process as an important aspect of learning design processes, yet found it challenging. Teachers asserted the students were lazy and unimaginative, yet they themselves lacked ways to address the gap between teaching applied skills and implementing creative innovative thinking. To encourage student engagement the teachers sought a formulaic process for ideation which in itself is paradoxical as arguably iterative processes are not linear or formulaic, they are more likely messy and tangled. Zhu mentioned learning steps which are strictly followed. Others use assemblage practice, and contained within this, there is a spectrum of acceptance when it comes to copying and imitation. This was seen in the examples Shi showed me on her phone, and the work Lei sent me when her environmental project highlighting the negative effects of smog pollution was completed. The issue of copying and imitation is inherently problematic as teachers claimed they disliked previous learning undertaken inside training schools at high school level; however, to varying degrees, this was a part of daily teaching practices. The real complexity for students is in ascertaining to what extent along the spectrum of acceptance is copying acceptable. And, with different teachers' perspectives, the transference of the practice between local and foreign classes is difficult for the students.

Within the division of labour, balancing teaching roles with learning success has been most revealing. Arguably, the interviews revealed aspirational stances, but my observations indicated that much of what the teachers were saying was true. Balancing large class sizes with a range of student motivations was unquestionably difficult and something I could relate to having experienced it at the university. The prevailing practice of passing students who are working at very low levels in the hope that they 
become encouraged is fraught because minimal effort is rewarded. In an attempt to address the issue of 'laziness' which frequently permeated the interviews, teaching styles ranged from facilitator, to authority, to delegator. Unexpected was the extent to which Chinese teachers will go in order to help students. Despite best intentions, the apparent authoritative teaching style where teachers input knowledge limits creative opportunities. Critical thinking within creative design processes begins in the research and preparation phases of a project. Doing this work for students potentially restricts the overall holistic design process as it fails to ignite curiosity and the thrill of discovery.

Cultural and social rules relating to the question of perfection were examined. The difficulty for the research was to tease apart what the teachers were saying about process, and how these motives were viewed. The mystery of how to teach western creative design process aspects appeared to conflict with Chinese cultural preferences and motives. Completion of high quality design work was found to be the most important motive for teachers, and this was widely underpinned by motives focussed on perfection, details in the work and aesthetics or style. Aesthetics or style was often mentioned, and although teachers recognised and would have preferred the work to be led by a concept, more often than not greater emphasis was placed on stylistic or decorative treatments. Subjectivity became part of the question, for example Shi's reference to the Winged Victory of Samothrace (Nike), a Greek sculpture with no arms or head, considered to be the epitome of beauty and perfection, yet the sculpture is damaged, and thus flawed. Working against the notion of high quality completed work was evidence of quantity over quality practices, for example, in Lei's classroom, accuracy (quality) was less important than quantity more completed work led to a higher grade.

The discussion chapter, which follows, combines the three analysis chapters for an indepth discussion that draws on the literature. Underpinning the discussion is richness of understanding about Confucian educational thought existing within the social and political landscape in China. Learning practices both familiar and unfamiliar to students, including previous models encountered by them in the classroom together with adjustment to new practices, were seen to directly impact student actions. Most 
pronounced were imported educational practices which resulted in tensions and contradictions between linear and iterative processes, collaboration within the division of labour and copying and imitation perspectives. Non-creative and creative activity outcome conclusions are drawn, and a fresh perspective emerges; this is discussed in the following chapter. 


\section{Chapter 9. Discussion}

In this chapter I discuss key findings from the case studies and teachers' interviews presented in chapters 6 to 8 in relation to the literature and from a cultural historic activity theory (CHAT) perspective. As the discussion proceeds, interrelationships that were found to exist between the visual communication design (VCD) students, the New Zealand teachers, Chinese teachers and the contradictory cultural motives and contexts that were encountered are elaborated. Within this context, prior learning practices influenced student actions and group activities. Previous educational practices and ways of doing things in the design classroom resulted in a misalignment of New Zealand teacher and Chinese student motives. Within their conventional courses the students were confident and capable, but when it came to the transnational courses, students had to contend with linguistic and cultural challenges, and were asked to set aside what had worked for them before design learning (Hilton, 2016). The tension created by this misalignment created opportunities for changes to be made to practice and outlook; however, it caused a certain amount of disorientation, confusion and frustration for the students in the classroom. They, and possibly their teachers, were asked to take on board or consider design thinking processes, and much of the data captured the uncertainty, the dissonance, and the fear of getting it wrong when letting go of the familiar. Yet, I argue that emerging from this, it was discovered that VCD learning at Hunan City University (HNCU) uses creativity as craft in a way that is unique to this particular social and cultural context (Glăveanu, 2018). I came to recognise how Chinese teachers, past and present, drew from sociocultural traditions and the wider context central to the creative process to encourage and promote creative learning. Within this the relevance and acceptance of imitation and repetition, and use of precedents or models and mentor support was critical for the development of skill. Over time, this use and practice may lead to mastery. But rather than using this finding to make an argument based on comparative judgements which privilege western points of view or ways of doing, I argue this finding enables significant insight into a local cultural practice that has validity in its own right and which cannot be reduced to plagiarism and/or lack of creativity (Glăveanu, 2018). Therefore, the research contributes a nuanced and complex understanding of creative and non-creative actions 
and activities that takes into account cultural, historic and social practices of this particular learning community.

As my understanding of CHAT deepened, an appreciation of the affordances and opportunities of activity system (AS) analysis increased. The combination of research strategies used in this study enabled an identification and understanding of complex cultural historic activities and social interactions of creative design learning at HNCU. I grappled with activity theory as I tried to tease out differences between artefact or tool-mediated activity and context and how these were articulated, or how distinctions between action and activities were made. Illumination moments included: Wartofsky's (1979) way of thinking about artefacts and tools not as separate entities but rather used in combination, hence the notion of "artefact as tool" to extend or mediate the aquisition and transmission of learning for production and reproduction (p. 201); Engeström's (1993) articulations about contexts from an activity system point of view whereby “contexts are activity systems" (p. 67); and the way Leont'ev $(1981,2005)$ distinguished between action and activities by attributing action to an individual and activities to a group which, in turn, connects the division of labour to an object.

A three-level hierarchy of the artefacts model (primary, secondary and tertiary artefacts) in which I drew from Wartofsky (1979), and Cole (1999), was developed for the analysis. This taxonomy not only extends CHAT for visual creative scenarios, it also enabled insight into the way object-oriented motive/s drove interactions between the subject-artefact-object relationships at the top level of the participant dyadic activity systems (Foot, 2014). Prior to beginning my analysis, I was unsure of Foot's assertions regarding the constant tensions and contradictions being exposed by this particular (subject-artefact-object) relational aspect. A large number of secondary discursive constructs which reflected behavioral and emotional artefacts, for example tiredness, appeared to be blocking actions and activities which contradicted the notion of expanded sequences and cycles of learning. However, owing to the way in which the students were focused on goals of completion, an ability to overcome these object tensions was evidenced by the way the students accelerated their enthusiasm towards the end of the projects. 
Leont'ev's conceptualisation of a three-level model of activity theory in which objectoriented motives drive the top level, action driven by goal(s) operates the middle level, and conditions and tools in action drive the bottom level, was extremely useful for the analysis (Engeström \& Miettinen, 1999). The notion that the top, middle and base levels of Leont'ev's model was contingent on overlapping layers moving up, down and through the activity system, and the 'misfit' between these activity system layers and core components such as rules revealed tensions and contradictions which were evidenced as learning action(s) (Foot, 2014). An example of this was evidenced in the Miao project when Connor drew on his past and present learning experiences in an attempt to consolidate a new practice through the use of calligraphy. The analysis also drew on the differences and interpretations about object between Leont'ev who was concerned with individual activities and Engeström who extended CHAT to include collective and cultural contexts (Kaptelinin, 2005). This distinction between objects has resulted is an elaboration of third generation CHAT which presents the overall research findings for this discussion.

For the purposes of the following discussion which addresses the research questions three sections are presented, beginning with the sub-questions, detailed next.

\section{Main research question:}

How do VCD students at a university in China engage with creative design process learning?

Two sub-questions:

1. How do previous and current Chinese teaching and learning practices shape VCD creative design process learning?

2. How do imported and unfamiliar educational practices influence VCD learning activities?

Interwoven through each section are perspectives from the literature in combination with findings from the two case studies, the branding and Miao cultural projects, and the teachers' interviews. The case studies were focused on the students, and the 
teachers' interviews were conducted to uncover teaching methods within the cultural classroom context.

\subsection{How do previous and current Chinese teaching and learning practices shape VCD creative design process learning?}

A key focus of this $\mathrm{PhD}$ research was to understand how previous educational practices had influenced creative design activities of the VCD students at HNCU. Furthermore, this first sub-question was devised in response to Engeström's (2001) third AT principle "historicity" in which problems may be understood in relation to their history (pp. 136-137).

There were two object-oriented motives that were found to be consistent across both of the case studies from the combined activity systems of all participants. The first, evidenced students focussing on the product or goal rather than using process (product over process), and the second revealed motives for students' concerns related to producing excellence or perfection. Consistencies continued when the teachers' interviews revealed object-oriented motives that related to completion of high quality work, and perfection (again product over process). It can be argued that these objectoriented motives were shaped by previous and current pedagogical practices, and that Confucian cultural values underpin these educational practices.

\section{Object-orientated motives in the production of product over process}

The students' previous educational experiences and practices contributed to their focussing on the goal of completion (product), rather than adopting a more organic open-ended process. I first identified this in the branding project when Amy and John were fearful of jumping out of their initial circle concept. When I challenged their progress in the classroom there was a resistance to move away from this concept. Amy would have preferred to continue with her first concept (the circle), as she believed further experimentation resulted in lack of focus. This fear of deviating from a single concept and attempts to follow a step by step or linear pathway resonates with previous research. Yuan et al. (2018) found that foundational students at a university in 
Changsha preferred Type A learning (from doing to seeing) which involves mastering skills from concrete experience. Yuan et al. discussed their step-by-step pedagogical process, the kind Amy was looking for, as a way to encourage Type A learning. Zhu, one of the HNCU teachers, revealed that he was very strict with his students - they were unable to move on to the next step until they had completed the previous one. The assertion made by a Northern European teacher that students lost their way because there were no steps, was reiterated by my New Zealand colleague who asserted students sought clear step-by-step direction. During the Miao project, Aaron suggested that his Chinese teachers guided his learning in a specific way; they did not allow the students to 'self-design' an idea. One student reflection stated that in Chinese classes "we have to do [it] the same way, only one way could be best" (SR18). Another student reflection compared the imported pedagogy with Chinese pedagogy by writing "this class has the biggest different focus on divergent thinking and is not constrained by directional thinking or textbook knowledge" (SR37). In my experience there were constant requests for textbooks to be supplied to the students, and the staff became frustrated with me when I could not recommend any 'good' textbooks. I explained that we did not use textbooks owing to an educational 'studio-based' approach which allows for creative design learning to shape and guide the teaching through "reciprocal reflection-in-action" on a daily basis (Schön, 1987, p. 163). But, perhaps the bigger story of this finding was the way in which motives of product over process were intrinsically linked to motives of excellence or perfection, which can be traced to historic and familiar or current ways of teaching and learning at HNCU.

\section{Object-orientated motives in the production of excellence or perfection and encouraging cultural and social rules of perfection}

A desire to produce an excellent or perfect solution (something being faultless through actions or process of improvement) was a recurring motive found throughout the dyad and teacher interviews. In the branding case study, Lily wished she had started in a more perfect way to ensure a perfect outcome, and in the teachers' interviews, although many advocated design processes as a way to guide open-ended possibilities for creative solutions, achieving perfection was seen as the ideal outcome. Examples of this were highlighted when Shi proudly discussed a completed student illustration 
which had reproduced human skin and a beautiful flower, and when Zhu conceded that if students had difficulty in combining a perfect concept function with beauty then creating something beautiful was the preferred outcome. This privileged a form of graphic (visual) design which did not place emphasis on communication design, but rather 2-dimensional space was arranged in such a way that hierarchy and stylistic or decorative treatments became the most important features. However, this way of thinking about perfection in relation to a thing, or a design outcome can be challenged when viewed in the context of differing aesthetic preferences and Confucian cultural values about perfection. Cultural connotations of perfection with its purpose of attaining the highest level of morality in learning from Confucianism identifies that both meanings of perfection are so intertwined that new conclusions may be drawn.

There were two ways this was evidenced. First, during the Miao project, Aaron's father and a teacher arranged for Aaron to enter ten design competitions. This example is in keeping with literature suggesting students have to compete in various competitions to become recognised (Jiang et al., 2018). It can also be understood through the cultural custom of rite $l i$ (Chun, 2018). To recap, rite $l i$ encompasses social interactions of humans, nature and material objects (the context) within progressive codes of hierarchy and morality (Chun, 2018; Hung, 2016). Aaron's actions and his discussion of the role of his parents in his education clearly signalled his acceptance of their guidance for him. He did not question the demands that were placed on him, because he aspired to reach a higher level of morality, good or perfection, for the family group. When Aaron won a competition, the cultural meaning of perfection became intertwined with perfection of a solution. In other words, his competition entry was deemed the most perfect, and so his parents and teachers were satisfied. Through the lens of activity theory, each party played a role in the success, and the activities and motives of all participants became aligned (Leontyev, 1981). However, it was difficult for Aaron to simultaneously manage family expectations and his course project work. In class, tensions arose in the dyadic division of labour. Time ran out for Aaron and so he and his partner Rebecca were forced to complete the 'collaborative' components of the design thinking phases on their own. 
The second way the cultural meaning of perfection became intertwined with perfection of a solution was evidenced during my visit to the high school training school in Changsha. For nine months the Changsha high school students reproduced photographic portraits, still life paintings and practised gestural drawing often until late into the night. Although one cannot suggest that all creativity orientated students in China would be required to participate in this mode of training, three of the teachers at HNCU (Shi, Ang and Zhu) identified that all the students who participated in this study had been to such schools. The predominant focus of learning at this training school was to create a perfect solution, and the teachers encouraged continual, and slow, repetitive processes. Therefore, the criticisms about this type of learning leading to negative outcomes for future design learning are perhaps justified (Niu \& Sternberg, 2006). There were also similar forces in play between the training school students and their parents as there were with Aaron and his parents when it came to winning competitions. At the training school success for all parties would be admittance to a university, and this cultural meaning of perfection followed the ideals of moral behaviour and goodness.

The role of competitions in learning highlighted base level AS contradictions which emerged as significant insights. Aaron's participation in competitions along with the roles played by his parents and Chinese teachers were guided by rules of the VCD discipline itself. To explain, the actions of Aaron, his parents and his teachers involved evaluation of many amateur and professional design opportunities to ensure Aaron would be successful in achieving his career aspirations within the VCD discipline. Object-oriented motives drove the group (Aaron, his parents and teachers) attempts to consolidate a new practice (entering competitions) that extended Aaron's abilities beyond the boundaries of his classes and the university. In doing this, the group drew upon complex sociocultural interactions and relationships, and prior experiences relating to failure and/or success. This acknowledges Engeström's 'collective' interpretation of object which also includes design judges who potentially come from the design profession (Engeström \& Miettinen, 1999). Thus, the object of activity within this expression of third-generation CHAT was defined by the problem space which contained three facets. First, the object that was acted upon was the concept for the competition, second, the best way to represent a concept was located, and third, a 
desired outcome was reached when the concept won a competition and so all parties were successful, and the way was paved towards the design profession (Foot, 2014). The wider use of competitions in pedagogy at HNCU opens up this scenario for all VCD students at the university.

The theme encouraging cultural and social rules of perfection that was encountered during the teachers' interviews underpins the interpretations just discussed. The theme also provided answers for the sub-question, how have previous and current Chinese teaching and learning practices shaped VCD creative design process learning? It can be argued that student object-oriented motives in the production of product over process, excellence and perfection are derived from historic and current trajectories of learning where goals of high quality completion through guided and linear pathways were and still are the norm at HNCU. However, the way in which these educational practices can be interpreted in a positive light relates to conclusions drawn from the next research questions which follows.

\subsection{How do imported and unfamiliar educational practices influence VCD learning activities?}

This sub-question aimed to address a number of issues. The first is that of stereotypes and misunderstandings of Chinese student engagement with creative design process learning activities that were designed for the students at HNCU (Niu, 2007, 2012; Yi et al., 2013). The second, concerned the view that western ontological constructions of creativity and creative design processes, and their associations with freedom and liberal democracies, would be challenging in a Chinese context (Keane, 2006). Associated with this has been the situation where my approaches to teaching design thinking have left little room for other learning approaches such as imitation, repetition, and linear pathways. In consideration of these issues, I turned to Engeström's (2001) activity theory principle of multi-voicedness which highlighted the importance of carefully interpreting multiple participant voices in order for new perspectives to emerge. 
Students who progressed to an undergraduate level of VCD education at HNCU had successfully traversed prior Chinese educational practices such as the training school mentioned earlier. They chose to participate in an international programme that was offered by the university. Within this context, the students were challenged by the imported and unfamiliar design learning practices and they were expected to set aside what had worked for them before in their previous art and design studies. The navigating local and imported learning theme arose from the tensions associated with students having to let go of the familiar, and dissonance and uncertainty were voiced. In particular there were differences in the way Chinese and New Zealand teachers addressed linear and iterative learning practices, collaborative activities within the division of labour and copying and imitation. The practice of copy and imitation was revealed to be one of the main problems found in the data, although I argue that from these contradictions (Foot's (2014) misfit), a unique craft practice arose which drew on social and cultural contexts.

\section{Linear and iterative processes}

When designing the curriculum for the branding and Miao cultural projects, my colleague and I decided to focus on the iterative ideate and prototype phases from the d.school Bootleg cards (Stanford d.school, 2018). There was little emphasis on completion per se. Our ideas of what constituted creativity and innovation were rooted in our models and ideals which revolved around the notion of originality (Cross, 2007, 2011). Our aim was to create a process-oriented learning environment where there was room for collaborative, serendipitous, magical and illuminated activities to emerge (Neumeier, 2005). We believed that these were key ingredients for opening up new creative and innovative ways of learning. Our overall intention was to introduce strategic design thinking processes and iterative processes as a way for the HNCU students to create new work.

Design thinking was an imported process introduced into the classroom, and the students grappled with unfamiliar ways of working. An example of this was found in the branding project, when Lily was confronted by what she termed as different testing systems. Lily grappled with conflicting New Zealand and Chinese teachers’ 
expectations and processes. When her rough sketches were praised by the New Zealand teachers, and deemed original in their execution, questions were raised about what constituted good work. Implicit within this were concerns that different sets of rules and aesthetic tastes would be applied in the evaluation of the work. In the analysis of the branding project, three out of four of the dyads were found to be working within linear processes. However, at one point in the idea generation process, the motives of one dyad, Hana and Michael, became aligned to my motives. The way in which they descibed their shared actions to generate a logo concept revealed a glimpse of increased ideation, which was a key focus for this project. Furthermore, in the Miao cultural project the work Carl and Susan conducted clearly aligned with my motives (see p. 183), and it was delightful when Nina, one of the participants, genuinely embraced an iterative process (see p. 181). She discussed the value of thumbnail sketches in the ideation design thinking process, thus evidencing that sketching (a base level tension) triggered a developmental phase for her. Although these findings revealed motives of participant engagement with iterative process, only the activities of three participants, Hana and Michael from the branding project and Nina from the Miao cultural project, were directly associated with this finding.

Therefore, it can be argued that overall the tensions between New Zealand teacher and student motives that occurred, concerned misaligned Chinese linear and imported iterative process learning practices.

\section{Collaborative practices in the division of labour}

The revelation that it was unusual for students to engage in collaborative practice in their learning seemed paradoxical and raised questions about China's collectivist society. Findings relating to the success of the design thinking collaborative approaches, commonly used in New Zealand classrooms, became questionable in the HNCU classrooms. Mullen (2017) argues that China's education system is test centric and market driven and this may have influenced how students reacted to collaborative activities. Although the students were assessed individually for both projects, collaborative practice was encouraged and in some instances directed. I believed students were working collaboratively; however, Lily and Connor identified that the students adopted a system whereby only half of the class was actively working at a 
time. To a certain extent the dyad formations were compromised, as was highlighted by Aaron and Rebecca who ended up working individually, and Ricky who carried on working by himself when Simon fell asleep. On occasions such as these, Amy identified that she turned to her classmates as a way to move forward with her individual work which suggests Vygotsky's (1978) zone of proximal development (ZPD) was at play. Positive outcomes resulted from this approach and a middle level AS tension was encountered. Amy's learning actions were focussed on her goal, which was to find a way through the design block, and so by analysing the situation she tried implementing new models or ways of doing which drew on her classmates' activities. However, teasing apart the differences between using others' ideas and concepts (appropriation) as opposed to being inspired by others within ZPD and through bouncing ideas backwards and forwards (transformative owing to ideas being pushed along more quickly) was sometimes a little ambiguous. For example: "my partner share me her pattern" (SR15), and "when I hit a bottleneck, I went to see what other students were doing, and they had elements or patterns that gave me other ideas" (SR24).

Despite these ambiguities there were instances of genuine shared interactions. It was common for students to mentor and help each other so that no-one was left behind. From the 100 reflections gathered at the end of the Miao project, almost half (45) of the students expressed thanks to their partner or other classmates. For example: “... friends, they are working hard to help me" (SR02), and "thank you for your help and support, which made me feel very warm and happy" (SR27). In general there was a real atmosphere of care and peer support witnessed inside the classroom. Students were often observed physically working on each other's designs, and offering encouragement to their peers. At times they self-initiated class directives to help clarify the teachers' expectations. Outside the classroom, the way in which Susan and Carl described an organised dormitory culture where groups of students managed time issues on Sunday afternoons to help each other, offered real insight into a practice that was unique to this context.

However, there was a reluctance to offer any form of peer critique, and both Aaron and the interpreter confirmed that Chinese students are uncomfortable with this practice. 
Thus, a contradiction was found to exist between my expectations and the students' willingness to engage in this practice. New Zealand students have been socialised into, and have learnt how to provide peer feedback or critique, with the aim of increasing self-direction (Cook-Sather, 2014), whereas this was a new and uncomfortable process for the VCD students. This was an instance where I assumed the students had these skills as it is common in New Zealand classrooms for students to reflect on and offer peer critique within either informal situations or formal presentation settings. One possible explanation for this could relate to the concept of miànzi (face) in Chinese society (see p. 88). Or, in Aaron's opinion, it seemed that any critical exchange between students in the classroom could only become something of value once opinions had been filtered through the teacher. This highlighted Confucian educational practices where the teacher is perceived to have progressed through hierarchical structures of learning within the context of relationships and moral responsibility, and so is considered more informed (Hung, 2016). In support of this, students tended to wait for an approval from the teacher before moving on to the next design process stage. When students received some form of help within this relational aspect of the division of labour (DOL), they seemed overwhelmingly receptive, for example: "the teachers' unconventional thinking had given me endless enlightenment" (SR66) and 'the teachers' reverse thinking, open up new thinking makes me wonder how much magic can be produced by the brain" (SR78). Although the data reflect an openness towards iterative approaches, it indicates there were tensions associated with a lack of individual readiness to initiate further action within the design process without teacher support.

In answering the question, who is doing what work? within collaborative practices in the DOL, there was a strong sense that the Chinese teachers contributed a great deal to the students' learning. I found that teachers undertake a high level of preparation on behalf of the students. For example, Zhu analysed many different logos to find commonalities for the students, which arguably limits the opportunities afforded through student research. Another example was found where Lei, located posters for the students to copy in order to 'save their eyes' and to allow enough time for students to complete the learning tasks. Despite their efforts, teachers were frustrated by an apparent lack of student motivation. The teachers' pedagogical approach was complex 
owing to the levels of preparation and the widespread practice of passing all students, no matter the quality of work, as a means of encouragement. A recurring pattern of student 'laziness' underpinned the teachers' frustrations, and it was asserted student progress was limited as a result. However, the way 'laziness' was described can account for a multitude of barriers that arise from an individual's context or situation including tiredness, boredom, procrastination, anxieties or fears about not being good enough, or external technological distractions (gaming, social media, and online shopping) that were witnessed in the classes by me. It can also be argued that the demanding timetable, comprising approximately 35 hours including a physical education class to provide some relief, would challenge the most dedicated student.

Teachers used a range of styles, moving from facilitator to authority, and implemented a form of delegation to balance teaching roles with learning success, however, the result of these approaches was limited (Biesta, 2012). Zhu concluded that as the students were confined to the classroom by the system, they were not exposed to experiences that could enhance their learning and so the internet was the only research tool available. This was countered to a certain extent by the field trip to the Miao villages in Guizhou as described in the context chapter, but we New Zealand teachers had similar issues. Requests to visit the tea street in Yiyang as a group were dissuaded which to our minds was detrimental to fostering tangible curiosity and discovery about the tea experience for the branding project. Gaining experience with products is critical to designing an emotional branding process (Gobé, 2009). Ricky, one of the participants, highlighted this problem when he talked about trying to design a brand for a product with which he had little direct experience, and there were few memories that he could draw on that were relevant to the product.

\section{Copying and imitation}

There was widespread condemnation for copying and imitating practices by the Chinese teachers. The theme reconciling imitation with imagination in Chapter 8 captured the myriad of anxieties and frustrations around the practice. Seven out of eight teachers at HNCU directly discussed the issue without any prompting. Shi blamed student laziness and lack of thinking abilities at the beginning of her interview 
on copying, Yin attributed blame to a bad attitude, and Zhu imposed rules that suggested students were not allowed to use other people's ideas. Ang's explanation was most interesting when she tried to explain the tensions. She asserted that imitating was an easy shortcut to success because copying technique, such as applying paint in a particular way, was a different process to learning to think independently and for the students to use their imagination to create new ideas.

Ang's insight echoes a theory not engaged with in the literature review, but I draw on it here owing to the way in which it captures an age old problem. In 1934, Dewey asserted that "major developments in the major techniques of painting during and since the Renaissance ... were connected with efforts to solve problems that grew out of the experience itself, and not the craftsmanship of painting itself' (p.147). During the Renaissance artists became preoccupied with a problem, which was how to depict the concept of three-dimensional perspective on a two dimensional surface (Dewey, 1934). This activity connects to both Leontyev's $(1981,2009)$ and Engeström's (2001) interpretations of object. From Leontyev's point of view, the object of the painting activity (the problem of how to depict perspective) gave the painting process a determined direction. The media (paint) was not the problem; rather, the role of imagination in determining how perspective could be depicted drove the painting (the problem space). When more than one painter became involved in trying to depict perspective, Engeström's (2001) interpretations of object-orientated motives are highlighted. Thus, when a community of Renaissance artists engaged with the problem of depicting three-dimensional perspective, their culturally mediated activity systems eventually paved a way for a solution. Therefore, from the problem space a desired outcome was reached (Foot, 2014). Ang suggested that teachers had a role to play in enabling the transformation from technique imitation to that which envisages conceptual creative problem-solving, but this notion appeared problematic for some of the other teachers. Zhu was challenged when it came to teaching student creativity and Ming assumed New Zealand ways of teaching might contain the key to unlocking a magic formula in order to teach creative thinking processes.

Notwithstanding these arguments, during the branding project I discovered students at the opposite ends of the classroom drawing similar teapot logo designs plainly copied 
from the internet. Furthermore, Shi and Lei provided concrete examples of the practice when they showed me student designs that used internet sourced images or parts of images for durex, a milk drink, and the Rubin vase that had been used for a poster design. It was apparent that there was acceptance of copying and imitation at HNCU. This is discussed further in the next section.

\subsection{How do visual communication design (VCD) students at a university in China engage with creative design process learning?}

Through the branding and Miao cultural project analysis chapters I, along with my colleagues, contributed different voices and perspectives in the classroom. During the dyad interviews it became apparent that our motives and goals were different to those of the students, and so our motives (objects) were largely misaligned. Therefore, our actions and activities in the psychological tradition of Leont'ev (1981) were different. I was mindful that my imported teaching practices, which included design thinking, had the potential to create an uneasy relationship between traditional and modern design education approaches taught at HNCU (Buchanan, 2004). Ideally, student engagement with an action or activity is foreshadowed by a teacher's deep awareness of the types of motives that are in play in the classroom (Disarro, 2014). My motives for the two particular VCD pedagogical projects examined in this research were process-oriented which related to unlocking strategies, and techniques in order to transform creative design process learning. The goals or purpose related to embedding design thinking process learning, encouraging the students to use iterative processes; final outcomes per se was not the aim. However, for the students, their object-oriented motives related to productions of product over process, and excellence or perfection that has been discussed. This has the potential for conclusions to be drawn from the data which would seem to affirm vexatious questions and stereotypes about Chinese students' creativity (Ceppi, 2017; Kember, 2016; Niu, 2012; Yi, Hu, Scheithauer, \& Niu, 2013).

In consideration of Engeström's (2001) multiple viewpoints within third generation activity theory in which collaboratively constructed viewpoints may be articulated through expanded objects, the findings described in the paragraph above seemed to be offering insight into an opposing form of expansion. My problem here is that if the 
expanded object is potentially transformative and an agent for change, how should this be descibed if the opposite was found to be the result of colliding objects? For some time I toyed with the idea of contraction; however, a more concrete answer was found in Engeström. Non-expansive learning actions, which reinforce existing practices, means "processes of innovation knowledge creation are not pure [as] there are both expansive and non-expansive phases, both steps forward and digressions" (Engeström, 1999a, p. 391). In many ways this explanation from Engeström connects to Tikhomirov's (1999) theory of creativity. In the theory, comparison, reproduction, assimilation, and copying are the functions of non-creative activity (digressions), whereas construction, generation and creation of the new (steps forward) are most important operations for creative activity. Figure 9.1 presents an interpretation of the opposing student and New Zealand teacher objects found in the case study projects in a representation of a third generation activity theory minimal model (Engeström 2001).

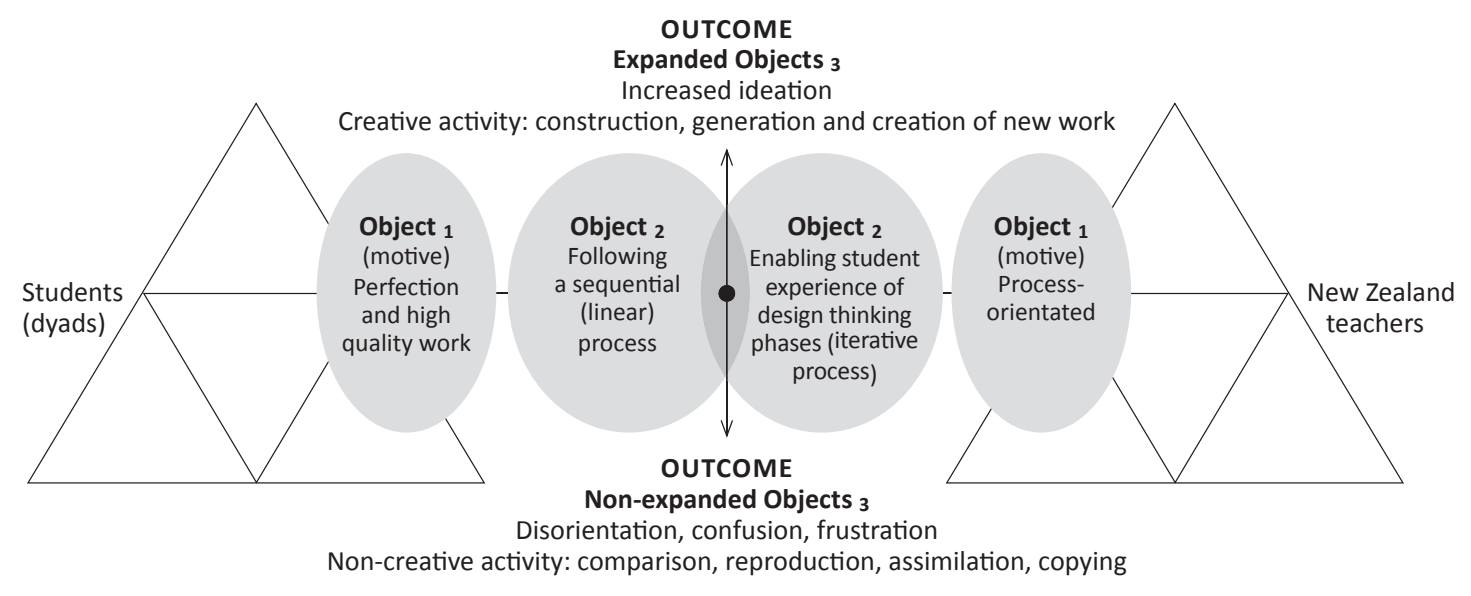

Figure 9.1. Two interacting systems as a minimal model in third generation activity theory (Engeström 2001; Tikhomirov, 1999).

Teasing out the students' motives helped in addressing the sub-questions discussed in the earlier part of this chapter. For most, the collision of previous and current Chinese teaching and learning practices, with imported and unfamiliar educational practices that the New Zealand teachers introduced, led to disorientation, confusion and frustration for the students. Collectively, the dyads were most comfortable using their familiar learning and design work practices which included asking for learning steps and using imitation and repetition. The next figure (Figure 9.2) depicts an inclusion of 
the Chinese teachers' voices. The important thing about this model is that diversity between those perspectives of the students, the Chinese teachers at HNCU and mine as a New Zealand teacher, are represented. Thus, in following Li and Wegerif (2014), an examination of forces related to prior learning and Chinese pedagogical practices has enabled a comprehensive view to be presented.

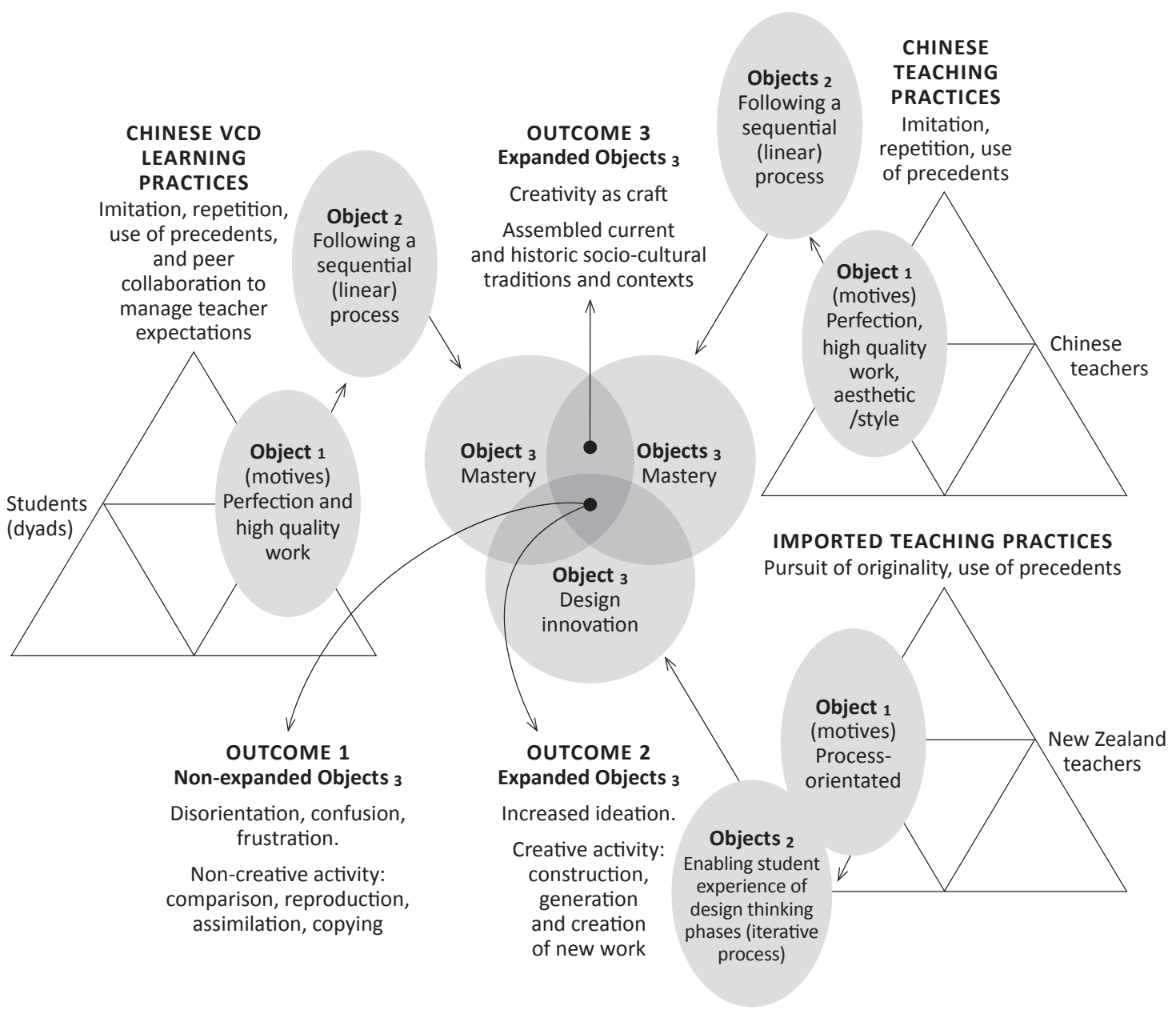

Figure 9.2. A third generation activity theory model to account for multiple voices (Engeström 2001; Tikhomirov, 1999).

In this model, a focus on perfection and high quality design work (product over process) was found to be the most important motive (object 1) for both students and Chinese teachers. Following a sequential (linear) step-by-step process underpinned motives of perfection and high quality design work, and so there are further motives (objects 2) at play. Taking this a step further, there was a divergence in motives between the Chinese and New Zealand teachers. Current instruction of creative design process learning within Chinese classrooms is more likely directed by Chinese teacher 
motives of mastery (represented by object 3 ). This was contradicted by the motives of New Zealand teachers whereby process-oriented and iterative processes constructed through design thinking phases for innovation were in action (object 1, 2 and 3). At this point it is important to note that there are three outcomes from this model. Outcomes one and two evidence the contradictory outcomes that were found through the research and that have been discussed in relation to the two sub-questions and to a certain extent the main research question. However, outcome three presents a different perspective and an opportunity for a new practice and a fresh outlook to materialise from the main research question. Outcome three questions non-creative activity by asking, how can imitiation and repetition be creative? How can something new be discovered from this? And, most importantly, what is the HNCU narrative around these questions?

\section{Creativity as craft}

The way in which pedagogical and student learning practices at HNCU is constructed assembles current and historic sociocultural traditions and contexts. These wider contexts (including prior learning at intensive high school art and design training schools) are central to the creative process, and the relevance of imitation, repetition, use of precedents and peer collaboration are critical for the enablement of learning. I argue that this type of craft creativity draws from and/or assembles such attributes in a way which is unique to this particular social and cultural context (Glăveanu, 2018; Rogoff, 1995). However, rather than perceiving this as corroborating stereotypes of copying and imitation in the literature, it can be viewed quite differently. The practice uses craft in creative ways which enables insight into a local cultural practice that has validity in its own right as opposed to being consigned to comparative judgements which privilege western points of view or ways of doing (Dineen \& Niu, 2008; Glăveanu, 2018).

I argue that a creative craft practice situated within a historic and current socio cultural context is used at HNCU. The practice contains deeply intertwined student objectoriented motives of perfection which follow a sequential (linear) process for the production of high-quality work. Such cultural motives, familiar to the students and 
used by the Chinese teachers to address perceived creativity problems, collided with New Zealand teacher motives which privilege an iterative process in the pursuit of originality and innovation. Recognising this has resulted in the emergence of a new contextual narrative (Glăveanu, 2018). Within this transnational context, a hybrid practice which takes into account the efficacy of this craft practice, alongside New Zealand imported teaching practices which also draws from precedents (artist and designer models) for conceptual development, results in a form of student engagement that holds powerful potential for VCD education in China. Furthermore, enriched intercultural learning environments in which teachers and students are exposed to other ways of doing is crucial for global creative design process learning (Ryan, 2016).

Key to the idea of creativity as craft is that historic and current socio-cultural contexts participate in the creative process and contextual elements such as materiality, and teaching practices which use imitation, repetition and precedents, are assembled. Vygotsky (2004) talked of an organic process which traces and reproduces childhood experiences and impressions in an adaptation of creative or "combinatorial behaviour" in which elements are reworked through the use of imagination or fantasy (p.9). Wartofsky (1979) described a distinctive way of working which captures the essence of craft practice:

The crucial character of the human artefact is that its production, its use, and the attainment of skill in these, can be transmitted, and thus preserved within a social group, and through time, from one generation to the next. The symbolic communication of such skills in the production, reproduction and use of artefacts-i.e. the teaching or transmission of such skills is the context in which mimicry or the imitation of an action becomes a characteristic human mode of activity. (p. 201)

This description of imitation or mimicry echoes Vygotsky's (2004) suggestions that this creative practice may "generate new propositions and new behaviour" (p. 9) which results in humans becoming oriented to the future rather than being limited by a continuous reproduction of old practices. Chuan (2011), a professor at the Soochow University, asserted a specific Chinese cultural perspective: 
Chinese design culture is pluralistic yet holistic; we would not have a healthy Chinese design culture if we ever neglect either ... In its interaction with the outside world, Chinese design culture has been, since ancient times, absorbing and assimilating foreign cultures through various channels while developing its own traditional design culture that is highly eclectic. (p. 129)

These ideas were reflected in the research findings. Amy's experimentation with the circle, a social element (typical seating arrangement when eating) and yin yang cultural elements referenced her intrinsic material-semiotic knowledge (see page 132). Using this internal knowledge as a mediator in her learning, pointed to object-oriented motives which expanded her practice. In a similar way, Michael's dialogue about 'holy high' (see page 150) evidenced an interpretation of object-oriented motive which privileged social, cultural and community elements that were known to him (Engeström \& Miettinen, 1999).

In the process of applying these ideas, a broader literature was engaged which echoes where these ideas were found. Gabora and Tseng (2017) argued that cultural evolution increases when the novelty-generating effects of creativity and imitation are balanced: "imitators ... serve as a "cultural memory" [to] ensure that valuable ideas are preserved" (p. 414). Specifically, from within undergraduate art education, a Japanese study examined the effect of imitation on inspiration and creativity (Okada \& Ishibashi, 2017). Deep encounters with unfamiliar works through processes of copying and imitation were found to enable development and construction of new goals and perspectives in the production of novel work. Okada and Ishibashi likened their findings to the way in which historical artists such as Picasso changed the angles and perspective in his work after being influenced by other artists at that time, thus suggesting that the Cubist movement arose as a form of co-creation. Similarly, Hobbs and Friesem (2019) argue that it was only during the western cultural upheavals of the 1960s that the concept of imitation was met with deep skeptisicm, owing to a belief that creativity and self-expression were denied. Their study found that the popularity of YouTube has resulted in a video remake culture that explicitly imitates or copies original work. Young media makers "develop creative skills through strategic imitation" (Hobbs \& Friesem, 2019, p. 328). These studies challenge the originality 
syndrome, described as an imposed ambition for western design students, as they acknowledge the use of precedents and imitation for learning success (Barr, 2018, p. 1). This in turn, also reflects criticisms that challenge the unconscious illumination phase in creative design process (Amabile, 1996; Howard et al., 2008; Lubart, 1999).

The Chinese teachers at HNCU appeared to be genuinely challenged by creative design process learning, but rather than ignoring the problem they were actively confronting it, and it can be argued the way in which they are succeeding is through promotion of creativity as craft practice. However, overshadowing their efforts are current perspectives from design educators in China that assert both students and teachers alike are highly resistant to criticism that issues in design education in China remain unaddressed, things are at crisis point and student reticence and a refusal to enact change continue to be a problem even though students are aware of the limits of the old teaching methods (Ho et al., 2018). It is concerning that problematic stereotypes may emerge from such statements; to a certain extent, this research has been successful in providing a re-conceptualisation of views about Chinese students and their creativity (Li \& Wegerif, 2014; Mullen, 2017). Although creative design process activities at HNCU may in part be explained by dominant political and social controls which are argued to be limiting (Lin, 2019; Liu \& de Bont, 2017; Zhang, 2017), the research has proposed a local version of creativity learning which takes into account Confucian beliefs and values. This perspective argues that VCD students at HNCU engage in creative design process learning in a creative way that is embedded in craft practices. Furthermore, the creativity of craft cannot be viewed as being any less or more superior to artistic creativity, or the creativity of invention which dominates western ways of thinking about creativity (Glăveanu, 2018). Assertions about the Chinese creativity deficit must be tempered by western cultural expectations and ideals about originality (Barr, 2018; Heng, 2018). Rather than trying to implement a unified definition for a multi-faceted phenomenon, namely creativity and creative design process, adaptation for the Chinese educational context must first ask what type of creativity is being taught, creativity as art, creativity as invention, or creativity as craft? (Glăveanu, 2018). 


\subsection{Epilogue: Crossing cultural boundaries}

When I first taught at HNCU in November 2015 my observations and interactions with a particular pair of students began. Their progress through a design principles course intrigued me owing to the way they were completely immersed in their work through the long class hours. The next time I encountered the two students was during the 2017 delivery of the Miao cultural project. The curriculum was new, and as the students progressed through the design thinking teaching and learning phases, the work these friends produced was impressive. Often, the first to arrive in class, they would show me their work and ask if they were on the right track. The pair had won the Jack playing card which had been designated the Pangfo story which involved a dog, a baby and an emperor. One particular morning, the students produced what I considered to be a superior range of iterations where duplications of dogs and babies had been cut out and arranged in different ways on black paper. There was a curious cog symbol in the body of the dog, and a plinth in the designs, and despite the interpreter's best efforts to explain I could not fully understand and so assumed there was cultural meaning embedded in these motifs. The pair progressed their concept through the ideation and prototype phases to a final outcome. In May 2018 I returned to HNCU to teach the Miao cultural project for the second time. The next pair of students who were working on the Pangfo story included an image of the cog symbol in the dog as a precedent on their mood-board. This image was identical to the representations the two students had produced the year before (see Figure 9.3). It was the first time I saw that image, and it became obvious that the students from the year prior had directly copied the precedent.
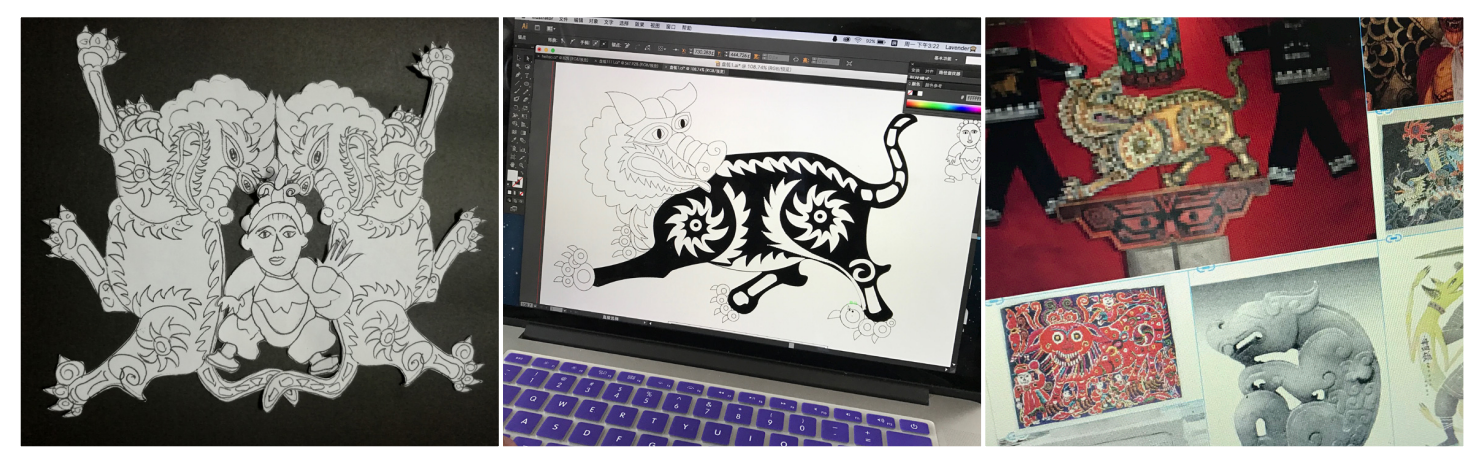

Figure 9.3. Student work in 2017, and the 2018 student mood-board (author images). 
In March 2019, after passing their English IELTS test, the two students were amongst the first cohort to arrive at the higher education institution in New Zealand to complete their degree studies. Their lecturer began their classes by introducing a simple exercise which challenged the students to create a design using only circles and squares in any combination. This exercise was intended to build competency with technical skills and to encourage students to understand how a limited set of components could lead to multiple creative directions. This simple exercise which resulted in highly unique designs by the pair, initiated their year-long major project. The two students continued their collaborative efforts and undertook an extensive idea generation process where they produced a considerable number of sketches to develop a concept (see Figure 9.4).
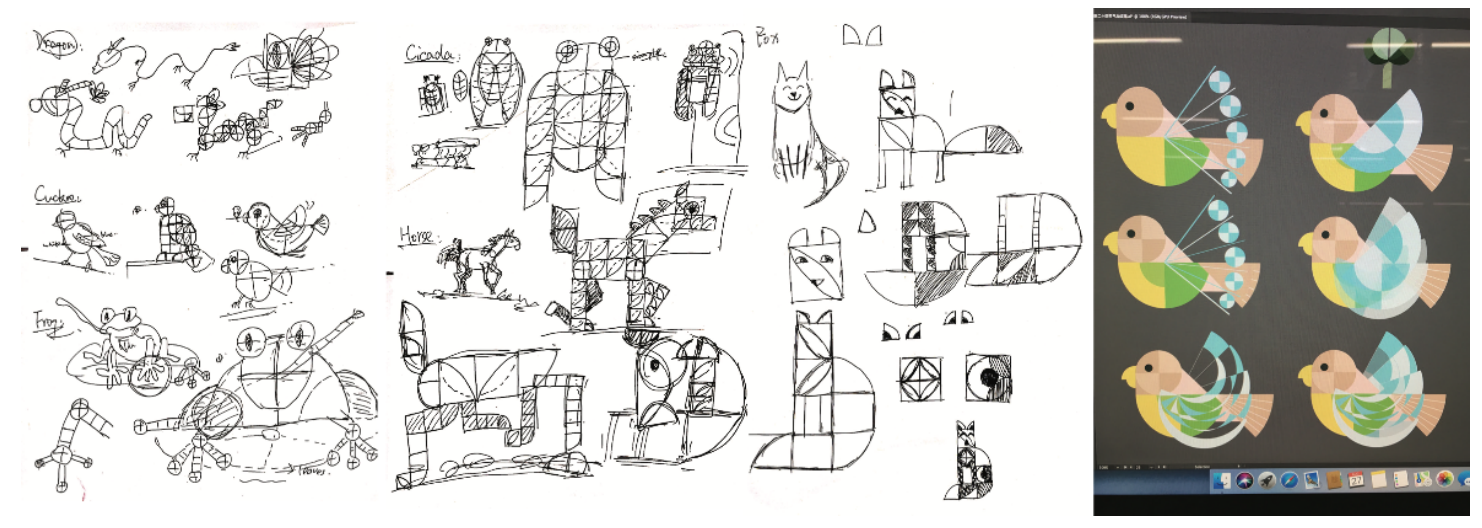

Figure 9.4. Iterative ideation sketches, and computer work using circles to develop the wing of a bird (author images).

Drawing on artist models and the simple shape exercise, the students' process limited the illustration designs to full, half or quarter circles, and they created twenty-four illustrated characters to represent the Chinese solar terms, culminating in a final animation. Whilst idea generation and colour selection were revealed to be a major source of concern for the students in the case studies at HNCU, these two students progressed through the major project by employing self-initiated iterative processes which embedded conceptual meaning for each solar term, as well as confidently employing colour to convey additional meaning (see Figure 9.5). 

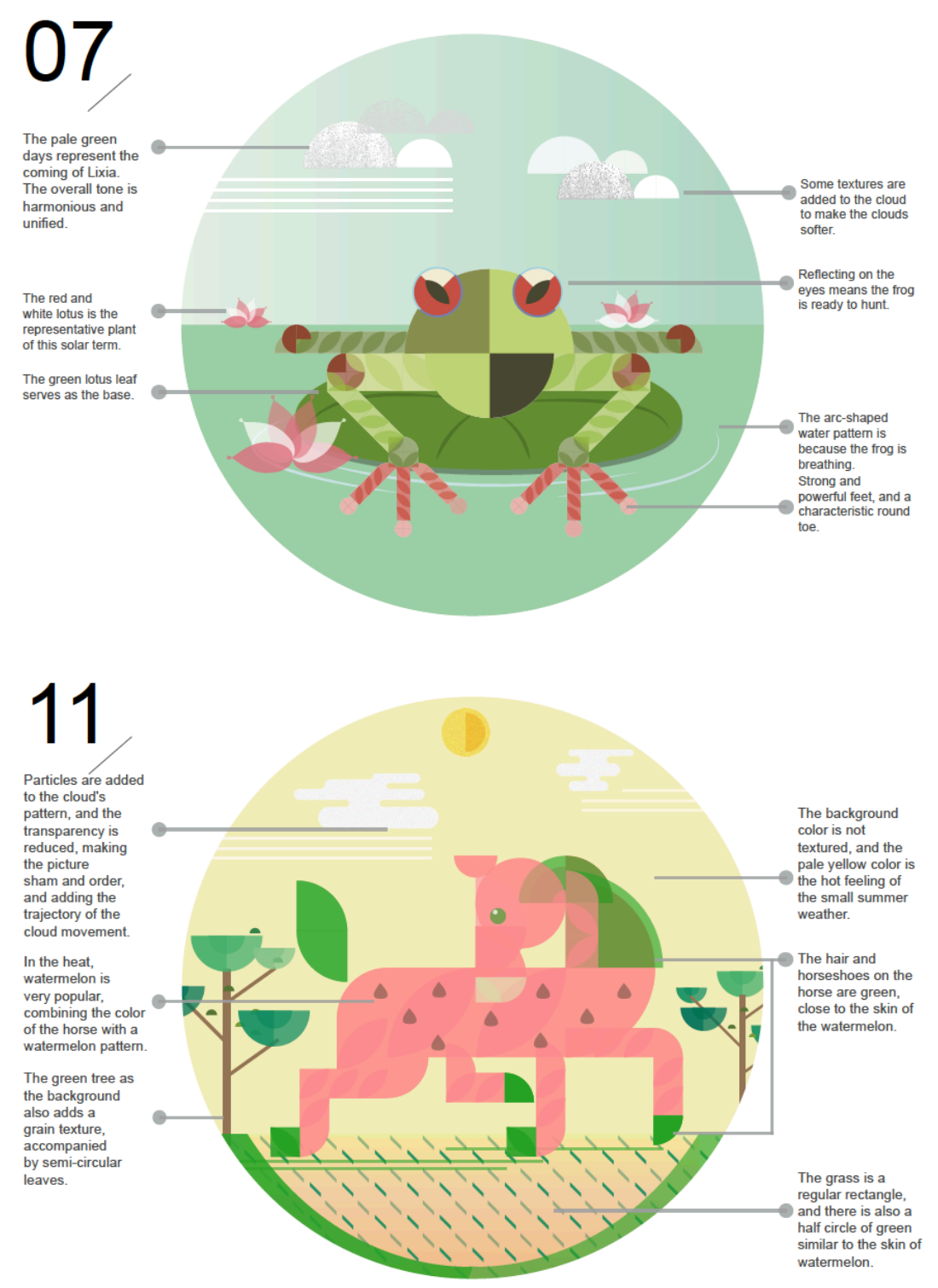

Figure 9.5. Workbook explanations for the way colour and conceptual meanings for the solar terms were embedded into the illustration designs (author images).

This example of two students who crossed cultural boundaries within a particular transnational context highlights the paradox where students from China may become highly conceptual despite an historic way of teaching and learning which is based on transmission approaches of copying and imitation (Li \& Wegerif, 2014). These students countered the research findings in which mediating artefacts such as lack of confidence, frustration, laziness, tiredness, and subjectivity constrained engagement. They came from a Confucian tradition of learning which used craft as creativity in a way that superseded expectations in New Zealand. They began their studies in China 
by using copying and imitation, a practice they were familiar with, and this evolved as they drew on precedents that were visual reproductions of contemporary illustrations. Their shared practice over time echoed transformative and social ways of being, thinking and doing in the production of novel work (Laroche, 2015; Okada \& Ishibashi, 2017; Thulson, 2013). These two students learnt how to generate a significant range of concepts and ideas. After all, communicating an idea in the field of VCD is, "in the end, a more important sign of creativity than the mere ability to represent what already exists" (Buchanan, 2004, p. 36). Their relocation and easy adaptation to the New Zealand educational context reflects Ryan's (2106) assertions that these students were potentially more internationalised than the New Zealand friends that they made. However, the way in which their learning experiences unfold as they transition into professional practice is yet to be seen, which in turn highlights future research directions discussed in the next chapter. 


\section{Chapter 10. Conclusion}

The global reach of transnational education activities, and the way both policy and local preferences have driven change within Sino-foreign co-operations have been studied since 1990 (Qin \& Te, 2016; Yang, 2014a). China is currently positioned within a third wave of a strategy that encourages provision of cooperative TNE programmes with foreign/overseas institutional partners situated within Chinese universities (Yang, 2014a). My research is positioned within this context, specifically, a transnational partnership agreement between Hunan City University (HNCU) in China and a higher education institution in New Zealand.

For design institutions or educators involved in TNE, the significance of the politically motivated 2025 'created in China' strategy, which was introduced in May 2015 by the Central Government in China, should not be underestimated by New Zealand design institutions. China is five years into an ambitious strategic direction, which is to move the country from a manufacturing to an innovation and design-led economy for competitive advantage ( Liu et al., 2018). The global language of VCD is an important component in realising this vision owing to the way VCD by its very nature must communicate concepts and ideas to people within this design-led economy (Buchanan, 2004). Although a student may begin their education specifically in the VCD area, it is common practice for other design fields (for example, architecture or product design) to become an integral part of a design scenario or situation as the novice moves towards expert in professional practice (Buchanan, 1992). To enable understanding about this, design educators, such as myself, use reflective practice within studio-based classrooms (Schön, 1984, 1987) to encourage the shift from analysis to design synthesis (Ledewitz, 1985). Iterative approaches such as design thinking are used in the classroom as the model is promoted as a powerful process which holds potential for design creativity and innovation to emerge (Cross, 2007, 2011; Davis, 2016). Deeply embedded within design activities and design thinking are creative ways of thinking and doing. Creativity is recognised as a critical component in design practice owing to the way it draws on experimentation processes and abilities of fluency and flexibility for problem solving (Orthel, 2015). 
Importing into China these western design and creativity concepts and ways of teaching and learning required affordances of respect towards Yang's (2014a) assertions of "imbalances and asymmetries" (p. 27) within north-south relationships. International education constructed on the basis of western perspectives and historical contexts of colonisation meant problems arising from cultural differences in philosophies and values had to be balanced (Yang, 2014a). This was especially when design educator perspectives from within China asserted that design education is in crisis, owing to a lack of discussion about future directions for the discipline (Ho et al., 2018) and when outside perspectives grapple with the creativity deficit belief and assertions that students from China lack critical thinking skills (Li \& Wegerif, 2014; Mullen, 2017, 2019). Within this context a critical issue relating to philosophical and cultural differences, said to exist between eastern and western creativity, became apparent (Lubart, 1999; Niu, 2012; Niu \& Sternberg, 2006). How do western sociocultural teaching and learning design practices sit alongside Confucian cultural practices? The complex relationship between Confucian values and creative design learning in China emerged as a focus where it has shaped teaching and learning at HNCU.

I turned to cultural historic activity theory (CHAT) and its activity system analytic approach for the theoretical framework, owing to its focus on cultural and historic contexts and the ways in which the complexity of social processes could be captured across different systems (Engeström, 1999, 2001; Leontyev, 2009; Vygotsky, 1978). Theoretically and methodologically, CHAT and its activity system analysis captured the open, fluid, and historically situated nature of VCD students' engagement with creative design process learning at HNCU. AT was paired with case study owing to the compatibilities which are argued to exist (Yamagata-Lynch, 2010). The boundedness of the cultural and historic learning contexts was of interest to the analysis, and case study used to frame the research. The research design comprised two phases. The first phase comprised two projects used as case studies to explore how creative design process learning occurs when the students were exposed to design thinking. Designthinking phases were introduced within the projects, a branding project, and a cultural project involving illustration design. Students were organised into dyads to foster collaborative work. The second phase of the research focused on understanding the 
cultural and historic pedagogical context. Semi-structured interviews were conducted with local teachers at HNCU, and observations were undertaken in Chinese medium classrooms. In total, 24 primary participants were directly involved in both parts of the research and a further 380 secondary participants contributed a range of data for the analysis.

Although the reach of graphic or VCD design is extensive in everyday life, there is virtually no research on VCD creative design process learning from the west, and research conducted in China or the wider Asian region mirrors this limited field of empirical work. As a result, my research questions determined the bodies of literature I have reviewed, including reviews of concepts pertinent to VCD practices. The research questions were focussed on how VCD students engage with creative design process learning at $\mathrm{HNCU}$, and through both the review and the analysis, perspectives from both VCD students and teachers helped to guide my research interpretations.

One of the strengths of this research lies in its temporal nature, which enabled a number of methodological, theoretical and empirical contributions. These contributions are discussed in the next section. Prior to the research being conducted (the nine-week data gathering period spanned one year, from September 2017 to September 2018), I had been embedded in the management, curriculum development and the teaching at HNCU for two years. Since the conclusion of the gathering of research data my involvement with HNCU has continued. Twice a year I have travelled to HNCU to teach new cohorts of VCD students. In combination with this research, my pedagogical practice over this five-year period has been transformed. There were moments of dissonance not only experienced by the students, but also by me; however, my experiences within this transnational context have been profound. By working with wider cohorts of students in both China and New Zealand through this time, I was placed in a unique position to create space for a plurality of perspectives and experiences to be evidenced (Cook-Sather, 2006, 2014). The partnerships between the students and me repositioned my interpretations and observations of creative design process learning at HNCU owing to the way the students became the authors of their practices and processes (Cook-Sather, 2014). Cook-Sather discusses how role transformations "do not entirely dissolve the power relations between older and 
younger researchers" (p. 135); however, to the best of my ability, I believe my research with the VCD students was conceptualised and conducted with an empathetic emphasis which aimed to avoid any hierarchical scenario to emerge. I was sensitive to findings from the literature about morality and hierarchy Confucian code (Chun, 2018; Hung, 2016) in China, and in a multitude of ways this has deeply informed and influenced the way in which the research acknowledges that we were all participants in the research, me, the students, the interpreters, the Chinese teachers and my New Zealand teaching colleagues (Cook-Sather, 2014).

\subsection{Research contributions}

My research contributes sociocultural and historic understandings about VCD students at a particular university in China, situated within the affordances of transnational education. To the best of my knowledge, this research is the first of its kind that uses CHAT and its AS approach to examine how VCD students in an undergraduate university classroom in China engaged with creative design process learning within this transnational context.

For the analysis I drew from Cole (1999), Foot (2014), Wallas (1926), and Wartofsky (1979) to develop a three-level hierarchy of artefacts model (primary, secondary, and tertiary artefacts) to examine the top level of the dyadic activity systems in the branding and cultural projects (see page 64). This methodological contribution came about as I grappled with how a tangible artefact such as a pencil or a computer could be in any way related to intangible feelings or emotion. Many interpretative avenues were opened up as I used the model for the analysis, which in turn highlights the importance of the contribution of my three-level hierarchy of artefacts as it is a taxonomy that extends CHAT for future visual creative scenarios.

The research contributed new insights and original theoretical contributions which have helped re-conceptualise the creativity deficit stereotypes (Li \& Wegerif, 2014). There were three distinct findings that related to my re-conceptualisations. The findings arose from a collision of VCD students', Chinese teachers' and New Zealand 
teachers' objects (motives) which were depicted in a third-generation expression of activity theory (see page 242).

The first finding pointed to similarities in object between both the VCD students and the HNCU teachers. There was a focus on perfection which was interpreted in two ways: the first related to purpose, which in Chinese cultural learning is to attain the highest level of morality within hierarchical structures (Chun, 2018); and the second interpretation of perfection related to something being faultless through actions or the process of improvement.

The first interpretation of perfection was exposed by a complex web of interactions between various stakeholders including parents, and creative pedagogies used in China, such as the high school training programme (see page 116) and use of competitions in the classroom (see page 215). This form of perfection related to Confucian values (Hung, 2016), and intertwined influences of educational reform on design education in China (Barratt-Pugh, et. al., 2018). Both teacher and parental expectations led to significant demands being placed on students, yet the students at HNCU appeared to not question this. They aspired to reach a higher level of morality, good or perfection for the group which when viewed through the lens of activity theory suggests each party played a role in future success when objects were aligned (Leontyev, 1981). The second kind of perfection (high quality work) was evidenced by pedagogies used at HNCU (see chapter 8) in which imitation, repetition, and use of precedents (artist or designer exemplars) which follows a sequential (linear) process pathway were used to attain mastery. Pedagogical practices such as these, in which comparison, reproduction, assimilation and copying are enacted, were attributed to non-creative activity (Engeström, 1999a; Tikhomirov, 1999).

Contradicting this non-creative finding were tensions associated with imported pedagogical practices (see Chapters 6 and 7). The New Zealand teacher objectives were linked to the pursuit of originality. Process-oriented - the design thinking model which relies heavily on iterative practices to achieve design innovation - was used in the classroom. Students who embraced this were attributed as engaging in creative 
activity, owing to the way increased ideation was suggested to result in construction, generation, and creation of new work (Engeström, 1999a; Tikhomirov, 1999).

In following Glăveanu (2018), the dualism of these findings led to a deeper questioning of non-creative activities. How can imitiation and repetition be creative, and what was the HNCU narrative around this? Arising from this examination, I argued that a third type of creativity, which is based on craft, is used at HNCU. This creativity as craft uses guided participation in the development of skill, and practice leads to mastery (for example a mother teaching a child how to sew) (Glăveanu, 2018; Rogoff, 1995). Key to this idea is the way in which sociocultural traditions and the wider context are central to the creative process, and the relevance of imitation, repetition, precedents and mentor support are critical to enable learning. I argue that this type of creativity draws from, and/or assembles such attributes in a way that is unique to a particular social and cultural context. Furthermore, the synergies between creativity as craft and western conceptions, which use "reflection-in-action" (Schön, 1987 , p. 44) in the promotion of studio practices to transition students from novice to expert over time, cannot be denied. The long-held expectation that illumination, believed to appear unexpectedly, and suggestions that serendipity, and combinations of magic and logic are key to originality and new innovation, can be directly challenged owing to the way precedents are both imitated and drawn upon within both New Zealand and Chinese VCD pedagogy (Neumeier, 2005; Wallas 1926). Distinctions became blurred between creative (original) and non-creative (non-original) pedagogical practices, and New Zealand imported and HNCU pedagogies. Essentially, within this particular transnational context, the pedagogical practices of both parties is resulting in an emerging hybrid practice which contains benefits for all (Cook-Sather, 2014; Doherty \& Singh, 2005).

I argue the three findings just discussed contribute a reconceptualisation of creativity deficit stereotypes (Li \& Wegerif, 2014) alongside a deeper understanding about Confucian cultural values in relation to creativity learning within a TNE context (Qin $\&$ Te, 2016). 
My research has some similarities with the work of Dai (2018); and Dai et al. (2020) who suggested cross-system tensions existed for students from China in an Australian TNE digital design course. I have contributed an understanding about a design-based transnational programme that goes beyond the hands-off scenario described by Dai et al. (2020). Occasional visits to partner institutions by guest lecturers, who provided not much more than an introduction to their country of origin, led to "learning shocks" and barriers when students changed educational institutions (Dai, 2018, p. 12). This body of research contains a salient message for institutions and lecturers who engage in TNE arrangements. Relationships, so central to Chinese culture, cannot be underestimated when intentions and promises made at the time of signing a transnational agreement do not flow through to the support of student transfer (WilsonMah \& Thomlinson, 2018).

Finally, my research has contributed to the dearth of research about the enagement of graphic, VCD or communication design education in general (Heller, 2015; Walker, 2017), and the potential influence of this particular design discipline for economic advancement in China. The two case studies, a branding and a cultural illustration project (see Appendices 6.1., page 286 and 7.1., page 296) highlighted a studio-based practice (Schön, 1987). Within the pedagogy, design thinking phases were used, and creativity was recognised as an important component within design practice owing to the way it draws on experimentation processes and abilities of fluency and flexibility for problem solving (Orthel, 2015). This contribution addresses the significant lack of western research which connects design thinking specifically to VCD education, and it has built on the two Korean studies which applied a design thinking process within the curriculum (Kim \& Lee, 2016; Lee, 2019).

\subsection{Limitations and possibilities for further research}

It is suggested a combined role in research may lead to valuable inside views (Creswell, 2013). Being both teacher and observer at the same time in the case studies had its difficulties owing to the inherent teaching distractions involving other students, which in turn led to some difficulties in data collection. However, my exposure to the VCD students at HNCU over a sustained period went beyond the data-gathering 
phases. Critical discussions with colleagues and supervisors took place through this time, along with many personal observations of learning when I was a teacher rather than a researcher. I understood that each participant was unique, and their motives were different, thus limiting any kind of generalised conjecture for future teaching (Disarro 2014). Through my self-reflexive practice, the research aimed to present an account enriched with thick descriptions of complex creative design process relations, interactions and issues within current and historic contexts (Geertz, 1973; Merriam, 1998; Stake, 1995).

As I do not speak Mandarin, the veracity of the translations cannot be guaranteed. This limitation was discussed in Chapter 5 (see page 123). Ethically and methodologically it was my responsibility to manage and reflect upon issues of cross-language nuance, hierarchies of language power, and situated language epistemologies during analysis and interpretation of the transcriptions (Temple, 2008; Temple \& Young, 2004). Future studies may usefully draw on my findings in order to contribute a growing research base to inform future transnational policies and education delivery.

From my particular research participants, future longitudinal research possibilities are evident. In particular, I have been observing the first two cohorts since 2015. Participants for my research were drawn from the second cohort of VCD students who are still enrolled at HNCU. The two students discussed in the epilogue (see page 248) came from the first cohort of students I taught; these students have now completed their undergraduate studies. Future study is required to understand how their transnational learning experiences unfold as they transition to professional practice. In particular, further research is needed to understand how transnational higher education influences: (1) students' readiness for professional practice; (2) where students choose to work, do they stay in their adopted country, or do they return to China? (3) Ryan's (2106) assertions that these students were potentially more internationalised; and (4) transitions into postgraduate pathways.

My findings open up further avenues for study that relate to the notion of developing TNHE hybrid practices and/or balancing the novelty-generating effects of creativity with practices of copying and imitation in China (Gabora \& Tseng, 2017). A wider 
literature was consulted about this (Barr, 2018; Hobbs \& Friesem, 2019; Okada \& Ishibashi, 2017); however, it is limited to creativity disciplines other than graphic or VCD education. Therefore, future research should include a literature review to understand to what extent current conversations are taking place about this and in which creativity disciplines this is common practice.

Furthermore, it would be beneficial to conduct emperical research at other higher educational sites in China to contrast my findings, in particular with regard to: (1) student accessability to TNHE design education opportunities which were raised as problematic for students from poorer regions in China (Marginson, 2018; Montgomery, 2016); (2) Chinese teacher accessability to professional development opportunities within TNHE design education initiatives in order to foster indigenous design thinking approaches tailored to the China context (Buchanan, 2004), and to address concerns about design education in China (Ho, Li, Liu \& Wang, 2018); and (3) future collaborations between New Zealand and/or other teacher nationalities and Chinese teachers in joint TNHE design education classrooms to encourage rich intercultural exchanges (Caruana \& Montgomery, 2015). The last point was a personal highlight of my research. Confucian values and characteristics shed light on practices and student behaviour that were foreign to me whilst undertaking this research. There was a point when I discovered that within Confucianism a certain type of creativity exists; it draws on social interaction and codes of hierarchy and morality (Chun, 2018). Understanding the importance of this highlights the epistemological significance of my research. This insight also offers an alternate perspective on ways of thinking and doing that holds validity and the potential for different forms of student innovation to emerge (Buchanan, 2004).

Finally, I return to the New Zealand government international education strategy discussed at the beginning of this thesis (NZ Government, 2018). My research evidences a practical studio-based pedagogy delivered in person in China (Schön, 1987). The New Zealand government strategy also calls for explorations and opportunities to deliver online educational options. The limitations associated with delivering practical course material in an online environment, relates to a studio-based practice that relies on forged relationships between teachers and students in which 
demonstrations and language help build understanding (Schön, 1987). However, at the time of writing, owing to the Covid-19 virus, travel to China is suspended and opportunities for online delivery within the TNHE context are being explored by myself and my colleagues. Future research is required to ascertain how online creativity learning delivery potentially influences: (1) student engagement; (2) creative design process teaching and learning; (3) student enactment within the three-level hierarchy of artefacts; and (4) the tensions and contradictions that exist in activity system models within a TNHE-technological context. 


\section{References}

Aaker, D. A., \& Joachimsthaler, E. (2000). Brand leadership. New York, NY: Free Press, Simon \& Schuster.

Amabile, T. M. (1983). The social psychology of creativity: A componential conceptualization. Journal of Personality and Social Psychology, 45(2), 357-376.

Amabile, T. M. (1996). Creativity in context. Boulder, Colorado: Waterview Press.

Appiah, E., \& Cronjé, J. (2013). Information communication and technology (ICT) and the challenges of ideation in graphic design: An activity theory focus. International Journal of Computer Applications, 63(6), 13-23. https://doi.org/10.5120/104695192

Archer, L. B. (1979). Design as a discipline: Whatever became of design methodology? The three Rs. Design Studies, 1(1), 17-20. http://dx.doi.org/10.1016/0142694X(79)90023-1

Arnold, J. E. (2016). Creative engineering. In W. J. Clancey (Ed.), Creative engineering: Promoting innovation by thinking differently (pp. 50-150). Retrieved from Stanford Digital Repository. http:// purl.stanford.edu/jb100vs5745.

Barnbrook, J., Kalman, T., Lupton, E., McCoy, K., Poynor, R., Miller, A., ... Spiekermann, E. (1999). First things first manifesto 2000. Retrieved from http://www.eyemagazine.com/feature/article/first-things-first-manifesto-2000

Barr, A. (2018). Alistair Barr: Originality has become the imposed ambition for architecture students, but we should all embrace the exhilarating possibilities of creative copying. Blueprint (London, England), (360), 21. Retrieved from https://search-proquestcom.helicon.vuw.ac.nz/docview/2124123544?accountid=14782

Barratt-Pugh, L., Zhao, F., Zhang, Z., \& Wang, S. (2018). Exploring current Chinese higher education pedagogic tensions through an activity theory lens. Higher Education, 77(5), 831-852. http://dx.doi.org/10.1007/s10734-018-0304-8

Bierut, M. I. (2000). A manifesto. The International Design Magazine; New York, 47(2), 76-79. Retrieved from https://search.proquest.com/docview/220297257?accountid=12834

Biesta, G. (2016). Beautiful risk of education. London: Routledge.

Biesta, G. J. (2012). Giving teaching back to education: Responding to the disappearance of the teacher. Phenomenology \& Practice, 6(2), 35-49. http://dx.doi.org/10.29173/pandpr19860

Bloom, B. S. (1953). Thought processes in lectures and discussions. The Journal of General Education, 7(3), 160-169. Retrieved from http://www.jstor.org/stable/27795429

Bourke, R., Mentis, M., \& O’Neill, J. (2013). Using activity theory to evaluate a professional learning and development initiative in the use of narrative assessment. Cambridge Journal of Education, 43(1), 35-50. https://doi.org/10.1080/0305764X.2012.749214 
Brenner, W., \& Uebernickel, F. (Eds.). (2016). Design thinking for innovation: Research and practice. Cham: Springer. https://doi.org/10.1007/978-3-319-26100-3

Brown, T. (2008, June). Design thinking. Harvard Business Review, 85-92.

Brown, T., \& Rowe, P. G. (2008). Design thinking. Harvard Business Review, 86(6), 252. https://doi.org/10.5437/08956308X5503003

Buchanan, R. (1992). Wicked problems in design thinking. Design Issues, 8(2), 5-21. Retrieved from http://www.jstor.org/stable/1511637

Buchanan, R. (2004). Human-centered design: Changing perspectives on design education in the east and west. Design Issues, 20(1), 30-39. http://dx.doi.org/10.1162/074793604772933748

Caretta, M. A. (2015). Situated knowledge in cross-cultural, cross-language research: A collaborative reflexive analysis of researcher, assistant and participant subjectivities. Qualitative Research, 15(4), 489-505. https://doi.org/10.1177/1468794114543404

Caruana, V., \& Montgomery, C. (2015). Undertstanding the transnational higher education landscape: Shifting positionality and the complexities of partnership. Learning and Teaching: The International Journal of Higher Education in the Social Sciences, 8(1), 5-29. http://dx.doi.org/10.3167/latiss.2015.080102

Ceppi, S. (2017). Critical thinking and design education in China: Considerations from a western perspective. In K. W. M. Siu \& J. Contreras, G (Eds.), Design education for fostering creativity and innovation in China. Hershey, PA: IGI Global.

Chuan, Z. P. (2011). Regional traits and mobility of the design culture in ancient China and the starting point for design education in contemporary China. Iridescent, 1(1), 118-129. https://doi.org/10.1080/19235003.2011.11782250

Chun, W. (2018). Chinese philosophy. Shanghai: China Intercontinental Press.

Cole, M. (1999). Cultural psychology: Some general principles and a concrete example. In Y. Engeström, R. Miettinen, \& R.-L. Punamaki (Eds.), Perspectives on activity theory (pp. 87-106). Cambridge, MA: Cambridge University Press.

Confucius. (1954). The wisdom of Confucius. In S. Commins \& R. N. Linscott (Eds.), Man and man: The social philosophers (pp. 329-364). New York: Modern Pocket Library.

Cook-Sather, A. (2006). Education is translation: A metaphor for change and teaching. Philadelphia: University of Pennsylvania Press.

Cook-Sather, A. (2014). The trajectory of student voice in educational research. New Zealand Journal of Educational Studies, 49(2), 131-148.

Creswell, J. W. (2013). Qualitative inquiry \& research design: Choosing among five approaches $\left(3^{\text {rd }}\right.$ ed.). Thousand Oaks, CA: Sage

Cross, N. (2007). Designerly ways of knowing. Basel, Switzerland: Burkhauser.

Cross, N., Naughton, J., \& Walker, D. (1981). Design method and scientific method. Design Studies, 2(4), 195-201. http://dx.doi.org/10.1016/0142-694X(81)90050-8

Crow, D. (2003). Visible signs. Crans-près-Céligny, Switzerland: AVA Academia.

Csikszentmihalyi, M. (1990). Flow: The psychology of optimal experience. New York, NY: Harper \& Row. 
Csikszentmihalyi, M. (2014). The systems model of creativity. The collected works of Mihaly Csikszentmihalyi. Dordrecht: Springer. https://doi.org/10.1007/978-94-0179085-7

Dai, K. (2018). Learning between two systems: A Chinese student's reflexive narrative in a China-Australia articulation programme. Compare, online, 1-20. https://doi.org/10.1080/03057925.2018.1515008

Dai, K., Matthews, K. E., \& Renshaw, P. (2020). Crossing the 'bridges' and navigating the 'learning gaps': Chinese students learning across two systems in a transnational higher education programme. Higher Education Research and Development, online, 1-15. https://doi.org/10.1080/07294360.2020.1713731

Daniels, H., Cole, M., \& Wertsch, J. V. (Eds.). (2007). The Cambridge companion to Vygotsky ( $2^{\text {nd }}$ ed.). New York: Cambridge University Press.

Davis, M. (2016). "Normal science" and the changing practices of design and design education. Visible Language, 50(1), 1-7.

Dewey, J. (1934). Art as experience. New York: Penguin Group.

Dewey, J. (1938). Experience and education. New York, NY: Free Press, Simon \& Schuster.

Dineen, R., \& Niu, W. (2008). The effectiveness of western creative teaching methods in China: An action research project. Psychology of Aesthetics, Creativity, and the Arts, 2(1), 42-52. https://doi.org/10.1037/1931-3896.2.1.42

Disarro, D. R. (2014). Let's CHAT: Cultural historical activity theory in the creative writing classroom. New Writing, 11(3), 438-451. https://doi.org/10.1080/14790726.2014.956123

Doherty, C., \& Singh, P. (2005). How the West is Done: Simulating Western Pedagogy in a Curriculum for Asian International Students. In P. Ninnes, \& M. Hellstén (Eds.), Internationalizing Higher Education: Critical Explorations of Pedagogy and Policy (pp. 53-73). Hong Kong: Comparative Education Research Centre of The University of Hong Kong and Kluwer Academic Publishers.

Don, C. (2014). From "Made in China" to "Designed in China." Paper presented at the M+ Matters, Hong Kong. Retrieved from www.mplusmatters.hk/importexport/pdf/herenke.pdf.

Dorst, K. (2011). The core of "design thinking” and its application. Design Studies, 32(6), 521-532. https://doi.org/10.1016/j.destud.2011.07.006

Dorst, K., \& Cross, N. (2001). Creativity in the design process: Co-evolution of problemsolution. Design Studies, 22(5), 425-437. https://doi.org/10.1016/S0142694X(01)00009-6

Doyle, S. (2016). Changing flows and directions for international education and mobilities in selected Asian countries: Turning to look at Asia from a New Zealand perspective. New Zealand Journal of Asian Studies, 18(2), 13-28.

Edwards, R. (1998). A critical examination of the use of interpreters in the qualitative research process. Journal of Ethnic and Migration Studies, 24(1), 197-208. https://doi.org/10.1080/1369183X.1998.9976626

Engeström, Y. (1987). Learning by expanding: An activity-theoretical approach to developmental research. Helsinki: Orienta-Konsultit. 
Engeström, Y. (1993). Development studies of work as a testbench of activity theory: The case of primary care medical practice. In S. Chaiklin \& J. Lave (Eds.), Understanding practice: Perspectives on activity and context (pp. 64-103). New York: Cambridge University Press.

Engeström, Y. (1999). Activity theory and individual and social transformation. In Y. Engeström, R. Miettinen, \& R. L. Punamaki (Eds.), Perspectives on activity theory (pp. 19-38). Cambridge, UK: Cambridge University Press.

Engeström, Y. (1999a). Innovative learning in work teams: Analyzing cycles of knowledge creation in practice. In Y. Engeström, R. Miettinen, \& R. L. Punamaki (Eds.), Perspectives on activity theory (pp. 377-404). Cambridge: Cambridge University Press.

Engeström, Y. (2001). Expansive learning at work: Toward an activity theoretical reconceptualization. Journal of Education and Work, 14(1), 133-156. https://doi.org/10.1080/13639080020028747

Engeström, Y. (2005). Developmental work research: Expanding activity theory in practice. Berlin: Lehmanns Media.

Engeström, Y., \& Glăveanu, V. (2012). On third generation activity theory: Interview with Yrjö Engeström. Europe's Journal of Psychology, 8(4), 515-518. https://doi.org/10.5964/ejop.v8i4.555

Engeström, Y., \& Miettinen, R. (1999). Introduction. In Y. Engeström, R. Miettinen, \& R.-L. Punamaki (Eds.), Perspectives on activity theory (pp. 1-18). Cambridge, UK: Cambridge University Press.

Fontaine, L. (2014). Learning design thinking by designing learning experiences: A case study in the development of strategic thinking skills through the design of interactive museum exhibitions. Visible Language, 48(2), 49-69.

Foot, K. A. (2014). Cultural-Historical Activity Theory: Exploring a theory to inform practice and research. Journal of Human Behavior in the Social Environment, 24(3), 329-347. https://doi.org/10.1080/10911359.2013.831011

Gabora, L., \& Tseng, S. (2017). The social benefits of balancing creativity and imitation: Evidence from an agent-based model. Psychology of Aesthetics, Creativity, and the Arts, 11(4), 403-419. https://doi.org/10.1037/aca0000132

Garner, S., \& McDonagh-Philp, D. (2001). Problem interpretation and resolution via visual stimuli: The use of "mood boards" in design education. Journal of Art and Design Education, 20(1), 57-64. https://doi.org/10.1111/1468-5949.00250

Geertz, C. (1973). Thick description: Toward an interpretive theory of culture. In C. Geertz (Ed.), The interpretation of cultures; selected essays (pp. 3-30). New York: Basic Books.

Glăveanu, V. P. (2018). Educating which creativity? Thinking Skills and Creativity, 27(October 2017), 25-32. https://doi.org/10.1016/j.tsc.2017.11.006

Gobé, M. (2009). Emotional branding. New York, NY: Allworth Press.

Goffman, E. (1955). On face-work: An analysis of ritual elements in social interaction. Psychiatry, 18(3), 213-231. 
Gourlay, L. (2017). Student engagement, "Learnification" and the sociomaterial: Critical perspectives on higher education policy. Higher Education Policy, 30(1), 23-34. https://doi.org/10.1057/s41307-016-0037-1

Gropius, W. (1956). Scope of total architecture. London: George Allen \& Unwin.

Guba, E. G. (1987). What have we learned about naturalistic evaluation? American Journal of Evaluation, 8(1), 23-43. https://doi.org/10.1177/109821408700800102

Guilford, J. P. (1956). The structure of intellect. Psychological Bulletin, 53(4), 267-293. https://doi.org/10.1037/h0040755

Hardman, J., \& Amory, A. (2015). Introduction to cultural-historical activity theory and tool mediation. In V. Bozalek, D. Ng'ambi, D. Wood, J. Herrington, J. Hardman, \& A. Amory (Eds.), Activity theory, authentic learning and emerging technologies: Towards a transformative higher education pedagogy (pp. 9-21). London: Routledge. https://doi.org/10.4324/9781315771823

He, L., \& Liu, E. (2018). Cultural influences on the design and management of transnational higher education programs in China: A case study of three programs. International Journal of Educational Management, 32(2), 269-283. https://doi.org/10.1108/IJEM-02-2017-0044

He, M., \& Zhang, S. (2011). Re-conceptualizing the Chinese concept of face from a facesensitive perspective: A case study of a modern Chinese TV drama. Journal of Pragmatics, 43(9), 2360-2372. https://doi.org/10.1016/j.pragma.2011.03.004

Healey, N. M. (2015). Towards a risk-based typology for transnational education. Higher Education, 69(1), 1-18. https://doi.org/10.1007/s10734-014-9757-6

Heller, S. (Ed.). (2015). The education of a graphic designer ( $3^{\text {rd }}$ ed.). New York: Allworth Press.

Heng, T. T. (2018). Different is not deficient: Contradicting stereotypes of Chinese international students in US higher education. Studies in Higher Education, 43(1), 22-36. https://doi.org/10.1080/03075079.2016.1152466

Hilppö, J., Lipponen, L., Kumpulainen, K., \& Rajala, A. (2017). Visual tools as mediational means: A methodological investigation. Journal of Early Childhood Research, 15(4), 359-373. https://doi.org/10.1177/1476718X15617795

Hilton, C. (2016). How language limitations affect conceptual thresholds among Chinese design students in the United Kingdom. Art, Design and Communication in Higher Education, 15(2), 135-143. https://doi.org/10.1386/adch.15.2.135_1

Ho, J., Li, D., Liu, Y., \& Wang, Y. (2018). Thoughts on design education in China: From classroom to workshop. Landscape Architecture Frontiers, 6(5), 80-85. https://doi.org/10.15302/j-laf-20180508

Hobbs, R., \& Friesem, Y. (2019). The creativity of imitation in remake videos. E-Learning and Digital Media, 16(4), 328-347. https://doi.org/10.1177/2042753019835556

Hong, T. P. T. (2011). Issues to consider when implementing student-centred learning practices at Asian higher education institutions. Journal of Higher Education Policy and Management, 33(5), 519-528. https://doi.org/10.1080/1360080X.2011.605226

Howard, A. (1994). There is no such thing as society. Retrieved from http://www.eyemagazine.com/feature/article/there-is-such-a-thing-as-society 
Howard, T. J., Culley, S. J., \& Dekoninck, E. (2008). Describing the creative design process by the integration of engineering design and cognitive psychology literature. Design Studies, 29(2), 160-180. https://doi.org/10.1016/j.destud.2008.01.001

Hu, M., \& Willis, L. D. (2017). Towards a common transnational education framework: Peculiarities in China matter. Higher Education Policy, 30(2), 245-261. https://doi.org/10.1057/s41307-016-0021-9

Hung, R. (2016). A critique of Confucian learning: On learners and knowledge. Educational Philosophy and Theory, 48(1), 85-96. https://doi.org/10.1080/00131857.2015.1084220

Illiprandi, G. (1991). Charter of graphic design proposal for a debate on visual communication design. Design Issues, 8(1), 67-73. https://doi.org/10.2307/1511455

Ings, W. (2015). Malleable thought: The role of craft thinking in practice-led graphic design. International Journal of Art \& Design Education, 34(2), 180-191. https://doi.org/10.1111/jade.12013

Jain, N. K., Celo, S., \& Kumar, V. (2019). Internationalization speed, resources and performance: Evidence from Indian software industry. Journal of Business Research, 95(February 2018), 26-37. https://doi.org/10.1016/j.jbusres.2018.09.019

Jensen, R. (1999). The dream society: How the coming shift from information to imagination will transform your business. New York, NY: McGraw-Hill.

Jiang, H., Tang, M. X., Peng, X., \& Liu, X. (2018). Learning design and technology through social networks for high school students in China. International Journal of Technology and Design Education, 28(1), 189-206. https://doi.org/10.1007/s10798016-9386-8

Johnson, W. H. A., \& Weiss, J. W. (2011). Into the heart of the dragon; An introduction and agenda on innovation and education towards innovation in China. Journal of Technology Management in China, 6(3), 196-202. https://doi.org/10.1108/17468771111157418

Johnson, W. H., \& Weiss, J. W. (2008). A stage model of education and innovation type in China: The paradox of the dragon. Journal of Technology Management in China, 3(1), 66-81. https://doi.org/10.1108/17468770810851502

Jones, R. (2010). Fail again: Fail better. In Jacob, M. J., \& Bass, J (Eds.), Learning mind: Experience into art (pp. 150-163). Oakland: University of California Press.

Kaptelinin, V. (2005). The object of activity: Making sense of the sense-maker. Mind, Culture, and Activity, 12(1), 4-18. https://doi.org/10.1207/s15327884mca1201_2

Kaufman, J. C., \& Beghetto, R. A. (2009). Beyond big and little: The four C model of creativity. Review of General Psychology, 13(1), 1-12. https://doi.org/10.1037/a0013688

Keane, M. (2006). From made in China to created in China. International Journal of Cultural Studies, 9(3), 285-296. https://doi.org/10.1177/1367877906066875

Kember, D. (2016). Understanding and teaching the Chinese learner: Resolving the paradox of the Chinese learner. In R. B. King \& A. B. I. Bernardo (Eds.), The psychology of Asian learners: A festschrift in honor of David Watkins (pp. 173187). Singapore: Springer. https://doi.org/10.1007/978-981-287-576-1_11 
Kim, H., \& Lee, H. (2016). Cognitive activity-based design methodology for novice visual communication designers. International Journal of Art \& Design Education, 35(2), 196-212. https://doi.org/10.1111/jade.12054

Kimbell, L. (2011). Rethinking design thinking: Part I. Design and Culture. The Journal of the Design Studies Forum, 3(3), 285-306. https://doi.org/10.2752/175470812X13281948975413

Knight, J. (2003). Updating the definition of internationalization. International Higher Education, 33(Fall), 2-3.

Knight, J. (2016). Transnational education remodeled: Toward a common TNE framework and definitions. Journal of Studies in International Education, 20(1), 34-47. https://doi.org/10.1177/1028315315602927

Kolb, D. (1984). Experiential learning: Experience as the source of learning and development. Englewood Cliffs, New Jersey: Prentice-Hall.

Kosny, A., MacEachen, E., Lifshen, M., \& Smith, P. (2014). Another person in the room: Using interpreters during interviews with immigrant workers. Qualitative Health Research, 24(6), 837-845. https://doi.org/10.1177/1049732314535666

Krippendorff, K. (2004). Intrinsic motivation and human-centred design. Theoretical Issues in Ergonomics Science, 5(1), 43-72. https://doi.org/10.1080/1463922031000086717

Kvale, S. (2006). Dominance through interviews and dialogues. Qualitative Inquiry, 12(3), 480-500. https://doi.org/10.1177/1077800406286235

Laing, S., \& Masoodian, M. (2016). A study of the influence of visual imagery on graphic design ideation. Design Studies, 45, 187-209. https://doi.org/10.1016/j.destud.2016.04.002

Laroche, G. A. (2015). Social learning and drawing: What children learn by copying the images of their peers. Art Education, 68(3), 19-25. https://doi.org/10.1080/00043125.2015.11519318

Lave, J. (1993). The practice of learning. In S. Chaiklin \& J. Lave (Eds.), Understanding practice: Perspectives on activity and context (pp. 3-34). New York: University of Cambridge.

Ledewitz, S. (1985). Models of design in studio teaching. Journal of Architectural Education, 38(2), 2-8.

Lee, H. (2019). Revitalising traditional street markets in rural Korea: Design thinking and sense-making methodology. International Journal of Art \& Design Education, 38(1), 256-269. https://doi.org/10.1111/jade.12183

Lee, J. H., Portillo, M., \& Meneely, J. (2019). Insights into three frames of creative minds: Igniting perspective transformation among first-year university students. Journal of Transformative Education, online, 1-25. https://doi.org/10.1177/1541344619893314

Leontiev, A. N. (2005). The genesis of activity. Journal of Russian and East European Psychology, 43(4), 58-71.

https://doi.org/http://dx.doi.org/10.1080/10610405.2005.11059253

Leontyev, A. N. (1981). Problems of the development of the mind. Moscow: Progress Publishers.

Leontyev, A. N. (2009). Activity and consciousness. In Mike Cole (Ed.), The development 
of mind: Selected works of Aleksei Nikolaevich Leontyev (pp. 395-419). CA:

Marxists Internet Archive. Retrieved from

https://www.marxists.org/admin/books/activity-theory/leontyev/developmentmind.pdf

Li, L., \& Wegerif, R. (2014). What does it mean to teach thinking in China? Challenging and developing notions of "confucian education." Thinking Skills and Creativity, 11, 22-32. https://doi.org/10.1016/j.tsc.2013.09.003

Lin, J. (2019). ( Un-) becoming Chinese creatives: transnational mobility of creative labour in a 'global' Beijing. Mobilities, 14(4), 452-468. https://doi.org/10.1080/17450101.2019.1571724

Lincoln, Y. S., \& Guba, E. G. (2011). Paradigmatic controversies, contradictions, and emerging confluences. In N. K. Denzin \& Y. S. Lincoln (Eds.), The Sage handbook of qualitative research ( $4^{\text {th }}$ ed., pp. 163-188). Thousand Oaks, CA: Sage.

Liu, S. X. (2016). Innovation design: Made in China 2025. Design Management Review, 27(1), 52-58. https://doi.org/10.1007/978-1-4471-2268-5

Liu, S. X., \& de Bont, C. (2017). Barriers to strategic design: A perspective from China. The Journal of Design, Economics, and Innovation, 3(2), 133-145. https://doi.org/10.1016/j.sheji.2017.09.003

Liu, S. X., Liu, H., \& Zhang, Y. (2018). The new role of design in innovation: A policy perspective from China. The Design Journal, 21(1), 37-58. https://doi.org/10.1080/14606925.2017.1395167

Lo, A. (2000). The game of leaves: An inquiry into the origin of Chinese playing cards. Bulletin of the School of Oriental and African Studies, University of London, 63(3), 389-406.

Logan, C. (2007). Metaphor and pedagogy in the design practicum. International Journal of Technology and Design Education, 18(1), 1-17. https://doi.org/10.1007/s10798006-9009-x

Lubart, T. I. (1999). Creativity across cultures. In R. J. Sternberg (Ed.), Handbook of creativity (pp. 339-350). Cambridge, UK: Cambridge University Press.

Lyle, J. (2003). Stimulated recall: A report on its use in naturalistic research. British Educational Research Journal, 29(6), 861-878. https://doi.org/10.1080/0141192032000137349

Mackey, A., \& Grass, S. M. (2015). Second language research: Methodology and design (2nd ed.). Florence, US: Routledge. Retrieved from www.ebrary.com.helicon.vuw.ac.nz

Madge, C., \& Noxolo, P. (2015). Conceptualizing international education: From international student to international study. Progress in Human Geography, 39(6), 681-701. https://doi.org/10.1177/0309132514526442

Marginson, S. (2000). Rethinking academic work in the global era. Journal of Higher Education Policy and Management, 22(1), 23-35. https://doi.org/10.1080/713678133

Marginson, S. (2018). Higher education, economic inequality and social mobility: Implications for emerging East Asia. International Journal of Educational Development, 63, 4-11. https://doi.org/10.1016/j.ijedudev.2017.03.002 
Merriam, S. B. (1998). Qualitative research and case study applications in education. San Francisco, CA: Jossey-Bass.

Merriam, S. B. (2009). Qualitative research: A guide to design and implementation: Revised and expanded from qualitative research and case study applications in education ( $3^{\text {rd }}$ ed.). San Francisco, CA: Jossey-Bass.

Mok, K. H., \& Han, X. (2016). The rise of transnational higher education and changing educational governance in China. International Journal of Comparative Education and Development, 18(1), 19-39. https://doi.org/10.1108/ijced-10-2015-0007

Montgomery, C. (2016). Transnational partnerships in higher education in China: The diversity and complexity of elite strategic alliances. London Review of Education, 14(1), 70-85. https://doi.org/10.18546/LRE.14.1.08

Moran, S., \& John-Steiner, V. (2003). Creativity in the making: Vygotsky's contemporary contribution to the dialectic of development and creativity. In R. K. Sawyer, V. John-Steiner, S. Moran, R. J. Sternberg, D. H. Feldman, J. Nakamura, \& M. Csikszentmihalyi (Eds.), Creativity and development (pp. 61-90). New York, NY: Oxford University Press. Retrieved from http://site.ebrary.com.helicon.vuw.ac.nz/lib/vuw/reader.action?docID=10085259

Mullen, C. A. (2017). Creativity and education in China: Paradox and possibilities for an era of accountability. New York: Routledge.

Mullen, C. A. (2019). Do Chinese learners have a creativity deficit? Kappa Delta Pi Record, 55(3), 100-105. https://doi.org/10.1080/00228958.2019.1622373

Naidoo, K., \& Kirch, S. A. (2016). Candidates use a new teacher development process, transformative reflection, to identify and address teaching and learning problems in their work with children. Journal of Teacher Education, 67(5), 379-391. https://doi.org/10.1177/0022487116653659

Nasir, N. S., Rosebery, A. S., Warren, B., \& Lee, C. D. (2005). Learning as a cultural process: Achieving equity through diversity. In R. K. Sawyer (Ed.), Cambridge handbooks in psychology: The Cambridge handbook of the learning sciences (pp. 489-504). Cambridge, GB: Cambridge University Press. Retrieved from ProQuest ebrary

Neumeier, M. (2005). The brand gap: How to bridge the gap between business and design (Rev. ed.). Berkeley, CA: Pearson Education.

Neumeier, M. (2009). The designful company: How to build a culture of nonstop innovation. Berkeley, CA: Pearson Education.

Nguyen, N. T., \& Tangen, D. (2016). Video-stimulated recall in cross-cultural research in education: A case study in Vietnam. International Journal of Research \& Method in Education, 7288(September), 0-11. https://doi.org/10.1080/1743727X.2016.1160279

Niu, W. (2007). Western influences on Chinese educational testing. Comparative Education, 43(1), 71-91. https://doi.org/10.1080/03050060601162412

Niu, W. (2012). Confucian ideology and creativity. Journal of Creative Behavior, 46(4), 274-284. https://doi.org/10.1002/jocb.18 
Niu, W., \& Sternberg, R. J. (2006). The philosophical roots of Western and Eastern conceptions of creativity. Journal of Theoretical and Philosophical Psychology, 26(1-2), 18-38. https://doi.org/10.1037/h0091265

NZ Government. (2018). International Education Strategy 2018-2030. Retrieved from https://enz.govt.nz/assets/Uploads/International-Education-Strategy-2018-2030.pdf

O’Brien, M., Varga-Atkins, T., Umoquit, M., \& Tso, P. (2012). Cultural-historical activity theory and 'the visual' in research: Exploring the ontological consequences of the use of visual methods. International Journal of Research \& Method in Education, 35(3), 251-268. https://doi.org/10.1080/1743727X.2012.717433

Ogilvie, T., \& Liedtka, J. (2011). Designing for growth: A design thinking toolkit for managers. New York, NY: Columbia University Press.

Okada, T., \& Ishibashi, K. (2017). Imitation, inspiration, and creation: Cognitive process of creative drawing by copying others' artworks. Cognitive Science, 41(7), 18041837. https://doi.org/10.1111//ogs. 12442

Orthel, B. D. (2015). Implications of design thinking for teaching, learning, and inquiry. Journal of Interior Design, 40(3), 1-20. https://doi.org/10.1111/joid.12046

Polanyi, M. (1962). Tacit knowing: Its bearing on some problems of philosophy. Reviews of Modern Physics, 34(4), 601-616. https://doi.org/10.1103/RevModPhys.34.601

Qi, X. (2017). Reconstructing the concept of face in cultural sociology: In Goffman's footsteps, following the Chinese case. Journal of Chinese Sociology, 4(1). https://doi.org/10.1186/s40711-017-0069-y

Qin, Y., \& Te, A. Y. C. (2016). Cross-border higher education in China: How the field of research has developed. Chinese Education and Society, 49(4-5), 303-323. https://doi.org/10.1080/10611932.2016.1237851

Rauth, I., Köppen, E., Jobst, B., \& Meinel, C. (2010). Design thinking: An educational model towards creative confidence. In Proceedings of the 1st International Conference on Design Creativity, ICDC 2010. Kobe: Springer. Retrieved from https://www.designsociety.org/publication/30267

Rittel, H. W. J., \& Webber, M. M. (1973). Dilemmas in a general theory of planning. Policy Sciences, 4(2), 155-169. Retrieved from http://www.jstor.org/stable/4531523

Robinson, H. (2017). Dualism. Retrieved October 30, 2019, from https://plato.stanford.edu/entries/dualism/

Rogoff, B. (1995). Observing sociocultural activity on three planes: Participatory appropriation, guided participation, and apprenticeship. In J. Wertsch, P. Rio, \& A. Alvarez (Eds.), Sociocultural studies of the mind (Learning in doing: Social, cognitive and computational perspectives) (pp. 139-164). Cambridge: Cambridge University Press. https://doi.org/doi:10.1017/CBO9781139174299.008

Roth, W. (2009). Introduction: "Activity theory and education: An Introduction. Mind, Culture, and Activity, 11(1), 1-8. https://doi.org/10.1207/s15327884mca1101

Roth, W. M. (2007). On mediation: Toward a cultural-historical understanding. Theory \& Psychology, 17(5), 655-680. https://doi.org/10.1177/0959354307081622

Royalty, A. (2018). Design-based pedagogy: Investigating an emerging approach to teaching design to non-designers. Mechanism and Machine Theory, 125, 137-145. https://doi.org/10.1016/j.mechmachtheory.2017.12.014 
Runco, M. A. (2004). Creativity. Annual Review of Psychology, 55, 657-687. https://doi.org/10.1146/annurev.psych.55.090902.141502

Ryan, J. (2016). 'Asian' learners or 'internationalised' learners ? Taking advantage of international cultural academic flows. East Asia: An International Quarterly, 33(1), 9-24. https://doi.org/10.1007/s12140-015-9246-2

Ryan, J., \& Louie, K. (2007). False dichotomy? "Western" and "Confucian" concepts of scholarship and learning. Educational Philosophy and Theory, 39(4), 404-417. https://doi.org/10.1111/j.1469-5812.2007.00347.x

Saris, B. (2018). Crossing cultural boundaries: New Zealand visual communication design (VCD) teachers working with VCD learners in China. New Zealand Journal of Asian Studies, 20(1), 43-60.

Saris, B. (2020). A review of engagement with creativity and creative design processes for visual communication design (VCD) learning in China. International Journal of Art \& Design Education, 39(2), 306-318. https://doi.org/10.1111/jade.12262

Schön, D. A. (1984). The architectural studio as an exemplar of education for reflectionin-action. Journal of Architectural Education, 38(1), 2-9. https://doi.org/10.1080/10464883.1984.10758345

Schön, D. A. (1987). Educating the reflective practitioner. San Francisco, CA: JosseyBass.

Shung-Yu, A. F., \& Yuet-Ngor, A. L. (2001). Design education in China: New proposals to address endemic problems. Journal of Art \& Design Education, 20(2), 171-179. https://doi.org/10.1111/1468-5949.00264

Sigurðsson, G. (2017). Transformative critique: What Confucianism can contribute to contemporary education. Studies in Philosophy and Education, 36(2), 131-146. https://doi.org/10.1007/s11217-015-9502-3

Simon, H. A. (1968). The sciences of the artificial. Cambridge: MIT Press.

Siu, K. W. M., \& Contreras, G. J. (2017). Design education for fostering creativity and innovation in China. (K. W. M. Siu \& G. J. Contreras, Eds.). Hershey, PA: ICI Global.

Staats, L. K. (2011). The cultivation of creativity in the Chinese culture-past, present, and future creativity: Its meaning and means. Journal of Strategic Leadership, 3(1), 45-53.

Stake, R. E. (1995). The art of case study research. Thousand Oaks, CA: Sage.

Stake, R. E. (2006). Multiple case study analysis. New York, NY: The Guilford Press.

Stanford d.school. (2018). Design thinking bootleg. Retrieved from https://dschool.stanford.edu/resources/design-thinking-bootleg

Tan, S., \& Melles, G. (2010). An activity theory focused case study of graphic designers' tool-mediated activities during the conceptual design phase. Design Studies, 31(5), 461-478. https://doi.org/10.1016/j.destud.2010.05.002

Temple, B. (2008). Narrative analysis of written texts: Reflexivity in cross language research. Qualitative Research, 8(3), 355-365. https://doi.org/10.1177/1468794106093632

Temple, B., \& Young, A. (2004). Qualitative research and translation dilemmas. Qualitative Research, 4(2), 161-178. https://doi.org/10.1177/1468794108095079 
The Design Council. (2005). The design process: What is the double diamond? Retrieved from https://www.designcouncil.org.uk/news-opinion/design-process-what-doublediamond

Thulson, A. (2013). Contemporary practice in the elementary classroom: A study of change. Art Education, 66(1), 16-23.

https://doi.org/10.1080/00043125.2013.11519204

Tikhomirov, O. K. (1999). The theory of activity changed by information technology: Theory of creative activity. In Y. Engeström, R. Miettinen, \& R. L. Punamaki (Eds.), Perspectives on activity theory (pp. 347-352). Cambridge: Cambridge University Press.

Torrance, E. P. (1972). Torrance tests of creative thinking: Directions manual and scoring guide. Bensenville, IL: Scholastic Testing Services.

Tregloan, K. E. (2010). Looking for a-ha ... (Design epiphany). In G. Forsyth (Ed.), ConnectED 2010 - 2nd International Conference on Design Education. Sydney,

Australia: University of New South Wales. Retrieved from http://connected2010.eproceedings.com.au/papers/p49.pdf

Van Der Veer, R. (2007). Vygotski in context: 1900 - 1935. In H. Daniels, M. Cole, \& J. V. Wertsch (Eds.), The Cambridge companion to Vygotski (pp. 21-49). Cambridge.

von Thienen, J., Clancey, W., Corazza, G., \& Meinel, C. (2018). Theoretical foundations of design thinking. In H. Plattner, C. Meinel, \& L. Leifer (Eds.), Understanding innovation: Design thinking research (pp. 13-40). Cham: Springer.

Vygotsky, L. S. (1978). Mind in society: The development of higher psychological processes. In M. Cole, V. John-Steiner, S. Scribner, \& E. Souberman (Eds.), Mind in society: The development of higher psychological processes (pp. 19-119). Cambridge, MA: Harvard University Press.

Vygotsky, L. S. (2004). Imagination and creativity in childhood. Journal of Russian and East European Psychology, 42(1), 7-97. https://doi.org/10.2753/RPO10610405290173

Walker, S. (2017). Research in graphic design. Design Journal, 20(5), 549-559. https://doi.org/10.1080/14606925.2017.1347416

Wallas, G. (1926). The art of thought. London: Jonathan Cape.

Wang, S. Z. (1989). Chinese modern design: A retrospective. Design Issues, 6(1), 49-78. http://dx.doi.org/10.2307/1511577

Wang, T. (2010). A new paradigm for design studio education. International Journal of Art and Design Education, 29(2), 173-183. https://doi.org/10.1111/j.14768070.2010.01647.x

Wartofsky. (1979). Models: Representation and scientific understanding. Dordrecht, NL: Reidel.

Watkins, D. (2000). Learning and teaching: A cross-cultural perspective. School Leadership \& Management, 20(2), 161-173. Retrieved from http://search.proquest.com.helicon.vuw.ac.nz/docview/219076232?accountid=14782

Williamson, D. L., Choi, J., Charchuk, M., Rempel, G. R., Pitre, N., Breitkreuz, R., \& Kushner, K. E. (2011). Interpreter-facilitated cross-language interviews: A research 
note. Qualitative Research, 11(4), 381-394.

https://doi.org/10.1177/1468794111404319

Wilson-Mah, R., \& Thomlinson, E. (2018). Mind the gap: Chinese diploma student views of bridges and barriers to transferring into a Canadian university. Higher Education Research and Development, 37(3), 635-648. https://doi.org/10.1080/07294360.2017.1405912

Wilson, S. E., \& Zamberlan, L. (2017). Design pedagogy for an unknown future: A view from the expanding field of design scholarship and professional practice.

International Journal of Art \& Design Education, 36(1), 106-117. https://doi.org/10.1111/jade.12076

Xu, C. L., \& Montgomery, C. (2019). Educating China on the move: A typology of contemporary Chinese higher education mobilities. Review of Education, 7(3), 598627. https://doi.org/10.1002/rev3.3139

Yamagata-Lynch, L. C. (2010). Activity systems analysis methods: Understanding complex learning environments. New York: Springer.

Yang, R. (2005). Internationalisation, indigenisation and educational research in China. Australian Journal of Education, 49(1), 66-88. https://doi.org/10.1177/000494410504900104

Yang, R. (2014a). China's strategy for the internationalization of higher education: An overview. Fronters of Education in China, 9(2), 151-162. https://doi.org/10.3868/s110-003-014-0014-x

Yang, R. (2014b). Going global: Contemporary international networking in Chinese mainland universities. Chinese Education and Society, 47(1), 27-43. https://doi.org/10.2753/CED1061-1932470102

Yi, X., Hu, W., Scheithauer, H., \& Niu, W. (2013). Cultural and bilingual influences on artistic creativity performances: Comparison of German and Chinese students. Creativity Research Journal, 25(1), 97-108. https://doi.org/10.1080/10400419.2013.752260

Yuan, X., Song, D., \& He, R. (2018). Re-examining 'learning by doing': Implications from learning style migration. The Design Journal, 21(3), 313-330. https://doi.org/10.1080/14606925.2018.1444126

Zhu, H. (2015). The effectiveness of a creativity course on developing Chinese students' creative thinking (Order No. 3720939). University of the Pacific. Stockton, CA. Retrieved from http://search.proquest.com.helicon.vuw.ac.nz/docview/1726028695? accountid $=14782$ 
Appendices

Appendix 4.1. Information sheet and consent form to video and interview students and teachers

\title{
Branding Project: A China tea house
}

NB: Since a single Chinese character accounts for a single word, the information sheet and consent form to video and interview students for the Miao illustration project: Playing cards, and the teachers' semi-structured interviews has not been replicated. The information is identical, only the dates were adjusted in both instances. For the Miao illustration project the final date for withdrawal was 10 November 2018, and for the teachers interviews, the final date for withdrawal was 10 May 2019.

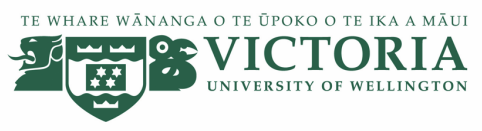

\author{
Examining Chinese visual communication design learners' \\ creative design processes in higher education institutions \\ 考察在高等教育院校中中国视觉传播设计学习者的创意设计过程 \\ INFORMATION SHEET FOR STUDENTS for Video and Interviews \\ 视频和访谈学生信息表
}

\begin{abstract}
This is an invitation to take part in some research. Please read this information before deciding whether or not to take part. If you decide to participate, thank you. If you decide not to take part, thank you for considering my request. Please note that participation or non-participation will not compromise your grades for this paper, as the other NZ teacher will be marking course work from this class. 这是参加一些研究的邀请。请先阅读此信息, 然后再决定是否参加。如果你决定参 加, 谢谢。如果你决定不参加, 也感谢你考虑我的请求。请注意, 参加或不参与都不会影响本 课程的成绩，因为其他新西兰教师将在批改本课程的课程作业。
\end{abstract}

\section{Who am I? 我是睡?}

As you know my name is Brenda Saris and I am a teacher at [Higher education institution name removed]. I am doing some research towards my PhD at Victoria University in Wellington. 如你知 道我的名字是Brenda Saris, 我是新西兰理工学院的老师。为取得惠灵顿维多利亚大学博士学 位, 我正在做一些课题项目研究。

\section{What is the aim of the project? 研究项目目的是什么?}

The principle aim of this research is to better understand Chinese students' learning needs and design processes, so effective strategies may be developed to enhance the pedagogical experience for both learners and teachers in higher education institutions. This research has been approved by the Victoria University of Wellington Human Ethics Committee [Approval number 24689] and the [Higher education institution name removed] Research and Ethics Committee. 这项研究的主要目的 
是更好地了解中国学生的学习需求和设计过程, 因此可以制定有效的策略来提高在高等教育学 院中学习者和教师的教学经验。这项研究已获得惠灵顿维多利亚大学人类伦理委员会批准 [批 准号 24689]

\section{How can you help? 你怎么帮忙?}

If you agree to take part there will be two parts to the research. First, I will video you at Hunan City University, School of Art and Design along with three other participants while you are in class completing a design task. The video recording will take four hours in class time. Second, I will interview you together with the partner that you worked with. I will ask you questions about your creative design processes. The interview will take between 30 minutes to one hour and we will decide on a convenient place and time to do this. I will audio record the interview with your permission and write it up later. You can choose to not answer any question or stop the interview at any time, without giving a reason. You can withdraw from the study by contacting me at any time before 10 March 2018. If you withdraw, the information you provided will be destroyed or returned to you. 如果你同意参与, 研究将分两部分。首先, 我将在录制你在湖南城市大学, 艺术设计 学院及和其他三位参与者一起, 在课堂上同时完成一个设计任务。视频录制将需要 4 个小时 的上课时间。第二, 我采访你和你的一起工作搭档。我会问你们有关创意设计过程的问题。采 访将需要 30 分钟到 1 小时, 我们会约定一个方便的地方和时间来做。经你许可我将录音采 访, 并晚点写下来。你可以随时选择不回答任何问题或随时停止采访, 而不用给出一个理由。 你可以在 2018 年 3 月 10 日之前随时与我取得联系撤回。如果你撤回, 你提供的信息将被销 毁或退回给你。

What will happen to the information you give? 你提供的信息会发生什么?

This research is confidential. This means that the researchers named below will be aware of your identity but the research data will be aggregated and your identity will not be disclosed in any reports, presentations, or public documentation. Only my supervisors, three other New Zealand staff who teach you at HCU and I will read the notes or transcript of the interview. The interview transcripts, summaries (which will be in English) and any recordings will be kept securely and destroyed 10 years after the research ends. 这项研究是保密的。这意味着下面提到的研究人员 将会了解你的身份, 但研究数据将被汇总, 你的身份不会在任何报告里、演示文稿或公开文档 中被披露。只有我的主管导师, 另外三名在湖南城市学院教你们的教师, 我将阅读采访的笔记 或记录。研究结束后采访记录, 摘要 (英文) 和任何录音将安全保留, 10 年后, 将被销毁。

\section{What will the project produce? 此项目将会产生什么?}

The information from my research will be used in my PhD dissertation AND for submissions to academic publications and conferences. 我的研究信息将用于我的博士论文, 并提交给学术期刊 和研讨会议使用。

If you accept this invitation, what are your rights as a research participant? 如果你接受此邀请, 你作为研究参与者的权利是什么?

You do not have to accept this invitation if you don't want to. 如果你不想, 你不必接受此邀请。 
If you do decide to participate, you have the right to: 如果你决定参加, 你有的权力:

- ask for the video to be turned off at any time during the recording.

- choose not to answer any question;

- ask for the recorder to be turned off at any time during the interview;

- withdraw from the study before [10 March 2018];

- ask any questions about the study at any time;

- receive a copy of your interview recording;

- receive a copy of your interview transcript;

- read over and comment on a written summary of your interview;

- be able to read any reports of this research by emailing the researcher to request a copy.

- 在录制过程中随时要求关闭视频;

- 选择不回答任何问题;

- 要求录音机在采访过程中随时关闭;

- 在[2018 年 3 月 10 日]之前撤出此参与研究;

- 随时询问有关研究的任何问题;

- 收到你的采访录制副本;

- 收到你的采访记录副本;

- 阅读你采访的书面摘要并提出意见评论;

- 可以通过电子邮件联系研究人员来要求获得一个副本并阅读本研究的任何报告。

If you have any questions or problems, who can you contact?

如果你有任何问题或疑问, 你可以联系谁?

If you have any questions, either now or in the future, please feel free to contact [either/me]: 如果你有任何问题, 现在或将来, 请随时联系[任何一个/我]:

Student: 学生:

Name: 姓名: Brenda Saris

Brenda.saris@vuw.ac.nz

大学邮箱地址:

Brenda.saris@vuw.ac.nz
Supervisors（导师）School of Education: 教育学院: Name: 姓名: Dr Stephanie Doyle

Stephanie.doyle@vuw.ac.nz / 电话 : +64 44636657

Name: 姓名: Dr Judith Loveridge

Judith.loveridge@vuw.ac.nz / 电话 : +64 44636028

Human Ethics Committee information. 人类伦理委员会信息。

If you have any concerns about the ethical conduct of the research you may contact the Victoria University HEC Convenor: Associate Professor Susan Corbett. Email susan.corbett@vuw.ac.nz or telephone +64-4-463 5480. 如果你对研究的道德行为有任何疑问, 可以联系维多利亚大学 HEC 召集人：副教授 Susan Corbett 电邮 susan.corbett@vuw.ac.nz 或致电+64-4-463 5480。 


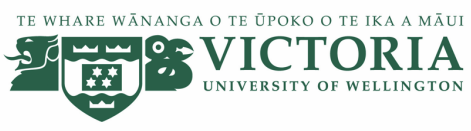

Examining Chinese visual communication design learners' creative design processes in higher education institutions

\section{考察在高等教育学院中中国视觉传播设计学习者的创意设计过程}

\section{CONSENT TO VIDEO and INTERVIEW}

视频和采访同意书

This consent form will be held for 10 years. 本同意书将持有 10 年。

Researcher: Brenda Saris. School of Education Victoria University of Wellington. 研究员：惠灵顿维多利亚大学教育学院 Brenda Saris。

- I have read the Information Sheet and the project has been explained to me. My questions have been answered to my satisfaction. I understand that I can ask further questions at any time.

- 我已阅读了信息表, 并向我解释了该项目。我的问题解答使我满意。我明白我可以随时问 更多的问题。

- I agree to take part in a video recording of a design class (four hours) and an audio recorded interview (between 30 minutes and one hour).

- 我同意参加设计课（四小时）的视频录制和录音采访（30 分钟到一小时）。

- I understand that: 我明白 :

- I may withdraw from this study at any point before 10 March 2018 , and any information that I have provided will be returned to me or destroyed.

- 我可以在 2018 年 3 月 10 日之前的任何时候退出本研究，我提供的任何信息将退回给我或 被销毁。

- The information I have provided will be destroyed 10 years after the research is finished.

- 我提供的信息将在研究完成 10 年后销毁。

- Any information I provide will be kept confidential to the researcher the transcriber/translator and the supervisor

- 我提供给研究人员, 誊写/译者和导师的任何信息将被保密

- I understand that the results will be used for a PhD dissertation AND for submissions to academic publications and conferences, which may include open online web platforms

- 我了解结果将用于博士论文, 并提交给学术期刊和学术研讨会议使用, 其中可能包括开放 的在线网络平台。

- My name will not be used, nor will any information that would identify me.

- 我的名字不会被使用, 也不会有任何信息可以识别出我。 
1. I would like a copy of the recording of my interview:

我想要一个我的采访的录制副本

Yes $\square \quad$ No $\square$

2. I would like a copy of the interview transcript:

我想要一个采访脚本的副本:

Yes $\square \quad$ No $\square$

3. I would like a summary of my interview which will be in English: 我想要一份采访的英文摘要

Yes $\square \quad$ No $\square$

4. I would like to see an electronic link to the final thesis and have added my email address below.

我想看到最后的论文一个电子链接, 并在下面留下我的

电子邮件地址

Yes $\square \quad$ No $\square$

Signature of participant:

参与者签名

Name of participant:

参与者姓名：

Date:

日期：

Contact details:

联系方式 : 


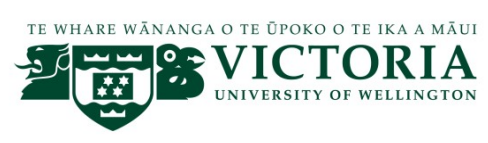

\section{Translating confidentiality agreement}

\section{Examining Chinese visual communication design learners' creative design processes in higher education institutions}

Principal Investigator: Brenda Saris

I, agree to ensure that the [student and teacher interviews] I translate will remain confidential to the researcher and her supervisors: Dr. Stephanie Doyle and Dr. Judith Loveridge.

I agree to take the following precautions:

1. I will ensure that no person, other than Brenda Saris, Dr. Stephanie Doyle and Dr. Judith Loveridge, hears or sees the recording.

2. I will ensure that no other person has access to my computer/device.

3. I will delete the files from my computer/device once the transcription and translation has been completed.

4. I will not discuss any aspect of the recording with anyone except Brenda Saris.

Signature:

Date: 
Appendix 4.3. Summary of participant ethical informed consents

\begin{tabular}{|c|c|c|c|c|}
\hline & $\begin{array}{l}\text { Consent to video } \\
\text { and interview }\end{array}$ & $\begin{array}{l}\text { Consent to } \\
\text { interview }\end{array}$ & $\begin{array}{l}\text { Transcribing } \\
\text { and translating } \\
\text { confidentiality } \\
\text { agreement }\end{array}$ & $\begin{array}{l}\text { Withdrawal } \\
\text { date }\end{array}$ \\
\hline $\begin{array}{l}\text { Branding project } \\
\text { (case study } 1 \text { ) }\end{array}$ & $\begin{array}{l}8 \text { participants } \\
7 \text { September } 2017\end{array}$ & N/A & $\begin{array}{l}1 \text { interpreter } \\
11 \text { September } 2017\end{array}$ & 10 March 2018 \\
\hline $\begin{array}{l}\text { Miao illustration } \\
\text { project } \\
\text { (case study 2) }\end{array}$ & $\begin{array}{l}8 \text { participants } \\
29 \text { May } 2018\end{array}$ & N/A & $\begin{array}{l}1 \text { interpreter } \\
4 \text { June } 2018\end{array}$ & 10 November 2018 \\
\hline $\begin{array}{l}\text { Teachers' } \\
\text { interviews }\end{array}$ & N/A & $\begin{array}{l}8 \text { participants } \\
13-27 \text { September } \\
2018\end{array}$ & $\begin{array}{l}2 \text { interpreters } \\
19 \text { September } 2018\end{array}$ & 10 May 2019 \\
\hline
\end{tabular}




\section{Appendix 4.4. Examples of written reflections}

\section{Summarize the lesson.}

In this course, I have learned a lot of new knowledge and gained more

ideas.

At first I decided that a sign didn't want to change. But then I tried a variety of other designs, and I got a lot of surprises.I realized that should be brave to try many ways when designing.

After the computer processing, the pattern gave me more ideas, which led me to learn how to combine hand painting and design with a computer

When making products, I found that not all good logos fit on the product, so consider this too.

In the end, I'm going to try and be brave when I'm designing, and I'm going to look at other designs, hope I can make better designs.

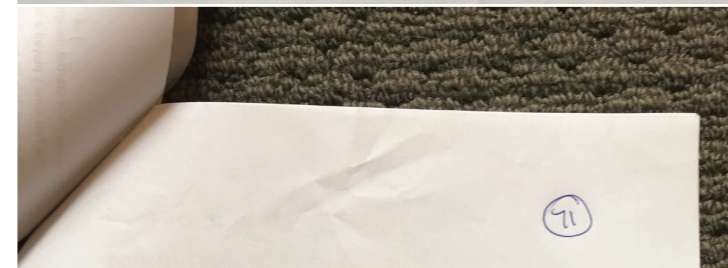

With regard to logo design, I learned a lot in the process. I know other uses of Al. This time we are going to design a logo around "tea one". In the design process, I know, to do a thing, we must try various ways to show. Try to make a few more options for yourself to run for a good one. For example, use different colors, different font sizes, different placement, etc. to adjust. In this process, if you have difficulties, you need to be patient and solve them step by step. When you can't, stand up, walk, relax, or ask the other students for advice. Sometimes I have a lot to do with it, and it gets complicated. In fact, simple things are also good:

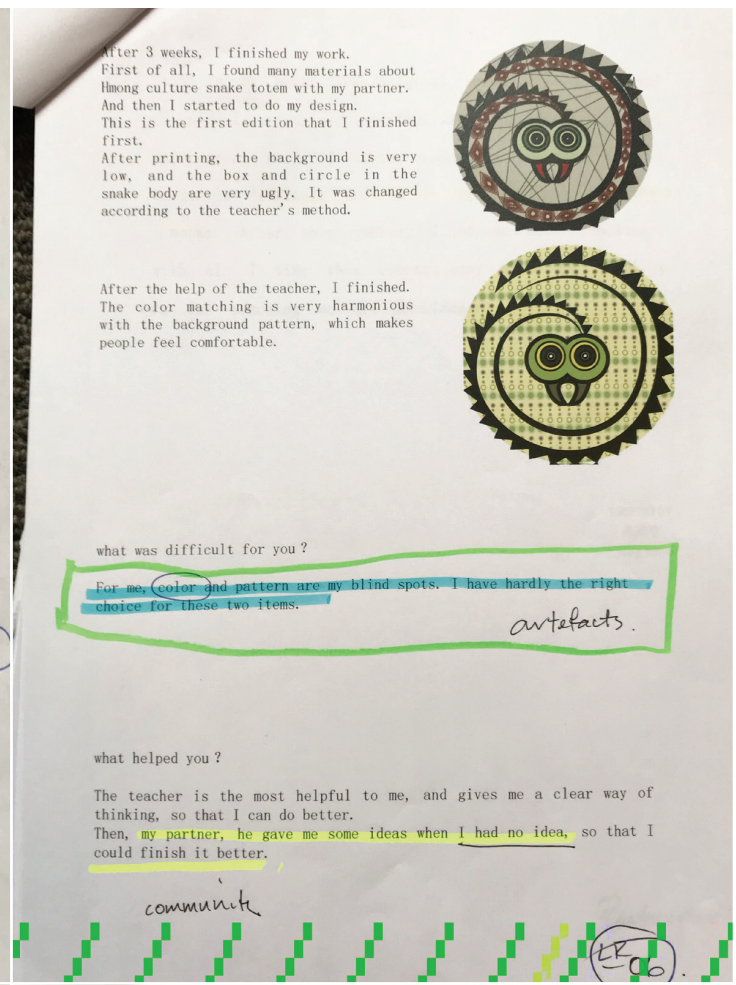

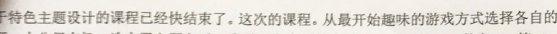
道, 十分得有起, 选定了主题之后。我需要考虑到的事情有 3 个。第一。数字 6 。第二。

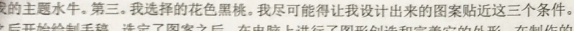
之后开始经制手䅧。选定了图案之后: 在电脑上进行了图形创造和完善它的外形。在制作的 中途。我们还选行了一系列的花色创造。这些制作起来的时候都并不是很困难。但是在制作

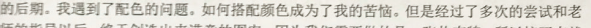

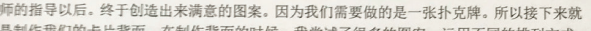
定制作我们的卡片背面。在制作棐面的时傺。我尝试了很多的图案, 运用不同的排列方式。

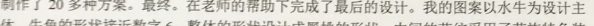

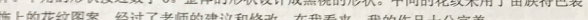

The course on theme design is almost over. This is the course. Choose the theme from the beginning of the fun game. It's very interesting, and after the theme is selected. There are 3 things As far as I can, I want my design to be close to these three conditions. Then begin to draw the manuscript. After the pattern was selected. Graphics are created on the computer ind tit shepe is improved. In the midway of production. We have also made a series of color creation. These are not very difficult to make. But at the end of the production. I have a problem with color matching. How to match the color has become my distress. But after many attempts and teachers' guidance, Finally created a satisfactory pattem. Because what we need to do is a poker card. So the next step is to make the back of our card. When making the back. I tried a lot of patterns, using different arrangements. More than 20 schemes have been made. Final. The final design was completed with the help of the teacher. My design is based on the design of buffalo. The shape of the horns is close to the number 6 . The shape of the whole is designed as a spade. The patterns in the middle are decorated with decorative pattems on the Miao nationality. After the teacher's advice and revision. In my opinion。 My work is perfect. 
The three-week course taught me a lot to realize. From the beginning to determine the design theme is a very interesting way. After understanding the clear direction, he began to gradually draw a series of drawings, referenced a lot of design elements and materials during the period, and also thought about finishing a number of substantive ideas and ideas about the Miao elements. The teacher gave me a lot of opinions, letting me abandon some traditional traits, make some bold attempts and changes, and gave me a lot of very useful inspiration and ideas in the sketching stage. When we started drawing the main image, we taught us to use shapes to cut the figure. This is a bold experiment and interesting skill. Let us not only use the previous method to realize our own design concept, but use different methods to help. We have made bold attempts. This is a breakthrough for us in the design industry. The idea of the pattern of the main figure is an annoyance to me. There are also some controls on the colors that have caused me to encounter great difficulties in the later learning. The teacher also gave me some

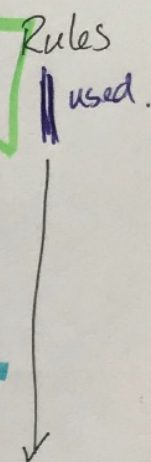
suggestions and help me to make breakthroughs in some ideas. The requirement of the pattern can express the meaning of the theme, embellish the subject without losing the integrity and color of the subject. Let me try some shallow colors, and at the same time use dark colors in some places to make a strong contrast. Although the process is rather frustrating; I still finished my own work. At the same time, the last typesetting classmate also gave me some help. These are the most valuable experiences I feel in the course.

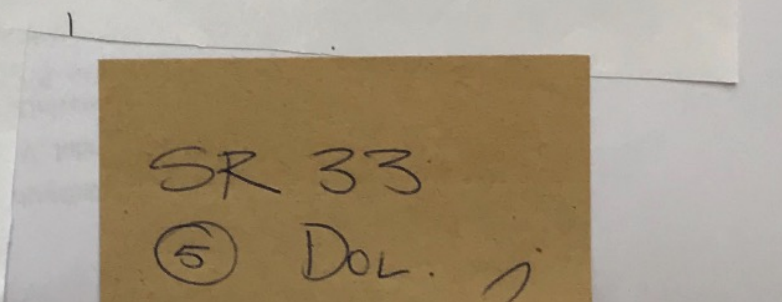


Appendix 6.1. Branding Project: A China tea house

\begin{tabular}{|c|c|c|c|}
\hline $\begin{array}{l}\text { Class } \\
\text { and date } \\
(2017)\end{array}$ & Design thinking phase & $\begin{array}{l}\text { Activity modes: } \mathrm{T}=\text { Thinking, } \\
\mathrm{E}=\text { Examining, } \mathrm{N}=\text { Notes, } \\
\mathrm{S}=\text { Sketching, } \mathrm{C}=\text { Computer. }\end{array}$ & $\begin{array}{l}\text { Data gathering / video } \\
\text { capture periods }\end{array}$ \\
\hline $\begin{array}{l}\text { 1. Wednesday } \\
6 \text { Sept. }\end{array}$ & $\begin{array}{l}\text { Empathise/define } \\
\text { Problem formation, concept } \\
\text { creation } \\
\text { - Problem structuring }\end{array}$ & $\begin{array}{l}\mathbf{T}, \mathbf{N}, \mathbf{S} \\
\text { Dyad formation }\end{array}$ & \\
\hline $\begin{array}{l}\text { 2. Thursday } \\
7 \text { Sept. }\end{array}$ & $\begin{array}{l}\text { Ideate } \\
\text { - Idea generating process }\end{array}$ & $\begin{array}{l}\mathrm{T}, \mathrm{E}, \mathbf{N}, \mathbf{S} \\
\text { Individual activity }\end{array}$ & \\
\hline $\begin{array}{l}\text { 3. Friday } \\
8 \text { Sept. }\end{array}$ & $\begin{array}{l}\text { Ideate } \\
\text { - Idea generating process }\end{array}$ & $\begin{array}{l}\text { T, E, N, S } \\
\text { 1. Mindmap to locate key } \\
\text { concepts (brand values) } \\
\text { 2. Thumbnail sketches } \\
\text { to explore concepts } \\
\text { Pictorial: } \\
\text { Visualising brand values } \\
\text { Typographic: } \\
\text { Combining Mandarin } \\
\text { characters and English } \\
\text { letterforms } \\
\text { Dyad activities }\end{array}$ & $\begin{array}{l}\text { 1. Mindmap ( } 30 \text { minutes) } \\
\text { 2. Thumbnail sketches } \\
\text { Pictorial ( } 1 \text { hour) } \\
\text { Typographic ( } 1 \text { hour) }\end{array}$ \\
\hline $\begin{array}{l}\text { 4. Tuesday } \\
12 \text { Sept. }\end{array}$ & $\begin{array}{l}\text { Technical, Illustrator } \\
\text { exercises }\end{array}$ & Individual activity & \\
\hline $\begin{array}{l}\text { 5. Wednesday } \\
13 \text { Sept. }\end{array}$ & $\begin{array}{l}\text { Ideate } \\
\text { Solution generation } \\
\text { - Iterative process, } \\
\text { preliminary computer work }\end{array}$ & $\begin{array}{l}\mathbf{T}, \mathbf{E}, \mathbf{S}, \mathbf{C} \\
\text { Dyad activity }\end{array}$ & \\
\hline $\begin{array}{l}\text { 6. Thursday } \\
14 \text { Sept. } \\
\text { 7. Friday } \\
15 \text { Sept. }\end{array}$ & $\begin{array}{l}\text { Prototype } \\
\text { Evolution and evaluation } \\
\text { - Iterative process, transition } \\
\text { to synthesis }\end{array}$ & $\begin{array}{l}\text { T, E, S, C } \\
\text { Dyad and individual activity }\end{array}$ & $\begin{array}{l}\text { Preliminary designs: } \\
\text { Iterative computer } \\
\text { experiments ( } 1.5 \text { hours) }\end{array}$ \\
\hline $\begin{array}{l}\text { 8. Tuesday } \\
19 \text { Sept. } \\
\text { 9. Wednesday } \\
20 \text { Sept. } \\
\text { 10. Thursday } \\
21 \text { Sept. }\end{array}$ & $\begin{array}{l}\text { Prototype / test (evaluate) } \\
\text { Evolution and evaluation } \\
\text { - Iterative process, transition } \\
\text { to synthesis and refinements }\end{array}$ & $\begin{array}{l}\mathrm{T}, \mathrm{E}, \mathrm{S}, \mathrm{C} \\
\text { Dyad and individual activity }\end{array}$ & \\
\hline $\begin{array}{l}\text { 11. Friday } \\
22 \text { Sept. } \\
\text { 12. Tuesday } \\
26 \text { Sept. }\end{array}$ & $\begin{array}{l}\text { Test (evaluate) } \\
\text { - Iterative process, design } \\
\text { refinements }\end{array}$ & $\begin{array}{l}\text { T, E, C } \\
\text { Dyad and individual activity }\end{array}$ & \\
\hline
\end{tabular}

The following 'slides' are a summary of information and instruction conveyed to the students in the twelve classes, and photographic evidence of the learning. 
(Source: Unless indicated, all are author images of VCD student work at HNCU).

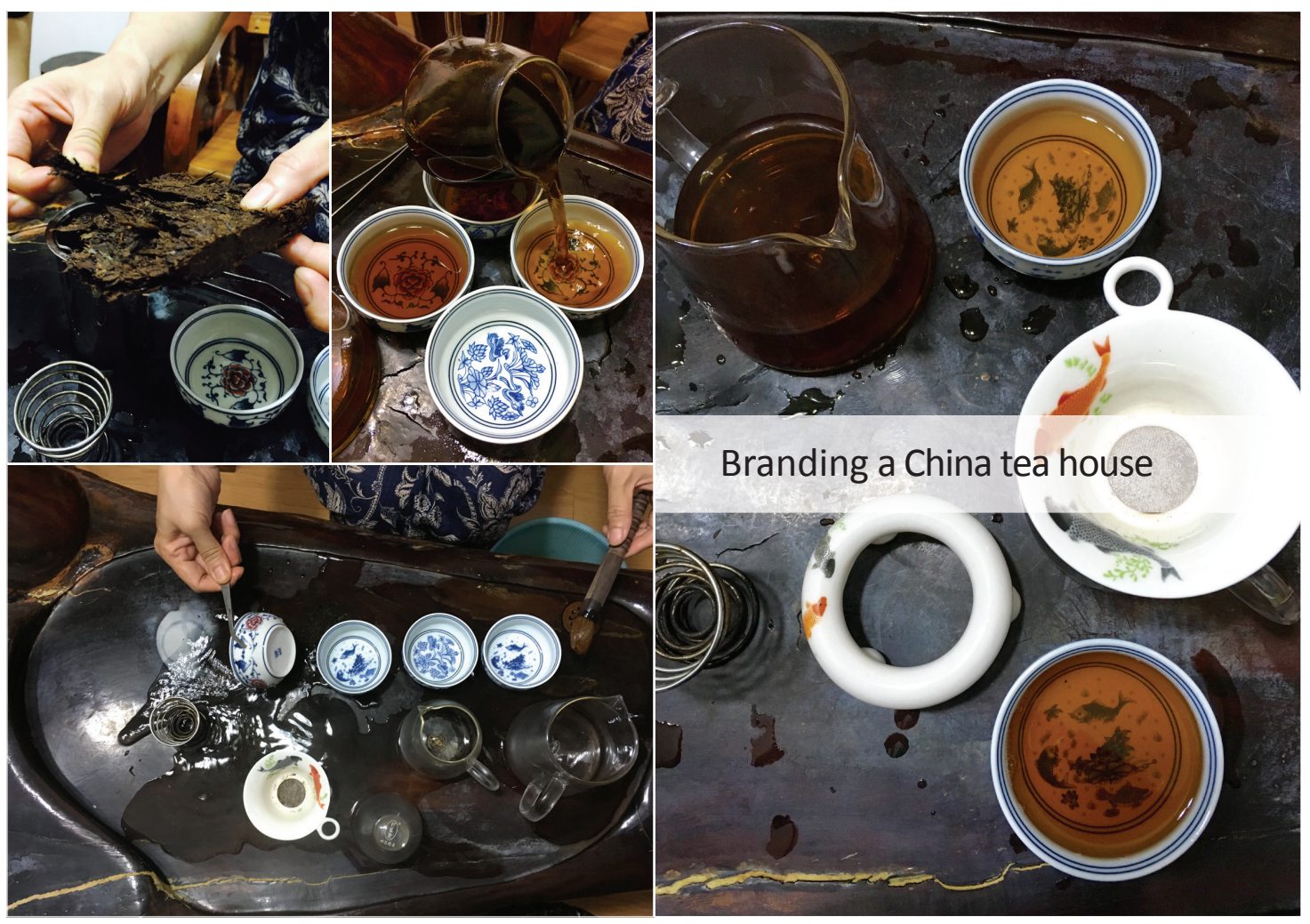

Source: Friend conducting a tea ceremony in a private residence in Yiyang (author images).

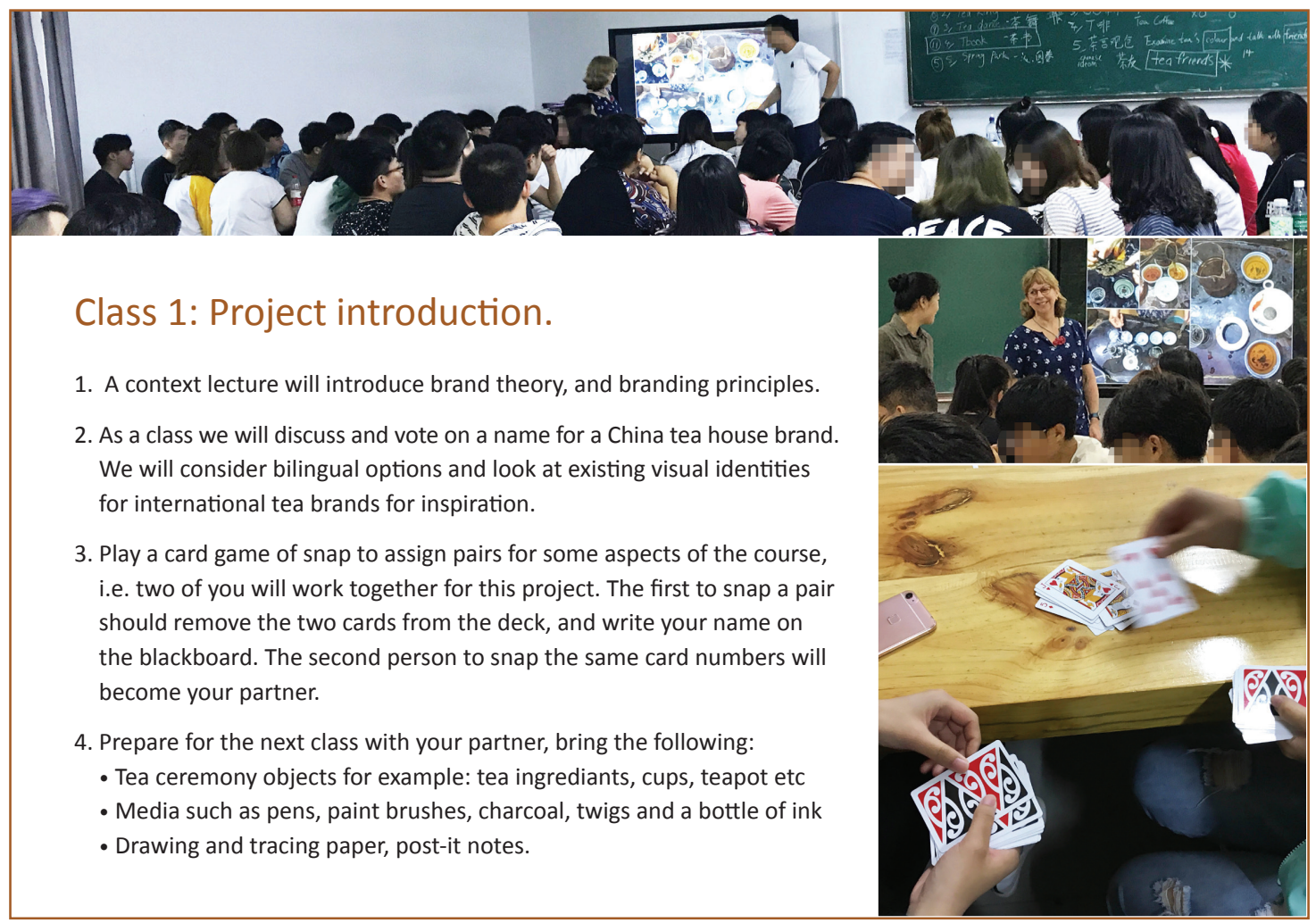




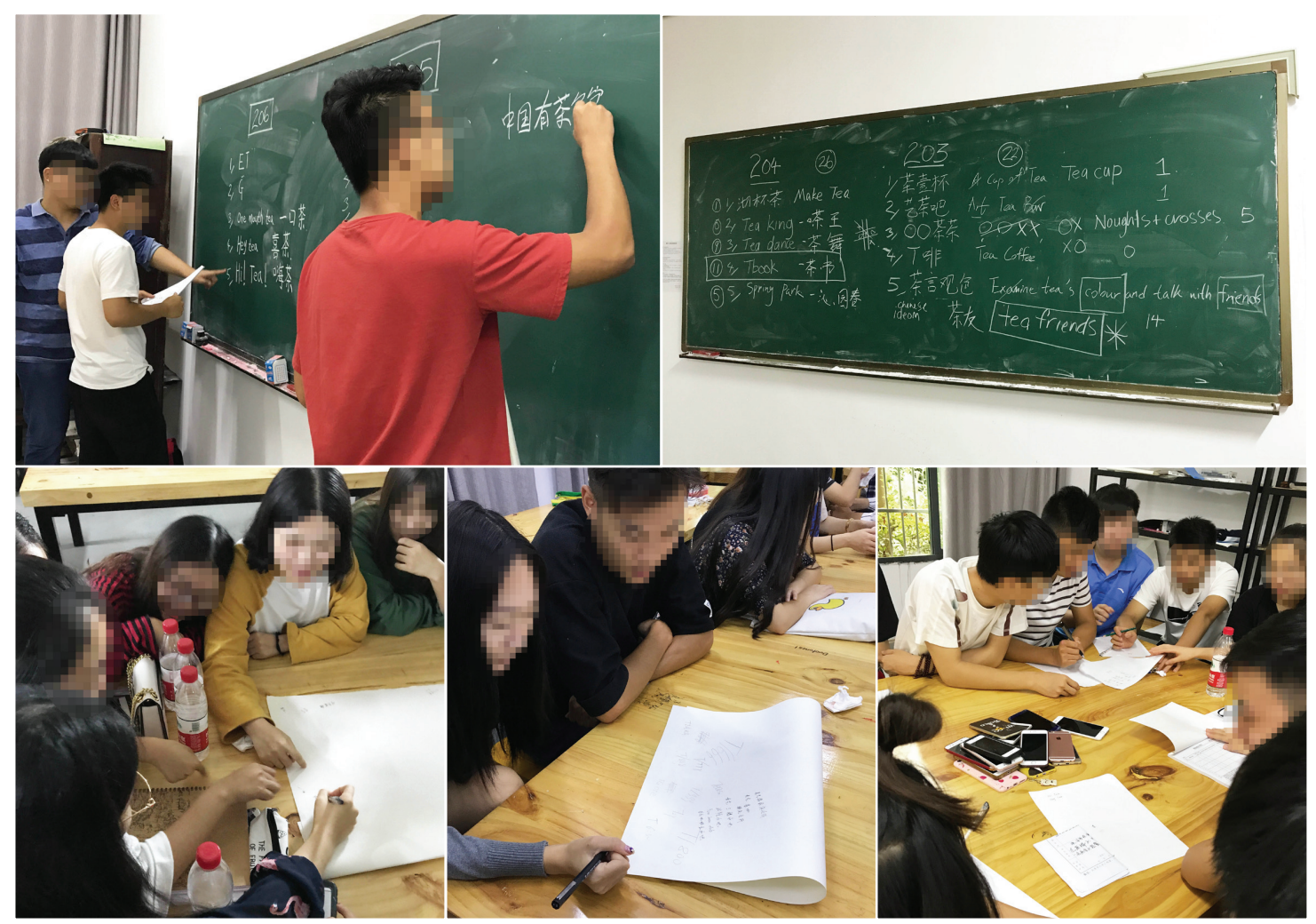

Class 2: Observational transformation / drawing exercises.

In VCD every line, shape, colour, typeface and combination helps to construct meaning.

You will begin this project with observational drawing. These drawings will be developed further through a process of stylisation. The focus is on generating an extensive range and variety of drawings.

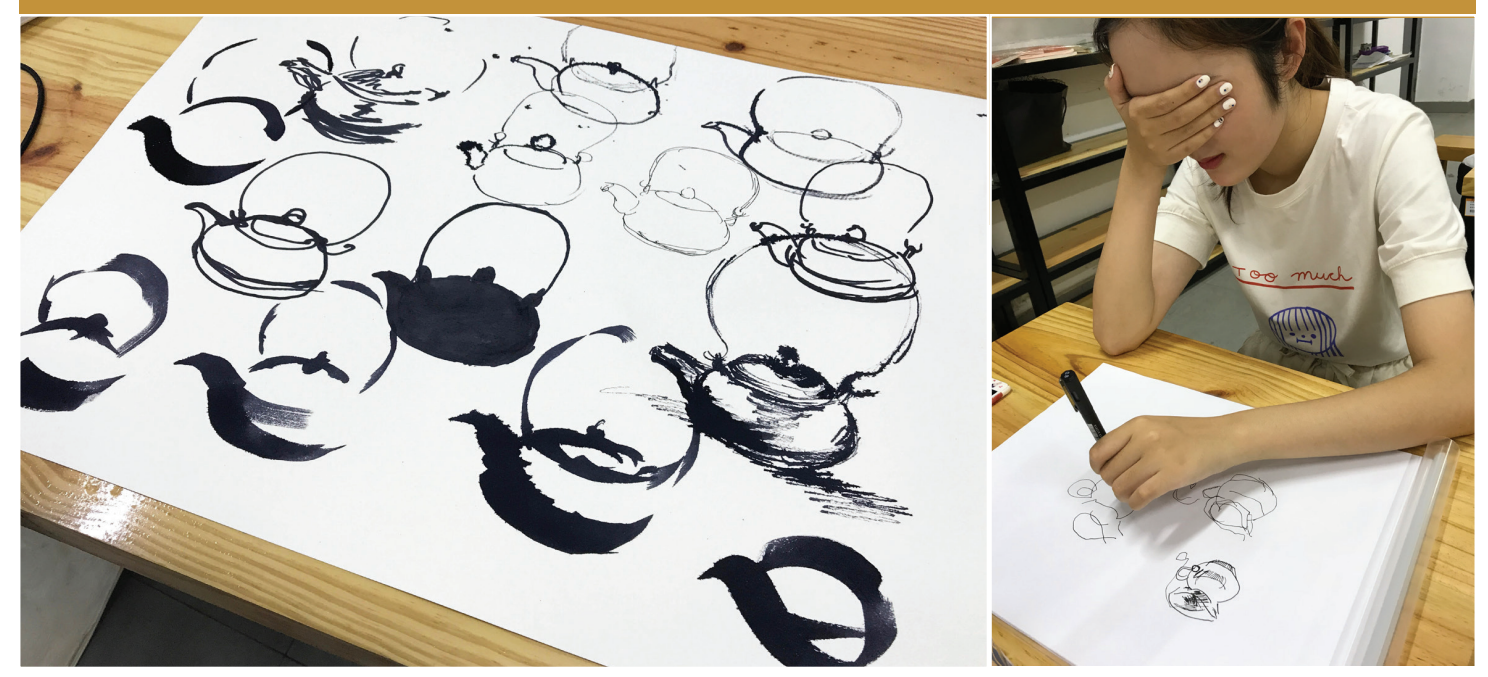




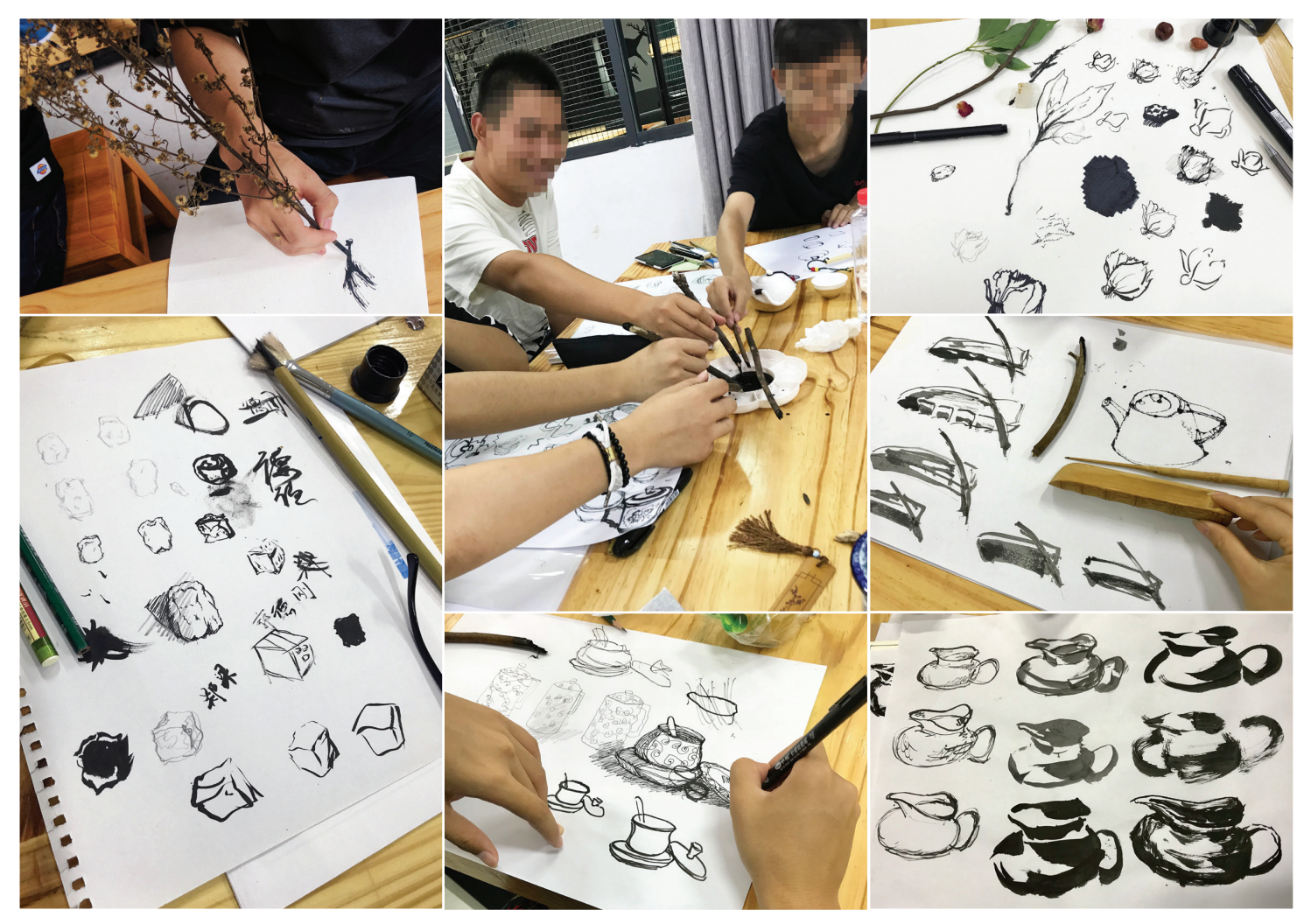

Class 3: Designing meaning / working in pairs.

1. MINDMAP (Brainstorming on post it notes).

- Using key words (may involve personal interpretation), describe the qualities of a tea ceremony in China.Include Chinese and English translations. Example starter words may include: community, informal, friendly, strong, tasty, earthy, sweet, etc.

The purpose of this exercise is to determine a brand value (essence) to help establish a design concept. The aim is to find a word which acts like a signpost.
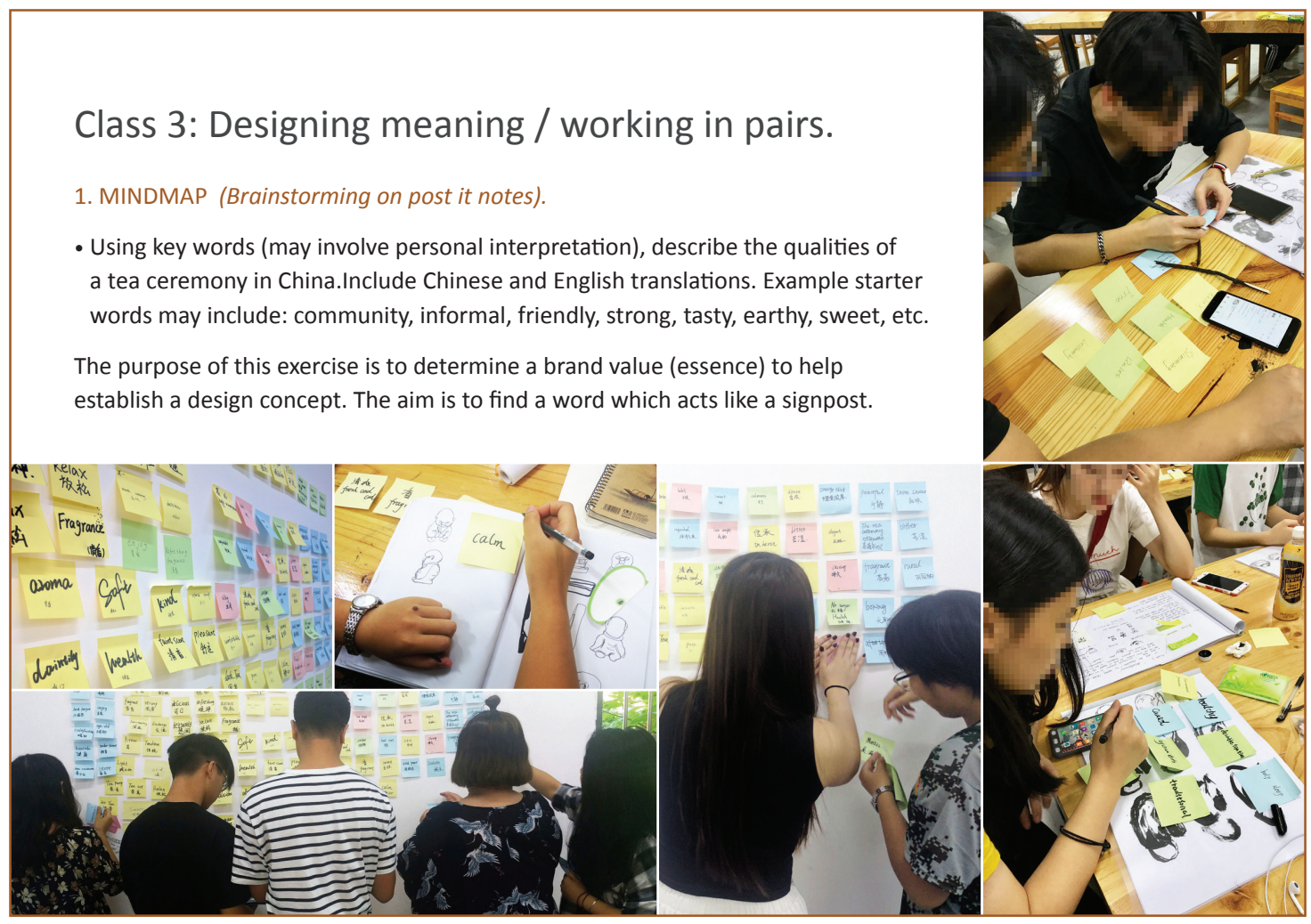
2. THUMBNAIL SKETCHES (Pictorial).

Explore and visualise concepts, sketching.

- Make quick sketches which combine the drawings from the last class and the first English letter of your brand name. Consider the words from your mind-mapping. How can the feeling of the words reflect or express new meaning?

This example combines the letter $T$ with a bird and a wave. This student considered words which included: protection, fragile and camouflage.
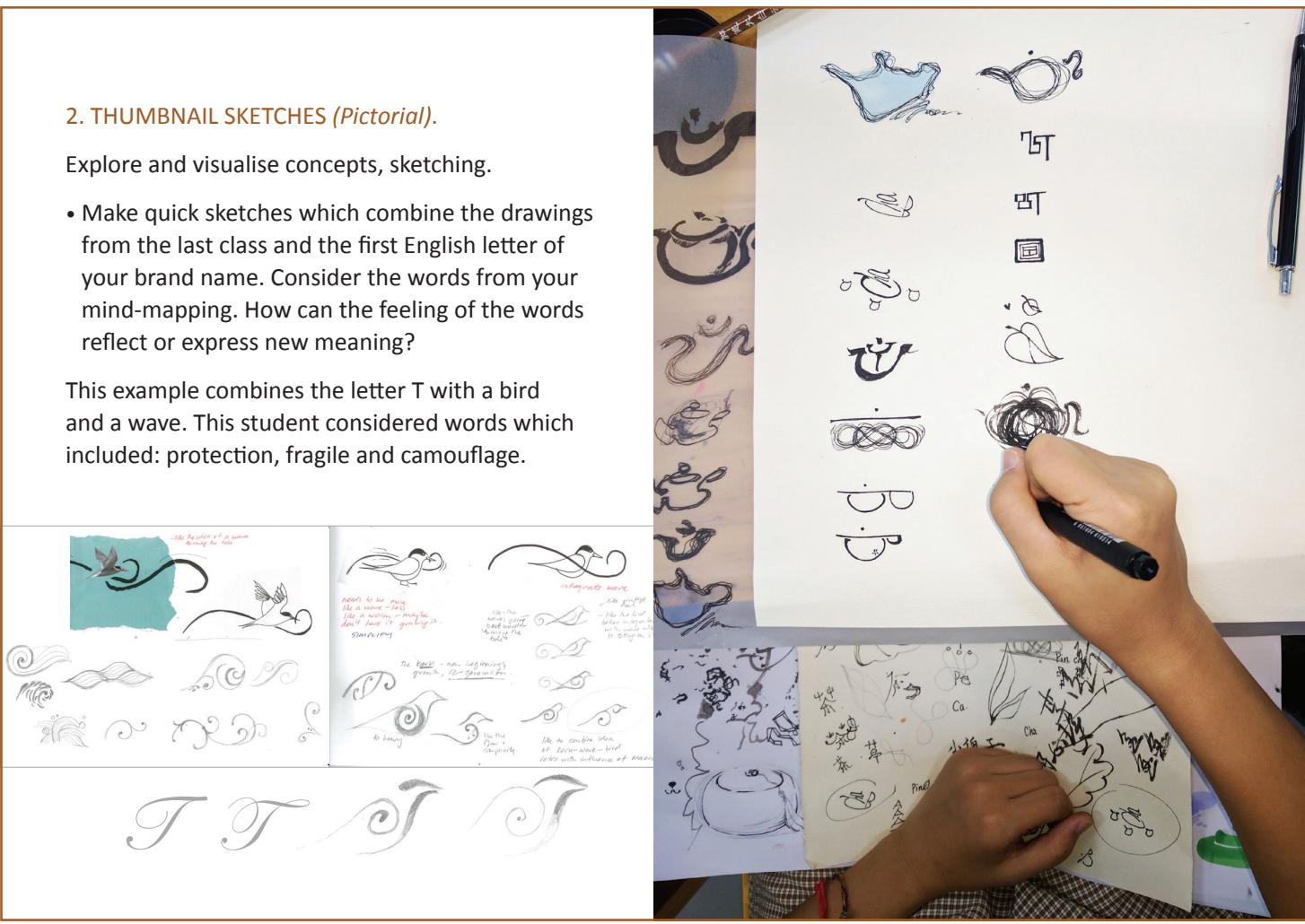

2. THUMBNAIL SKETCHES (Typographic).

Explore and visualise concepts, sketching.

- Make quick sketches which combine both Chinese characters, and English letterforms. Experiment with your brand name and your selected 'essence' words. Take care to retain legibility and meaning.

The example below, combined the English word 'book' and the Chinese 'book' character in a logo design for a bilingual bookstore.
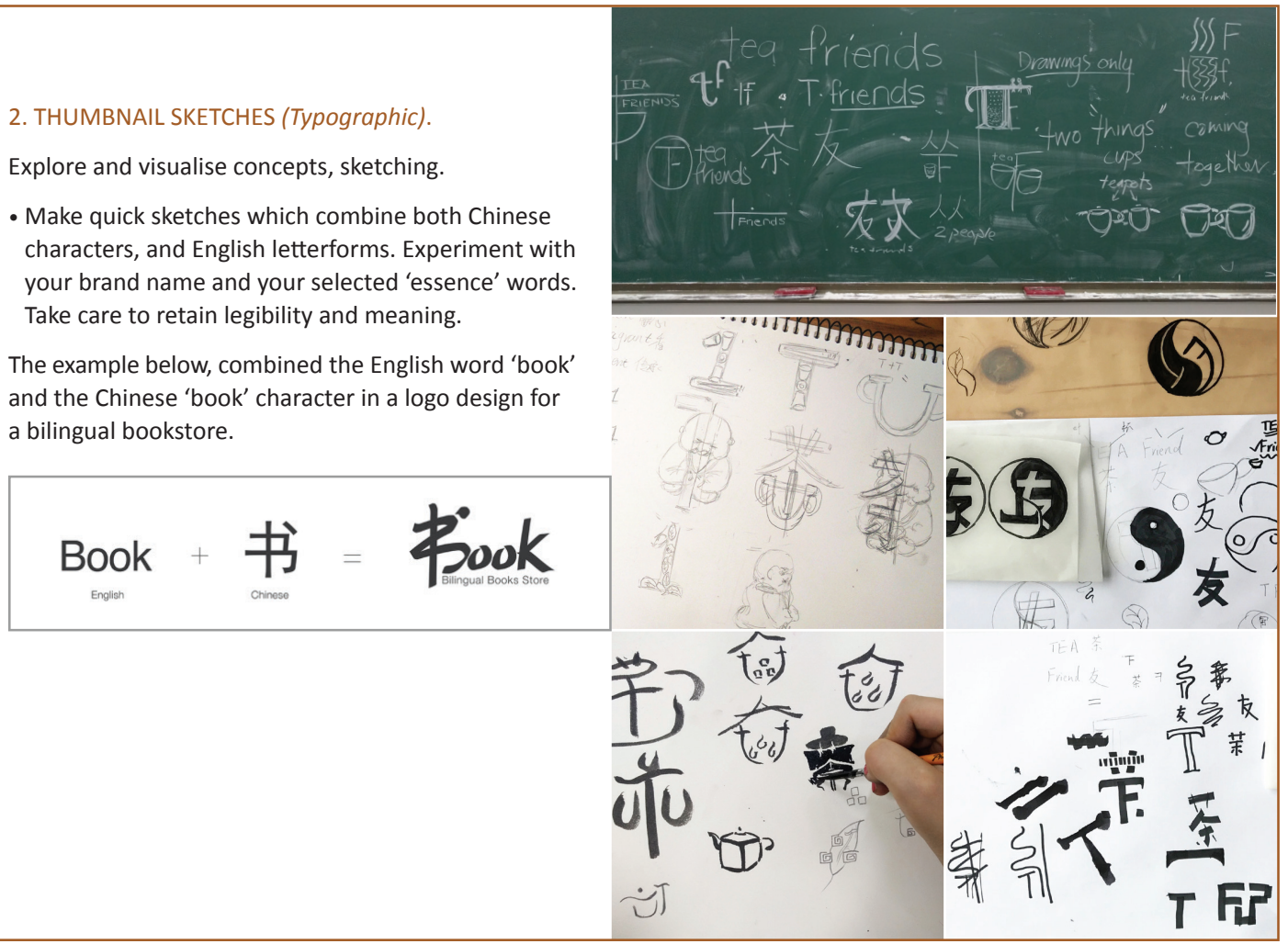

Source: Project exemplar image retrieved from https:/www.behance.net/gallery/42405633/Bilingual-Books-Store 
Class 4: Technical, Illustrator exercises.

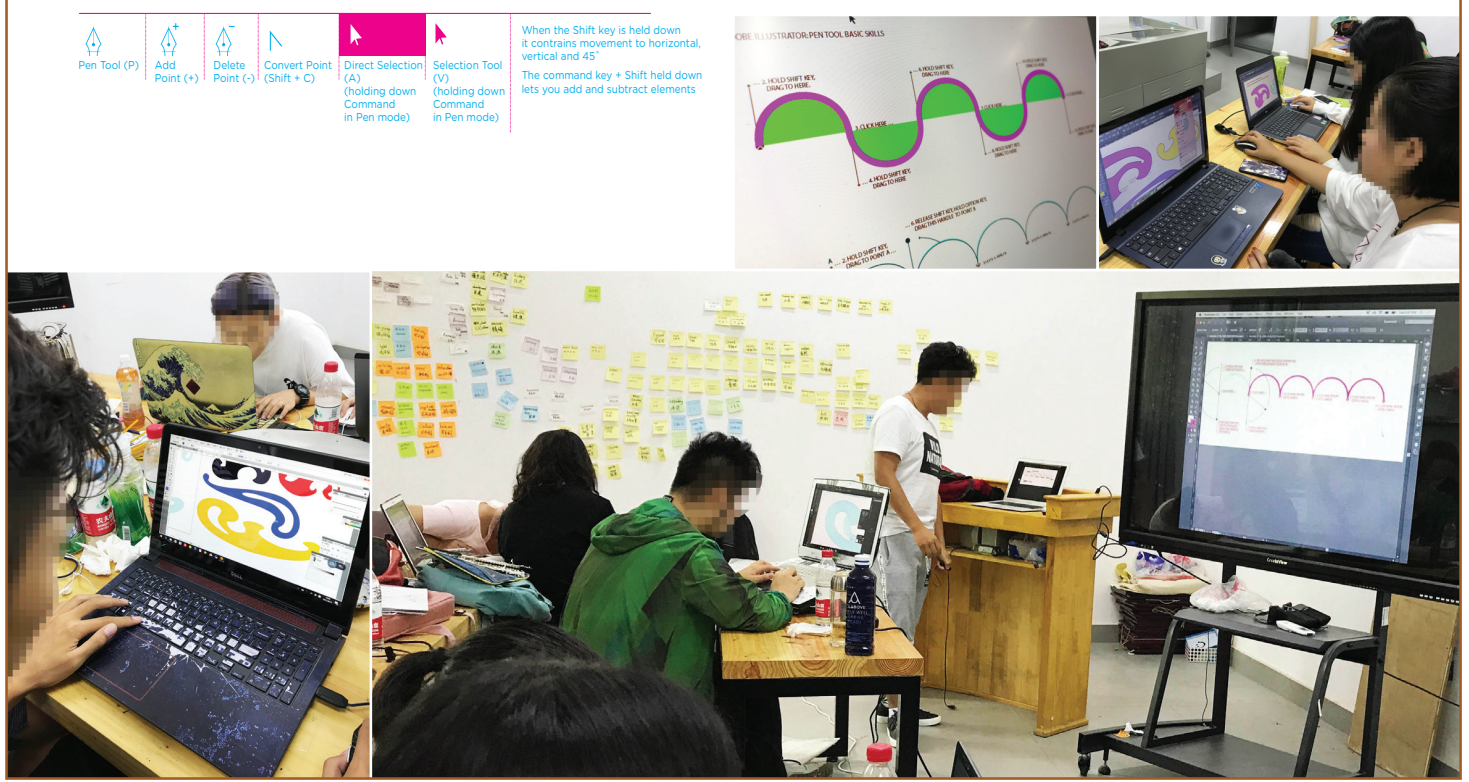

Class 5: Analogue to digital / logo design / working in pairs.

- Using Illustrator techniques from yesterday, start to explore logo design options with your computer. Try to design as many different alternatives (iterations) that you can for comparison and further design development.

This colour palette has been selected from various images in this presentation. You may use this colour palette or choose your own colours.
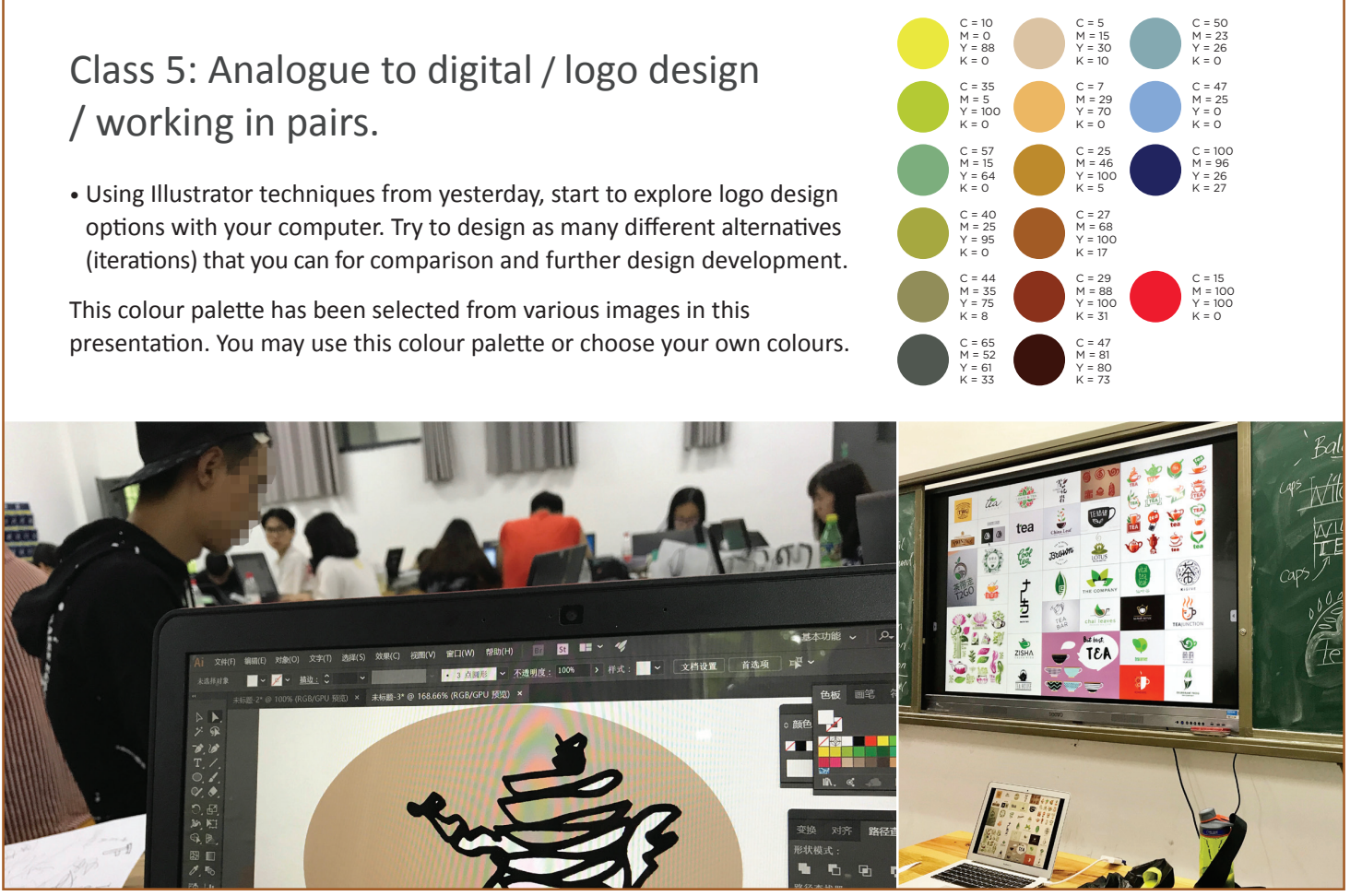

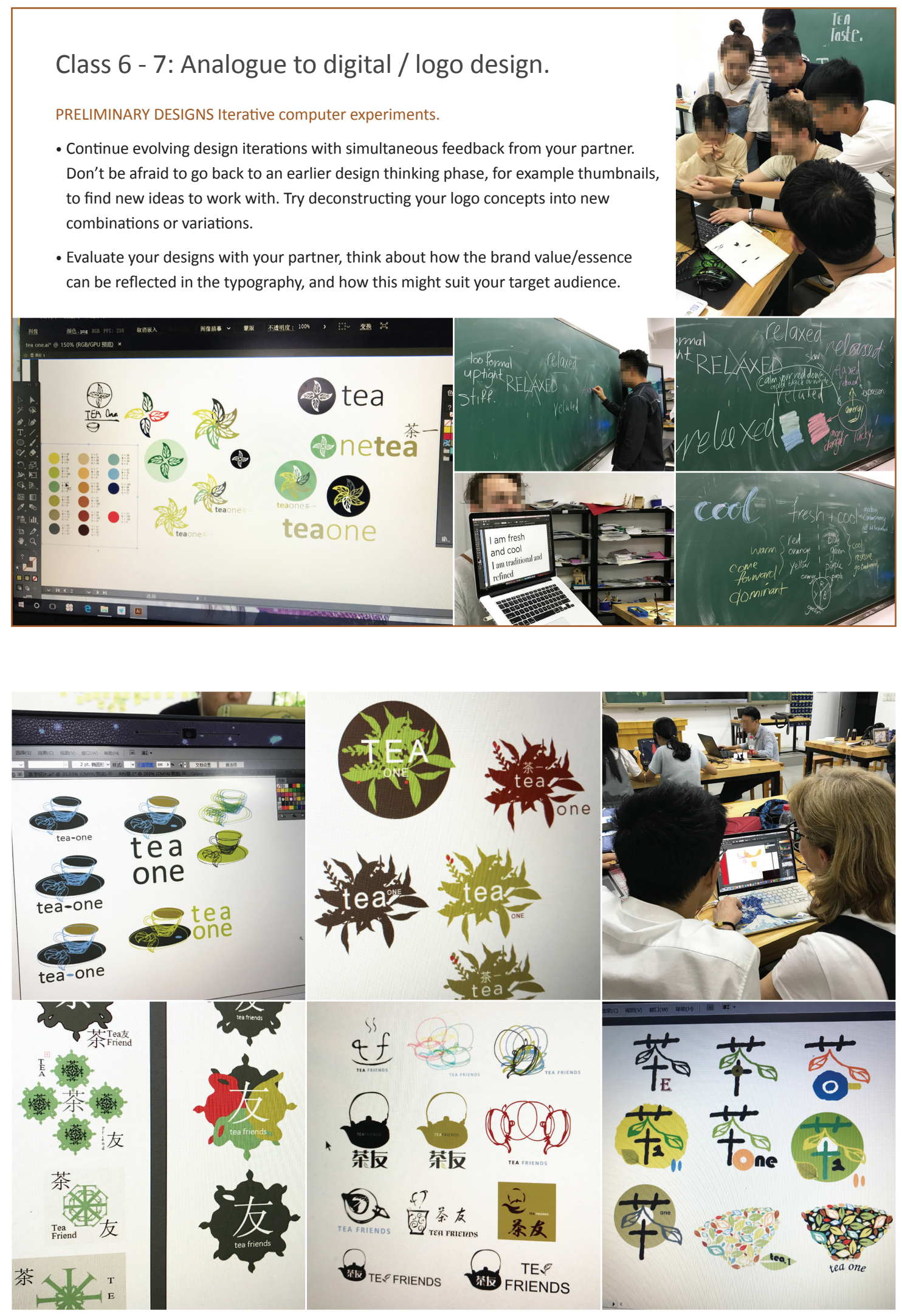
Class 8 - 10: Continued logo development and application of visual identity elements for branding.

In these classes your work evolves and develops into a number of visual identity elements which helps to define a 'look and feel' that relates to your chosen brand essence. The elements are applied in such a way, that all of the pieces feel like part of a family, but no piece is completely alike.

- Using the worksheets, deconstruct your logo designs into parts. Play and experiment with the Illustrator software techniques and the colour palette to re-assign colours to create a multitude of different pattern iterations.
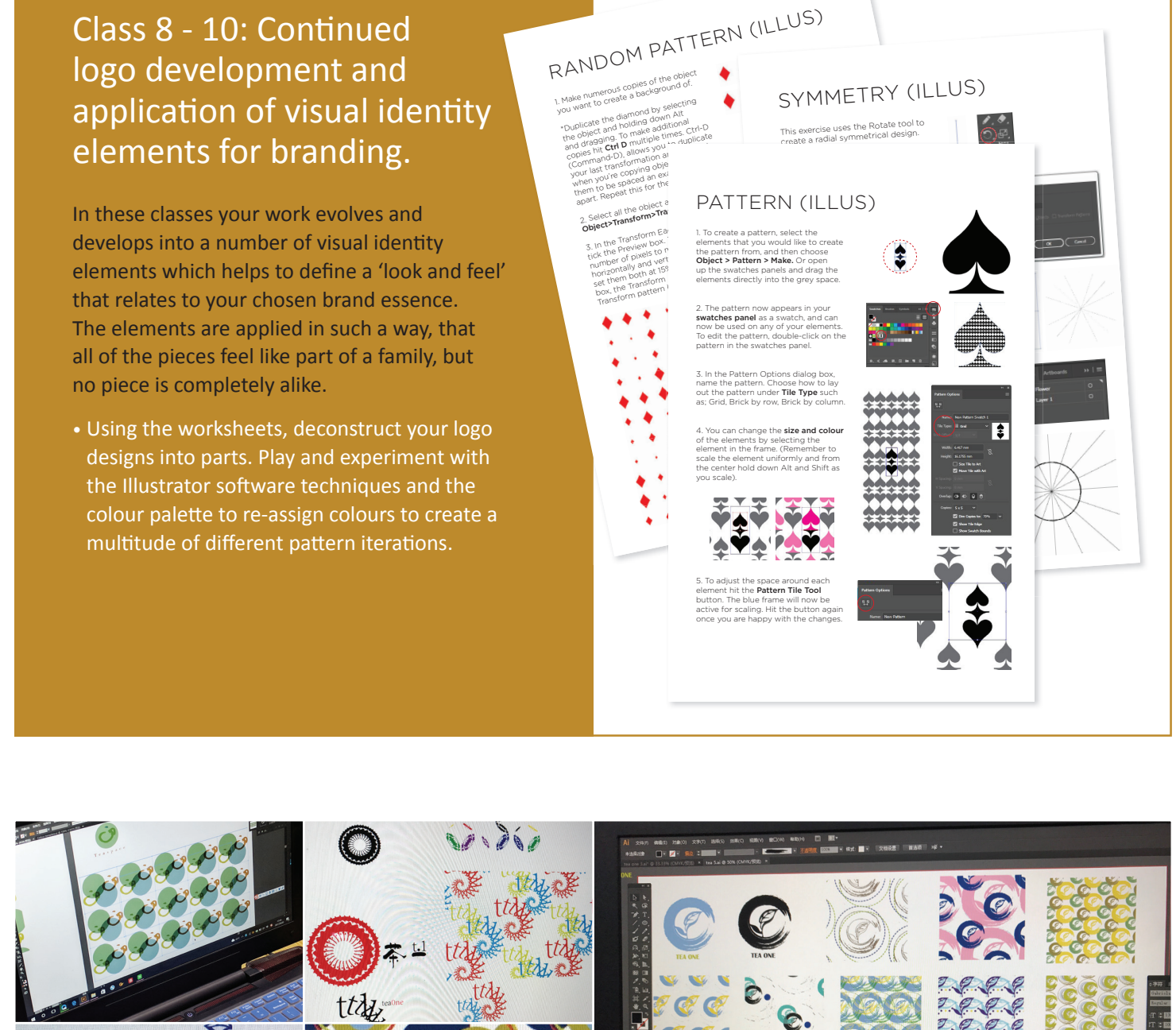

- $\div: 9: 90$.
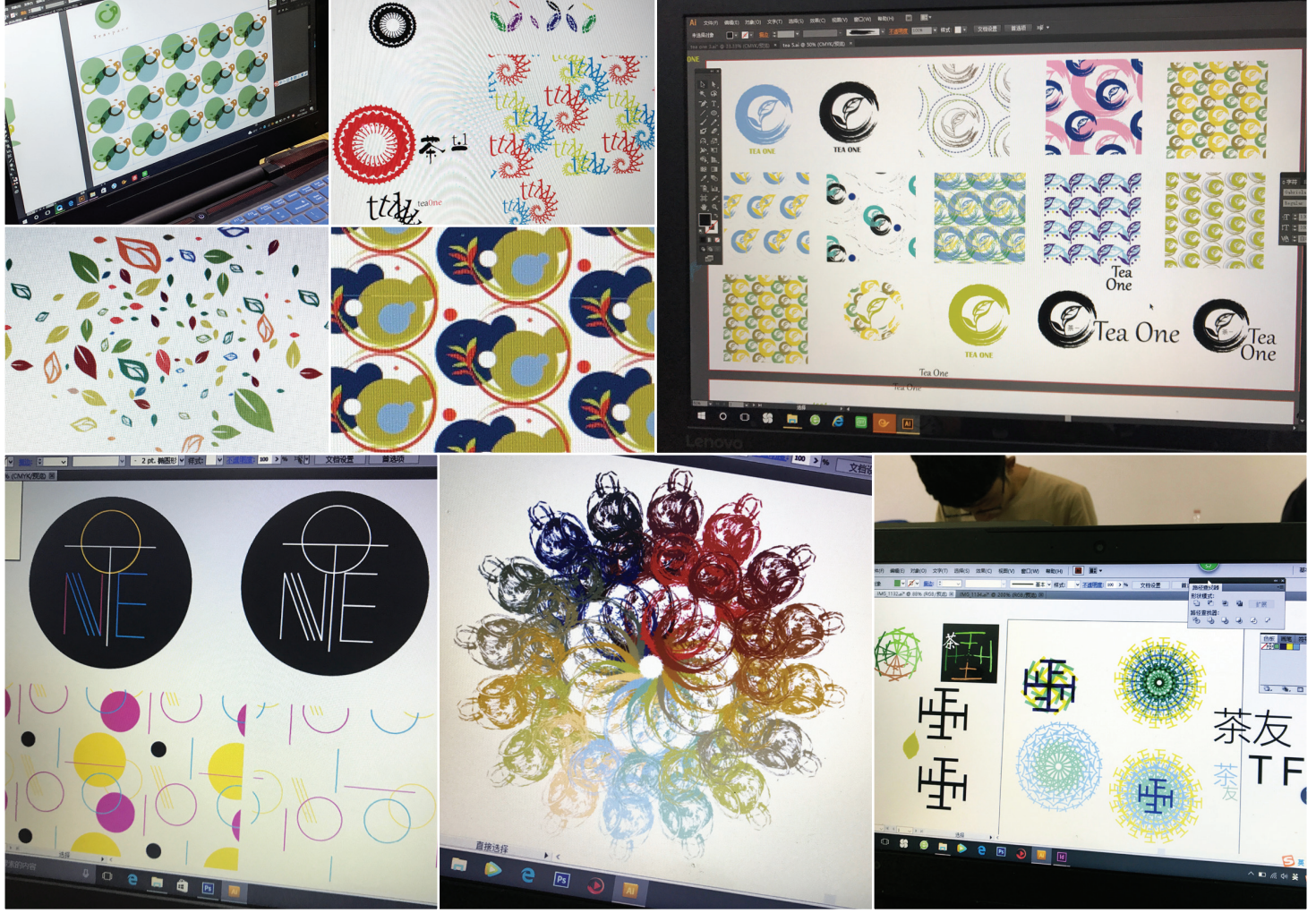


\section{Class 11 - 12: Development} and application / A3 Poster.

- Using the Photoshop (PS) template, apply your visual identity elements to the various tea house components. This is easily done by dragging vector ILLUS files into linked folders in the PS psd file. The vector work will convert to a smart object in PS (pixels).

- Using the A3 poster template, lay out your designwork ready for print on high quality paper (not gloss).

- Prepare a 150 word written reflection in English, including highlights of the project, successes and struggles. This is when you evaluate your work by getting feedback from others as well as your own self-evaluation. You'll want to think about changes, improvements and developments for your future design projects of this nature.
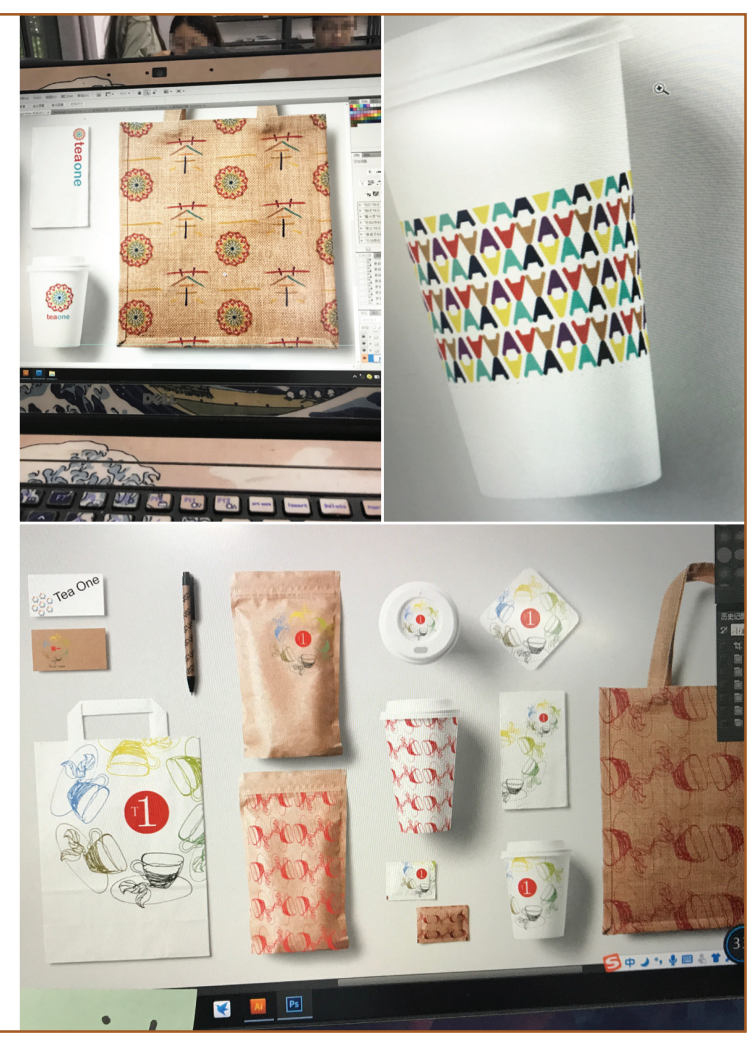

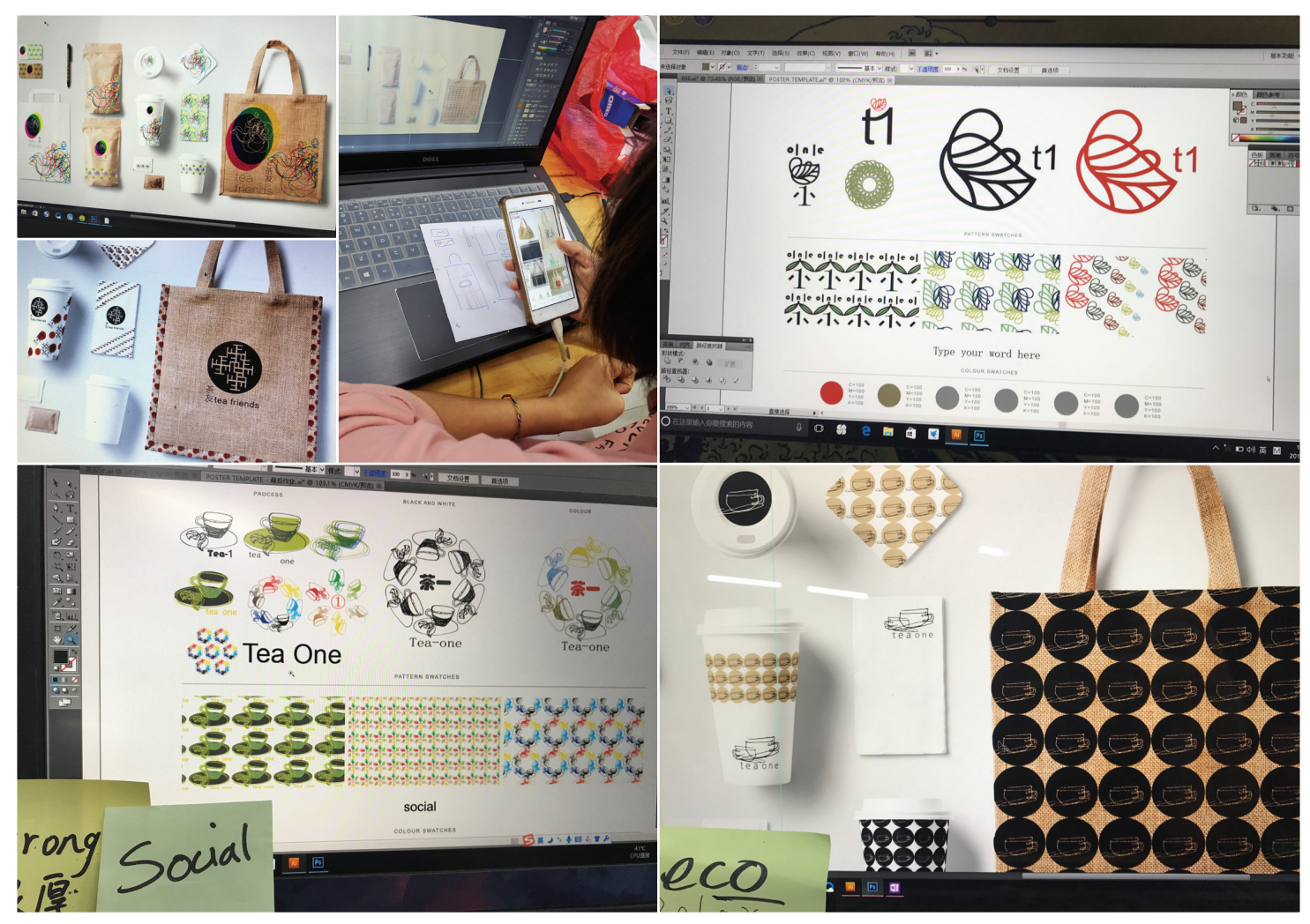




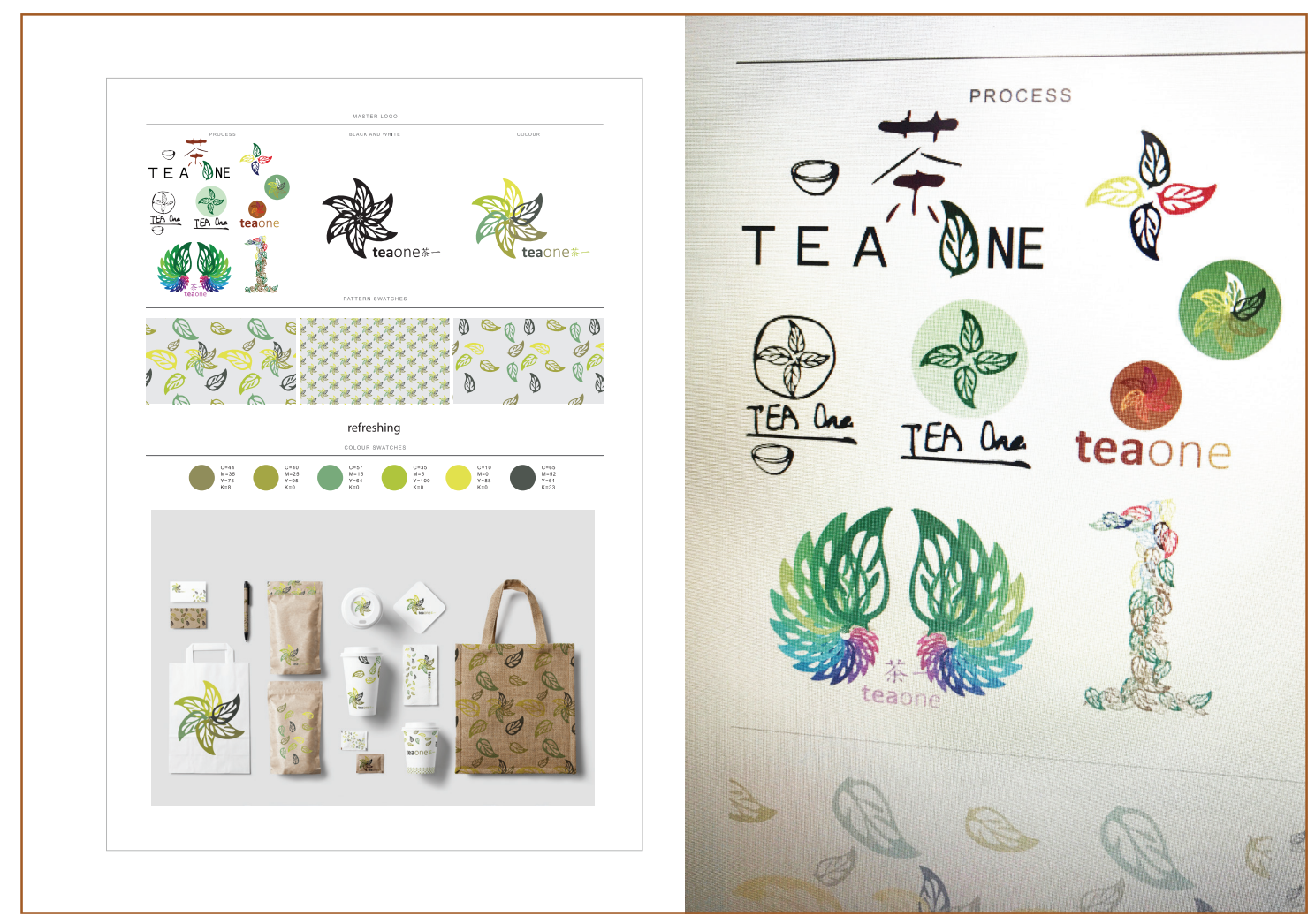

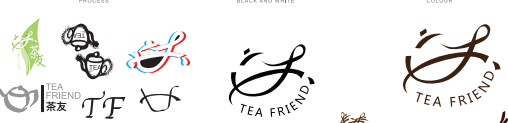

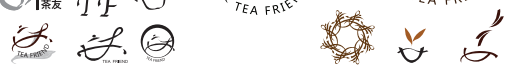

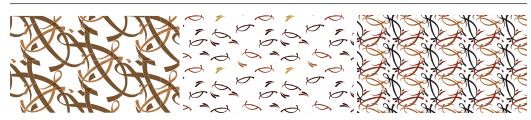
0

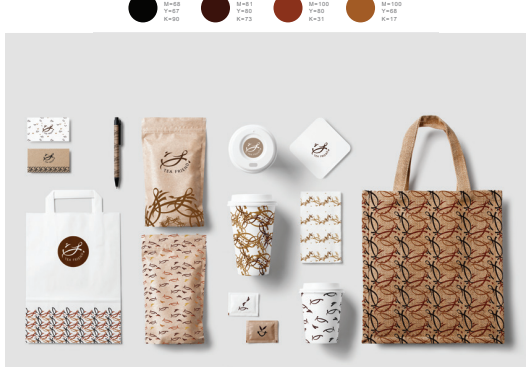

○菠

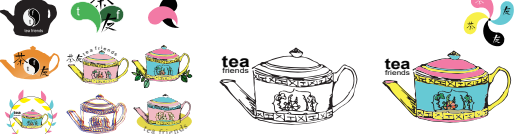

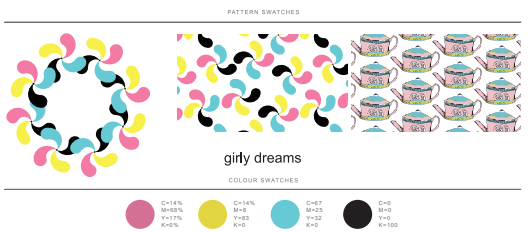

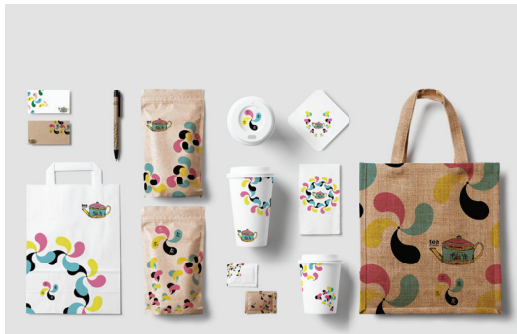


Appendix 7.1. Miao illustration project: Playing cards

\begin{tabular}{|c|c|c|c|}
\hline $\begin{array}{l}\text { Class } \\
\text { and date } \\
(2018)\end{array}$ & Design thinking phase & $\begin{array}{l}\text { Activity modes: } \mathrm{T}=\text { Thinking, } \\
\mathrm{E}=\text { Examining, } \mathrm{N}=\text { Notes, } \\
\mathrm{S}=\text { Sketching, } \mathrm{C}=\text { Computer }\end{array}$ & $\begin{array}{l}\text { Data gathering / } \\
\text { video capture } \\
\text { periods }\end{array}$ \\
\hline $\begin{array}{l}\text { 1. Monday } \\
21 \text { May }\end{array}$ & $\begin{array}{l}\text { Empathise/define. Problem } \\
\text { formation, concept creation } \\
\text { - Problem structuring }\end{array}$ & $\begin{array}{l}\text { T, E } \\
\text { Dyad formation } \\
\text { Moodboard. Visual reference }\end{array}$ & \\
\hline $\begin{array}{l}\text { 2. Tuesday } \\
22 \text { May }\end{array}$ & $\begin{array}{l}\text { Ideate. } \\
\text { - Idea generating process } \\
\text { Introduce zoomorphic, } \\
\text { anthropomorphic, } \\
\text { phyllomorphic and geometric } \\
\text { concepts }\end{array}$ & $\begin{array}{l}\text { T, E, N, } \mathbf{S} \\
\text { Dyad activity } \\
\text { 1. Thumbnail sketches to } \\
\text { interpret character for playing } \\
\text { card design } \\
\text { 2. Manual techniques }\end{array}$ & \\
\hline $\begin{array}{l}\text { 3. Wednesday } \\
23 \text { May }\end{array}$ & $\begin{array}{l}\text { Ideate. } \\
\text { - Idea generating process }\end{array}$ & $\begin{array}{l}\mathbf{T}, \mathbf{E}, \mathbf{N}, \mathbf{S} \\
\text { Dyad activity }\end{array}$ & \\
\hline $\begin{array}{l}\text { 4. Thursday } \\
24 \text { May }\end{array}$ & Technical, digital design & $\begin{array}{l}\text { C } \\
\text { Individual activity }\end{array}$ & \\
\hline $\begin{array}{l}\text { 5. Monday } \\
28 \text { May }\end{array}$ & $\begin{array}{l}\text { Ideate / prototype } \\
\text { Digital design } \\
\text { - Preliminary computer work }\end{array}$ & $\begin{array}{l}\text { T, E, S, C } \\
\text { Dyad and individual activity }\end{array}$ & \\
\hline $\begin{array}{l}\text { 6. Tuesday } \\
29 \text { May }\end{array}$ & $\begin{array}{l}\text { Ideate / prototype } \\
\text { Solution generation / colour } \\
\text { - Preliminary computer work }\end{array}$ & $\begin{array}{l}\text { T, E, } \mathbf{S} \\
\text { Dyad and individual activity }\end{array}$ & Recruitment \\
\hline $\begin{array}{l}\text { 7. Wednesday } \\
30 \text { May }\end{array}$ & $\begin{array}{l}\text { Ideate / prototype } \\
\text { Evolution and evaluation } \\
\text { - Iterative process }\end{array}$ & $\begin{array}{l}\mathbf{T}, \mathbf{E}, \mathbf{S}, \mathbf{C} \\
\text { Dyad activity }\end{array}$ & $\begin{array}{l}\text { Deconstruct and } \\
\text { reconstruct suits } \\
\text { for new variations } \\
\text { ( } 2 \text { hours) }\end{array}$ \\
\hline $\begin{array}{l}\text { 8. Thursday } \\
31 \text { May }\end{array}$ & $\begin{array}{l}\text { Ideate / prototype } \\
\text { Evolution and evaluation } \\
\text { - Iterative process }\end{array}$ & $\begin{array}{l}\mathbf{T}, \mathbf{E}, \mathbf{S}, \mathbf{C} \\
\text { Dyad activity }\end{array}$ & $\begin{array}{l}\text { Combine suit and } \\
\text { character design } \\
\text { ( } 2 \text { hours) }\end{array}$ \\
\hline $\begin{array}{l}\text { 9. Monday } \\
4 \text { June }\end{array}$ & $\begin{array}{l}\text { Ideate } \\
\text { Symmetry • Iterative process }\end{array}$ & $\begin{array}{l}\text { T, E, C } \\
\text { Dyad and individual activity }\end{array}$ & \\
\hline $\begin{array}{l}\text { 10. Tuesday } \\
5 \text { June }\end{array}$ & $\begin{array}{l}\text { Ideate } \\
\text { Pattern • Iterative process }\end{array}$ & $\begin{array}{l}\text { T, E, C } \\
\text { Dyad and individual activity }\end{array}$ & \\
\hline $\begin{array}{l}\text { l1. } \\
\text { Wed. } 6 \text { June }\end{array}$ & $\begin{array}{l}\text { Evaluate } \\
\text { Layout and review }\end{array}$ & $\begin{array}{l}\text { T, E, C } \\
\text { Dyad and individual activity }\end{array}$ & \\
\hline $\begin{array}{l}12 . \\
\text { Thurs. } 7 \text { June }\end{array}$ & $\begin{array}{l}\text { Evaluate } \\
\text { Review and hand-in }\end{array}$ & $\begin{array}{l}\text { T, E, C } \\
\text { Dyad and individual activity }\end{array}$ & \\
\hline
\end{tabular}

Appendix table 2. Overview of twelve Miao illustration project classes and video capture periods.

The following 'slides' are a summary of information and instruction conveyed to the students in the twelve classes, and photographic evidence of the learning. 
(Source: Unless indicated, all are author images of VCD student work at HNCU).

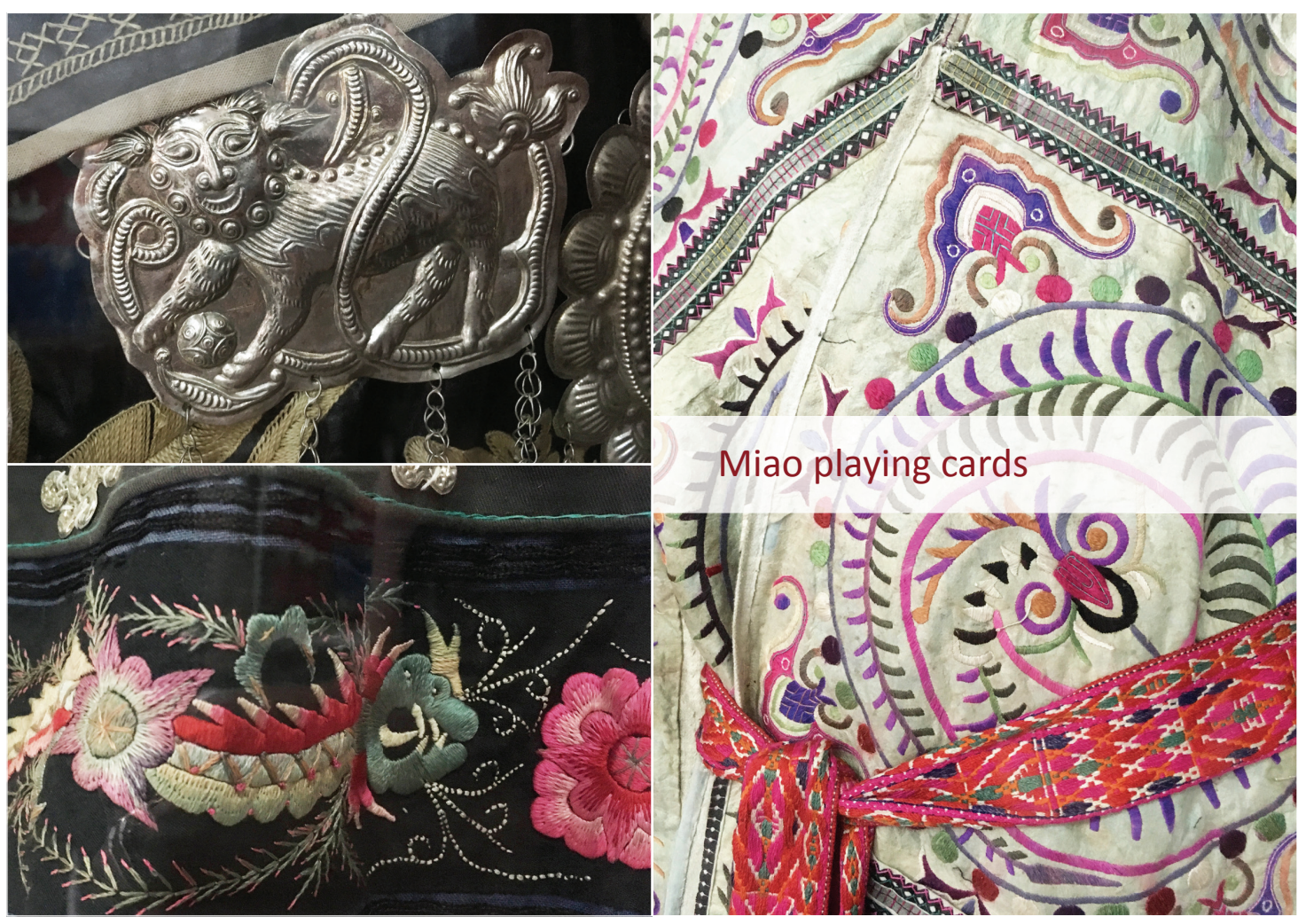

Source: Textiles at the Shanjiang Miao Museum, western Hunan (Xiangxi) (author images).

\section{Class 1: Project introduction and research}

1. The class will begin with an introduction to the project and examples of existing card designs will be shown.

2. Play a game of snap with the aim of assigning pairs with a card rank to work with for the duration of the paper, i.e. two of you will work together on four cards $(10 * 10 * 10 \vee 10 \$)$. The first to snap a pair should remove the two cards from the deck, write your name on the cards and watch the game continue. Once all the cards are snapped and distributed find your number (card rank) partner.

3. With your partner research and create a mood board for the playing card character you have won. A mood board is an arrangement of images related to a design theme, and is used as a reference point.

4. Next class, please bring: printed A3 mood board, drawing and tracing paper, pencils, pens, red and black marker pens and glue sticks.

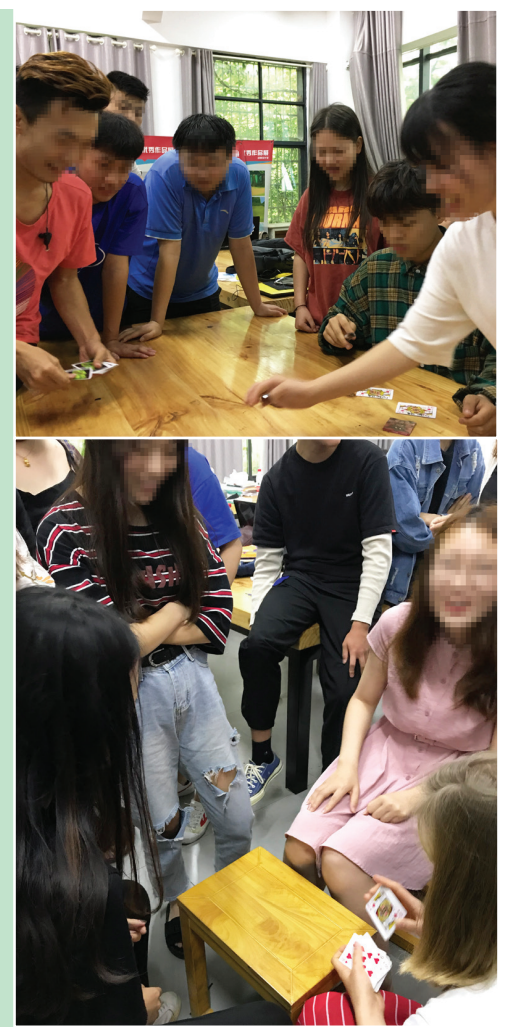



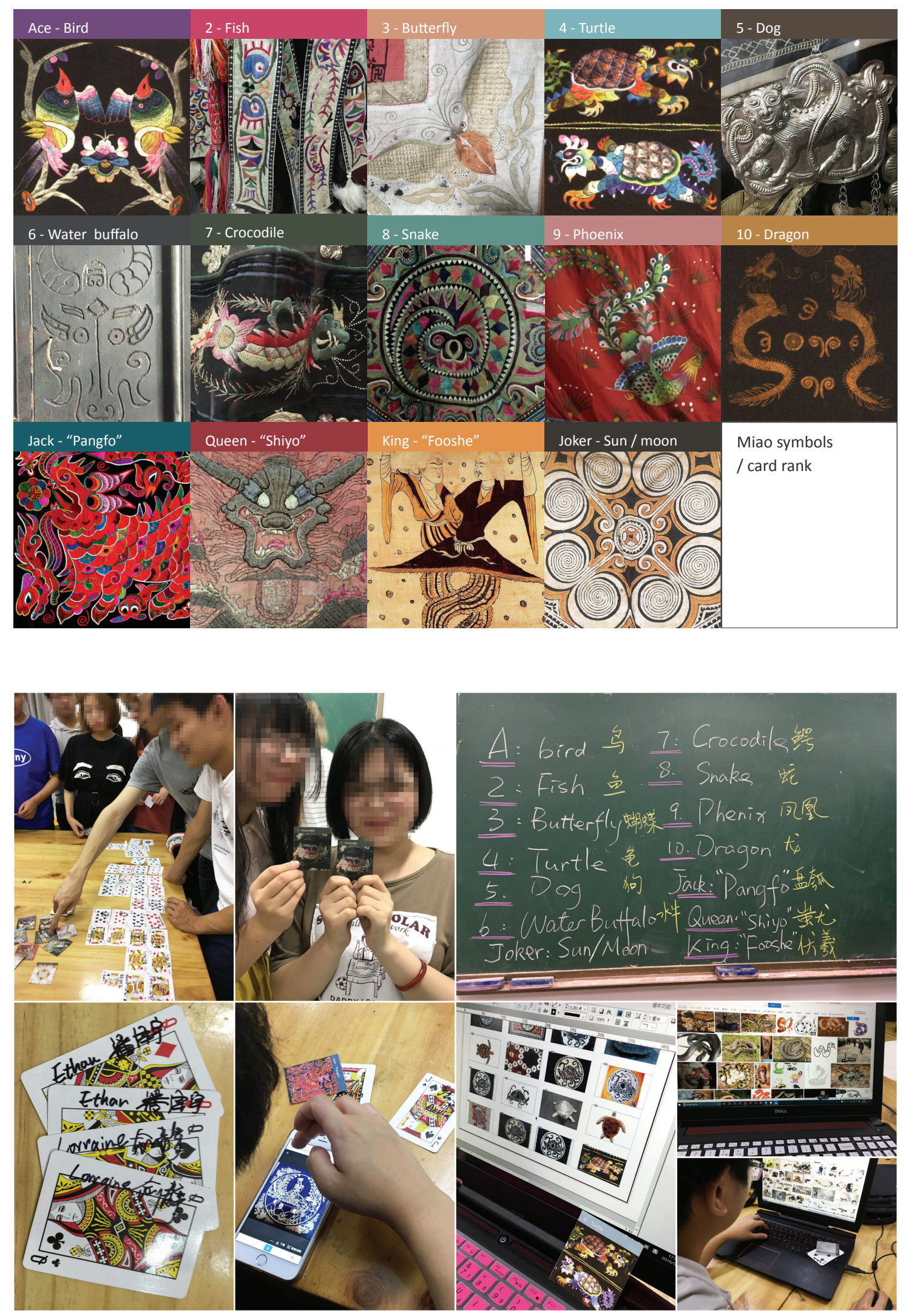


\section{Class 2: Idea generation}

1. Reinterpret your Miao symbol through drawn explorations. Aim to generate as many drawings as possible. Begin idea generation with your partner by brainstorming and making thumbnail sketches using your mood board as a reference.

2. Continue to develop your ideas by using a variety of techniques, such as; (re)-drawing, cutting, folding, overlapping, and tracing.

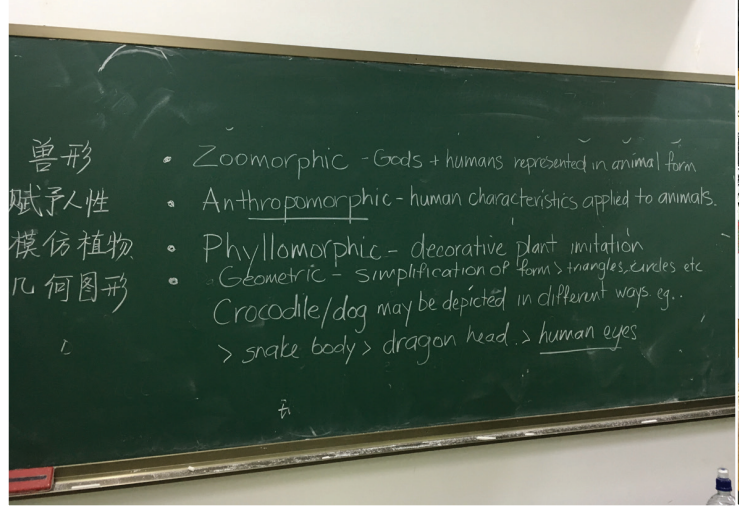

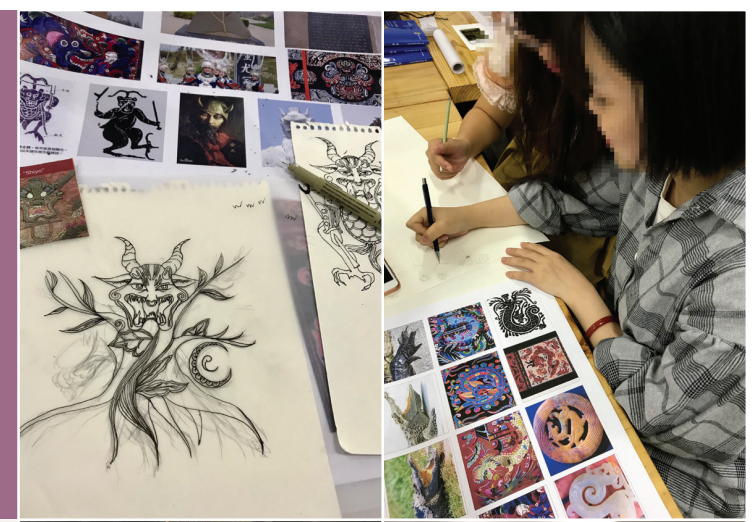

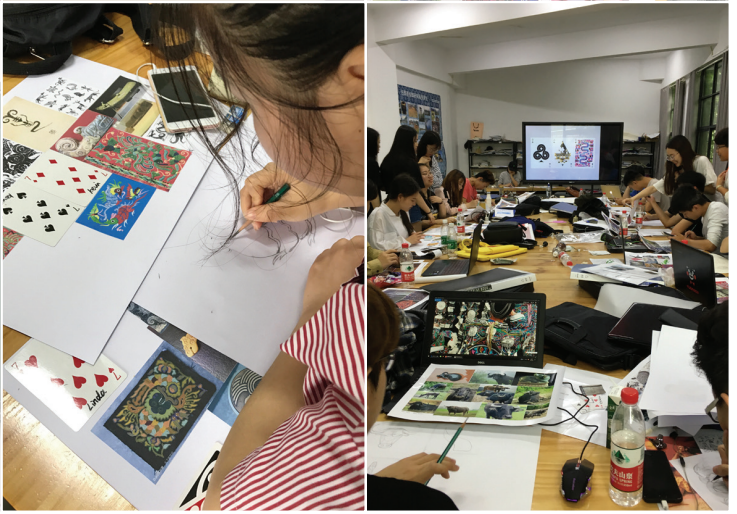

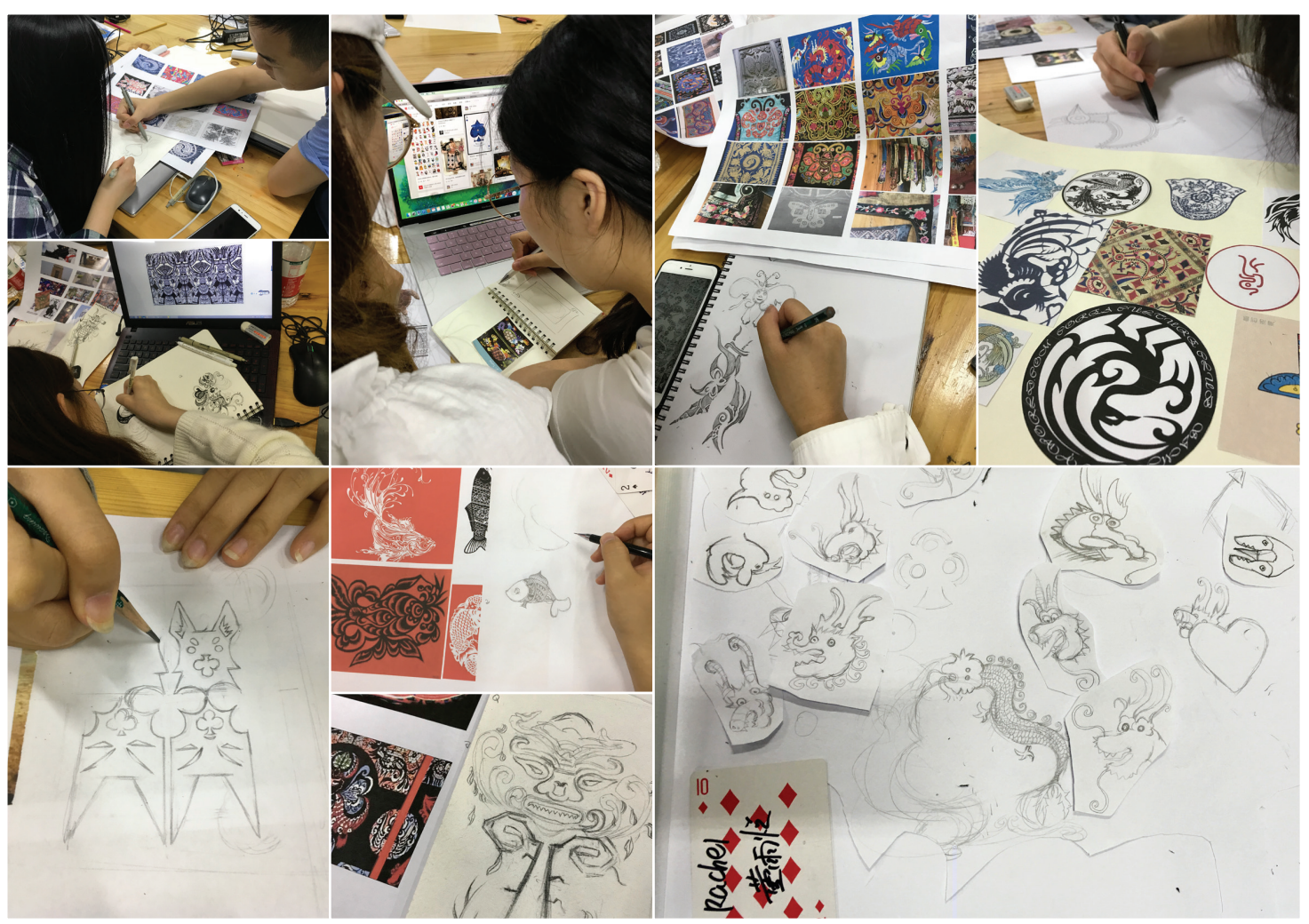



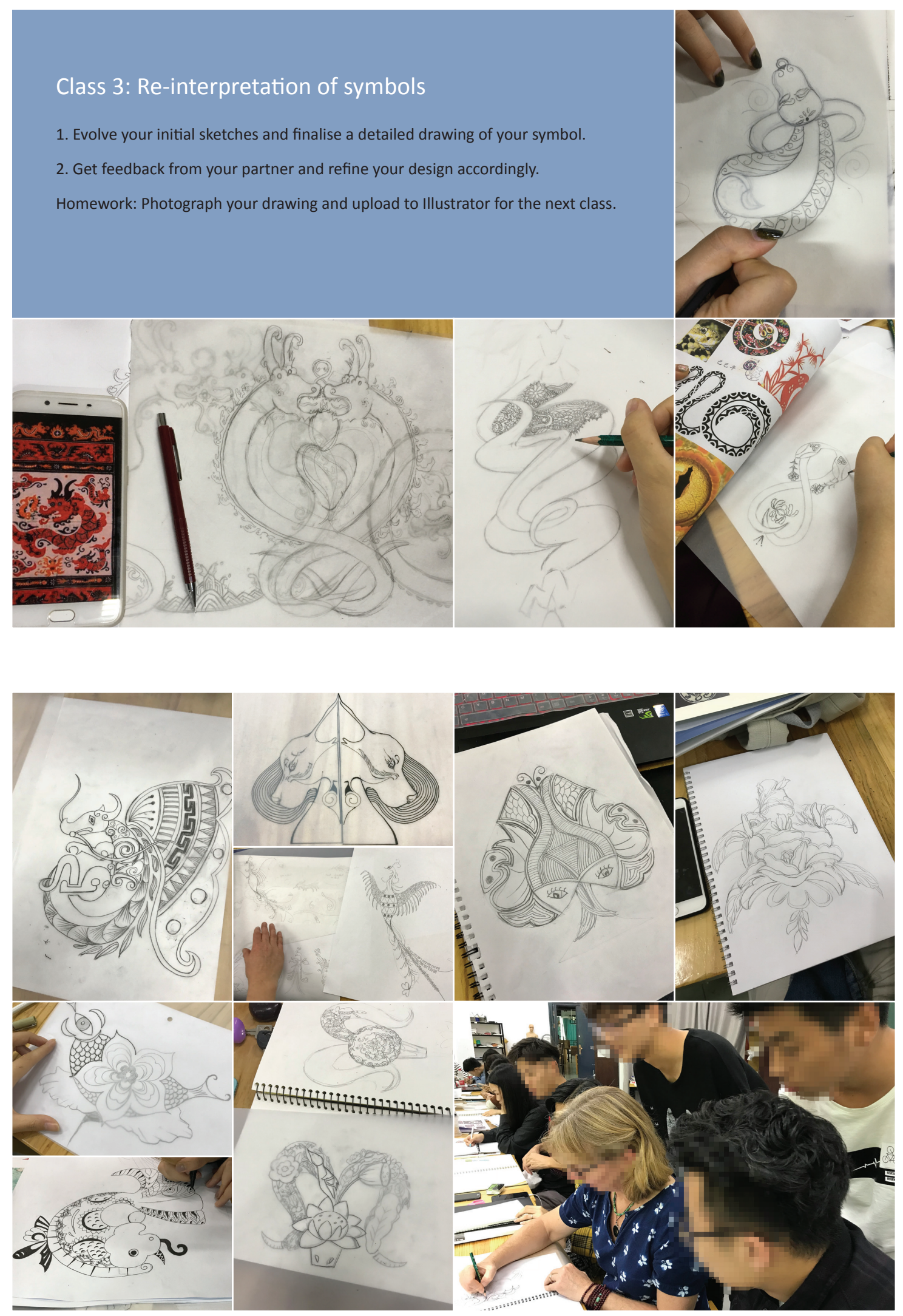

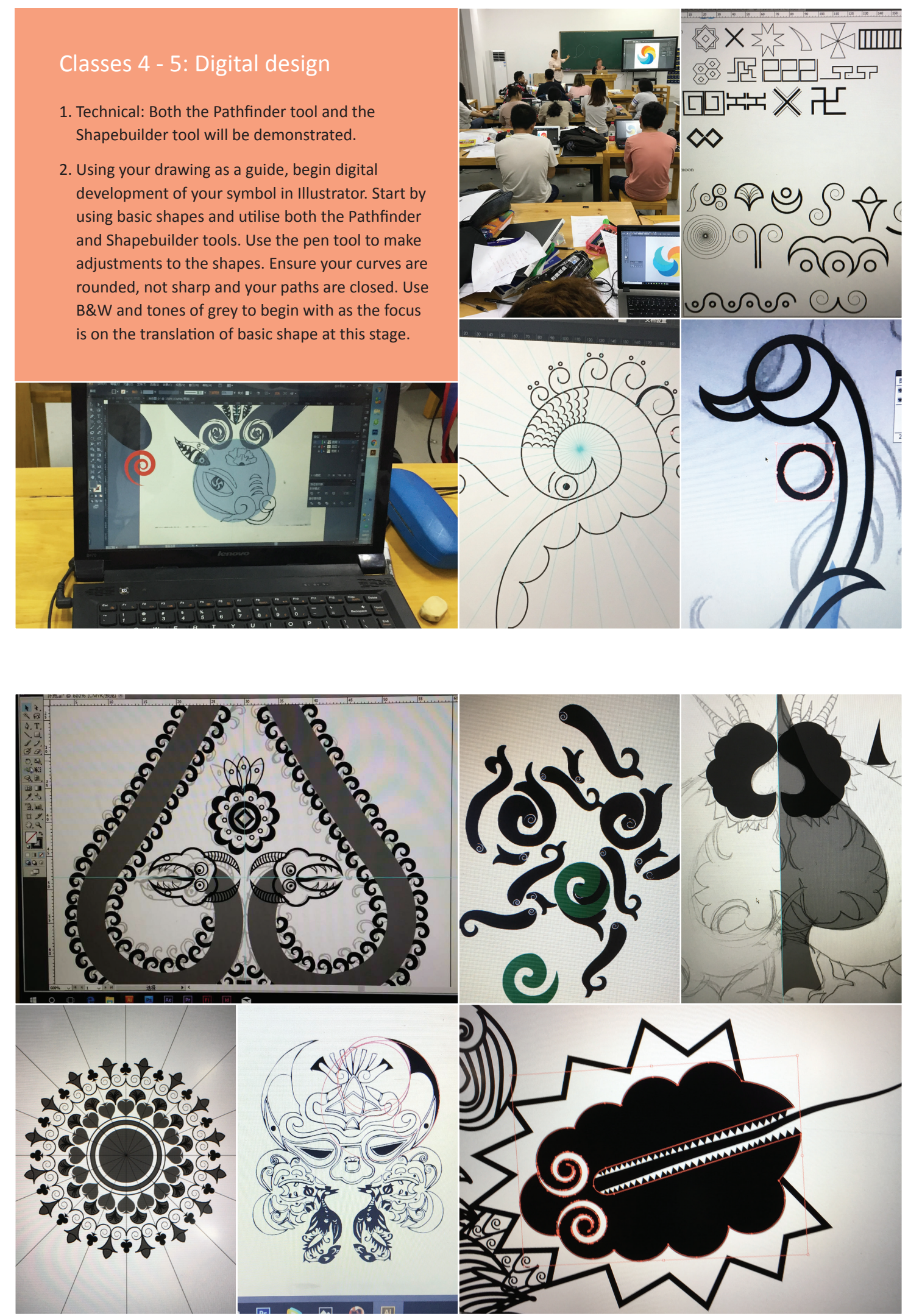


\section{Class 6: Colour}

1. Technical: The blend tool and custom brushes will be demonstrated. Consider ways to use these techniques in your design work.

2. Add colour to your design by using the swatches provided. Explore a number of different colour options for the designs on the cards.

Homework: Printout your design at A3 for your workbook and to pin up for a class critique.

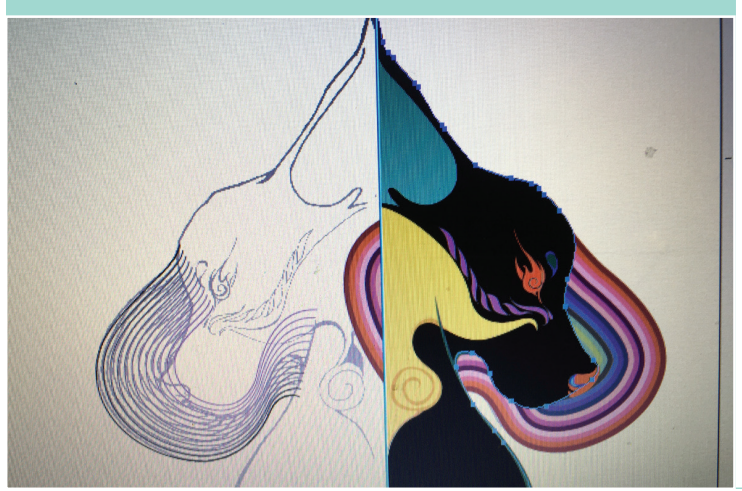

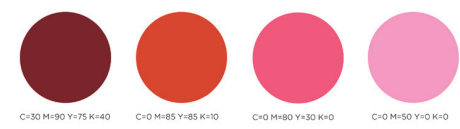
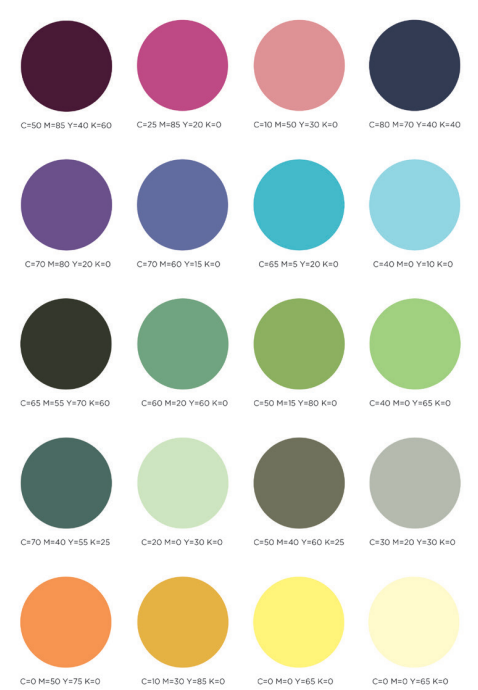
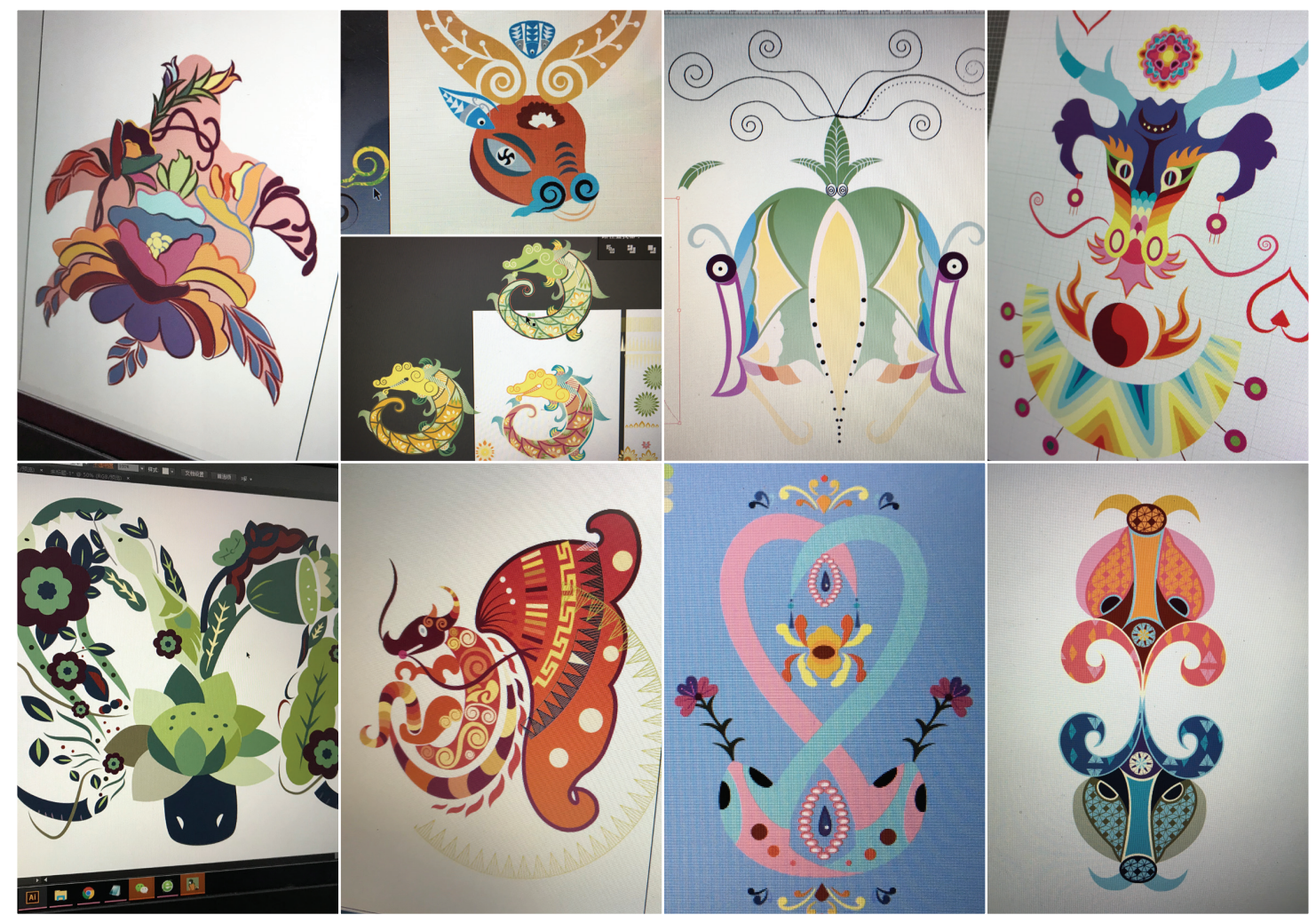
Class 7: Designing the suits

梅花, 方块, $\vee$ 红心, 黑桃

1. Preliminary designs: Create a number of design iterations for each suit (1 suit per 20 mins). With your partner, develop your concepts through drawing, cutting (red and black paper), overlapping, and tracing.

2. Digital development: With your partner choose one final concept for each suit and create a digital versions. Each individual should develop two of the suits. Your concept should be explored in multiple ways. The more you create the more you'll find new ideas and approaches are generated.
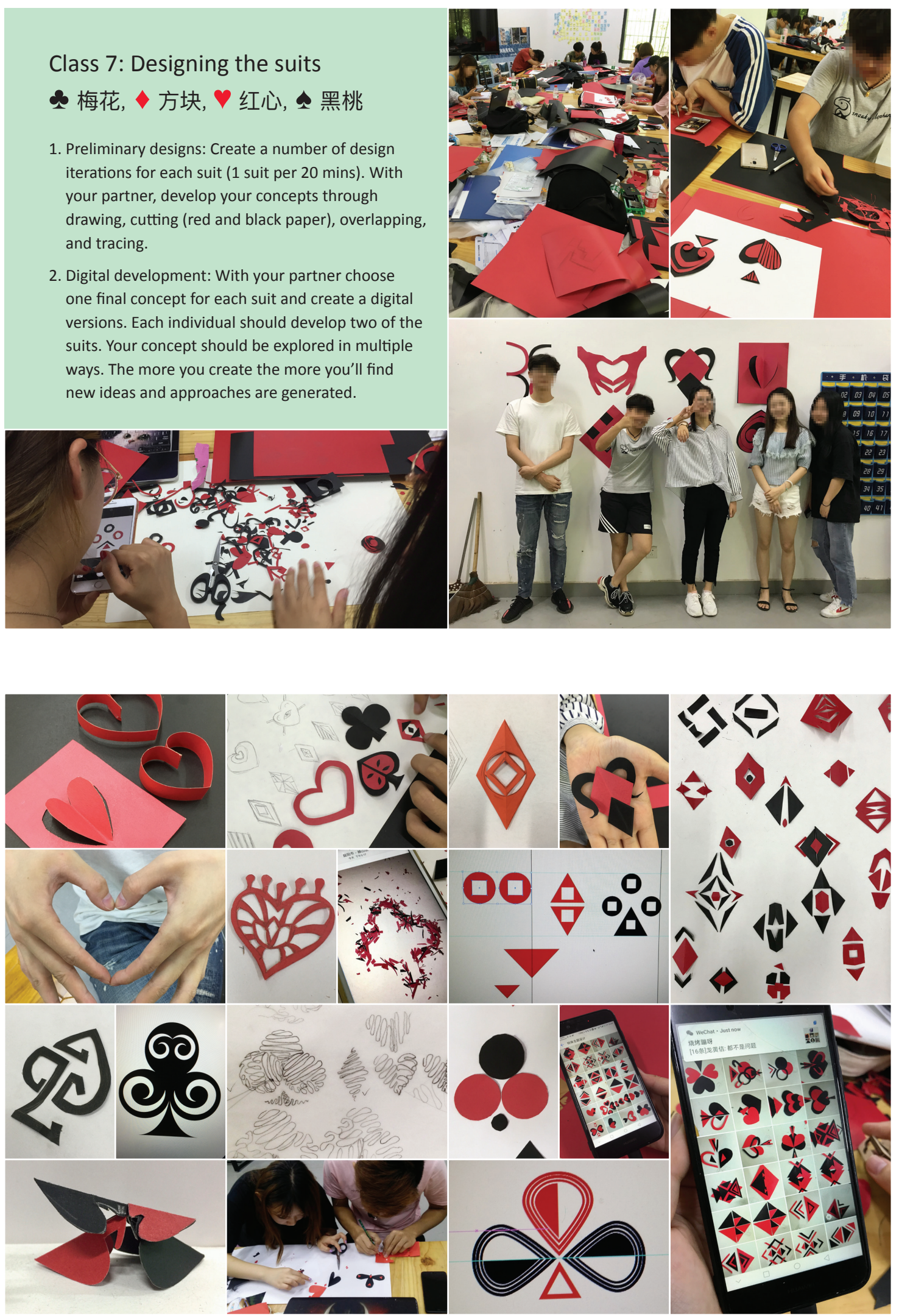

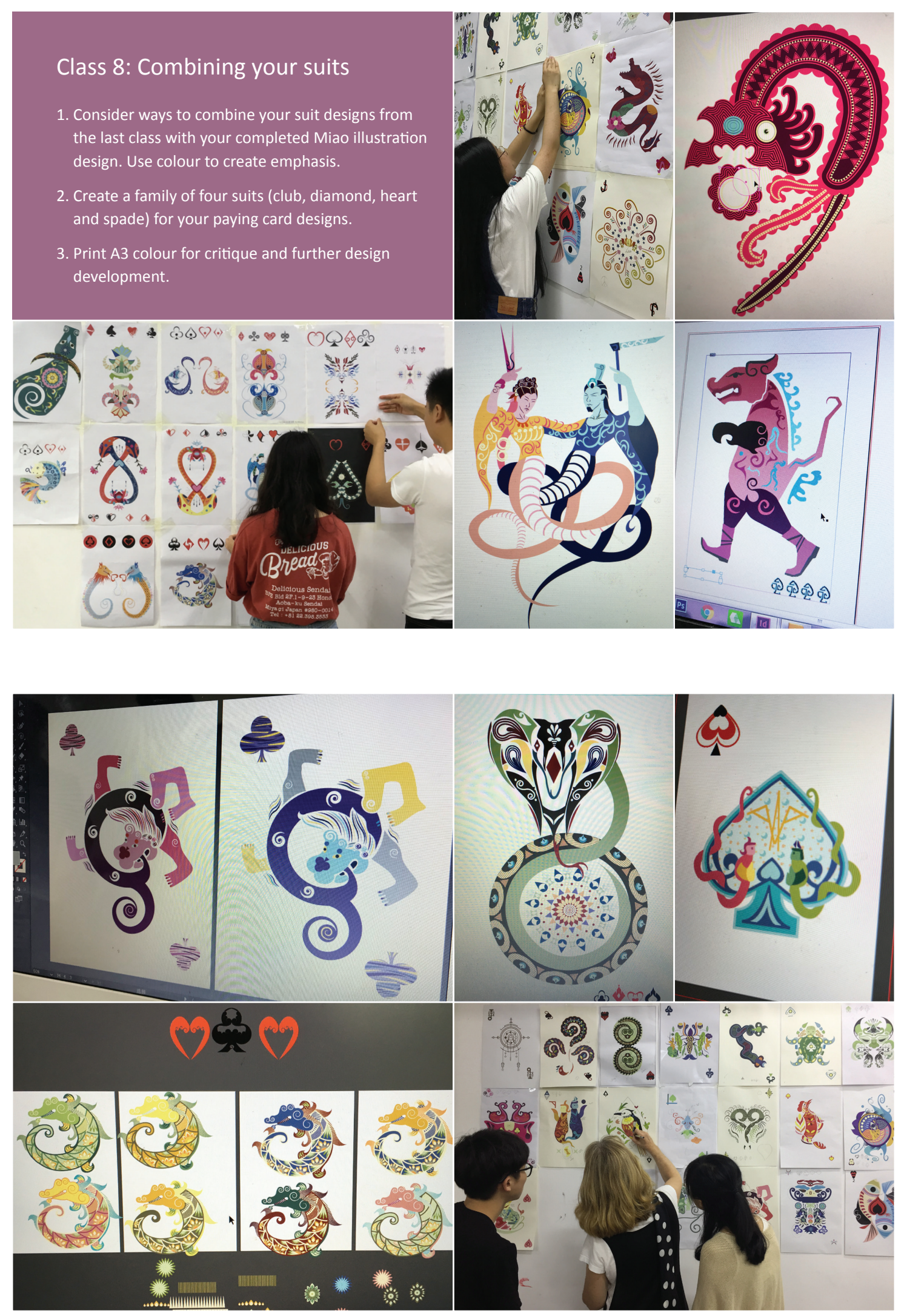


\section{Class 9: Symmetry}

Symmetry is where the elements on one side of the design balanced with those on the other side. Remember there are many types of symmetry: rotations, reflections, geometric, and radial.

1. Technical: The rotate tool will be demonstrated to create a radial symmetrical design.

2. Create a variety of symmetrical designs with your selected motif which depict your card rank (number). Additional elements may be used.
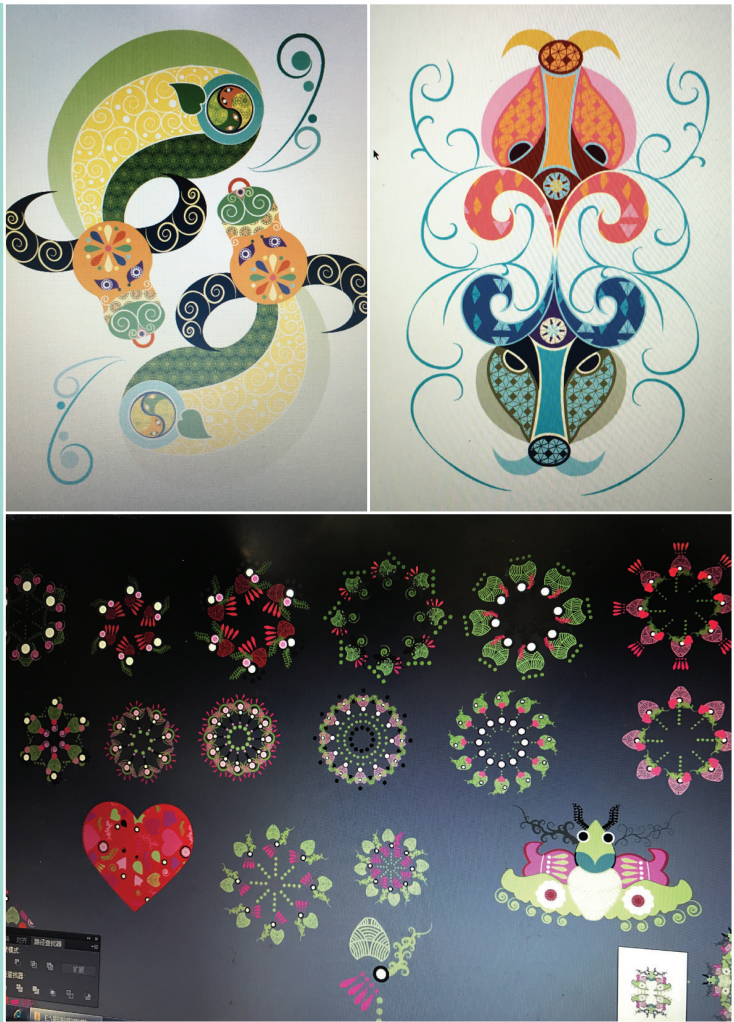

\section{Class 10: Pattern}

Pattern is the repeating of objects or symbols and has often become an integral part of cultures stylistic conventions.

1. Create a variety of pattern designs using elements from your final design. Additional elements may be used. These patterns will be used for the back of the playing cards.

2. Consider ways to create rhythm in the overall design and applying the patterns on a coloured background.

By the end of the class you should have completed at least 15 different patterns.
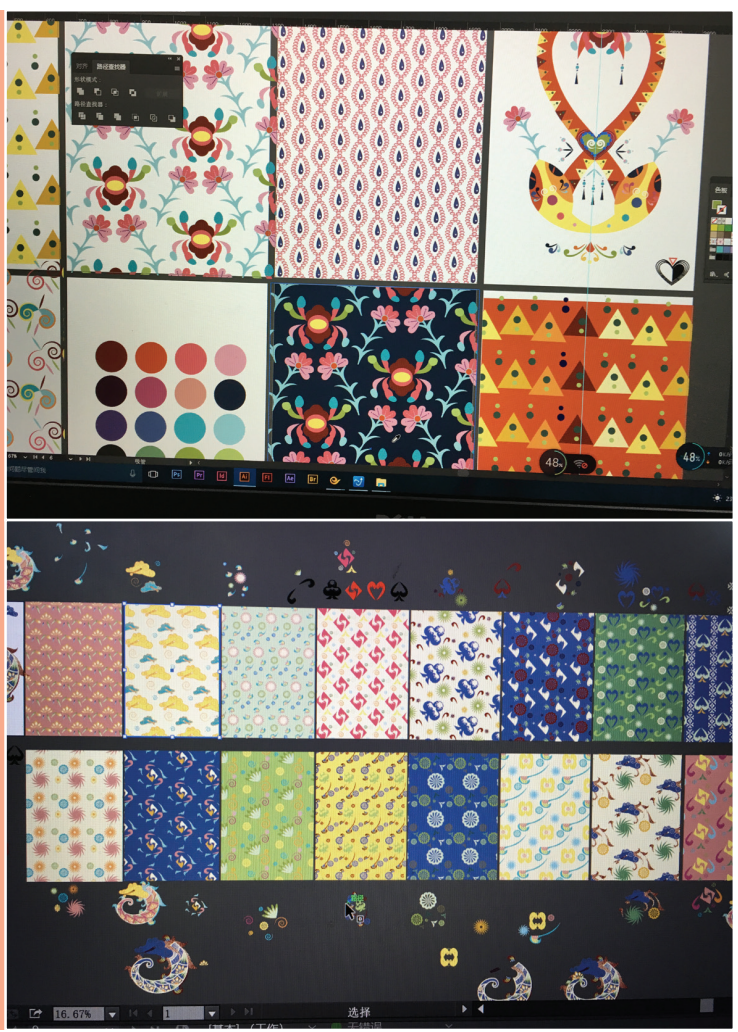


\section{Class 11 - 12: Layout and review}

This is when you evaluate your work by getting feedback from others, make any adjustments to your work and reflect on the design process.

Your task is to layout your final playing card design and patterns within the A4 INDD template. Consider alternate colour ways across the four cards.

See the next slide for hand-in details.

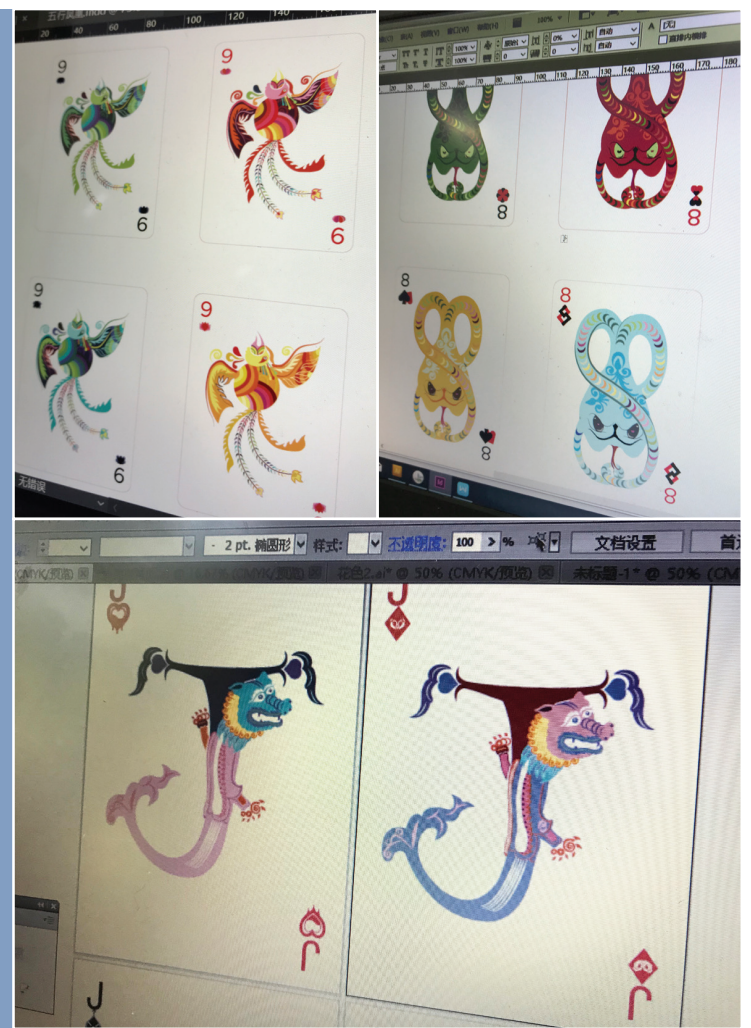

\section{Class 12: Hand-in}

1. Marking poster - A3 Poster using the INDD template. Include your name, student ID, course name: Miao special topic, teachers name and score on the bottom right hand corner, 10pt Arial.

2. Playing cards - Two A4 double sided printouts. Print four cards on one side and one pattern on the other side. The pattern should cover the entire page to allow for printer trimming. 200 gsm card stock, cmyk.

3. Exhibition poster - A2 poster of your final design. Include your four suit designs on the bottom right. Set the margins to $50 \mathrm{~mm}$ and write your name on the bottom left hand corner, 18pt Arial.

4. Workbook - Clearly named with a photograph on the front cover. Include mood boards, drawings and printouts of design developments.

5. Reflection - Prepare a 150 word written reflection in English which answers the following questions: How did you arrive at your final design (solution)? What was difficult for you? What helped you?

6. Ai file - Monitor to collect your final Illustrator file.
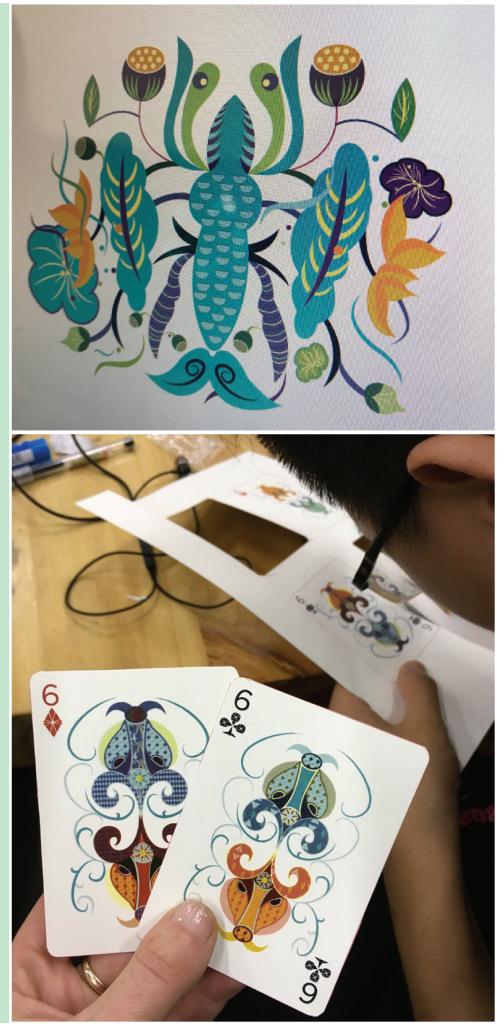

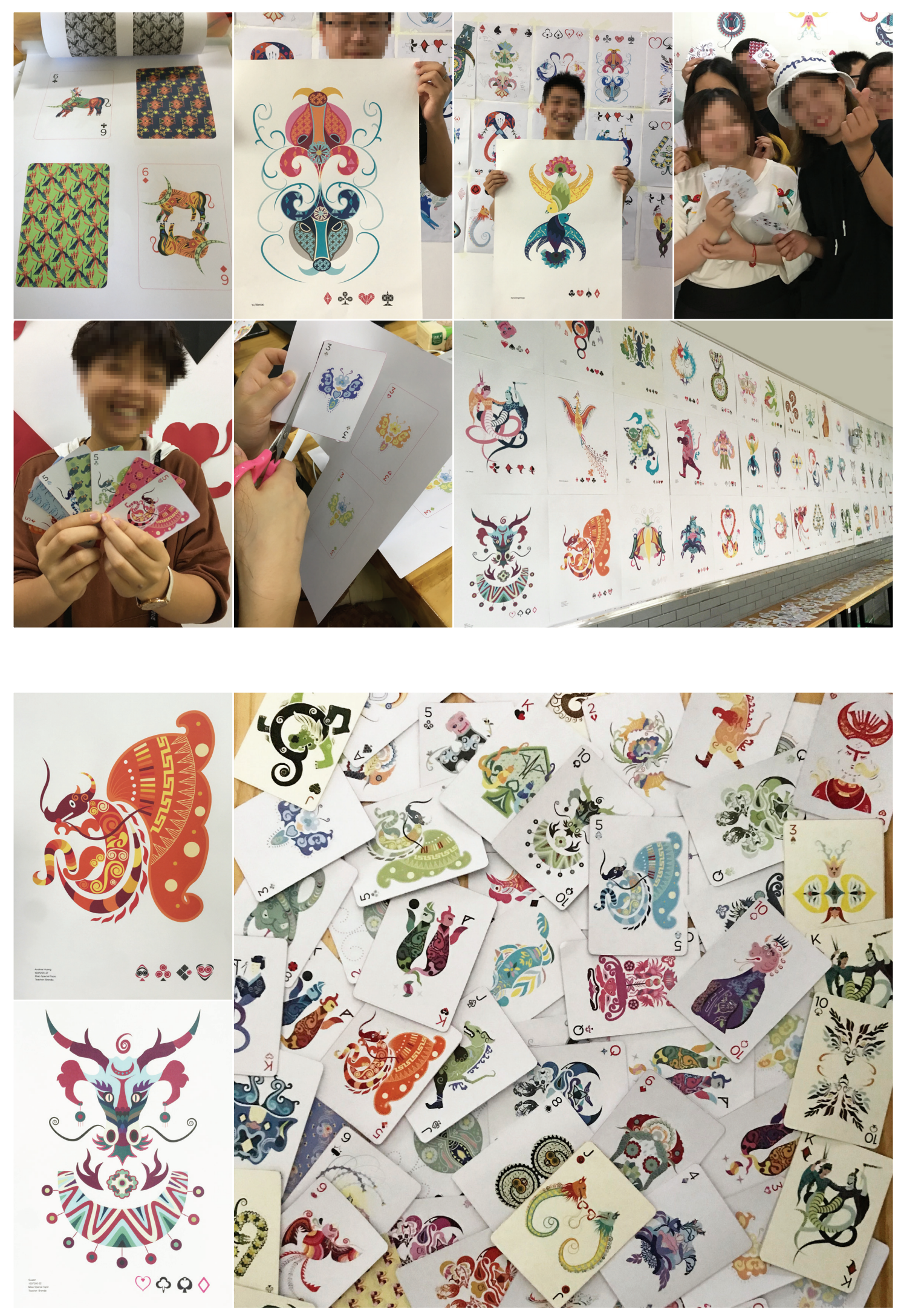